Measure functional differential equations and impulsive functional dynamic equations on time scales

Jaqueline Godoy Mesquita 


\title{
Measure functional differential equations and impulsive functional dynamic equations on time scales
}

\author{
Jaqueline Godoy Mesquita
}

Advisor: Prof. Dra. Márcia Cristina Anderson Braz Federson

Co-advisor: Prof. Dr. Antonín Slavík

Doctoral dissertation submitted to the Instituto de Ciências Matemáticas e de Computação - ICMC-USP, in partial fulfillment of the requirements for the degree of the Doctorate Program in Mathematics. FINAL VERSION. 
Ficha catalográfica elaborada pela Biblioteca Prof. Achille Bassi e Seção Técnica de Informática, ICMC/USP, com os dados fornecidos pelo(a) autor(a)

Godoy Mesquita, Jaqueline

Measure functional differential equations and impulsive functional dynamic equations on time scales / Jaqueline Godoy Mesquita; orientadora Márcia Cristina Anderson Braz Federson; coorientador Antonín Slavík. -- São Carlos, 2012. $214 \mathrm{p}$.

Tese (Doutorado - Programa de Pós-Graduação em Matemática) -- Instituto de Ciências Matemáticas e de Computação, Universidade de São Paulo, 2012.

1. Measure equations. 2. Functional differential equations. 3. Impulsive equations. 4. Time scales. 5. Generalized ODEs. I. Anderson Braz Federson, Márcia Cristina, orient. II. Slavík, Antonín, coorient. III. Título. 
"There is no branch of mathematics, however abstract, which may not some day be applied to phenomena of the real world".

(Nikolai Lobachevsky)

"Gosto de ser gente porque, inacabado, sei que sou um ser condicionado mas, consciente do inacabamento, sei que posso ir além dele".

(Paulo Freire) 


\section{Acknowledgment}

First of all, I would like to thank Sri Sathya Sai Baba for everything in my life and for giving me all the necessary force to continue my journey.

I am really grateful to Luís Góes Mesquita, my husband, who always believed in me and gave me all the necessary support to deal with the difficulties in my life, encouraging me in all the moments. He is the most responsible for all my professional success. Without him, I would not arrive until here. His trust, love, company, friendship, encouragement and fellowship were fundamental to me during this period. I do not have words to express his meaning in my life. Luís, thank you for everything, specially for being an essential and fundamental part of my life. I love you very much!

I am grateful to my dear parents. In special, to my mother, Maria Aparecida Bezerra, for everything. She was my pillar during all these years, maintaining me strong to overcome all the difficulties of my life. Without her support, I would not be here. Also, I am grateful to my dad, Gilson Godoy, for all his trust, encouragement, patience and love. He gave me the necessary support to arrive until here, listening me during hours, advising me always. I do not have words to express the importance of my parents to my life and how proud and glad I am to be their daughter. Thank you for everything!

I am grateful to my sister Cynthia for being one of my best friends and an essential part of my life. Also, I am grateful to my brothers, Victor, Louise and Vinicius, for their affection and friendship.

I am grateful to my second parents: Elaine and Márcio, my grandmother Maria Tereza, my aunts Cleidinha and Patrícia and, also, Marcos Malta for all their love and trust.

I am grateful to my parents-in-law, Ana Maria Medeiros Góes and Alcione Mesquita, and to my brothers-in-law, Paulo Mesquita and Pedro Mesquita, for being so special and 
caring with me. Their affection and trust were fundamental to bring me till here.

I would like to thank my dear friends: Pedro Lima, Renato Alejandro, Ismael Sobrinho, Laura Lobato, Roberta Pacheco, Ton Fortes, Paulo Mendes, Suzete Afonso, Lívia Gomes, Vinicíus Facó, Patrícia Tacuri, Matheus Bortolan, Luana Mesquita and Eduard Toon. Without their support, I would not be here. All of them shared with me a lot of unforgettable moments. I am really glad to have all of them in my life.

I would like to thank my advisor, professor Márcia Federson, for all the opportunities that she provided me during my $\mathrm{PhD}$ course, encouraging me to improve my career as a researcher, showing a lot of opportunities. Under her supervision and knowledge, I was able to improve myself as a mathematician, developing several works in collaboration with reputed researchers. I am really grateful to her for this and for her friendship.

I am grateful to my co-advisor, professor Antonín Slavík, who gave me all the necessary support during my stay in Prague. He taught me a lot and shared with me his knowledge. This was really important to me. Also, he shared with me a lot of good moments in Prague. I appreciated a lot his company during our trips and meals. I am really grateful for his friendship, patience and trust.

I would like to thank professor Milan Tvrdý, who was part of my family in Prague. Without him, I would not be able to finish my sandwich doctorate. He helped me a lot with my visa, and I do not have words to describe how I am grateful to him. Also, I am grateful for his company, patience and advises, which were really important to me.

I would like to thank professor Maria do Carmo Carbinatto for her friendship during my doctorate. She is much more than a professor to me, she is also a friend.

I am grateful to professor Alexandre Nolasco de Carvalho for everything. He was my first professor at the Universidade de São Paulo and he always encourages me. He taught me a lot during my master and PhD courses. I admire him as a mathematician and he inspires me to improve my knowledge and my career as a researcher.

I appreciate the opportunity to learn a lot with the professors Martin Bohner, István Györi, Plácido Táboas, Miguel Frasson, Everaldo Bonotto and Daná Franková during my doctorate and to collaborate with them. It was a pleasure to me.

I am grateful to FAPESP and CAPES for the financial support during my doctorate. 


\section{Abstract}

The aim of this work is to investigate and develop the theory of impulsive functional dynamic equations on time scales. We prove that these equations represent a special case of impulsive measure functional differential equations. Moreover, we present a relation between these equations and measure functional differential equations and, also, a correspondence between them and generalized ordinary differential equations. Also, we clarify the relation between measure functional differential equations and functional dynamic equations on time scales.

We obtain results on the existence and uniqueness of solutions, continuous dependence on parameters, non-periodic and periodic averaging principles and stability results for all these types of equations. Moreover, we prove some properties concerning regulated functions and equiregulated sets in a Banach space which were essential to our purposes.

The new results presented in this work are contained in 7 papers, two of which have already been published and one accepted. See [16], [32], [34], [36], [37], [38] and [84]. 


\section{Resumo}

O objetivo deste trabalho é investigar e desenvolver a teoria de equações dinâmicas funcionais impulsivas em escalas temporais. Mostramos que estas equações representam um caso especial de equações diferenciais funcionais em medida impulsivas. Também, apresentamos uma relação entre estas equações e as equações diferenciais funcionais em medida e, ainda, mostramos uma relação entre elas e as equações diferenciais ordinárias generalizadas. Relacionamos, também, as equações diferenciais funcionais em medida e as equações dinâmicas funcionais em escalas temporais.

Obtemos resultados sobre existência e unicidade de soluções, dependência contínua, método da média periódico e não-periódico bem como resultados de estabilidade para todos os tipos de equações descritos anteriormente. Também, provamos algumas propriedades relativas às funções regradas e aos conjuntos equiregrados em espaços de Banach, que foram essenciais para os nossos propósitos.

Os resultados novos apresentados neste trabalho estão contidos em 7 artigos, dos quais dois já foram publicados e um aceito. Veja [16], [32], [34], [36], [37], [38] e [84]. 


\section{Contents}

Introduction $\quad 1$

1 Regulated functions $\quad 9$

1.1 Characterizations of compact sets in $G\left([a, b], \mathbb{R}^{n}\right) \ldots \ldots \ldots 14$

1.2 Characterizations of compact sets in $G([a, b], X) \ldots \ldots \ldots$

2 Generalized ODEs $\quad 23$

2.1 The Kurzweil integral . . . . . . . . . . . . . . . . . . . . . . 23

2.2 Generalized ODEs . . . . . . . . . . . . . . . . . 30

3 Dynamic equations on time scales 33

3.1 Time scales calculus . . . . . . . . . . . . . . . . . 34

3.2 Differentiation . . . . . . . . . . . . . . . . . 37

3.3 Darboux and Riemann delta-integrals . . . . . . . . . . . . . . . 40

3.4 Kurzweil-Henstock delta integrals . . . . . . . . . . . . . . . . . . . 48

4 Correspondences between equations $\quad 51$

4.1 Measure FDEs . . . . . . . . . . . . . . . . . . . . . 52

4.2 Measure FDEs and generalized ODEs . . . . . . . . . . . . . 53 
4.3 Measure FDEs and functional dynamic equations on time scales . . . . . . 67

4.4 Impulsive measure FDEs and measure FDEs . . . . . . . . . . . . . . . . 75

4.5 Impulsive functional differential and dynamic equations on time scales . . . 82

5 Existence and uniqueness

5.1 Existence and uniqueness theorem for generalized ODEs . . . . . . . . . 91

5.2 Measure FDEs and functional dynamic equations on time scales . . . . . . 95

5.3 Existence and uniqueness for impulsive equations . . . . . . . . . . . . 100

6 Continuous dependence of solutions on parameters

6.1 Continuous dependence on parameters . . . . . . . . . . . . . . 105

6.2 Continuous dependence of solutions involving variable time scales . . . . . 121

7 Averaging principles

7.1 Non-periodic averaging principles . . . . . . . . . . . . . . . 132

7.1.1 Measure FDEs and functional dynamic equations on time scales . . 133

7.1.2 Impulsive measure FDEs and impulsive functional dynamic equations on time scales . . . . . . . . . . . . . . . . . . 147

7.2 Periodic averaging principles . . . . . . . . . . . . . . . 153

7.2 .1 Generalized ODEs . . . . . . . . . . . . . . 154

7.2.2 Measure FDEs and functional dynamic equations on time scales . . 158

7.2.3 Impulsive measure FDEs and impulsive functional dynamic equations on time scales . . . . . . . . . . . . . . . . . 164

7.3 Examples . . . . . . . . . . . . . . . . . . . . . 169

8 Various types of stability concepts 173

8.1 Stability for measure FDEs . . . . . . . . . . . . . . . 173

8.2 Measure FDEs regarded as generalized ODEs . . . . . . . . . . 176 
8.3 Stability for generalized ODEs . . . . . . . . . . . . . . . 179

8.4 Relations between stability concepts for measure FDEs and generalized ODEs 184

8.5 Stability for impulsive measure FDEs . . . . . . . . . . . . . . . . . 189

8.6 Stability for impulsive functional dynamic equations on time scales _ . . . 195

9 Open and developing problems

203

References 


\section{Introduction}

The theory of time scales is a recent theory and it was developed by Stefan Hilger, on his doctor thesis (see [62]), in order to unify the discrete and continuous analysis. It is worth mentioning that this theory is a powerful tool which does not only unify the discrete and continuous cases, but it also unifies other cases, depending on the chosen time scale.

It is a known fact that many results found in the theory of ordinary differential equations (continuous case) can be easily translated to their analogues in the theory of difference equations (discrete case). However, there are several results for differential equations which are completely different from their analogues for difference equations. In this way, the theory of time scales was developed in order to avoid that some results are proved twice, once for differential equations and another time for difference equations.

The main idea of the theory of dynamic equations on time scales is to prove the results for a type of equations, called dynamic equations, which encompass differential equations and difference equations. This goal is achieved because the domain of the unknown function, called time scale, can be any closed and nonempty subset of the real numbers. Thus, depending on the chosen time scale, we obtain a result for a different type of equation. For instance, if the time scale is the set of the integer numbers, we obtain a result for difference equations. On the other hand, if the time scale is the set of the real numbers, we obtain a result for differential equations.

Other kinds of equations can also be considered. If we choose the time scale to be $q^{\mathbb{Z}}=\left\{q^{z} ; z \in \mathbb{Z}\right\} \cup 0$, where $q>1$, for instance, then we obtain the theory for quantum calculus, which is the basis of quantum physics. Although, these are only some examples, since there are several closed and nonempty subsets of the real numbers (for example, the Cantor set can be a time scale), it is clear that we can have more general results 
depending on the chosen time scale.

For example, a dynamic equation on time scale can model insects populations which are continuous in a certain season, and die out in another one, and, while they die out, their eggs are incubating or dormant, coming to hatch in a different season, giving rise to a nonoverlapping population. See [17], for instance, for details.

Another application of dynamic equations on time scales is the improvement of the study of economic models, since they are much more flexible and realistic models than the ones that are restricted only to the continuous or discrete cases. Also, it is worth noticing that most dynamic optimization problems in economics are described as follows: a consumer has a goal to maximize his lifetime subject utility to certain constraints. Thus, during each period of his life, he should make a decision concerning how much he is willing to give up today to increase consumption tomorrow. This problem is usually described by an optimal control model and its solution is a function which describes optimal behavior for an individual. The solution says how much one should consume during each period of his life in order to ensure that one achieves maximum lifetime consumption. This problem becomes a dynamic one, since a consumer should make decisions not only at one period of his life, but during a sequence of them. In the paper [6], the authors present the above economic problem described by three different models (discrete, continuous and time-scales models). In order to obtain more realistic models, they take into account that a consumer has a limited amount of resources and there is also a trade-off between consuming today and consuming tomorrow.

The discrete model, presented in [6], considers that a consumer has to make a decision about consuming at moments equally spaced, however, we know that in real life, the consumption might occur at unequally spaced moments which turns this model a restricted one. Also, the continuous model presented in [6] does not predict precisely the events which may occur at unequally spaced intervals, not reflecting the reality as well. On the other hand, the time-scales model presented in [6] encompasses the previous ones and gives a more complex analysis concerning the economic models, allowing the hypothesis when the consumption occurs at unequally spaced moments, turning it more realistic. For more details, see [6].

Thus, the theory of time scales has been shown to be a powerful tool to model several economic problems in a more realistic way, since they allow including paychecks, dividend payments and rent payments which usually occurs at unequally spaced points of time.

However, it is difficult to deal directly with the theory of dynamic equations on time 
scales, since it involves a new calculus and tools which are unusual to us. The same happens when we try to study difference equations. The difficulties come from the fact that we are working with another calculus with unusual tools. Due to this fact, many researchers tried different ways to relate difference equations with other types of equations in order to facilitate the obtention of good results for these equations.

In 1983, A. B. Mingarelli, in his manuscript [85], investigated the possibility to obtain a correspondence between difference equations and measure differential equations (VolterraStieltjes equations). His work emphasizes the study of second order equations. Later, in 1994, I. Györi, in his work [51], described an approach to delay difference equations (discrete case) by delay differential equations in order to improve the development of the theory of discrete equations. The result presented in his work is useful to researchers who deal with difference equations. Later, in 2012, A. Slavík extended these relations. Instead of relating only discrete equations with other types of equations, he obtained a more general correspondence, describing a correspondence between dynamic equations on time scales and measure differential equations. See [98], for instance. As we mentioned before, dynamic equations on time scales encompass difference equations and, thus, the correspondence presented by A. Slavík generalizes the previous ones.

In the present work, our main goal is to establish the theory concerning impulsive functional dynamic equations on time scales. This is a recent theory and it generalizes the classical theory of impulsive functional differential equations, which is described extensively in the literature. Our interest in developing this theory lies on the fact that it is a powerful tool for applications, because the most realistic and deterministic models are frequently described by equations involving delays, which apply the causality principle and involve a lag between cause and effect.

In order to facilitate our objective, we focus our attention on the development of the theory of functional dynamic equations on time scales. Inspired by Slavík's paper [98], we investigated the extension of the correspondence presented by him to other types of equations. In this direction, in 2012, we described a correspondence between measure functional differential equations and functional dynamic equations on time scales, which can be found in [36]. With this correspondence, we were able to establish some interesting results for functional dynamic equations on time scales, such as existence and uniqueness of solutions, continuous dependence of solution on the initial data, non-periodic and periodic averaging principles and also stability results.

Notice that functional dynamic equations on time scales are really important tools for applications, since they encompass functional differential equations and extend them, 
allowing more realistic models in several fields of knowledge. The theory about these equations is really scarce, since they were introduced in the literature recently and there are several results to be developed.

Also, the literature concerning measure functional differential equations is not welldeveloped yet. By the way, measure functional differential equation can be described by equations of the form

$$
D x=f\left(x_{t}, t\right) D g, \quad t \in\left[t_{0}, t_{0}+\sigma\right],
$$

where $\sigma, r>0, x_{t}(\theta)=x(t+\theta)$, for $\theta \in[-r, 0], D x$ and $D g$ are the distributional derivatives in the sense of $\mathrm{L}$. Schwartz with respect to $x$ and $g$. The integral form of $(0.0 .1)$ is given by

$$
x(t)=x\left(t_{0}\right)+\int_{t_{0}}^{t} f\left(x_{s}, s\right) \mathrm{d} g(s), \quad t \in\left[t_{0}, t_{0}+\sigma\right] .
$$

We point out that measure functional differential equations are more general than functional differential equations and they encompass functional dynamic equations on time scales, depending on the chosen function $g$. These are good reasons to study measure functional differential equations. In Chapter 4, we describe with more details all these types of equations.

Because functional dynamic equations on time scales are not simple to deal with, we established a correspondence between the solutions of a measure functional differential equation and the solutions of a generalized ordinary differential equation (See [36] and Chapter 4 of this work). It turns out that the framework of abstract generalized ordinary differential equations is much simpler to deal with than the above specific setting of differential equations given by (0.0.1). Therefore, this correspondence facilitates the development of the theory of functional dynamic equations on time scales.

With the correspondences between the solutions of a functional dynamic equation on time scales and the solutions of a measure functional differential equation and these last ones and the solutions of a generalized ordinary differential equation, we were able to prove several interesting results for these types of equations, such as local existence and uniqueness of solutions, continuous dependence of solutions on parameters and periodic averaging principles. All these results can be found in [36] and they will be described with more details in Chapters 5, 6 and 7.

After this, we decided to improve the class of equations on time scales which we were dealing with. Since impulsive differential equations are powerful tools for applications and, 
using them, it is possible to model systems involving controls and jumps in a more realistic way, we proved that the measure functional differential equations that we were dealing with are general enough to encompass impulsive behavior. Thus, we were able to relate measure functional differential equations and impulsive measure functional differential equations (see [37] and Chapter 4). And, using this fact, we could prove a relation between these last ones and impulsive functional dynamical equations on time scales (see Chapter 4).

Thus, we obtained an indirect correspondence between impulsive functional dynamic equations on time scales and generalized differential equations, so that we were able to extend all the results concerning existence and uniqueness, continuous dependence and periodic averaging for impulsive measure functional differential equations and impulsive functional dynamic equations on time scales. All these results can be found in [37] and will be described in Chapters 5, 6 and 7 .

Moreover, using these correspondences, we proved non-periodic averaging principles for measure functional differential equations, functional dynamic equations on time scales, impulsive measure functional differential equations and impulsive functional dynamic equations on time scales. See [34] and Chapter 7, for instance. Later, in [38], we developed stability results for all these equations. Such results are described in Chapter 8. Moreover, we also proved a continuous dependence result for impulsive functional dynamic equations on time scales for variable time scales. See [16] and Chapter 6, for instance.

The present work is divided in nine chapters and it is organized as follows. In the first chapter, we present the basic concepts and main properties of regulated functions and equiregulated sets in $\mathbb{R}^{n}$ and we extend them for general Banach spaces. Also, we present a version of Arzelá-Ascoli Theorem for regulated functions taking values in a Banach space. Almost of all results presented in this chapter are new and they can be found in $[32]$.

The second and third chapters are devoted to presenting the necessary preliminaries to the understanding of the results described in this thesis. The second chapter concerns Kurzweil integration and generalized ordinary differential equations and the third one deals with the theory of dynamic equations on time scales. Readers familiar with these theories can skip these chapters and follow directly to Chapter 4.

The fourth chapter is divided in five sections. The first one is devoted to introducing measure functional differential equations and their properties. The second section brings a correspondence between the solutions of a measure functional differential equation and 
the solutions of a generalized ordinary differential equation. The third section presents a correspondence between the solutions of a measure functional differential equation and the solutions of a functional dynamic equation on time scales. In the fourth section, we prove that measure functional differential equations are general enough to encompass impulsive behavior. In other words, we prove that impulsive measure functional differential equations represent a special type of measure functional differential equations. Finally, in the last section, we establish a correspondence between the solutions of an impulsive measure functional differential equation and the solutions of an impulsive functional dynamic equations on time scales. This chapter contains the most important results of the present work and, by using them, we are able to prove all the results presented in the following chapters. Almost all the results presented in this chapter are original and they can be found in [36] and [37].

The fifth chapter concerns local existence and uniqueness theorems for generalized ordinary differential equations, measure functional differential equations, impulsive measure functional differential equations, functional dynamic equations on time scales and impulsive functional dynamic equations on time scales. Almost all the results of this chapter are new and they can be found in [36] and [37].

The sixth chapter is divided in two sections. In the first one, we present results on continuous dependence of solutions on the initial data for generalized ordinary differential equations, measure functional differential equations, functional dynamic equations on time scales, impulsive measure functional differential equations and impulsive functional dynamic equations on time scales. In the second section, we present results on continuous dependence of solutions involving variable time scales for impulsive functional dynamic equations on time scales. Most of the results presented in this chapter are new and can be found in [16], [32], [36] and [37].

Chapter 7 is devoted to averaging principles. It is divided in three sections. The first one concerns non-periodic averaging principles for measure functional differential equations, functional dynamic equations on time scales, impulsive measure functional differential equations and impulsive functional dynamic equations on time scales. The second section presents periodic averaging principle for generalized ordinary differential equations, measure functional differential equations, functional dynamic equations on time scales, impulsive measure functional differential equations and impulsive functional dynamic equations on time scales. Finally, in the last section, we present some examples to illustrate the averaging principles. Almost all results presented in this chapter are new and they can be found in [34], [36], [37] and [84]. 
In Chapter 8, we present new concepts of stability of the trivial solution of measure functional differential equations, functional dynamic equations on time scales, impulsive measure functional differential equations, impulsive functional dynamic equations on time scales and generalized ordinary differential equations, as well the relations between the various concepts of stability. All the results presented in this chapter are new and they are contained in [38].

In the last chapter, we present several open and developing problems which may motivate future and interesting projects. 



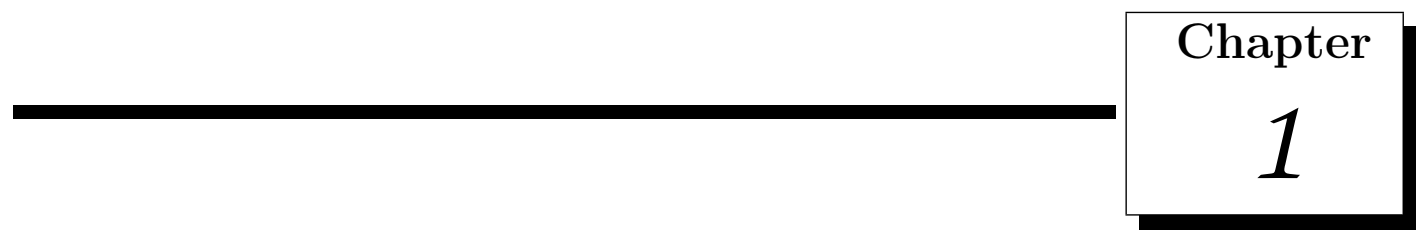

\section{Regulated functions}

In this chapter, we state and prove some fundamental results concerning regulated functions which will be essential to our purposes. The majority of the results presented here are new and they can be found in [32]. The basic references to this chapter are [32], [46], [64] and [94].

Let $X$ be a Banach space with norm $\|\cdot\|$.

A function $f:[a, b] \rightarrow X$ is called regulated, if the lateral limits

$$
\lim _{s \rightarrow t^{-}} f(s)=f\left(t^{-}\right), \quad t \in(a, b], \quad \text { and } \lim _{s \rightarrow t^{+}} f(s)=f\left(t^{+}\right), \quad t \in[a, b)
$$

exist. The space of all regulated functions $f:[a, b] \rightarrow X$ will be denoted by $G([a, b], X)$, and it is a Banach space under the usual supremum norm $\|f\|_{\infty}=\sup _{a \leq t \leq b}\|f(t)\|$. The subspace of all continuous functions $f:[a, b] \rightarrow X$ will be denoted by $C([a, b], X)$. It is clear that $C([a, b], X) \subset G([a, b], X)$ and, in $C([a, b], X)$, we consider the norm induced by $G([a, b], X)$. Moreover, we denote by $G^{-}([a, b], \mathbb{R})$ the set of regulated functions $f$ : $[a, b] \rightarrow X$ which are left-continuous.

The finite sets $d=\left\{t_{0}, t_{1}, \ldots, t_{m}\right\}$ of points in the closed interval $[a, b]$ such that $a=t_{0}<t_{1}<\ldots<t_{m}=b$ are called partitions of $[a, b]$. Given a partition of $[a, b]$, its elements are usually denoted by $t_{0}, t_{1}, \ldots, t_{m}$. The set of all partitions of $[a, b]$ is denoted by $\mathcal{D}[a, b]$. Moreover, we call a pair $\left(\tau_{i},\left[t_{i-1}, t_{i}\right]\right)$ by tagged interval, where $\tau_{i} \in\left[t_{i-1}, t_{i}\right]$ is a tag of $\left[t_{i-1}, t_{i}\right]$.

Let $\delta>0$, we say that a partition of $[a, b]$ is $\delta$-fine, if for every $i=1,2, \ldots, m$, we 
have $\left|t_{i}-t_{i-1}\right|<\delta$. And also, a tagged interval $\left(\tau_{i},\left[t_{i-1}, t_{i}\right]\right)$ with $\tau_{i} \in\left[t_{i-1}, t_{i}\right]$ is $\delta$-fine, if $\left[t_{i-1}, t_{i}\right] \subset\left(\tau_{i}-\delta\left(\tau_{i}\right), \tau_{i}+\delta\left(\tau_{i}\right)\right)$. For more details, the reader may consult Chapter 2 or $[94]$.

A function $f:[a, b] \rightarrow X$ is called a finite step function, if there is a finite partition $a=t_{0}<t_{1}<\ldots<t_{m}=b$ such that in every open interval $\left(t_{i-1}, t_{i}\right), i=1, \ldots, m$, the function is identically equal to an element $c_{i} \in X$.

Given a function $f:[a, b] \rightarrow X$, its variation $\operatorname{var}_{a}^{b} f$ on the interval $[a, b]$ is defined by

$$
\operatorname{var}_{a}^{b} f=\sup _{d \in \mathcal{D}[a, b]} \sum_{i=1}^{m}\left\|f\left(t_{i}\right)-f\left(t_{i-1}\right)\right\|,
$$

that is, the supremum is taken over all $\delta$-fine partitions $d=\left\{t_{0}, t_{1}, \ldots, t_{m}\right\} \in \mathcal{D}[a, b]$ of $[a, b]$. If $\operatorname{var}_{a}^{b} f<\infty$, we say that $f$ is of bounded variation on the interval $[a, b]$. Also, $B V([a, b], X)$ denotes the Banach space of functions $f:[a, b] \rightarrow X$ of bounded variation on $[a, b]$, equipped with the variation norm

$$
f \in B V([a, b], X) \mapsto\|f\|_{B V}=\|f(a)\|+\operatorname{var}_{a}^{b} f
$$

It is a well-known fact that every function which is regulated on $[a, b]$ is bounded on this interval and is the uniform limit of finite step functions. See [64], for instance. Moreover, every function of bounded variation on $[a, b]$ is regulated on $[a, b]$.

A set $\mathcal{A} \subset G([a, b], X)$ is called equiregulated, if it has the following property: for every $\varepsilon>0$ and $t_{0} \in[a, b]$, there is $\delta>0$ such that

1. If $x \in \mathcal{A}, t^{\prime} \in[a, b]$ and $t_{0}-\delta<t^{\prime}<t_{0}$, then $\left\|x\left(t_{0}{ }^{-}\right)-x\left(t^{\prime}\right)\right\|<\varepsilon$.

2. If $x \in \mathcal{A}, t^{\prime \prime} \in[a, b]$ and $t_{0}<t^{\prime \prime}<t_{0}+\delta$, then $\left\|x\left(t^{\prime \prime}\right)-x\left(t_{0}+\right)\right\|<\varepsilon$.

The next result describes a property of regulated functions. It is a generalization of Proposition 1.9 from [46] for Banach space-valued functions and its proof follows the same steps as the proof of Proposition 1.9 from [46].

Lemma 1.1. Let $x:[a, b] \rightarrow X$ be a regulated function. Then for every $\varepsilon>0$, there is $a$ $\delta$-fine partition

$$
a=t_{0}<t_{1}<\ldots<t_{n}=b
$$

such that

$$
\left\|x\left(t^{\prime \prime}\right)-x\left(t^{\prime}\right)\right\|<\varepsilon,
$$


for $t_{i-1}<t^{\prime}<t^{\prime \prime}<t_{i}$ and $i=1,2, \ldots, n$.

Proof. Let $\varepsilon>0$ be given and denote by $C$ the set of all $\tau \in(a, b]$ such that there is a $\delta$-fine partition on $[a, \tau], a=t_{0}<t_{1}<\ldots<t_{k}=\tau$, satisfying (1.0.2) with $k$ instead of $n$.

Since $x$ is regulated, the limit $\lim _{t \rightarrow a^{+}} x(t)=x\left(a^{+}\right)$exists and it guarantees the existence of $\gamma>0$ such that

$$
\left\|x(t)-x\left(a^{+}\right)\right\|<\varepsilon / 2, \text { for } t \in(a, \gamma)
$$

Then, for every $a<t^{\prime}<t^{\prime \prime}<\gamma$, we get

$$
\left\|x\left(t^{\prime \prime}\right)-x\left(t^{\prime}\right)\right\| \leq\left\|x\left(t^{\prime \prime}\right)-x\left(a^{+}\right)\right\|+\left\|x\left(t^{\prime}\right)-x\left(a^{+}\right)\right\|<\varepsilon
$$

As a result, we obtain $\gamma \in C$.

Denote $c=\sup C$. Then $c>a$.

Since the limit $x\left(c^{-}\right)=\lim _{t \rightarrow c^{-}} x(t)$ exists, there is $\delta>0$ such that $\left\|x(t)-x\left(c^{-}\right)\right\|<$ $\varepsilon / 2$, for every $t \in(c-\delta, c)$. Take $\beta \in C \cap(c-\delta, c)$. Since $\beta \in C$, there is a finite sequence $a=t_{0}<t_{1}<\ldots<t_{k}=\beta$ such that (1.0.2) holds with $k$ instead $n$.

Without loss of generality, we can take $t_{k+1}>c$. Then

$$
\left\|x\left(t^{\prime \prime}\right)-x\left(t^{\prime}\right)\right\| \leq\left\|x\left(t^{\prime \prime}\right)-x\left(c^{-}\right)\right\|+\left\|x\left(t^{\prime}\right)-x\left(c^{-}\right)\right\|<\varepsilon,
$$

for $t_{k}=\beta<t^{\prime}<t^{\prime \prime}<c<t_{k+1}$ which implies that (1.0.2) holds also for $n=k+1$. Therefore $c \in C$.

Using the same idea as in the beginning of this proof, it can be shown that if $c<b$, then there is $t>c$ which belongs to $C$. However, this contradicts the fact that $c$ is the supremum of $C$. Therefore $c=b$ and we obtain the desired result.

The next result gives a characterization of equiregulated functions for the Banach space-valued case. It is a new result and it can be found in [32]. The proof is inspired in Lemma 2.1 from [46].

Theorem 1.2. A set $\mathcal{A} \subset G([a, b], X)$ is equiregulated, if and only if, for every $\varepsilon>0$ there is a $\delta$-fine partition $a=t_{0}<t_{1}<\ldots<t_{n}=b$ such that

$$
\left\|x\left(t^{\prime}\right)-x(t)\right\| \leq \varepsilon
$$


for every $x \in \mathcal{A}$ and $\left[t, t^{\prime}\right] \subset\left(t_{j-1}, t_{j}\right)$, for $j=1,2, \ldots, n$.

Proof. $(\Rightarrow)$ Let $\varepsilon>0$ be given and let $D$ be the set of all $\gamma \in(a, b]$ such that there is a partition $a=t_{0}<t_{1}<\ldots<t_{k}=\gamma$ for which (1.0.3) holds with $k$ instead of $n$.

Since $\mathcal{A}$ is equiregulated, there is $\delta_{1} \in(0, b-a]$ such that $\left\|x(t)-x\left(a^{+}\right)\right\| \leq \varepsilon / 2$, for every $x \in \mathcal{A}$ and $t \in\left(a, a+\delta_{1}\right)$. Denote $d_{1}=a+\delta_{1}, a=t_{0}<t_{1}=d_{1}$. Thus, for $\left[t, t^{\prime}\right] \subset\left(a, d_{1}\right)$ and $x \in \mathcal{A}$, the inequalities

$$
\left\|x(t)-x\left(t^{\prime}\right)\right\| \leq\left\|x(t)-x\left(a^{+}\right)\right\|+\left\|x\left(t^{\prime}\right)-x\left(a^{+}\right)\right\| \leq \varepsilon
$$

holds and we have $d_{1} \in D$.

Let $\tilde{d}$ be the supremum of set $D$. Since $x \in \mathcal{A}$, then there is $\delta>0$ such that

$$
\left\|x\left(\tilde{d}^{-}\right)-x(t)\right\| \leq \frac{\varepsilon}{2}
$$

for every $x \in \mathcal{A}$ and $t \in(\tilde{d}-\delta, \tilde{d}) \cap[a, b]$.

Let $d \in D \cap(\tilde{d}-\delta, \tilde{d})$ and a partition $a=t_{0}<t_{1}<\ldots<t_{k}=d$ be such that (1.0.3) holds with $k$ instead of $n$. Denote $t_{k+1}=\tilde{d}$. Then for $\left[t, t^{\prime}\right] \subset\left(t_{k}, t_{k+1}\right)$ and $x \in \mathcal{A}$, we have

$$
\left\|x(t)-x\left(t^{\prime}\right)\right\| \leq\left\|x(t)-x\left(\tilde{d}^{-}\right)\right\|+\left\|x\left(t^{\prime}\right)-x\left(\tilde{d}^{-}\right)\right\| \leq \varepsilon
$$

which implies $\tilde{d} \in D$. Thus we have two possibilities: either $\tilde{d}=b$ or $\tilde{d}<b$. In the first case, we finish the proof. On the other hand, for the second case, we can use a similar argument as before to find $d_{2} \in(\tilde{d}, b]$ such that $d_{2} \in D$ and, in this case, this contradicts the fact that $\tilde{d}=\sup D$. Thus $\tilde{d}=b$ and we finished the proof.

$(\Leftarrow)$ Reciprocally, given $\varepsilon>0$, there is a $\delta$-fine partition $a=t_{0}<t_{1}<\ldots<t_{k}=b$ such that

$$
\left\|x\left(t^{\prime}\right)-x(t)\right\| \leq \varepsilon
$$

for every $x \in \mathcal{A}$ and $\left[t, t^{\prime}\right] \subset\left(t_{j-1}, t_{j}\right)$, for $j=1,2, \ldots, k$.

Let $\tau_{j}$ be a tag of $\left(t_{j-1}, t_{j}\right)$. Since $\left(t_{j-1}, t_{j}\right)$ is $\delta$-fine, it can be rewritten by $\left(\tau_{j}-\delta, \tau_{j}+\delta\right)$. Therefore, the inequality (1.0.4) holds, for $t, t^{\prime} \in\left(\tau_{j}-\delta, \tau_{j}+\delta\right)$. Taking $t=\tau_{j}^{-}$and $t^{\prime} \in\left(\tau_{j}-\delta, \tau_{j}\right]$, then the inequality (1.0.4) remains true. Also, if $t=\tau_{j}^{+}$and $t^{\prime} \in\left[\tau_{j}, \tau_{j}+\delta\right)$, the inequality (1.0.4) is fulfilled. Then, by definition, it follows that $\mathcal{A}$ is equiregulated.

The next result describes a property of an equiregulated set $\mathcal{A} \subset G([a, b], X)$. The 
proof of this result is inspired in [46], Lemma 2.2 .

Theorem 1.3. Assume that a set $\mathcal{A} \subset G([a, b], X)$ is equiregulated and for any $t \in[a, b]$ there is a number $\gamma_{t}$ such that, for $x \in \mathcal{A}$,

$$
\left\|x(t)-x\left(t^{-}\right)\right\| \leq \gamma_{t}, t \in(a, b] \quad \text { and } \quad\left\|x\left(t^{+}\right)-x(t)\right\| \leq \gamma_{t}, t \in[a, b) .
$$

Then there is a constant $K>0$ such that $\|x(t)-x(a)\| \leq K$, for every $x \in \mathcal{A}$ and $t \in[a, b]$.

Proof. Let $B$ be the set of all $\tau \in(a, b]$ such that there is $K_{\tau}>0$ such that

$$
\|x(t)-x(a)\| \leq K_{\tau}
$$

for any $x \in \mathcal{A}$ and $t \in[a, \tau]$.

Since the set $\mathcal{A}$ is equiregulated, there is $\delta>0$ such that

$$
\left\|x(t)-x\left(a^{+}\right)\right\| \leq 1
$$

for every $x \in \mathcal{A}$ and $t \in(a, a+\delta]$. This fact together with the hypothesis imply that

$$
\|x(t)-x(a)\| \leq\left\|x(t)-x\left(a^{+}\right)\right\|+\left\|x\left(a^{+}\right)-x(a)\right\| \leq 1+\gamma_{a}=K_{(a+\delta)},
$$

for every $t \in(a, a+\delta]$ and $x \in \mathcal{A}$. Hence $(a, a+\delta] \subset B$.

Denote $\tau_{0}=\sup B$. As a consequence of the equiregulatedness of $\mathcal{A}$, there is $\delta^{\prime}>0$ such that $\left\|x(t)-x\left(\tau_{0}^{-}\right)\right\| \leq 1$ for every $x \in \mathcal{A}$ and $t \in\left[\tau_{0}-\delta^{\prime}, \tau_{0}\right)$.

Let $\tau \in B \cap\left[\tau_{0}-\delta^{\prime}, \tau_{0}\right)$. Then

$$
\begin{aligned}
\|x(t)-x(a)\| & \leq\left\|x(t)-x\left(\tau_{0}^{-}\right)\right\|+\left\|x\left(\tau_{0}^{-}\right)-x(\tau)\right\|+\|x(\tau)-x(a)\| \\
& \leq 1+1+K_{\tau}=2+K_{\tau},
\end{aligned}
$$

for $x \in \mathcal{A}$ and $t \in\left(\tau, \tau_{0}\right)$. Also,

$$
\left\|x\left(\tau_{0}^{-}\right)-x(a)\right\| \leq\left\|x\left(\tau_{0}^{-}\right)-x(\tau)\right\|+\|x(\tau)-x(a)\| \leq 1+K_{\tau} .
$$

These inequalities and the hypotheses imply

$$
\left\|x\left(\tau_{0}\right)-x(a)\right\| \leq\left\|x\left(\tau_{0}\right)-x\left(\tau_{0}^{-}\right)\right\|+\left\|x\left(\tau_{0}^{-}\right)-x(a)\right\| \leq \gamma_{\tau_{0}}+1+K_{\tau} .
$$


Thus $\tau_{0} \in B$, where $K_{\tau_{0}}=\gamma_{\tau_{0}}+1+K_{\tau}$.

If $\tau_{0}<b$, then since $\mathcal{A}$ is equiregulated, there is $\delta^{\prime \prime}>0$ such that

$$
\left\|x(t)-x\left(\tau_{0}^{+}\right)\right\| \leq 1, \quad \text { for any } x \in \mathcal{A}, \quad t \in\left(\tau_{0}, \tau_{0}+\delta^{\prime \prime}\right],
$$

which implies

$$
\begin{aligned}
\|x(t)-x(a)\| & \leq\left\|x(t)-x\left(\tau_{0}^{+}\right)\right\|+\left\|x\left(\tau_{0}^{+}\right)-x\left(\tau_{0}\right)\right\|+\left\|x\left(\tau_{0}\right)-x(a)\right\| \leq \\
& \leq 1+\gamma_{\tau_{0}}+K_{\tau_{0}}=K_{\left(\tau_{0}+\delta^{\prime \prime}\right)}
\end{aligned}
$$

for $t \in\left(\tau_{0}, \tau_{0}+\delta^{\prime \prime}\right]$ and $x \in \mathcal{A}$. Thus $\tau_{0}+\delta^{\prime \prime} \in B$ which contradicts the fact that $\tau_{0}=\sup B$. Therefore $\tau_{0}=b$ and we have the desired result.

\subsection{Characterizations of compact sets in $G\left([a, b], \mathbb{R}^{n}\right)$}

In this section, we present some properties concerning the compact sets in $G\left([a, b], \mathbb{R}^{n}\right)$. Also, we present a known Arzelá-Ascoli-type theorem for regulated functions taking values in finite dimensional spaces. This result was establish by Daná Fraňková in 1991 in the paper [46] which is the main reference to the present section.

We denote any norm in $\mathbb{R}^{n}$ by $|\cdot|$.

We start this section by presenting a result which describes a necessary and sufficient condition for a subset of $G\left([a, b], \mathbb{R}^{n}\right)$ to be relatively compact. Such result can be found in [46], Proposition 2.3.

Theorem 1.4. A set $\mathcal{A} \subset G\left([a, b], \mathbb{R}^{n}\right)$ is relatively compact, if and only if, it is equiregulated and for any $t \in[a, b]$, there is a number $\gamma_{t}$ such that

$$
\left|x(t)-x\left(t^{-}\right)\right| \leq \gamma_{t}, t \in(a, b], \quad \text { and } \quad\left|x\left(t^{+}\right)-x(t)\right| \leq \gamma_{t}, t \in[a, b) .
$$

and there is $\alpha>0$ such that $|x(a)| \leq \alpha$ for any $x \in \mathcal{A}$.

As an immediate consequence of the previous theorem, we obtain the following result which can be found in [46], Corollary 2.4.

Corollary 1.5. A set $\mathcal{A} \subset G\left([a, b], \mathbb{R}^{n}\right)$ is relatively compact, if and only if, it is equiregulated and, for every $t \in[a, b]$, the set $\{x(t) ; x \in \mathcal{A}\}$ is bounded in $\mathbb{R}^{n}$. 
The next theorem is a crucial result to prove our main results concerning continuous dependence of solutions on parameters in Chapter 6. A proof of it can be found in [46], Theorem 2.14 .

Theorem 1.6. For an arbitrary function $x:[a, b] \rightarrow \mathbb{R}^{n}$, the following conditions are equivalent:

(i) $x$ is a regulated function.

(ii) There is a continuous function $y:[0,1] \rightarrow \mathbb{R}^{n}$ and an increasing function $v:[a, b] \rightarrow$ $[0,1]$ such that $v(a)=0, v(b)=1$ satisfying $x(t)=y(v(t))$ for every $t \in[a, b]$.

(iii) There is an increasing continuous function $\eta:[0,1] \rightarrow[0, \infty), \eta(0)=0$ and an increasing function $v:[a, b] \rightarrow[0,1]$ such that

$$
\left|x\left(t_{2}\right)-x\left(t_{1}\right)\right| \leq \eta\left(v\left(t_{2}\right)-v\left(t_{1}\right)\right)
$$

for every $a \leq t_{1} \leq t_{2} \leq b$.

Remark 1.7. It is worth noticing that every nondecreasing function $v:[a, b] \rightarrow \mathbb{R}$ can be bounded by an increasing function, that is, if $v:[a, b] \rightarrow \mathbb{R}, 0<a<b$, is a nondecreasing function, then the inequality

$$
v(t) \leq v(t)+t=h(t)
$$

is fulfilled, where the function $h$ given above is increasing.

The next result follows as an immediate consequence of Remark 1.7 and Theorem 1.6.

Corollary 1.8. For an arbitrary function $x:[a, b] \rightarrow \mathbb{R}^{n}$, the following conditions are equivalent:

(i) $x$ is a regulated function.

(ii) There is an increasing continuous function $\eta:[0,1] \rightarrow[0, \infty), \eta(0)=0$ and a nondecreasing function $v:[a, b] \rightarrow[0,1]$ such that

$$
\left|x\left(t_{2}\right)-x\left(t_{1}\right)\right| \leq \eta\left(v\left(t_{2}\right)-v\left(t_{1}\right)\right)
$$

for every $a \leq t_{1} \leq t_{2} \leq b$. 
The following theorem is an essential result to our purposes, since it gives sufficient conditions for a subset of the space of regulated function to be equiregulated and to satisfy condition (1.1.1).

Theorem 1.9 ([45], Theorem 2.17). For any set $\mathcal{A} \subset G\left([a, b], \mathbb{R}^{n}\right)$, the following properties are equivalent:

(i) $\mathcal{A}$ is equiregulated and satisfies (1.1.1);

(ii) There is an increasing function $v:[a, b] \rightarrow[0,1]$ such that $v(a)=0, v(b)=1$ and an increasing continuous function $\eta:[0,1] \rightarrow[0, \infty), \eta(0)=0$ such that

$$
\left|x\left(t^{\prime \prime}\right)-x\left(t^{\prime}\right)\right| \leq \eta\left(v\left(t^{\prime \prime}\right)-v\left(t^{\prime}\right)\right),
$$

for every $x \in \mathcal{A}, a \leq t^{\prime}<t^{\prime \prime} \leq b$.

(iii) There is an increasing function $v:[a, b] \rightarrow[0,1]$ such that $v(a)=0, v(b)=1$ and an equicontinuous set $\mathcal{B} \subset C\left([0,1], \mathbb{R}^{n}\right)$ such that $\mathcal{A} \subset \mathcal{B} \circ v$, that is, for every $x \in \mathcal{A}$ there is a continuous function $y \in \mathcal{B}$ such that $x=y \circ v$.

The next result is a generalization of the Arzelá-Ascoli theorem for regulated functions. It is the most important result of this section and it can be found in [46], Theorem 2.18.

Theorem 1.10. For any set of regulated functions $\mathcal{A} \subset G\left([a, b], \mathbb{R}^{n}\right)$, the following properties are equivalent:

(i) $\mathcal{A}$ is relatively compact in $G\left([a, b], \mathbb{R}^{n}\right)$;

(ii) $\mathcal{A}$ is equiregulated, satisfies (1.1.1) and

$$
\text { there is } \alpha>0 \text { such that }|x(0)| \leq \alpha \text { for any } x \in \mathcal{A} \text {. }
$$

(iii) The set $\mathcal{A}$ satisfies (1.1.2) and (1.1.3);

(iv) There is an increasing function $v:[a, b] \rightarrow[0,1]$ such that $v(a)=0, v(b)=1$ and $a$ compact set of continuous functions $\mathcal{B} \subset C\left([0,1], \mathbb{R}^{n}\right)$ such that $\mathcal{A} \subset \mathcal{B} \circ v$.

Using the previous result and Remark 1.7, the next result follows immediately.

Corollary 1.11. The following conditions are equivalent: 
(i) $\mathcal{A} \subset G\left([a, b], \mathbb{R}^{n}\right)$ is relatively compact.

(ii) The set $\{x(a) ; x \in \mathcal{A}\}$ is bounded and there is an increasing continuous function $\eta:[0, \infty) \rightarrow[0, \infty), \eta(0)=0$ and a nondecreasing function $v:[a, b] \rightarrow \mathbb{R}$ such that

$$
\left|x\left(t_{2}\right)-x\left(t_{1}\right)\right| \leq \eta\left(v\left(t_{2}\right)-v\left(t_{1}\right)\right)
$$

for every $x \in \mathcal{A}, a \leq t_{1} \leq t_{2} \leq b$.

(iii) $\mathcal{A}$ is equiregulated and for every $t \in[a, b]$, the set $\{x(t) ; x \in \mathcal{A}\}$ is bounded.

\subsection{Characterizations of compact sets in $G([a, b], X)$}

In this section, we investigate an extension of Arzelá-Ascoli Theorem for regulated functions taking values in a general Banach space $X$. Throughout this section, we will consider that $X$ is a Banach space endowed with norm $\|\cdot\|$.

All the results presented here are new and they can be found in [32].

One cannot expect to prove a version of Corollary 1.11 for a general Banach space using similar hypotheses, because the equiregulatedness of $\mathcal{A} \subset G([a, b], X)$ and the boundedness of the set $\{x(t) ; x \in \mathcal{A}\}$ in $X$, for every $t \in[a, b]$, are not sufficient to guarantee that a set $\mathcal{A}$ is relatively compact. Below, we present an example which illustrates this fact.

Example 1.12. Let $[a, b]=[0,1]$ and $Z \subset X$ be a set which is bounded, but not relatively compact in $X$. Given $\varepsilon>0$, there is a sequence $\left\{z_{n} ; n \in \mathbb{N}\right\} \subset Z$ such that $\left\|z_{n}\right\| \leq K$ and $\left\|z_{n}-z_{m}\right\| \geq \varepsilon$, for some constant $K>0$ and for some $n \neq m$. Therefore the set $B=\left\{y_{n}:[0,1] \rightarrow X, y_{n}(t)=t z_{n} ; n \in \mathbb{N}\right\}$ is bounded, since $z_{n}$ is bounded. Also, by definition, $B$ is equiregulated and $\left\{y_{n}(0) ; n \in \mathbb{N}\right\}$ is relatively compact in $X$. But $B$ is not relatively compact in $G([0,1], X)$.

However, we can add one hypothesis in order to guarantee that the set $\mathcal{A}$ is relatively compact. For instance, we can suppose for every $t \in[a, b]$, the set $\{x(t) ; x \in \mathcal{A}\}$ is relatively compact in $X$. In this way, $\mathcal{A}$ is relatively compact in $G([a, b], X)$. This is the content of the next result which is a type of Árzela-Ascoli theorem for regulated functions taking values in a Banach space. Such result is original and can be found in [32].

Theorem 1.13. Let $\mathcal{A} \subset G([a, b], X)$ be equiregulated and, for every $t \in[a, b]$, let the set $\{x(t) ; x \in \mathcal{A}\}$ be relatively compact in $X$. Then the set $\mathcal{A}$ is relatively compact in $G([a, b], X)$. 
Proof. Let $\left\{x_{n} ; n \in \mathbb{N}\right\} \subset \mathcal{A}$ be a given sequence.

Since $\mathcal{A}$ is equiregulated, given $\varepsilon>0$, there is a $\delta$-fine partition $a=\alpha_{0}<\alpha_{1}<\ldots<$ $\alpha_{m}=b$ such that

$$
\left\|x_{n}\left(t^{\prime}\right)-x_{n}(t)\right\|<\varepsilon
$$

for every $n \in \mathbb{N}$ and $\alpha_{i-1}<t<t^{\prime}<\alpha_{i}$.

By assumption, the set $\left\{x_{n}\left(\alpha_{i}\right), n \in \mathbb{N}\right\}$ is relatively compact in $X$ for every $i=$ $0,1,2, \ldots, m$. Therefore we can find a subsequence of indexes $\left\{n_{k} ; k \in \mathbb{N}\right\} \subset\{n ; n \in \mathbb{N}\}$ satisfying $n_{k} \in \mathbb{N}, n_{k+1}>n_{k}$ such that the set

$$
\left\{x_{n_{k}}\left(\alpha_{i}\right), k \in \mathbb{N}\right\}
$$

is also relatively compact in $X$ for every $i$.

Using this fact, we can find the elements $\left\{y_{i} ; i=0,1,2, \ldots, m\right\} \subset X$ such that $y_{i}=$ $\lim _{k \rightarrow \infty} x_{n_{k}}\left(\alpha_{i}\right)$. Therefore, there exists $N \in \mathbb{N}$ such that for every $k>N$, we have

$$
\left\|x_{n_{k}}\left(\alpha_{i}\right)-y_{i}\right\|<\frac{\varepsilon}{4}
$$

Let $q>k$, then

$$
\left\|x_{n_{q}}\left(\alpha_{i}\right)-y_{i}\right\|<\frac{\varepsilon}{4}
$$

for any $i=0,1,2, \ldots, m$.

Now, let $t \in[a, b]$ and $q \in \mathbb{N}$ be such that $q>k$. Then either $t=\alpha_{i}$ for some $i \in\{0,1,2, \ldots, m\}$ and, in this case, we have

$$
\left\|x_{n_{k}}(t)-x_{n_{q}}(t)\right\| \leq\left\|x_{n_{k}}\left(\alpha_{i}\right)-y_{i}\right\|+\left\|x_{n_{q}}\left(\alpha_{i}\right)-y_{i}\right\|<\frac{\varepsilon}{2}
$$

or $t \in\left(\alpha_{i-1}, \alpha_{i}\right)$ for some $i \in\{1, \ldots, m\}$ and, in this case, we have

$$
\begin{aligned}
\left\|x_{n_{k}}(t)-x_{n_{q}}(t)\right\| & \leq\left\|x_{n_{k}}(t)-x_{n_{k}}\left(\alpha_{i-1}\right)\right\|+\left\|x_{n_{q}}(t)-x_{n_{q}}\left(\alpha_{i-1}\right)\right\|+\left\|x_{n_{k}}\left(\alpha_{i-1}\right)+x_{n_{q}}\left(\alpha_{i-1}\right)\right\| \\
& <\frac{\varepsilon}{4}+\frac{\varepsilon}{4}+\frac{\varepsilon}{2}=\varepsilon .
\end{aligned}
$$

Thus, for $t \in[a, b]$, the sequence $\left\{x_{n_{k}}(t) ; k \in \mathbb{N}\right\} \subset X$ satisfies the Cauchy condition. Since the space $X$ is complete and the sequence $\left\{x_{n_{k}}(t) ; k \in \mathbb{N}\right\}$ is a Cauchy sequence, then $\lim _{k \rightarrow \infty} x_{n_{k}}(t)$ exists. Let $x_{0}(t)=\lim _{k \rightarrow \infty} x_{n_{k}}(t)$. Therefore $x_{n_{k}} \rightrightarrows x_{0}$ on $[a, b]$, which means that $x_{0}$ is the limit of the subsequence $\left\{x_{n_{k}}\right\}$ in $G([a, b], X)$. Hence any sequence $\left\{x_{n}\right\} \subset \mathcal{A}$ 
has a convergent subsequence which means that $\mathcal{A}$ is relatively compact.

The next result concerns an important property of an equiregulated sequence of Banach space-valued functions. It is an original result which can be found in [32].

Lemma 1.14. Let $\left\{x_{k}\right\}_{k=1}^{\infty}$ be a sequence of functions from $[a, b]$ to $X$. If the sequence $\left\{x_{k}\right\}_{k=1}^{\infty}$ converges pointwisely to $x_{0}$ and the sequence $\left\{x_{k}\right\}_{k=1}^{\infty}$ is equiregulated, then $\left\{x_{k}\right\}_{k=1}^{\infty}$ converges uniformly to $x_{0}$.

Proof. Since the sequence of functions $\left\{x_{k}\right\}_{k=1}^{\infty}$ is equiregulated, we obtain, by Theorem 1.2 , that given $\varepsilon>0$, there exist a $\delta$-fine partition $a=s_{0}<s_{1}<\ldots<s_{m}=b$ such that

$$
\left\|x_{k}(t)-x_{k}(s)\right\|<\varepsilon / 3
$$

for $k \in \mathbb{N}$ and $s_{i-1}<s<t<s_{i}$.

Let $\tau_{i} \in\left(s_{i-1}, s_{i}\right)$. Since the sequence $x_{k}$ converges pointwisely to $x_{0}$, it follows that $x_{k}\left(s_{i}\right) \rightarrow x_{0}\left(s_{i}\right)$ and $x_{k}\left(\tau_{i}\right) \rightarrow x_{0}\left(\tau_{i}\right)$, for every $i=1,2, \ldots, m$. Therefore, given $\varepsilon>0$, there exists $k_{0} \in \mathbb{N}$ such that if $k>k_{0}$, we have

$$
\left\|x_{k}\left(s_{i}\right)-x_{0}\left(s_{i}\right)\right\|<\varepsilon / 3 \text { and }\left\|x_{k}\left(\tau_{i}\right)-x_{0}\left(\tau_{i}\right)\right\|<\varepsilon / 3 \text {, }
$$

for every $i=1,2, \ldots, m$.

Now, let $t \in[a, b]$ be given. Thus, either $t=s_{i}$ for some $i$, or $t \in\left(s_{i-1}, s_{i}\right)$ for some $i$. In the first case, we have

$$
\left\|x_{k}(t)-x_{0}(t)\right\|<\varepsilon / 3 .
$$

On the other hand, the second case implies

$$
\left\|x_{k}(t)-x_{0}(t)\right\| \leq\left\|x_{k}(t)-x_{k}\left(\tau_{i}\right)\right\|+\left\|x_{k}\left(\tau_{i}\right)-x_{0}\left(\tau_{i}\right)\right\|+\left\|x_{0}\left(\tau_{i}\right)-x_{0}(t)\right\|<\varepsilon,
$$

which means that $\left\|x_{k}-x_{0}\right\|_{\infty}<\varepsilon$ and hence $x_{k} \rightarrow x_{0}$ uniformly on $[a, b]$ and we have the desired result.

The next lemma is a new result and it will be essential to our purposes.

Lemma 1.15. Let $\left\{x_{k}\right\}_{k=1}^{\infty}$ be a sequence of functions from $[a, b]$ to $X$.

(i) If the sequence of functions $x_{k}$ converges uniformly to $x_{0}$ as $k \rightarrow \infty$ on $[a, b]$, then $x_{k}\left(t^{-}\right) \rightarrow x_{0}\left(t^{-}\right)$, for $t \in(a, b]$, and $x_{k}\left(t^{+}\right) \rightarrow x_{0}\left(t^{+}\right)$, for $t \in[a, b)$. 
(ii) Assume that the sequence of functions $\left\{x_{k}\right\}_{k=1}^{\infty}$ converges pointwisely to $x_{0}$ as $k \rightarrow \infty$ on $[a, b]$ and $x_{k}\left(t^{-}\right) \rightarrow x_{0}\left(t^{-}\right)$, for $t \in(a, b]$, and $x_{k}\left(t^{+}\right) \rightarrow x_{0}\left(t^{+}\right)$, for $t \in[a, b)$. If $x_{0}$ is a regulated function, then the sequence $\left\{x_{k}\right\}_{k=1}^{\infty}$ converges uniformly to $x_{0}$ as $k \rightarrow \infty$.

Proof. Let us prove item (i). Since the sequence of functions $\left\{x_{k}\right\}$ converges uniformly to $x_{0}$, it follows from Moore-Osgood Theorem that $x_{k}\left(t^{-}\right) \rightarrow x_{0}\left(t^{-}\right)$, for $t \in(a, b]$ and $x_{k}\left(t^{+}\right) \rightarrow x_{0}\left(t^{+}\right)$, for $t \in[a, b)$.

Now, let us prove item (ii). Note that it is enough to prove that the set $\left\{x_{k}:[a, b] \rightarrow\right.$ $X, k \in \mathbb{N}\}$ is equiregulated, since by Lemma 1.14, it follows that $x_{k}$ converges uniformly to $x_{0}$.

Since $x_{0}$ is a regulated function, its lateral limits exist.

Let $t_{0} \in[a, b]$ and $\varepsilon>0$ be given. Then there is $\delta>0$ such that, for every $t \in[a, b]$, we have

- if $t_{0}-\delta \leq t<t_{0}$, then $\left\|x_{0}\left(t_{0}^{-}\right)-x_{0}(t)\right\|<\varepsilon$,

- if $t_{0}<t \leq t_{0}+\delta$, then $\left\|x_{0}(t)-x_{0}\left(t_{0}^{+}\right)\right\|<\varepsilon$.

By hypotheses, there is an integer $n_{0}$ such that for every $n \geq n_{0}$, we get the following inequalities:

$$
\begin{gathered}
\left\|x_{n}\left(t_{0}-\delta\right)-x_{0}\left(t_{0}-\delta\right)\right\|<\varepsilon, \quad\left\|x_{n}\left(t_{0}\right)-x_{0}\left(t_{0}\right)\right\|<\varepsilon, \quad\left\|x_{n}\left(t_{0}^{+}\right)-x_{0}\left(t_{0}^{+}\right)\right\|<\varepsilon \\
\left\|x_{n}\left(t_{0}+\delta\right)-x_{0}\left(t_{0}+\delta\right)\right\|<\varepsilon \quad \text { and }\left\|x_{n}\left(t_{0}^{-}\right)-x_{0}\left(t_{0}^{-}\right)\right\|<\varepsilon
\end{gathered}
$$

If $t \in[a, b]$ is such that $t_{0}-\delta \leq t<t_{0}$, then, for every $n \geq n_{0}$, we have

$$
\begin{aligned}
0 \leq\left\|x_{n}\left(t_{0}^{-}\right)-x_{n}(t)\right\| & \leq\left\|x_{n}\left(t_{0}^{-}\right)-x_{0}\left(t_{0}^{-}\right)\right\|+\left\|x_{0}\left(t_{0}^{-}\right)-x_{0}(t)\right\| \\
& +\left\|x_{0}(t)-x_{n}(t)\right\|<\varepsilon+\varepsilon+\varepsilon=3 \varepsilon .
\end{aligned}
$$

If $t \in[a, b]$ is such that $t_{0}<t \leq t_{0}+\delta$, then, for every $n \geq n_{0}$, we obtain

$$
\begin{aligned}
0 \leq\left\|x_{n}(t)-x_{n}\left(t_{0}^{+}\right)\right\| & \leq\left\|x_{n}(t)-x_{0}(t)\right\|+\left\|x_{0}(t)-x_{0}\left(t_{0}^{+}\right)\right\| \\
& +\left\|x_{n}\left(t_{0}^{+}\right)-x_{0}\left(t_{0}^{+}\right)\right\|<\varepsilon+\varepsilon+\varepsilon=3 \varepsilon
\end{aligned}
$$

which implies that the sequence $\left\{x_{n}\right\}$ is equiregulated and we have the desired result. 
The next lemma is also a new result and it guarantees that, if a sequence of functions $\left\{x_{k}\right\}_{k=1}^{\infty}$ is bounded by an equiregulated sequence of functions, then $\left\{x_{k}\right\}_{k=1}^{\infty}$ is also equiregulated.

Lemma 1.16. Let $\left\{x_{k}\right\}_{k=1}^{\infty}$ be a sequence of functions from $[a, b]$ to $X$ and $\left\{h_{k}\right\}_{k=1}^{\infty}$ be an equiregulated sequence of functions from $[a, b]$ to $\mathbb{R}$. Suppose, for each $k=1,2, \ldots$, the function $x_{k}$ satisfies

$$
\left\|x_{k}\left(s_{2}\right)-x_{k}\left(s_{1}\right)\right\| \leq\left|h_{k}\left(s_{2}\right)-h_{k}\left(s_{1}\right)\right|
$$

for every $s_{1}, s_{2} \in[a, b]$. Then the sequence $\left\{x_{k}\right\}_{k=1}^{\infty}$ is equiregulated.

Proof. Let $\varepsilon>0$ be given. Since the sequence $\left\{h_{k}\right\}_{k=1}^{\infty}$ is equiregulated, it follows from Theorem 1.2 that there is a $\delta$-fine partition $a=t_{0}<t_{1}<\ldots<t_{m}=b$ such that

$$
\left|h_{k}\left(t^{\prime}\right)-h_{k}(t)\right| \leq \varepsilon
$$

for every $k \in \mathbb{N}$ and $\left[t, t^{\prime}\right] \subset\left(t_{j-1}, t_{j}\right)$, for $j=1,2, \ldots, m$. Using (1.2.1), there is a $\delta$-fine partition $a=t_{0}<t_{1}<\ldots<t_{m}=b$ such that

$$
\left\|x_{k}\left(t^{\prime}\right)-x_{k}(t)\right\| \leq \varepsilon
$$

for every $k \in \mathbb{N}$ and $\left[t, t^{\prime}\right] \subset\left(t_{j-1}, t_{j}\right)$ for $j=1,2, \ldots, m$. Therefore Theorem 1.2 implies that the sequence $\left\{x_{k}\right\}_{k=1}^{\infty}$ is equiregulated.

The next result is an immediate consequence of Lemmas 1.14 and 1.16 and it will be essential to prove results concerning continuous dependence of solutions on parameters in Chapter 6. It is a new result which can be found in [32].

Corollary 1.17. Let $\left\{x_{k}\right\}_{k=1}^{\infty}$ be a sequence of functions from $[a, b]$ to $X$ and $\left\{h_{k}\right\}_{k=1}^{\infty}$ be an equiregulated sequence of functions from $[a, b]$ to $\mathbb{R}$. Suppose the function $x_{k}$ satisfies condition (1.2.1) for every $k=1,2, \ldots$ and $s_{1}, s_{2} \in[a, b]$. If the sequence $\left\{x_{k}\right\}_{k=1}^{\infty}$ converges pointwisely to a function $x_{0}$, then the sequence $\left\{x_{k}\right\}_{k=1}^{\infty}$ converges uniformly to $x_{0}$. 


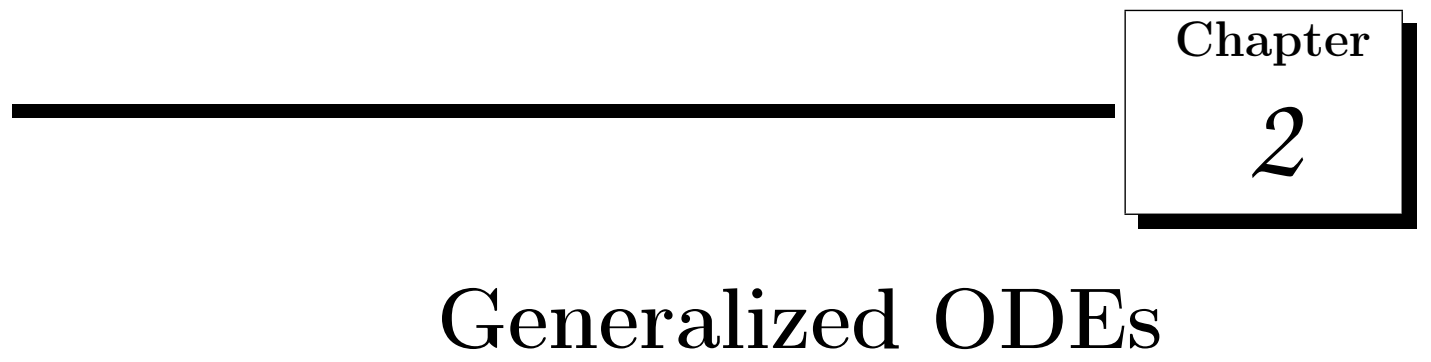

This chapter is divided in two sections. The first section is devoted to presenting basic concepts and main properties about the Kurzweil integral. In the second section, we present the basic theory about generalized ordinary differential equations (generalized ODEs, for short), which appear in the context of the Kurzweil integral. The main references for this chapter are [72], [73] and [94].

\subsection{The Kurzweil integral}

We start this section by introducing some basic concepts concerning the Kurzweil integral.

Let $-\infty<a<b<+\infty$ be given and consider the interval $[a, b] \subset \mathbb{R}$. Let $J$ be a compact interval such that $J \subset[a, b]$. Then any point-interval pair $(\tau, J)$ is called a tagged interval, where $\tau \in[a, b]$ is a $\operatorname{tag}$ of $J$.

A finite collection $\left\{\left(\tau_{j}, J_{j}\right), j=1,2, \ldots, k\right\}$ of tagged intervals is called a system in $[a, b]$ if $\tau_{j} \in J_{j} \subset[a, b]$, for every $j=1, \ldots, k$, and the intervals $J_{j}$ are nonoverlapping, that is, $\operatorname{Int}\left(J_{i}\right) \cap \operatorname{Int}\left(J_{j}\right)=\emptyset$, for $i \neq j$, where $\operatorname{Int}\left(J_{j}\right)$ denotes the interior of an interval $J_{j}$.

A system $\left\{\left(\tau_{j}, J_{j}\right), j=1,2, \ldots, k\right\}$ is called a tagged partition of $[a, b]$, whenever

$$
\bigcup_{j=1}^{k} J_{j}=[a, b] .
$$


We say that a positive function $\delta:[a, b] \rightarrow(0,+\infty)$ is a gauge on $[a, b]$ and a tagged interval $(\tau, J)$ with $\tau \in[a, b]$ is $\delta$-fine, if

$$
J \subset(\tau-\delta(\tau), \tau+\delta(\tau))
$$

We say that a tagged partition $\left\{\left(\tau_{j}, J_{j}\right), j=1, \ldots, k\right\}$ is $\delta$-fine, if the point-interval pair $\left(\tau_{j}, J_{j}\right)$ is $\delta$-fine, for every $j=1, \ldots, k$.

Using these concepts and terminology, we are able to present the concept of Kurzweil integrable function.

Let $X$ be a Banach space with norm $\|\cdot\|$.

Definition 2.1. A function $U:[a, b] \times[a, b] \rightarrow X$ is called Kurzweil integrable on $[a, b]$, if there is an element $I \in X$ such that given $\varepsilon>0$, there is a gauge $\delta$ on $[a, b]$ satisfying

$$
\left\|\sum_{i=1}^{k}\left[U\left(\tau_{i}, s_{i}\right)-U\left(\tau_{i}, s_{i-1}\right)\right]-I\right\|<\varepsilon,
$$

for every $\delta$-fine tagged partition of $[a, b]$. In this case, $I$ is called the Kurzweil integral of $U$ over $[a, b]$ and it will be denoted by $\int_{a}^{b} D U(\tau, t)$.

Alternatively, we denote the expression $\sum_{i=1}^{k}\left[U\left(\tau_{i}, s_{i}\right)-U\left(\tau_{i}, s_{i-1}\right)\right]$ by $S(U, D)$, where $D$ represents the chosen tagged partition, that is, $D=\left\{\left(\tau_{i},\left[s_{i-1}, s_{i}\right]\right), i=1,2, \ldots, k\right\}$.

The definition above generalizes the well-known Kurzweil-Henstock integral of a function $f:[a, b] \rightarrow \mathbb{R}^{n}$, which is obtained by setting $U(\tau, t)=f(\tau) t$. The Kurzweil-HenstockStieltjes integral of a function $f:[a, b] \rightarrow \mathbb{R}^{n}$ with respect to a function $g:[a, b] \rightarrow \mathbb{R}$, which appears in the definition of a measure differential equation, corresponds to the choice $U(\tau, t)=f(\tau) g(t)$ and will be denoted by $\int_{a}^{b} f(s) \mathrm{d} g(s)$.

We point out that it is possible to extend the concept of Kurzweil-Henstock-Stieltjes integral to unbounded intervals given by $(-\infty, c]$ or even $[c,+\infty)$. Let $\delta(-\infty)$ and $\delta(\infty)$ be positives numbers. We consider the $\delta$-neighborhoods of $-\infty$ and of $+\infty$ as follows

$$
[-\infty,-1 / \delta(-\infty)) \text { and }(1 / \delta(\infty), \infty]
$$

Then, $-\infty$ and $\infty$ are necessarily the tags of these $\delta$-neighborhoods with respect to any given gauge defined on the extended real line $\overline{\mathbb{R}}$. 
Then, in order to obtain a definition for the following integral

$$
\int_{-\infty}^{+\infty} f(t) d g(t)
$$

we consider $f(-\infty)=f(\infty)=0$, which implies that given a $\delta$-fine tagged partition of $\overline{\mathbb{R}}$, we obtain from the Riemann sum

$$
\sum_{i=1}^{k(d)} f\left(\tau_{i}\right)\left[g\left(t_{i}\right)-g\left(t_{i-1}\right)\right]
$$

that the first and last terms of the sum equal zero, since $\tau_{1}=t_{0}=-\infty$ and $\tau_{k(d)}=+\infty$. Here, we use the convention $0 \cdot \infty=0$. For other details, the reader may want to consult [65].

It is important noticing that Definition 2.1 makes sense only if, for a given gauge $\delta$ of $[a, b]$, there exists at least one $\delta$-fine tagged partition $D$ of $[a, b]$. This is the content of the next result and a proof can be found in [60], Theorem 4.1.

Lemma 2.2 (Cousin Lemma). Given a gauge $\delta$ on $[a, b]$, there is a $\delta$-fine tagged partition $D$ of $[a, b]$.

Similarly to Riemann and Lebesgue integrals, the Kurzweil integral has the usual properties of linearity, additivity with respect to adjacent intervals, integrability on subintervals, among others.

The next result is known as Saks-Henstock Lemma. It receives this name, since it was formulated first by the mathematician S. Saks and later, this result was reformulated by R. Henstock for generalized integrals using Riemann-sums.

Lemma 2.3 (Saks-Henstock - [94], Lemma 1.13). Let $U:[a, b] \times[a, b] \rightarrow X$ be Kurzweil integrable on $[a, b]$. Given $\varepsilon>0$, let $\delta$ be a gauge on $[a, b]$ such that

$$
\left\|\sum_{j=1}^{k}\left[U\left(\tau_{j}, \alpha_{j}\right)-U\left(\tau_{j}, \alpha_{j-1}\right)\right]-\int_{a}^{b} D U(\tau, t)\right\|<\varepsilon,
$$

for every $\delta$-fine tagged partition $D=\left\{\left(\tau_{j},\left[\alpha_{j-1}, \alpha_{j}\right]\right), j=1,2, \ldots, k\right\}$ of $[a, b]$. If

$$
a \leq \beta_{1} \leq \xi_{1} \leq \gamma_{1} \leq \beta_{2} \leq \xi_{2} \leq \gamma_{2} \leq \ldots \leq \beta_{m} \leq \xi_{m} \leq \gamma_{m} \leq b
$$


represents a $\delta$-fine system $\left\{\left(\xi_{j},\left[\beta_{j}, \gamma_{j}\right]\right), j=1,2, \ldots, m\right\}$, that is,

$$
\xi_{j} \in\left[\beta_{j}, \gamma_{j}\right] \subset\left(\xi_{j}-\delta\left(\xi_{j}\right), \xi_{j}+\delta\left(\xi_{j}\right)\right), \quad j=1,2, \ldots, m
$$

then

$$
\left\|\sum_{j=1}^{m}\left[U\left(\xi_{j}, \beta_{j}\right)-U\left(\xi_{j}, \gamma_{j}\right)-\int_{\beta_{j}}^{\gamma_{j}} D U(\tau, t)\right]\right\|<\varepsilon .
$$

The next Hake-type theorem concerns the Cauchy extension for the Kurzweil integral. It is a generalization of Theorem 1.14 from [94] for Banach space-valued functions. The proof follows analogously to the $\mathbb{R}^{n}$-valued case.

Theorem 2.4 (Cauchy Extension - [94], Theorem 1.14). If $U:[a, b] \times[a, b] \rightarrow X$ is $a$ function such that for every $c \in[a, b), U$ is Kurzweil integrable on $[a, c]$ and the limit

$$
\lim _{c \rightarrow b^{-}}\left[\int_{a}^{c} D U(\tau, t)-U(b, c)+U(b, b)\right]=I \in X
$$

exists, then the function $U$ is Kurzweil integrable on $[a, b]$ and it satisfies

$$
\int_{a}^{b} D U(\tau, t)=I
$$

Similarly, if the function $U$ is Kurzweil integrable on $[c, b]$ for every $c \in(a, b]$ and the limit

$$
\lim _{c \rightarrow a^{+}}\left[\int_{c}^{b} D U(\tau, t)+U(a, c)-U(a, a)\right]=I \in X
$$

exists, then the function $U$ is Kurzweil integrable on $[a, b]$ and we have

$$
\int_{a}^{b} D U(\tau, t)=I
$$

The following Hake-type theorem for the Kurzweil-Henstock-Stieltjes integral is a special case of Theorem 2.4. This result will be essential to prove our main results.

Corollary 2.5. Consider a pair of functions $f:[a, b] \rightarrow \mathbb{R}^{n}$ and $g:[a, b] \rightarrow \mathbb{R}$.

(i) Assume that the Kurzweil-Henstock-Stieltjes integral $\int_{a}^{t} f(s) \mathrm{d} g(s)$ exists for every $t \in[a, b)$ and

$$
\lim _{t \rightarrow b^{-}}\left(\int_{a}^{t} f(s) \mathrm{d} g(s)+f(b)(g(b)-g(t))\right)=I .
$$

Then $\int_{a}^{b} f(s) \mathrm{d} g(s)=I$. 
(ii) Assume that the Kurzweil-Henstock-Stieltjes integral $\int_{t}^{b} f(s) \mathrm{d} g(s)$ exists for every $t \in(a, b]$ and

$$
\lim _{t \rightarrow a^{+}}\left(\int_{t}^{b} f(s) \mathrm{d} g(s)+f(a)(g(t)-g(a))\right)=I .
$$

Then $\int_{a}^{b} f(s) \mathrm{d} g(s)=I$.

Proof. We will prove only item (i), since item (ii) follows analogously. Define the function $U:[a, b] \times[a, b] \rightarrow X$ by

$$
U(\tau, t)=f(\tau) g(t)
$$

Since the integral $\int_{a}^{t} f(s) \mathrm{d} g(s)$ exists for every $t \in[a, b)$, then $U$ is Kurzweil integrable over $[a, t]$ for every $t \in[a, b)$. Also, since

$$
\lim _{t \rightarrow b^{-}}\left(\int_{a}^{t} f(s) \mathrm{d} g(s)+f(b)[g(b)-g(t)]\right)=I,
$$

then, from the definition of $U$, we obtain

$$
\lim _{t \rightarrow b^{-}}\left[\int_{a}^{t} D U(\tau, s)-U(b, t)+U(b, b)\right]=I .
$$

Thus all hypotheses of Theorem 2.4 are satisfied and we have

$$
\int_{a}^{b} D U(\tau, t)=I
$$

which implies the desired result.

If $U:[a, b] \times[a, b] \rightarrow X$ is Kurzweil integrable, it is not always true that its indefinite integral $\int_{a}^{s} D U(\tau, t), s \in[a, b]$, is continuous. The next theorem describes the behavior of its indefinite integral.

Theorem 2.6 ([94], Theorem 1.16). Let $U:[a, b] \times[a, b] \rightarrow X$ be Kurzweil integrable over $[a, b]$ and let $c \in[a, b]$. Then,

$$
\lim _{s \rightarrow c}\left[\int_{a}^{s} D U(\tau, t)-U(c, s)+U(c, c)\right]=\int_{a}^{c} D U(\tau, t) .
$$

and

$$
\lim _{s \rightarrow c}\left[\int_{s}^{b} D U(\tau, t)-U(c, s)+U(c, c)\right]=\int_{c}^{b} D U(\tau, t) .
$$


The next result is a special case of Theorem 2.6 for the Kurzweil-Henstock-Stieltjes integral. It will be essential to prove our main results.

Corollary 2.7. Let $f:[a, b] \rightarrow \mathbb{R}^{n}$ and $g:[a, b] \rightarrow \mathbb{R}$ be a pair of functions such that $g$ is regulated and the Kurzweil-Henstock-Stieltjes integral $\int_{a}^{b} f(s) \mathrm{d} g(s)$ exists. Then the functions

$$
h(t)=\int_{a}^{t} f(u) \mathrm{d} g(u) \text { and } k(t)=\int_{t}^{b} f(u) \mathrm{d} g(u), \quad t \in[a, b]
$$

are regulated on $[a, b]$ and satisfy

$$
\begin{aligned}
h\left(t^{+}\right)=h(t)+f(t) \Delta^{+} g(t), & t \in[a, b), \\
h\left(t^{-}\right)=h(t)-f(t) \Delta^{-} g(t), & t \in(a, b], \\
k\left(t^{+}\right) & =k(t)-f(t) \Delta^{+} g(t), \quad t \in[a, b), \\
k\left(t^{-}\right) & =k(t)+f(t) \Delta^{-} g(t), \quad t \in(a, b] .
\end{aligned}
$$

Proof. Using the fact that the integral $\int_{a}^{b} f(u) \mathrm{d} g(u)$ exists, we have, by Corollary 2.5, for $t \in[a, b)$

$$
\lim _{s \rightarrow t^{+}}\left[\int_{a}^{s} f(u) \mathrm{d} g(u)-f(t) g(s)+f(t) g(t)\right]=\int_{a}^{t} f(u) \mathrm{d} g(u)
$$

that is,

$$
\lim _{s \rightarrow t^{+}}\left[\int_{a}^{s} f(u) \mathrm{d} g(u)\right]=\int_{a}^{t} f(u) \mathrm{d} g(u)+f(t)\left(g\left(t^{+}\right)-g(t)\right) .
$$

Therefore, for $t \in[a, b)$, we obtain

$$
h\left(t^{+}\right)=h(t)+f(t) \Delta^{+} g(t) .
$$

To prove the other equalities, we just have to apply similar arguments.

We recall that a function $f:[a, b] \rightarrow X$ is a finite step function, if there is a finite division $a=\beta_{0}<\beta_{1}<\ldots<\beta_{m}=b$ such that, in every open interval $\left(\beta_{i-1}, \beta_{i}\right)$, $i=1,2, \ldots, m$, the function $f$ is identically equal to an element $c_{i} \in X$.

Remark 2.8. If $f:[a, b] \rightarrow \mathbb{R}^{n}$ is a finite step function and $g:[a, b] \rightarrow \mathbb{R}$ is of bounded variation, then the Kurzweil-Henstock-Stieltjes integral $\int_{a}^{b} f(s) \mathrm{d} g(s)$ exists and it is possible to calculate it by the formula

$$
\int_{a}^{b} f(s) \mathrm{d} g(s)=f(a)\left[g\left(a^{+}\right)-g(a)\right]+\sum_{j=1}^{m} f\left(\beta_{j}\right)\left[g\left(\beta_{j}^{+}\right)-g\left(\beta_{j}^{-}\right)\right]+
$$




$$
+f(b)\left[g(b)-g\left(b^{-}\right)\right]+\sum_{j=1}^{m} c_{j}\left[g\left(\beta_{j}^{-}\right)-g\left(\beta_{j-1}^{+}\right)\right] .
$$

For a proof of this result, see [94], Proposition 1.33.

As a consequence of this result, we can obtain a more general result concerning the existence of the Kurzweil-Henstock-Stieltjes integral $\int_{a}^{b} f(s) \mathrm{d} g(s)$. This result can be found in [94], Corollary 1.34.

Theorem 2.9. If $f:[a, b] \rightarrow \mathbb{R}^{n}$ is a regulated function on $[a, b]$ and $g:[a, b] \rightarrow \mathbb{R}$ is a nondecreasing function, then the Kurzweil-Henstock-Stieltjes integral $\int_{a}^{b} f(s) \mathrm{d} g(s)$ exists.

We finish this section by mentioning an important inequality for the Kurzweil-HenstockStieltjes integral. It is a Gronwall-type inequality and it will be essential to prove our main results. For a proof of this result, the reader may want to consult [94], Corollary 1.43 .

Theorem 2.10. Let $h:[a, b] \rightarrow[0, \infty)$ be a nondecreasing left-continuous function, $k>0$ and $l \geq 0$. Assume that $\psi:[a, b] \rightarrow[0, \infty)$ is bounded and satisfies

$$
\psi(\xi) \leq k+l \int_{a}^{\xi} \psi(\tau) \mathrm{d} h(\tau), \quad \xi \in[a, b] .
$$

Then $\psi(\xi) \leq k e^{l(h(\xi)-h(a))}$ for every $\xi \in[a, b]$.

This result remains true if we replace the left-continuity of the function $h$ by its rightcontinuity. In this case, we obtain

$$
\psi(\xi) \leq k e^{l(h(b)-h(\xi))}, \quad \xi \in[a, b]
$$

For a proof of this result, see [94], Corollary 1.44.

After having presented the basis of the Kurzweil integration theory and the particularities of the Kurzweil-Henstock-Stieltjes integral, we no longer mention explicitly that $\int_{a}^{b} D U(\tau, t)$ stands for the Kurzweil integral and that $\int_{a}^{b} f(s) \mathrm{d} g(s)$ stands for the KurzweilHenstock-Stieltjes integral, because we understand that the reader will not be confused by them. 


\subsection{Generalized ODEs}

In this section, we introduce the basic concepts concerning generalized ordinary differential equations (generalized ODEs, for short). A basic source of the theory of generalized ordinary differential equations is the book [94] and it is the main reference used here.

Consider a set $O \subset X$, an interval $I \subset \mathbb{R}$ and a function $G: O \times I \rightarrow X$. We say that $x: I \rightarrow O$ is a solution of the generalized ordinary differential equation

$$
\frac{\mathrm{d} x}{\mathrm{~d} \tau}=D G(x, t)
$$

on the interval $I$, whenever

$$
x(b)-x(a)=\int_{a}^{b} D G(x(\tau), t),
$$

for every $a, b \in I$.

It is important noticing that the notation used in equation (2.2.1) is only symbolical. The letter $D$ indicates that (2.2.1) is a generalized ordinary differential equation, this concept being defined via its solutions. Even the notation $\frac{d x}{d \tau}$ does not mean that the solution has a derivative.

Without loss of generality, we can always assume that the right-hand side of a generalized ODE satisfies $G(x, 0)=0$ for every $x \in O$. Otherwise, we let

$$
\tilde{G}(x, t)=G(x, t)-G(x, 0), \quad x \in O \text { and } t \in I,
$$

and consider the equation

$$
\frac{\mathrm{d} x}{\mathrm{~d} \tau}=D \tilde{G}(x, t)
$$

Then, we have $\tilde{G}(x, 0)=0$, for every $x \in O$, and it follows directly from the definition of the Kurzweil integral that this new equation has the same set of solutions as the original one.

In order to obtain reasonable results for generalized ODEs, we will restrict ourselves to the following class of functions $G: O \times I \rightarrow X$.

Definition 2.11. Let $O \subset X, I \subset \mathbb{R}$ an interval of the real line, $\Omega=O \times I$. Assume that $h: I \rightarrow \mathbb{R}$ is a nondecreasing function. We say that a function $G: \Omega \rightarrow X$ belongs 
to the class $\mathcal{F}(\Omega, h)$, if it satisfies the inequalities

$$
\left\|G\left(x, s_{2}\right)-G\left(x, s_{1}\right)\right\| \leq\left|h\left(s_{2}\right)-h\left(s_{1}\right)\right|
$$

for every $x \in O$ and every $s_{1}, s_{2} \in I$, and

$$
\left\|G\left(x, s_{2}\right)-G\left(x, s_{1}\right)-G\left(y, s_{2}\right)+G\left(y, s_{1}\right)\right\| \leq\|x-y\| \cdot\left|h\left(s_{2}\right)-h\left(s_{1}\right)\right|,
$$

for every $x, y \in O$ and every $s_{1}, s_{2} \in I$.

The next result describes the integral equation fulfilled by a solution of generalized ordinary differential equation whenever it exists. For a proof of this result, see [94].

Proposition 2.12 ([94], Proposition 3.5). Let $x:[a, b] \rightarrow X$ be a solution of generalized ordinary differential equation

$$
\frac{d x}{d \tau}=D G(x(\tau), t)
$$

on $[a, b]$, then for every fixed $\gamma \in[a, b]$, we have

$$
x(s)=x(\gamma)+\int_{\gamma}^{s} D G(x(\tau), t), \quad s \in[a, b]
$$

Reciprocally, if $x:[a, b] \rightarrow X$ satisfies the integral equation (2.2.6) for any $\gamma \in[a, b]$, then $x$ is a solution of generalized ordinary differential equation (2.2.5).

The next result describes the solutions of (2.2.5) depending on the function $G: \Omega \rightarrow$ $X$. In particular, if the function $G$ is continuous with respect to the second variable, then any solution $x:[a, b] \rightarrow X$ of the generalized ODE (2.2.5) is a continuous function.

Proposition 2.13 ([94], Proposition 3.6). Let $x:[a, b] \rightarrow X$ be a solution of generalized ordinary differential equation $(2.2 .5)$ on $[a, b]$. Then

$$
\lim _{s \rightarrow \sigma}[x(s)-G(x(\sigma), s)+G(x(\sigma), \sigma)]=x(\sigma),
$$

for every $\sigma \in[a, b]$.

The following lemma combines two statements from [94] (see Lemma 3.9 and Corollary 3.16) for Banach space-valued functions. It is an important result.

Lemma 2.14. Let $O \subset X, \Omega=O \times[a, b]$. Assume that $G: \Omega \rightarrow X$ belongs to the class $\mathcal{F}(\Omega, h)$. If $x:[a, b] \rightarrow O$ is a regulated function, then the integral $\int_{a}^{b} D G(x(\tau), t)$ exists 
and

$$
\left\|\int_{a}^{b} D G(x(\tau), t)\right\| \leq h(b)-h(a) .
$$

The next lemma guarantees that the solutions of generalized ordinary differential equation (2.2.5) are of bounded variation, whenever the function $G$ satisfies condition (2.2.3). It combines Lemma 3.10 and Corollary 3.11, both from [94]

Lemma 2.15. Suppose $G: \Omega \rightarrow X$ satisfies condition (2.2.3). If $[\alpha, \beta] \subset[a, b]$ and $x:[\alpha, \beta] \rightarrow X$ is a solution of (2.2.5), then the inequality

$$
\left\|x\left(s_{1}\right)-x\left(s_{2}\right)\right\| \leq\left|h\left(s_{2}\right)-h\left(s_{1}\right)\right|
$$

holds for every $s_{1}, s_{2} \in[\alpha, \beta]$. Also, $x$ is of bounded variation on $[\alpha, \beta]$ and

$$
\operatorname{var}_{\alpha}^{\beta} x \leq h(\beta)-h(\alpha)<+\infty
$$

Moreover, if $h$ is continuous at $t \in[\alpha, \beta]$, then $t$ is a point of continuity of the solution $x:[\alpha, \beta] \rightarrow X$.

Finally, we present the last result of this chapter. It describes the discontinuities of the solutions of a generalized ordinary differential equation given by (2.2.5), whenever the function $G$ satisfies condition (2.2.3). It describes the "jumps" of the solutions. This result can be found in [94], Lemma 3.12. For a proof of this result in a Banach space setting, the reader may consult [48].

Lemma 2.16. If $x:[a, b] \rightarrow X$ is a solution of (2.2.5) and $G: \Omega \rightarrow X$ satisfies condition $(2.2 .3)$, then

$$
x\left(s^{+}\right)-x(s)=\lim _{\sigma \rightarrow s^{+}} x(\sigma)-x(s)=G\left(x(s), s^{+}\right)-G(x(s), s)
$$

for $s \in[a, b)$ and

$$
x(s)-x\left(s^{-}\right)=x(s)-\lim _{\sigma \rightarrow s^{-}} x(\sigma)=G(x(s), s)-G\left(x(s), s^{-}\right)
$$

for $s \in(a, b]$ where $G\left(x, s^{+}\right)=\lim _{\sigma \rightarrow s^{+}} G(x, \sigma)$ for $s \in[a, b)$ and $G\left(x, s^{-}\right)=\lim _{\sigma \rightarrow s^{-}} G(x, \sigma)$ for $s \in(a, b]$. 


\section{- \\ Dynamic equations on time scales}

The theory of time scales is a recent theory which started to be developed by Stefan Hilger on his doctor thesis (see [62]) in order to unify the discrete and continuous analysis. When we are dealing with the equations provided by this theory, the idea is to obtain a result for a dynamic equation where the domain of the unknown function is a time scale. A time scale is a closed nonempty subset of the real numbers. For instance, if the time scale is taken as the set of the real numbers, we have the continuous case and, hence, results for ordinary differential equations. On the other hand, if the time scale is taken as the set of the integer numbers, we are in the discrete case and, hence, the results can be applied to difference equations. Also, we can obtain several results depending on the chosen time scale.

In the present chapter, our goal is to introduce the basic concepts concerning this theory and present some fundamental results found in the literature. This chapter is divided in four sections. In the first one, we introduce the basic concepts concerning this theory. The second section is devoted to the basic concepts and properties of deltaderivatives and delta-differentiable functions. The third section presents Riemann deltaintegrals and their properties. Finally, the last section is devoted to the basic concepts on Kurzweil-Henstock delta-integrals and their properties.

The basis of the theory of time scales can be found in the books [17] and [18] and these references are used here in order to develop the Sections 4.1, 4.2 and 4.3. For the last section, namely Section 4.4, main reference is [90]. 


\subsection{Time scales calculus}

A time scale is a closed nonempty subset of the real numbers. Therefore the sets

$$
[a, b], \quad \mathbb{Z}, \quad \mathbb{N}, \quad \mathbb{R}, \quad \text { Cantor set }
$$

are examples of time scales, whereas open intervals, half-open intervals and $\mathbb{Q}$ are not examples of time scales, since these sets are not closed in $\mathbb{R}$. We denote a time scale by $\mathbb{T}$.

Let $\mathbb{T}$ be a time scale. We assume that a time scale $\mathbb{T}$ has the topology that it inherits from the real numbers with the standard topology.

For $t \in \mathbb{T}$, we define the forward jump operator $\sigma: \mathbb{T} \rightarrow \mathbb{T}$ by

$$
\sigma(t)=\inf \{s \in \mathbb{T} ; s>t\}
$$

and the backward jump operator $\rho: \mathbb{T} \rightarrow \mathbb{T}$ by

$$
\rho(t)=\sup \{s \in \mathbb{T} ; s<t\}
$$

In the above definitions, we take $\inf \emptyset=\sup \mathbb{T}$ and $\sup \emptyset=\inf \mathbb{T}$, which imply that $\sigma(t)=t$, if $\mathbb{T}$ has a maximum $t$ and $\rho(t)=t$, if $\mathbb{T}$ has a minimum $t$.

We say that $t \in \mathbb{T}$ is a right-scattered point, if $\sigma(t)>t$, and $t \in \mathbb{T}$ is a left-scattered point, if $\rho(t)<t$. If $t$ is right-scattered and left-scattered point at the same time, then $t$ is called an isolated point.

We say that $t \in \mathbb{T}$ is right-dense point, whenever $\sigma(t)=t$, and $t$ is called left-dense, if $\rho(t)=t$. If $t$ is right-dense and left-dense point at the same time, then $t$ is called a dense point.

In order to illustrate these concepts, we present some examples. All these examples can be found in [17].

Example 3.1. If $\mathbb{T}=\mathbb{R}$, then for $t \in \mathbb{R}$, we obtain

$$
\begin{gathered}
\sigma(t)=\inf \{s \in \mathbb{R} ; s>t\}=\inf (t,+\infty)=t, \\
\rho(t)=\sup \{s \in \mathbb{R} ; s<t\}=\sup (-\infty, t)=t .
\end{gathered}
$$


Thus $t$ is a right-dense and left-dense point and, hence, $t$ is a dense point.

Example 3.2. If $\mathbb{T}=\mathbb{Z}$, then for any $t \in \mathbb{Z}$, we have

$$
\begin{gathered}
\sigma(t)=\inf \{s \in \mathbb{Z}: s>t\}=\inf \{t+1, t+2, t+3, \ldots\}=t+1>t \\
\rho(t)=\sup \{s \in \mathbb{Z}: s<t\}=\sup \{t-1, t-2, t-3, \ldots\}=t-1<t .
\end{gathered}
$$

Thus $t$ is a right-scattered and left-scattered point and, hence, $t$ is an isolated point.

Example 3.3. If $\mathbb{T}=q^{\mathbb{Z}} \cup\{0\}, q>1$, we get

$$
\begin{gathered}
\sigma(t)=\inf \{s \in \mathbb{T}: s>t\}=\inf \left\{q t, q^{2} t, q^{3} t, \ldots\right\}=q t>t, \text { for } t \neq 0, \\
\rho(t)=\sup \{s \in \mathbb{T}: s<t\}=\sup \left\{t / q, t / q^{2}, t / q^{3}, \ldots\right\}=t / q<t, \text { for } t \neq 0 .
\end{gathered}
$$

Therefore, $t \neq 0$ is right-scattered and left-scattered at the same time. On the other hand, if $t=0$, it follows that $t$ is right-dense.

In the sequel, we define the graininess function $\mu: \mathbb{T} \rightarrow[0, \infty)$ by

$$
\mu(t):=\sigma(t)-t
$$

By Examples 3.1, 3.2 and 3.3, it is clear that

- If $\mathbb{T}=\mathbb{R}$, then $\mu(t)=0$, for all $t \in \mathbb{T}$;

- If $\mathbb{T}=\mathbb{Z}$, then $\mu(t)=1$, for all $t \in \mathbb{T}$;

- If $\mathbb{T}=q^{\mathbb{Z}} \cup\{0\}$, then $\mu(t)=(q-1) t$, for every $t \neq 0 \in \mathbb{T}$ and $\mu(0)=0$.

In what follows, we present two more examples concerning backward and forward jump operators and graininess function. These examples can be found in [17].

Example 3.4. Let $a, b>0$ and consider the time scale:

$$
\mathbb{P}_{a, b}=\bigcup_{k=0}^{\infty}[k(a+b), k(a+b)+a]
$$


It is clear that

$$
\sigma(t)=\left\{\begin{array}{l}
t, \quad \text { if } \quad t \in \bigcup_{k=0}^{\infty}[k(a+b), k(a+b)+a), \\
t+b, \quad \text { if } t \in \bigcup_{k=0}^{\infty}\{k(a+b)+a\}
\end{array}\right.
$$

Then, we have

$$
\mu(t)=\left\{\begin{array}{c}
0, \quad \text { if } \quad t \in \bigcup_{k=0}^{\infty}[k(a+b), k(a+b)+a), \\
b, \quad \text { if } \quad t \in \bigcup_{k=0}^{\infty}\{k(a+b)+a\}
\end{array}\right.
$$

using the fact that $\mu(t)=\sigma(t)-t$.

Example 3.5. Consider the time scale given by

$$
\mathbb{T}=\mathbb{N}_{0}^{2}=\left\{n^{2}: n \in \mathbb{N}_{0}\right\}
$$

Then, for each $n \in \mathbb{N}_{0}$, we obtain

$$
\sigma\left(n^{2}\right)=(n+1)^{2}
$$

and

$$
\mu\left(n^{2}\right)=\sigma\left(n^{2}\right)-n^{2}=(n+1)^{2}-n^{2}=n^{2}+2 n+1-n^{2}=2 n+1 .
$$

By the definition of forward jump operator, we have

$$
\sigma(t)=\inf \left\{n^{2}: n^{2}>t\right\}
$$

Also, since $t \in \mathbb{T}$, then $t=m^{2}$, for $m \in \mathbb{N}_{0}$. As a consequence, we obtain

$$
\sigma(t)=(\sqrt{t}+1)^{2}
$$

and

$$
\mu(t)=(\sqrt{t}+1)^{2}-t=t+2 \sqrt{t}+1-t=2 \sqrt{t}+1,
$$

for every $t \in \mathbb{T}$.

Since any time scale $\mathbb{T}$ is a closed subset of the real numbers, it is clear from the definition that the values $\sigma(t)$ and $\rho(t)$ belong to $\mathbb{T}$, for every $t \in \mathbb{T}$. 
We define the set $\mathbb{T}^{k}$ which is derived from the time scale $\mathbb{T}$ as follows:

$$
\mathbb{T}^{k}= \begin{cases}\mathbb{T} \backslash(\rho(\sup \mathbb{T}), \sup \mathbb{T}], & \text { if } \quad \sup \mathbb{T}<\infty \\ \mathbb{T}, & \text { if } \quad \sup \mathbb{T}=\infty\end{cases}
$$

Let $a, b \in \mathbb{T}$ such that $a \leq b$. We denote and define an interval $[a, b]$ in $\mathbb{T}$ by

$$
[a, b]_{\mathbb{T}}=\{t \in \mathbb{T}: a \leq t \leq b\}
$$

Analogously, we define open intervals, half-open intervals, etc. If $b$ is a left-dense point, then $[a, b]_{\mathbb{T}^{k}}=[a, b]_{\mathbb{T}}$ and, if $b$ is a left-scattered point, then $[a, b]_{\mathbb{T}^{k}}=[a, b)_{\mathbb{T}}=[a, \rho(b)]_{\mathbb{T}}$.

\subsection{Differentiation}

In this section, we introduce the basic properties about delta derivative of a function $f: \mathbb{T} \rightarrow \mathbb{R}$.

Let $f: \mathbb{T} \rightarrow \mathbb{R}$ be a function. We define the so-called delta (or Hilger) derivative of $f$ at a point $t \in \mathbb{T}^{k}$ as follows.

Definition 3.6. Assume $f: \mathbb{T} \rightarrow \mathbb{R}$ is a function and let $t \in \mathbb{T}^{k}$. Then, we define $f^{\Delta}(t)$ to be the number (provided it exists) with the property that, given any $\varepsilon>0$, there is a neighborhood $U$ of $t$ (that is, $U=(t-\delta, t+\delta) \cap \mathbb{T}$ for some $\delta>0$ ) such that

$$
\left|[f(\sigma(t))-f(s)]-f^{\Delta}(t)[\sigma(t)-s]\right| \leq \varepsilon|\sigma(t)-s|, \quad \text { for every } \quad s \in U .
$$

We call $f^{\Delta}(t)$ the delta (or Hilger) derivative of $f$ at $t$. Moreover, we say that $f$ is delta (or Hilger) differentiable on $\mathbb{T}^{k}$, provided $f^{\Delta}(t)$ exists for all $t \in \mathbb{T}^{k}$. The function $f^{\Delta}: \mathbb{T}^{k} \rightarrow \mathbb{R}$ is called the delta-derivative of $f$ on $\mathbb{T}^{k}$.

In what follows, we present some examples to illustrate this definition. These examples can be found in [17].

Example 3.7. Let $f: \mathbb{T} \rightarrow \mathbb{R}$ be a function defined by

$$
f(t)=\alpha, \quad \text { for every } t \in \mathbb{T},
$$


where $\alpha \in \mathbb{R}$ is constant. Then $f^{\Delta} \equiv 0$. Indeed, for every $\varepsilon>0$, we have

$$
|f(\sigma(t))-f(s)-0 \cdot(\sigma(t)-s)|=|\alpha-\alpha-0|=0 \leq \varepsilon|\sigma(t)-s|,
$$

for all $s \in \mathbb{T}$.

Example 3.8. Let $f: \mathbb{T} \rightarrow \mathbb{R}$ be a function defined by

$$
f(t)=t, \quad \text { for every } t \in \mathbb{T} \text {. }
$$

Then $f^{\Delta} \equiv 1$. In fact, for every $\varepsilon>0$, we have

$$
|f(\sigma(t))-f(s)-1 \cdot(\sigma(t)-s)|=|\sigma(t)-s-(\sigma(t)-s)|=0 \leq \varepsilon|\sigma(t)-s|,
$$

for every $s \in \mathbb{T}$.

Example 3.9. Let $f: \mathbb{T} \rightarrow \mathbb{R}$ be a function defined by

$$
f(t)=t^{2}, \text { for all } t \in \mathbb{T} \text {. }
$$

Then $f^{\Delta}(t)=t+\sigma(t)$ for every $t \in \mathbb{T}^{k}$. This fact follows since for every $\varepsilon>0$, there exists a neighborhood $U$ of $t$, say, $U=(t-\delta, t+\delta) \cap \mathbb{T}$ for some $\delta>0$, such that

$$
\begin{gathered}
|f(\sigma(t))-f(s)-(t+\sigma(t)) \cdot(\sigma(t)-s)|=\left|\sigma(t)^{2}-s^{2}-(t-s+\sigma(t)+s) \cdot(\sigma(t)-s)\right| \leq \\
\leq\left|\sigma(t)^{2}-s^{2}-\left(\sigma(t)^{2}-s^{2}\right)-(t-s) \cdot(\sigma(t)-s)\right|= \\
\quad=|t-s| \cdot|\sigma(t)-s|<\delta|\sigma(t)-s| \leq \varepsilon|\sigma(t)-s|
\end{gathered}
$$

for every $s \in U$.

The next result describes some useful properties of delta derivatives. A proof of it can be found in [17], Theorem 1.16.

Theorem 3.10. Assume $f: \mathbb{T} \rightarrow \mathbb{R}$ is a function and let $t \in \mathbb{T}^{k}$. Then the following assertions hold:

(i) If $f$ is $\Delta$-differentiable at $t$, then $f$ is continuous at $t$. 
(ii) If $f$ is continuous at $t$ and $t$ is right-scattered, then $f$ is $\Delta$-differentiable at $t$ with

$$
f^{\Delta}(t)=\frac{f(\sigma(t))-f(t)}{\mu(t)}
$$

(iii) If $t$ is a right-dense point, then $f$ is $\Delta$-differentiable at $t$, if and only if, the limit

$$
\lim _{s \rightarrow t} \frac{f(t)-f(s)}{t-s}
$$

and is finite. In this case,

$$
f^{\Delta}(t)=\lim _{s \rightarrow t} \frac{f(t)-f(s)}{t-s}
$$

(iv) If $f$ is $\Delta$-differentiable at $t$, then

$$
f(\sigma(t))=f(t)+\mu(t) f^{\Delta}(t) .
$$

The next result describes the properties of $\Delta$-derivatives of sum, product and quotient of two functions. A proof of it can be found in [18], Theorem 1.20.

Theorem 3.11. Assume $f, g: \mathbb{T} \rightarrow \mathbb{R}$ are $\Delta$-differentiable at $t \in \mathbb{T}^{k}$. Then:

(i) The sum $f+g: \mathbb{T} \rightarrow \mathbb{R}$ is $\Delta$-differentiable at $t$ with

$$
(f+g)^{\Delta}(t)=f^{\Delta}(t)+g^{\Delta}(t)
$$

(ii) For any constant $\alpha, \alpha f: \mathbb{T} \rightarrow \mathbb{R}$ is $\Delta$-differentiable at $t$ with

$$
(\alpha f)^{\Delta}(t)=\alpha f^{\Delta}(t)
$$

(iii) The product $f g: \mathbb{T} \rightarrow \mathbb{R}$ is $\Delta$-differentiable at $t$ with

$$
(f g)^{\Delta}(t)=f^{\Delta}(t) g(t)+f(\sigma(t)) g^{\Delta}(t)=f(t) g^{\Delta}(t)+f^{\Delta}(t) g(\sigma(t)) .
$$


(iv) If $f(t) f(\sigma(t)) \neq 0$, then $1 / f$ is $\Delta$-differentiable at $t$ with

$$
\left(\frac{1}{f}\right)^{\Delta}(t)=\frac{-f^{\Delta}(t)}{f(t) f(\sigma(t))}
$$

(v) If $g(t) g(\sigma(t)) \neq 0$, then $f / g$ is $\Delta$-differentiable at $t$ and

$$
\left(\frac{f}{g}\right)^{\Delta}(t)=\frac{f^{\Delta}(t) g(t)-f(t) g^{\Delta}(t)}{g(t) g(\sigma(t))} .
$$

Now, we present an example which illustrates the properties above. Such example is borrowed from [17].

Example 3.12. Let $q>1$ and consider the following sets

$$
q^{\mathbb{Z}}:=\left\{q^{k}: k \in \mathbb{Z}\right\} \quad \text { and } \quad \overline{q^{\mathbb{Z}}}:=q^{\mathbb{Z}} \cup\{0\}
$$

Let $\mathbb{T}=\overline{q^{\mathbb{Z}}}$ and $f: \mathbb{T} \rightarrow \mathbb{R}$ be a function. As it was explained before, $t \in \mathbb{T} \backslash\{0\}$ is right-scattered. Then by the definition of delta-derivative, we have

$$
f^{\Delta}(t)=\frac{f(\sigma(t))-f(t)}{\mu(t)}=\frac{f(q t)-f(t)}{(q-1) t}, \text { for every } t \in \mathbb{T} \backslash\{0\}
$$

On the other hand, $t=0$ is a right-dense minimum. Therefore, when $t=0$, we have

$$
f^{\Delta}(0)=\lim _{s \rightarrow 0} \frac{f(\sigma(t))-f(s)}{\sigma(t)-s}=\lim _{s \rightarrow 0} \frac{f(0)-f(s)}{0-s}=\lim _{s \rightarrow 0} \frac{f(s)-f(0)}{s},
$$

provided the limit exists.

\subsection{Darboux and Riemann delta-integrals}

In this section, we present the basic concepts and properties about Darboux and Riemann delta-integrals. As before, we denote a time scale by $\mathbb{T}$ and, for $a, b \in \mathbb{T}$, we denote the time scale interval by $[a, b]_{\mathbb{T}}$.

We start this section by introducing two basic concepts which are fundamental to describe a class of functions which are Riemann $\Delta$-integrable.

Definition 3.13. A function $f: \mathbb{T} \rightarrow \mathbb{R}$ is called regulated, provided its right-sided limits exist (finite) at all right-dense points in $\mathbb{T}$ and its left-sided limits exist (finite) at all 
left-dense points in $\mathbb{T}$.

Definition 3.14. A function $f: \mathbb{T} \rightarrow \mathbb{R}$ is called $r d$-continuous (right-dense continuous), provided it is continuous at right-dense points in $\mathbb{T}$ and its left-sided limits exist (finite) at left-dense points in $\mathbb{T}$.

We denote the set of rd-continuous functions $f: \mathbb{T} \rightarrow \mathbb{R}$ by

$$
\mathcal{C}_{r d}=\mathcal{C}_{r d}(\mathbb{T})=\mathcal{C}_{r d}(\mathbb{T}, \mathbb{R})
$$

We point out that if $\mathbb{T}=\mathbb{R}$, the Definitions 3.13 and 3.14 coincide with the usual definitions of regulated and continuous functions respectively, since every point in $\mathbb{T}=\mathbb{R}$ is right-dense and left-dense at the same time.

We say that a partition $P$ of $[a, b]_{\mathbb{T}}$ is any finite ordered set

$$
P=\left\{t_{0}, t_{1}, \ldots, t_{n}\right\} \subset[a, b]_{\mathbb{T}}
$$

where $a=t_{0}<t_{1}<\ldots<t_{n}=b$. The number $n$ depends on the partition and we write $n=n(P)$. The set of all partitions of $[a, b]_{\mathbb{T}}$ will be denoted by $\mathcal{P}=\mathcal{P}(a, b)$.

Let $f$ be a real-valued bounded function on $[a, b]_{\mathbb{T}}$ and consider

$$
M=\sup \left\{f(t): t \in[a, b)_{\mathbb{T}}\right\} \quad \text { and } \quad m=\inf \left\{f(t): t \in[a, b)_{\mathbb{T}}\right\} .
$$

Define, for $1 \leq i \leq n$,

$$
M_{i}=\sup \left\{f(t): t \in\left[t_{i-1}, t_{i}\right)_{\mathbb{T}}\right\} \quad \text { and } \quad m_{i}=\inf \left\{f(t): t \in\left[t_{i-1}, t_{i}\right)_{\mathbb{T}}\right\}
$$

Using these concepts, we can define the upper Darboux $\Delta$-sum $U(f, P)$ and the lower Darboux $\Delta$-sum $L(f, P)$ of $f$ with respect to $P \in \mathcal{P}$ by

$$
U(f, P)=\sum_{i=1}^{n} M_{i}\left(t_{i}-t_{i-1}\right) \quad \text { and } \quad L(f, P)=\sum_{i=1}^{n} m_{i}\left(t_{i}-t_{i-1}\right),
$$

respectively. Also, by the definition, it follows

$$
U(f, P) \leq \sum_{i=1}^{n} M\left(t_{i}-t_{i-1}\right)=M(b-a)
$$


and

$$
L(f, P) \geq \sum_{i=1}^{n} m\left(t_{i}-t_{i-1}\right)=m(b-a)
$$

which implies

$$
m(b-a) \leq L(f, P) \leq U(f, P) \leq M(b-a)
$$

In this way, we define the upper and lower Darboux $\Delta$-integral of $f$ from $a$ to $b$ as follows

$$
U(f)=\inf \{U(f, P): P \in \mathcal{P}(a, b)\}
$$

and

$$
L(f)=\sup \{L(f, P): P \in \mathcal{P}(a, b)\},
$$

respectively. By (3.3.1) and the definition of upper and lower Darboux $\Delta$-integrals, it follows that $U(f)$ and $L(f)$ are finite real numbers.

We say that $f$ is $\Delta$-integrable (or delta-integrable) from $a$ to $b$ (or on $[a, b]_{\mathbb{T}}$ ), provided $L(f)=U(f)$, and we write $\int_{a}^{b} f(t) \Delta t$ for this value, in this case. We call this integral by Darboux $\Delta$-integral.

We denote by $\mathcal{P}_{\delta}=\mathcal{P}_{\delta}(a, b)$ the set of all $P \in \mathcal{P}(a, b)$ which have the following property: for each $i \in\{1,2, \ldots, n\}$ either

$$
t_{i}-t_{i-1} \leq \delta
$$

or

$$
t_{i}-t_{i-1}>\delta \quad \text { and } \quad \rho\left(t_{i}\right)=t_{i-1}
$$

where $a=t_{0}<t_{1}<\ldots<t_{n}=b$.

In what follows, we present the definitions of Riemann $\Delta$-sums and $\Delta$-integrals.

Definition 3.15. Let $f$ be a bounded function on $[a, b]_{\mathbb{T}}$ and let $P \in \mathcal{P}(a, b)$ be given by $a=t_{0}<t_{1}<\ldots<t_{n}=b$. In each interval $\left[t_{i-1}, t_{i}\right)_{\mathbb{T}}$ with $1 \leq i \leq n$, we choose an arbitrary point $\xi_{i}$ and form the sum

$$
S=\sum_{i=1}^{n} f\left(\xi_{i}\right)\left(t_{i}-t_{i-1}\right)
$$

$S$ is called a Riemann $\Delta$-sum of $f$ corresponding to $P \in \mathcal{P}$.

We say that $f$ is Riemann $\Delta$-integrable on $[a, b]_{\mathbb{T}}$, if there exists a number $I$ with the 
following property: for every $\varepsilon>0$, there exists $\delta>0$ such that

$$
|S-I|<\varepsilon,
$$

for every Riemann $\Delta$-sum $S$ of $f$ corresponding to any $P \in \mathcal{P}_{\delta}(a, b)$ and independent of $\xi_{i} \in\left[t_{i-1}, t_{i}\right)_{\mathbb{T}}$ for $1 \leq i \leq n$. It is clear that such a number $I$ is unique and is the Riemann $\Delta$-integral of $f$ from $a$ to $b$.

We point out that this definition makes sense only if for every $\delta>0$, there exists at least one partition $P \in \mathcal{P}_{\delta}(a, b)$. This is the content of the next result. For a proof of it, the reader may consult [18], Lemma 5.7.

Lemma 3.16. For every $\delta>0$, there exists some partition $P \in \mathcal{P}(a, b)$ given by $a=t_{0}<$ $t_{1}<\ldots<t_{n}=b$ such that for each $i \in\{1,2, \ldots, n\}$ either

$$
t_{i}-t_{i-1} \leq \delta
$$

or

$$
t_{i}-t_{i-1}>\delta \quad \text { and } \quad \rho\left(t_{i}\right)=t_{i-1} .
$$

In the definition of the Riemann $\Delta$-integral, we can suppress the restriction $a<b$ assuming the following equalities:

$$
\int_{a}^{a} f(t) \Delta t=0 \quad \text { and } \quad \int_{a}^{b} f(t) \Delta t=-\int_{b}^{a} f(t) \Delta t, \quad \text { if } \quad a>b .
$$

If $f$ is a bounded function, the previous definition concerning Riemann $\Delta$-integral is equivalent to definition of Darboux $\Delta$-integral. This is the content of the next result.

Theorem 3.17 ([18], Theorem 5.11). A bounded function $f$ on $[a, b]_{\mathbb{T}}$ is Riemann $\Delta$ integrable, if and only if, it is Darboux $\Delta$-integrable, in which case the values of the integrals are equal.

The next result concerns the $\Delta$-integrability of constant functions. It can be found in [18], Theorem 5.12.

Theorem 3.18. Assume that $a, b \in \mathbb{T}$. Every constant function $f: \mathbb{T} \rightarrow \mathbb{R}$ is $\Delta$-integrable from $a$ to $b$ and

$$
\int_{a}^{b} c \Delta t=c(b-a)
$$


Now, we present a theorem which summarizes the properties of $\Delta$-integrals. See [17], Theorem 1.77.

Theorem 3.19. If $a, b, c \in \mathbb{T}, \alpha \in \mathbb{R}$ and $f, g \in \mathcal{C}_{r d}$, then

(i) $\int_{a}^{b}[f(t)+g(t)] \Delta t=\int_{a}^{b} f(t) \Delta t+\int_{a}^{b} g(t) \Delta t$;

(ii) $\int_{a}^{b}(\alpha f)(t) \Delta t=\alpha \int_{a}^{b} f(t) \Delta t$

(iii) $\int_{a}^{b} f(t) \Delta t=-\int_{b}^{a} f(t) \Delta t$;

(iv) $\int_{a}^{b} f(t) \Delta t=\int_{a}^{c} f(t) \Delta t+\int_{c}^{b} f(t) \Delta t$;

(v) $\int_{a}^{b} f(\sigma(t)) g^{\Delta}(t) \Delta t=(f g)(b)-(f g)(a)-\int_{a}^{b} f^{\Delta}(t) g(t) \Delta t$;

(vi) $\int_{a}^{b} f(t) g^{\Delta}(t) \Delta t=(f g)(b)-(f g)(a)-\int_{a}^{b} f^{\Delta}(t) g(\sigma(t)) \Delta t$;

(vii) $\int_{a}^{a} f(t) \Delta t=0$

(viii) If $|f(t)| \leq g(t)$ on $[a, b)_{\mathbb{T}}$, then

$$
\left|\int_{a}^{b} f(t) \Delta t\right| \leq \int_{a}^{b} g(t) \Delta t
$$

(ix) If $f(t) \geq 0$ for every $a \leq t<b$, then $\int_{a}^{b} f(s) \Delta t \geq 0$.

Remark 3.20. The previous theorem remains true when we replace the hypothesis of rd-continuity of functions $f$ and $g$ by their $\Delta$-integrability.

The next theorem is a useful result. A proof of it can be found in [18], Theorem 5.13.

Theorem 3.21. Let $f: \mathbb{T} \rightarrow \mathbb{R}$ and $t \in \mathbb{T}$. Then $f$ is integrable from $t$ to $\sigma(t)$ and

$$
\int_{t}^{\sigma(t)} f(s) \Delta s=\mu(t) f(t) .
$$

In the sequel, we present an example borrowed from [18]. 
Example 3.22. Let $a, b \in \mathbb{T}$. If $\mathbb{T}=h \mathbb{Z}$, then every function $f$ defined on $h \mathbb{Z}$ is $\Delta$ integrable from $a$ to $b$ and

$$
\int_{a}^{b} f(t) \Delta t=\left\{\begin{array}{l}
\sum_{t=a / h}^{b / h-1} f(k h) h, \quad \text { if } \quad a<b, \\
0, \quad \text { if } a=b, \\
-\sum_{t=b / h}^{a / h-1} f(k h) h, \quad \text { if } a>b .
\end{array}\right.
$$

Let $a<b$. Then $b=a+p h$, for some $p \in \mathbb{N}$. Consider $P^{*} \in \mathcal{P}(a, b)$ given by

$$
a=t_{0}<t_{1}<\ldots<t_{p}=b
$$

where $t_{k}=a+k h$, for $0 \leq k \leq p$. Then, $P^{*}$ contains all points of $[a, b]_{\mathbb{T}}$ and $\left[t_{i-1}, t_{i}\right)_{\mathbb{T}}=$ $\left\{t_{i-1}\right\}$ for all $1 \leq i \leq p$. Also,

$$
U\left(f, P^{*}\right)=\sum_{i=1}^{p} M_{i}\left(t_{i}-t_{i-1}\right)=\sum_{i=1}^{p} f\left(t_{i-1}\right) h
$$

and

$$
L\left(f, P^{*}\right)=\sum_{i=1}^{p} m_{i}\left(t_{i}-t_{i-1}\right)=\sum_{i=1}^{p} f\left(t_{i-1}\right) h,
$$

which implies

$$
\begin{aligned}
U\left(f, P^{*}\right)=L\left(f, P^{*}\right) & =\sum_{i=1}^{p} f\left(t_{i-1}\right) h=\sum_{i=1}^{p} f(a+(i-1) h) h \\
& =\sum_{i=1}^{p} f\left(\frac{a}{h} h+(i-1) h\right) h=\sum_{i=1}^{p} f\left(\left(\frac{a}{h}+(i-1)\right) h\right) h \\
& =\sum_{k=a / h}^{b / h-1} f(k h) h .
\end{aligned}
$$

Hence, $f$ is integrable from $a$ to $b$ and

$$
\int_{a}^{b} f(t) \Delta t=\sum_{k=a / h}^{b / h-1} f(k h) h
$$


for the case $b>a$. Now, suppose $b=a$. Then (3.3.2) implies

$$
\int_{a}^{b} f(t) \Delta t=0
$$

Finally, if $a>b$, then

$$
\int_{a}^{b} f(t) \Delta t=-\int_{b}^{a} f(t) \Delta t=-\left[\sum_{k=b / h}^{a / h-1} f(k h) h\right]
$$

and the result follows.

In what follows, we present some examples which illustrate the indefinite $\Delta$-integrals. They can be found in [17].

Example 3.23. If $\mathbb{T}=\mathbb{Z}$, we evaluate the indefinite $\Delta$-integral

$$
\int a^{t} \Delta t
$$

where $a \neq 1$ is a constant. Since

$$
\left(\frac{a^{t}}{a-1}\right)^{\Delta}=\Delta\left(\frac{a^{t}}{a-1}\right)=\frac{a^{t+1}-a^{t}}{a-1}=a^{t},
$$

then

$$
\int a^{t} \Delta t=\frac{a^{t}}{a-1}+C
$$

where $C$ is an arbitrary constant.

Example 3.24. If $\mathbb{T}=\mathbb{Z}$, we evaluate the indefinite $\Delta$-integral

$$
\int(t+\alpha) \Delta t
$$

where $\alpha \in \mathbb{R}$. We have

$$
\begin{gathered}
\left(\frac{(t+\alpha)^{2}}{2}-\frac{t}{2}\right)^{\Delta}=\Delta\left(\frac{(t+\alpha)^{2}}{2}-\frac{t}{2}\right)=\left(\frac{(t+1+\alpha)^{2}-(t+1)-(t+\alpha)^{2}+t}{2}\right) \\
=\frac{(t+1+\alpha+t+\alpha)(t+1+\alpha-t-\alpha)-1}{2}=\frac{2 t+2 \alpha+1-1}{2}=t+\alpha .
\end{gathered}
$$


Then,

$$
\int(t+\alpha) \Delta t=\frac{(t+\alpha)^{2}}{2}-\frac{t}{2}+C
$$

where $C$ is an arbitrary constant.

The next result summarizes the $\Delta$-integrability of certain functions. For a proof of this result, see [18], Theorem 5.18, Theorem 5.19, Theorem 5.20 and Theorem 5.21.

Theorem 3.25. Let $a, b \in \mathbb{T}$. The following assertions hold:

1. Every continuous function $f$ on $[a, b]_{\mathbb{T}}$ is $\Delta$-integrable.

2. Every bounded function on $[a, b]_{\mathbb{T}}$ with only finitely many discontinuities is $\Delta$-integrable.

3. Every regulated function $f$ on $[a, b]_{\mathbb{T}}$ is $\Delta$-integrable.

4. Every monotone function on $[a, b]_{\mathbb{T}}$ is $\Delta$-integrable.

Finally, we present the Fundamental Theorem of Calculus for Riemann $\Delta$-integrals. It can be found in [18], Theorems 5.34 and 5.36 .

Theorem 3.26 (Fundamental Theorem of Calculus - Part I). Let $g$ be a continuous function on $[a, b]_{\mathbb{T}}$ such that $g$ is $\Delta$-differentiable on $[a, b)_{\mathbb{T}}$. If $g^{\Delta}$ is $\Delta$-integrable from a to $b$, then

$$
\int_{a}^{b} g^{\Delta}(t) \Delta t=g(b)-g(a) .
$$

Theorem 3.27 (Fundamental Theorem of Calculus, Part II). Let $f$ be a function which is $\Delta$-integrable from a to $b$. For $t \in[a, b]_{\mathbb{T}}$, define

$$
F(t)=\int_{a}^{t} f(s) \Delta s
$$

Then $F$ is continuous on $[a, b]_{\mathbb{T}}$. If $t_{0} \in[a, b)_{\mathbb{T}}$ and if $f$ is continuous at $t_{0}$ provided $t_{0}$ is right-dense, then $F$ is $\Delta$-differentiable at $t_{0}$ and

$$
F^{\Delta}\left(t_{0}\right)=f\left(t_{0}\right)
$$




\subsection{Kurzweil-Henstock delta integrals}

In this section, we present the Kurzweil-Henstock delta-integral and its most important properties. This concept is relatively new and the literature concerning it is scarce. In 2005, this concept was introduced for the first time by A. Peterson and B. Thompson in [90]. This section is based on this reference.

Let $\mathbb{T}$ be a time scale and consider the time scale interval $[a, b]_{\mathbb{T}}$ with $a, b \in \mathbb{T}$.

We say that $\delta=\left(\delta_{L}, \delta_{R}\right)$ is a $\Delta$-gauge of $[a, b]_{\mathbb{T}}$, provided $\delta_{L}(t)>0$ on $(a, b]_{\mathbb{T}}, \delta_{R}(t)>0$ on $[a, b)_{\mathbb{T}}, \delta_{L}(a) \geq 0, \delta_{R}(b) \geq 0$ and $\delta_{R}(t) \geq \mu(t)$ for all $t \in[a, b)_{\mathbb{T}}$.

We say that $P$ is a tagged partition of $[a, b]_{\mathbb{T}}$ if

$$
P=\left\{a=t_{0} \leq \xi_{1} \leq t_{1} \leq \ldots \leq t_{n-1} \leq \xi_{n} \leq t_{n}=b\right\}
$$

with $t_{i}>t_{i-1}$ for $1 \leq i \leq n$ and $t_{i}, \xi_{i} \in \mathbb{T}$. We call the points $\xi_{i}$ "tag points" and the points $t_{i}$ "end points". We denote such a tagged partition by $P=\left\{\left[t_{i-1}, t_{i}\right]_{\mathbb{T}}: \xi_{i}\right\}$, where $\left[t_{i-1}, t_{i}\right]_{\mathbb{T}}$ denotes a usual interval in $P$ and $\xi_{i}$ is the associated tag point in $\left[t_{i-1}, t_{i}\right]_{\mathbb{T}}$.

If $\delta$ is a $\Delta$-gauge for $[a, b]_{\mathbb{T}}$, then a partition $P$ is called $\delta$-fine if

$$
\xi_{i}-\delta_{L}\left(\xi_{i}\right) \leq t_{i-1}<t_{i} \leq \xi_{i}+\delta_{R}\left(\xi_{i}\right)
$$

for $1 \leq i \leq n=n(P)$.

In what follows, we define the Kurzweil-Henstock delta integral.

Definition 3.28. We say that $f:[a, b]_{\mathbb{T}} \rightarrow \mathbb{R}$ is Kurzweil-Henstock delta integrable (or $\mathrm{KH}$ delta-integrable) on $[a, b]_{\mathbb{T}}$, if there is a number $I$ such that given $\varepsilon>0$ there exists a $\Delta$-gauge $\delta$ of $[a, b]_{\mathbb{T}}$ such that

$$
\left|I-\sum_{i=1}^{n} f\left(\xi_{i}\right)\left(t_{i}-t_{i-1}\right)\right|<\varepsilon
$$

for all $\delta$-fine tagged partitions $P$ of $[a, b]_{\mathbb{T}}$. In this case, we write $I=K H \int_{a}^{b} f(t) \Delta t$.

Clearly, Definition 3.28 only makes sense if, for a given $\Delta$-gauge $\delta$ on $[a, b]_{\mathbb{T}}$, there exists at least one $\delta$-fine tagged partition $P$ of $[a, b]_{\mathbb{T}}$. This is the content of next result. It is a generalization of Cousin Lemma for a $\Delta$-gauge of a time scale interval. 
Lemma 3.29 ([90], Lemma 1.9). If $\delta$ is a $\Delta$-gauge on $[a, b]_{\mathbb{T}}$, then there is a $\delta$-fine tagged partition $P$ of $[a, b]_{\mathbb{T}}$.

Finally, we state a result which establishes fundamentals properties on KurzweilHenstock $\Delta$-integrals. It can be found in [90].

Theorem 3.30. Let $f:[a, b]_{\mathbb{T}} \rightarrow \mathbb{R}$ be a function. Then $f$ is KH-delta integrable on $[a, b]_{\mathbb{T}}$, if and only if, $f$ is KH-delta integrable on $[a, c]_{\mathbb{T}}$ and $[c, b]_{\mathbb{T}}$. In this case,

$$
K H \int_{a}^{b} f(t) \Delta t=K H \int_{a}^{c} f(t) \Delta t+K H \int_{c}^{b} f(t) \Delta t .
$$

Moreover, if $f, g:[a, b]_{\mathbb{T}} \rightarrow \mathbb{R}$ are KH-delta integrable on $[a, b]_{\mathbb{T}}$, then $\alpha f+\beta g$ is KH-delta integrable on $[a, b]_{\mathbb{T}}$ and

$$
K H \int_{a}^{b}(\alpha f(t)+\beta g(t)) \Delta t=\alpha\left(K H \int_{a}^{b} f(t) \Delta t\right)+\beta\left(K H \int_{a}^{b} f(t) \Delta t\right) .
$$





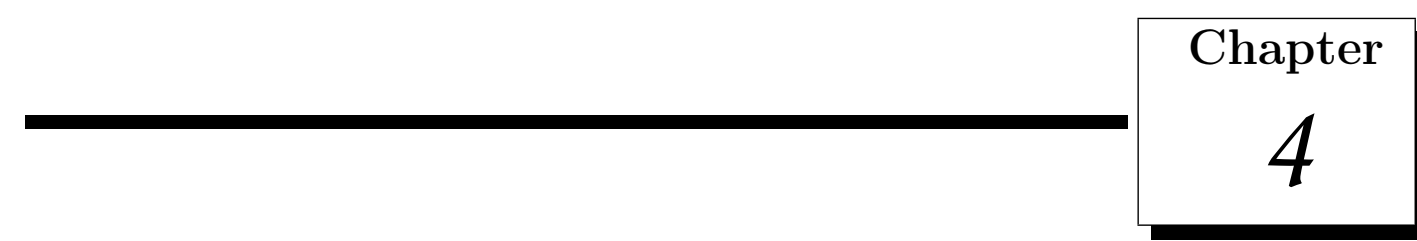

\section{Correspondences between equations}

In this chapter, we present correspondences between various types of equations. These correspondences will be fundamental to our purposes.

In the present work, our aim is to develop the theory of impulsive functional dynamic equations on time scales. However, as it was shown in Chapter 3, it is not easy to deal directly with the theory of dynamic equations on time scales, since it requires a new knowledge of calculus, which is not simple to work with. Thus, to facilitate dealing with impulsive functional dynamic equations on time scales and to obtain good results for them, we prove some correspondences between their solutions and solutions of an impulsive measure functional differential equation. Also, we establish a correspondence between these equations and measure functional differential equations and, finally, we prove that impulsive functional dynamic equations on time scales represent a special case of impulsive measure functional differential equations.

Then, we reduce our problem in such way that instead of working directly with impulsive functional dynamic equations on time scales, we can prove the results for measure functional differential equations and, then, translate them to impulsive functional dynamic equations on time scales. On the other hand, dealing directly with measure functional differential equations is not simple, since they involve Stieltjes-type integrals or distributional derivatives.

Because generalized ordinary differential equations are easier to deal with, we relate the solutions of a measure functional differential equation and the solutions of a generalized ordinary differential equation. Thus, we obtain an indirect correspondence between 
generalized ODEs and impulsive functional dynamic equations on time scales, which allows us to translate the known results from the theory of generalized ODEs to measure FDEs and to impulsive functional dynamic equations on time scales.

The majority of the results presented in this chapter are new and they can be found in the papers [36] and [37]. Also, to write this chapter, we use as main references the papers [98] and [100].

This chapter is divided in five sections. The first section presents the measure functional differential equations. In the second one, we present the correspondence between generalized ODEs and measure FDEs. The third one is devoted to proving that functional dynamic equations on time scales is a special case of measure FDEs. In fourth section, we establish a correspondence between measure FDEs and impulsive measure FDEs, showing that these last ones are a particular case of measure FDEs. Finally, in the fifth section, we establish a correspondence between impulsive functional dynamic equations on time scales and impulsive measure FDEs.

\subsection{Measure FDEs}

The theory of retarded functional differential equations is a branch of the theory of functional differential equations concerned with problems of the form

$$
\dot{x}=f\left(x_{t}, t\right), \quad t \in\left[t_{0}, t_{0}+\sigma\right]
$$

where $r$ and $\sigma>0$ are given numbers, $t_{0} \in \mathbb{R}$ and $f: P \times\left[t_{0}, t_{0}+\sigma\right] \rightarrow \mathbb{R}^{n}$, where $P \subset G\left([-r, 0], \mathbb{R}^{n}\right)$, and $x_{t}$ is given by $x_{t}(\theta)=x(t+\theta), \theta \in[-r, 0]$, for every $t \in\left[t_{0}, t_{0}+\sigma\right]$. Its equivalent integral form is given by

$$
x(t)=x\left(t_{0}\right)+\int_{t_{0}}^{t} f\left(x_{s}, s\right) d s, \quad t \in\left[t_{0}, t_{0}+\sigma\right],
$$

where the integral can be considered, for instance, in the sense of Riemann, Lebesgue or Kurzweil-Henstock. For more details, the reader may want to consult [57], [71] and [89].

In the present work, we focus our attention on more general equations of the form

$$
D x=f\left(x_{t}, t\right) D g, \quad t \in\left[t_{0}, t_{0}+\sigma\right]
$$

where $D x$ and $D g$ are the distributional derivatives, in the sense of L. Schwartz, of $x$ and 
$g$, respectively. We call these equations by measure functional differential equations.

While the theories of measure differential equations (including the more general abstract measure differential equations) and measure delay differential equations are welldeveloped (see [21], [24], [25], [26], [27], [28], [29], [30], [68], [69], [85], [96], [97], for instance), the literature concerning measure functional differential equations is scarce. See [19], [36] and [37]. These equations encompass various types of equations as we will prove later. This fact turns them a powerful tool to study other types of equations.

Also, it is a known fact that the measure functional differential equation given by

$$
\left\{\begin{array}{l}
D x=f\left(x_{t}, t\right) D g, \quad t \in\left[t_{0}, t_{0}+\sigma\right] \\
x_{t_{0}}=\phi
\end{array}\right.
$$

can be rewritten as follows

$$
\left\{\begin{array}{l}
x(t)=\phi(0)+\int_{t_{0}}^{t} f\left(x_{s}, s\right) \mathrm{d} g(s), \quad t \in\left[t_{0}, t_{0}+\sigma\right] \\
x_{t_{0}}=\phi
\end{array}\right.
$$

In the other words, the systems (4.1.1) and (4.1.2) are equivalent. Because of it, we will refer to (4.1.2) by measure functional differential equation. For more details, the reader may want to consult [24].

\subsection{Measure FDEs and generalized ODEs}

In this section, we present a correspondence between the solutions of a measure FDE and the solutions of a generalized ODE. This correspondence is essential to our purposes, since by applying it, we are able to translate the results from the theory of generalized ODEs to measure FDEs. It worth mentioning that all the results presented in this section are new and they can be found in [36].

Let $O \subset G\left(\left[t_{0}-r, t_{0}+\sigma\right], \mathbb{R}^{n}\right)$ and $P=\left\{y_{t} ; y \in O, t \in\left[t_{0}, t_{0}+\sigma\right]\right\} \subset G\left([-r, 0], \mathbb{R}^{n}\right)$. Consider a nondecreasing function $g:\left[t_{0}, t_{0}+\sigma\right] \rightarrow \mathbb{R}$ and a function $f: P \times\left[t_{0}, t_{0}+\sigma\right] \rightarrow$ $\mathbb{R}^{n}$. Here, we will show that, under certain assumptions, a measure functional differential equation of the form

$$
D y=f\left(y_{t}, t\right) D g
$$

where any solution $y:\left[t_{0}-r, t_{0}+\sigma\right] \rightarrow \mathbb{R}^{n}$ is supposed to be a regulated function, can be 
converted into a generalized ordinary differential equation of the form

$$
\frac{\mathrm{d} x}{\mathrm{~d} \tau}=D F(x, t)
$$

where $x$ takes values in $O$, that is, we transform the original measure functional differential equation (4.2.1), whose solutions take values in $\mathbb{R}^{n}$, into a generalized ordinary differential equation, whose solution takes values in an infinite-dimensional Banach space. The righthand side $F$ of such generalized equation will be given by

$$
F(x, t)(\vartheta)= \begin{cases}0, & t_{0}-r \leq \vartheta \leq t_{0}, \\ \int_{t_{0}}^{\vartheta} f\left(x_{s}, s\right) \mathrm{d} g(s), & t_{0} \leq \vartheta \leq t \leq t_{0}+\sigma, \\ \int_{t_{0}}^{t} f\left(x_{s}, s\right) \mathrm{d} g(s), & t \leq \vartheta \leq t_{0}+\sigma,\end{cases}
$$

for every $x \in O$ and $t \in\left[t_{0}, t_{0}+\sigma\right]$.

As we will show, the relation between the solution $x$ of (4.2.2) and the solution $y$ of (4.2.1) is described by

$$
x(t)(\vartheta)= \begin{cases}y(\vartheta), & \vartheta \in\left[t_{0}-r, t\right] \\ y(t), & \vartheta \in\left[t, t_{0}+\sigma\right]\end{cases}
$$

where $t \in\left[t_{0}, t_{0}+\sigma\right]$.

We introduce three conditions that the function $f: P \times\left[t_{0}, t_{0}+\sigma\right] \rightarrow \mathbb{R}^{n}$ must fulfill:

(A) The integral $\int_{t_{0}}^{t_{0}+\sigma} f\left(y_{t}, t\right) \mathrm{d} g(t)$ exists for every $y \in O$.

(B) There exists a constant $M>0$ such that

$$
\left|\int_{u}^{v} f\left(y_{s}, s\right) \mathrm{d} g(s)\right| \leq M[g(v)-g(u)]
$$

for every $y \in O$ and every $u, v \in\left[t_{0}, t_{0}+\sigma\right]$.

(C) There exists a constant $L>0$ such that

$$
\left|\int_{u}^{v}\left[f\left(y_{s}, s\right)-f\left(z_{s}, s\right)\right] \mathrm{d} g(s)\right| \leq L \int_{u}^{v}\left\|y_{s}-z_{s}\right\|_{\infty} \mathrm{d} g(s)
$$

for every $y, z \in O$ and every $u, v \in\left[t_{0}, t_{0}+\sigma\right]$. 
Before proceeding further, we need the following property of regulated functions. It is a new result and it can be found in [36].

Lemma 4.1. If $y:\left[t_{0}-r, t_{0}+\sigma\right] \rightarrow \mathbb{R}^{n}$ is a regulated function, then $s \mapsto\left\|y_{s}\right\|_{\infty}$ is regulated on $\left[t_{0}, t_{0}+\sigma\right]$.

Proof. We will show that $\lim _{s \rightarrow s_{0}-}\left\|y_{s}\right\|_{\infty}$ exists for every $s_{0} \in\left(t_{0}, t_{0}+\sigma\right]$. The function $y$ is regulated, and, therefore, satisfies the Cauchy condition at $s_{0}-r$ and $s_{0}$ : Given an arbitrary $\varepsilon>0$, there exists a $\delta \in\left(0, s_{0}-t_{0}\right)$ such that

$$
|y(u)-y(v)|<\varepsilon, \quad u, v \in\left(s_{0}-r-\delta, s_{0}-r\right)
$$

and

$$
|y(u)-y(v)|<\varepsilon, \quad u, v \in\left(s_{0}-\delta, s_{0}\right) \text {. }
$$

Now, consider a pair of numbers $s_{1}, s_{2}$ such that $s_{0}-\delta<s_{1}<s_{2}<s_{0}$. For every $s \in\left[s_{1}-r, s_{2}-r\right]$, it follows from (4.2.5) that

$$
|y(s)|<\left|y\left(s_{2}-r\right)\right|+\varepsilon \leq\left\|y_{s_{2}}\right\|_{\infty}+\varepsilon .
$$

It is also clear that $|y(s)| \leq\left\|y_{s_{2}}\right\|_{\infty}$ for every $s \in\left[s_{2}-r, s_{1}\right]$. Consequently, $\left\|y_{s_{1}}\right\|_{\infty} \leq$ $\left\|y_{s_{2}}\right\|_{\infty}+\varepsilon$. Using (4.2.6) in a similar way, we obtain $\left\|y_{s_{2}}\right\|_{\infty} \leq\left\|y_{s_{1}}\right\|_{\infty}+\varepsilon$. It follows that

$$
\left|\left\|y_{s_{1}}\right\|_{\infty}-\left\|y_{s_{2}}\right\|_{\infty}\right| \leq \varepsilon, \quad s_{1}, s_{2} \in\left(s_{0}-\delta, s_{0}\right)
$$

that is, the Cauchy condition for the existence of $\lim _{s \rightarrow s_{0}-}\left\|y_{s}\right\|_{\infty}$ is satisfied.

The existence of $\lim _{s \rightarrow s_{0}+}\left\|y_{s}\right\|_{\infty}$ for $s_{0} \in\left[t_{0}, t_{0}+\sigma\right)$ can be proved similarly.

The next result ensures that if the function $f: P \times\left[t_{0}, t_{0}+\sigma\right] \rightarrow \mathbb{R}^{n}$ satisfies conditions $(\mathrm{A}),(\mathrm{B})$ and $(\mathrm{C})$ and $g:\left[t_{0}, t_{0}+\sigma\right] \rightarrow \mathbb{R}$ is nondecreasing, then $F$ belongs to the class $\mathcal{F}\left(O \times\left[t_{0}, t_{0}+\sigma\right], h\right)$, where $h(t)=(L+M)\left(g(t)-g\left(t_{0}\right)\right), t \in\left[t_{0}, t_{0}+\sigma\right]$.

Lemma 4.2. Let $O \subset G\left(\left[t_{0}-r, t_{0}+\sigma\right], \mathbb{R}^{n}\right)$ and $P=\left\{y_{t} ; y \in O, t \in\left[t_{0}, t_{0}+\sigma\right]\right\}$. Assume that $g:\left[t_{0}, t_{0}+\sigma\right] \rightarrow \mathbb{R}$ is a nondecreasing function and that $f: P \times\left[t_{0}, t_{0}+\sigma\right] \rightarrow \mathbb{R}^{n}$ satisfies conditions $(\mathrm{A}),(\mathrm{B})$ and $(\mathrm{C})$. Then the function $F: O \times\left[t_{0}, t_{0}+\sigma\right] \rightarrow G\left(\left[t_{0}-\right.\right.$ $\left.\left.r, t_{0}+\sigma\right], \mathbb{R}^{n}\right)$ given by (4.2.3) belongs to the class $\mathcal{F}\left(O \times\left[t_{0}, t_{0}+\sigma\right], h\right)$, where

$$
h(t)=(L+M)\left(g(t)-g\left(t_{0}\right)\right), \quad t \in\left[t_{0}, t_{0}+\sigma\right] .
$$


Proof. Condition (A) implies that the integrals in the definition of $F$ exist. Given $y \in O$ and $t_{0} \leq s_{1}<s_{2} \leq t_{0}+\sigma$, we have

$$
F\left(y, s_{2}\right)(\vartheta)-F\left(y, s_{1}\right)(\vartheta)=\left\{\begin{array}{l}
0, t_{0}-r \leq \vartheta \leq s_{1}, \\
\int_{s_{1}}^{\vartheta} f\left(y_{s}, s\right) \mathrm{d} g(s), \quad s_{1} \leq \vartheta \leq s_{2}, \\
\int_{s_{1}}^{s_{2}} f\left(y_{s}, s\right) \mathrm{d} g(s), \quad s_{2} \leq \vartheta \leq t_{0}+\sigma .
\end{array}\right.
$$

Hence, for an arbitrary $y \in O$ and for $t_{0} \leq s_{1}<s_{2} \leq t_{0}+\sigma$, it follows by condition (B)

$$
\begin{gathered}
\left\|F\left(y, s_{2}\right)-F\left(y, s_{1}\right)\right\|_{\infty}=\sup _{t_{0}-r \leq \vartheta \leq t_{0}+\sigma}\left|F\left(y, s_{2}\right)(\vartheta)-F\left(y, s_{1}\right)(\vartheta)\right|= \\
=\sup _{s_{1} \leq \vartheta \leq s_{2}}\left|F\left(y, s_{2}\right)(\vartheta)-F\left(y, s_{1}\right)(\vartheta)\right|=\sup _{s_{1} \leq \vartheta \leq s_{2}}\left|\int_{s_{1}}^{\vartheta} f\left(y_{s}, s\right) \mathrm{d} g(s)\right| \leq \\
\leq M\left[g\left(s_{2}\right)-g\left(s_{1}\right)\right] \leq h\left(s_{2}\right)-h\left(s_{1}\right) .
\end{gathered}
$$

Similarly, using (C), if $y, z \in O$ and $t_{0} \leq s_{1} \leq s_{2} \leq t_{0}+\sigma$, then

$$
\begin{gathered}
\left\|F\left(y, s_{2}\right)-F\left(y, s_{1}\right)-F\left(z, s_{2}\right)+F\left(z, s_{1}\right)\right\|_{\infty}= \\
=\sup _{s_{1} \leq \vartheta \leq s_{2}}\left|\int_{s_{1}}^{\vartheta}\left[f\left(y_{s}, s\right)-f\left(z_{s}, s\right)\right] \mathrm{d} g(s)\right| \leq L \sup _{s_{1} \leq \vartheta \leq s_{2}} \int_{s_{1}}^{\vartheta}\left\|y_{s}-z_{s}\right\|_{\infty} \mathrm{d} g(s) \leq \\
\leq\|y-z\|_{\infty} \int_{s_{1}}^{s_{2}} L \mathrm{~d} g(s) \leq\|y-z\|_{\infty}\left[h\left(s_{2}\right)-h\left(s_{1}\right)\right]
\end{gathered}
$$

(note that the function $s \mapsto\left\|y_{s}-z_{s}\right\|_{\infty}$ is regulated according to Lemma 4.1, and therefore the integral $\int_{s_{1}}^{\vartheta} L\left\|y_{s}-z_{s}\right\|_{\infty} \mathrm{d} g(s)$ exists).

In what follows, we present a property which will be important for us, because it ensures that if $y \in O$, then $x(t) \in O$ for every $t \in\left[t_{0}, t_{0}+\sigma\right]$ and for $y$ and $x$ related by $(4.2 .4)$.

Definition 4.3. Let $O$ be a subset of $G\left(\left[t_{0}-r, t_{0}+\sigma\right], \mathbb{R}^{n}\right)$. We will say that $O$ has the prolongation property, if for every $y \in O$ and every $\bar{t} \in\left[t_{0}-r, t_{0}+\sigma\right]$, the function $\bar{y}$ given by

$$
\bar{y}(t)= \begin{cases}y(t), & t_{0}-r \leq t \leq \bar{t} \\ y(\bar{t}), & \bar{t}<t \leq t_{0}+\sigma\end{cases}
$$

is also an element of $O$. 
For example, let $B$ be an arbitrary subset of $\mathbb{R}^{n}$. Then both the set of all regulated functions $f:\left[t_{0}-r, t_{0}+\sigma\right] \rightarrow B$ and the set of all continuous functions $f:\left[t_{0}-r, t_{0}+\sigma\right] \rightarrow B$ have the prolongation property.

The following statement is a slightly modified version of Lemma 3.3 from [40] (which is concerned with the special case $g(t)=t$ ). However, the proof from [40] can be carried over without any changes and we repeat it here.

Lemma 4.4. Let $O$ be a subset of $G\left(\left[t_{0}-r, t_{0}+\sigma\right], \mathbb{R}^{n}\right)$ with the prolongation property and $P=\left\{y_{t} ; y \in O, t \in\left[t_{0}, t_{0}+\sigma\right]\right\}$. Assume that $\phi \in P, g:\left[t_{0}, t_{0}+\sigma\right] \rightarrow \mathbb{R}$ is a nondecreasing function and $f: P \times\left[t_{0}, t_{0}+\sigma\right] \rightarrow \mathbb{R}^{n}$ is such that the integral $\int_{t_{0}}^{t_{0}+\sigma} f\left(y_{t}, t\right) \mathrm{d} g(t)$ exists for every $y \in O$. Consider $F$ given by (4.2.3) and assume that $x:\left[t_{0}, t_{0}+\sigma\right] \rightarrow O$ is a solution of

$$
\frac{\mathrm{d} x}{\mathrm{~d} \tau}=D F(x, t)
$$

with initial condition

$$
x\left(t_{0}\right)(\vartheta)= \begin{cases}\phi\left(\vartheta-t_{0}\right), & \text { for } \vartheta \in\left[t_{0}-r, t_{0}\right], \\ x\left(t_{0}\right)\left(t_{0}\right), & \text { for } \vartheta \in\left[t_{0}, t_{0}+\sigma\right] .\end{cases}
$$

If $v \in\left[t_{0}, t_{0}+\sigma\right]$ and $\vartheta \in\left[t_{0}-r, t_{0}+\sigma\right]$, then

$$
x(v)(\vartheta)=x(v)(v), \quad \vartheta \geq v
$$

and

$$
x(v)(\vartheta)=x(\vartheta)(\vartheta), \quad v \geq \vartheta
$$

Proof. Assume that $\vartheta \geq v$. Since $x$ is a solution of

$$
\frac{\mathrm{d} x}{\mathrm{~d} \tau}=D F(x, t)
$$

we have

$$
x(v)(v)=x\left(t_{0}\right)(v)+\int_{t_{0}}^{v} D F(x(\tau), t)(v)
$$

and, similarly,

$$
x(v)(\vartheta)=x\left(t_{0}\right)(\vartheta)+\int_{t_{0}}^{v} D F(x(\tau), t)(\vartheta) .
$$

Since $x\left(t_{0}\right)(\vartheta)=x\left(t_{0}\right)(v)$, by the properties of the initial condition, we obtain

$$
x(v)(\vartheta)-x(v)(v)=\int_{t_{0}}^{v} D F(x(\tau), t)(\vartheta)-\int_{t_{0}}^{v} D F(x(\tau), t)(v) .
$$


It follows from the existence of the integral $\int_{t_{0}}^{v} D F(x(\tau), t)$ that for every $\varepsilon>0$, there is a gauge $\delta$ on $\left[t_{0}, t_{0}+\sigma\right]$ such that if $\left\{\left(\tau_{i},\left[s_{i-1}, s_{i}\right]\right), i=1, \ldots, k\right\}$ is a $\delta$-fine tagged partition of $\left[t_{0}, v\right]$, then

$$
\left\|\sum_{i=1}^{k}\left(F\left(x\left(\tau_{i}\right), s_{i}\right)-F\left(x\left(\tau_{i}\right), s_{i-1}\right)\right)-\int_{t_{0}}^{v} D F(x(\tau), t)\right\|_{\infty}<\varepsilon .
$$

Therefore, we have

$$
\begin{gathered}
\|x(v)(\vartheta)-x(v)(v)\|<2 \varepsilon+ \\
+\left\|\sum_{i=1}^{k}\left(F\left(x\left(\tau_{i}\right), s_{i}\right)-F\left(x\left(\tau_{i}\right), s_{i-1}\right)\right)(\vartheta)-\sum_{i=1}^{k}\left(F\left(x\left(\tau_{i}\right), s_{i}\right)-F\left(x\left(\tau_{i}\right), s_{i-1}\right)\right)(v)\right\| .
\end{gathered}
$$

By the definition of $F$ in (4.2.3), it is a matter of routine to check that, for every $i \in\{1, \ldots, k\}$, we have

$$
F\left(x\left(\tau_{i}\right), s_{i}\right)(\vartheta)-F\left(x\left(\tau_{i}\right), s_{i-1}\right)(\vartheta)=F\left(x\left(\tau_{i}\right), s_{i}\right)(v)-F\left(x\left(\tau_{i}\right), s_{i-1}\right)(v),
$$

and, consequently,

$$
\|x(v)(\vartheta)-x(v)(v)\|<2 \varepsilon
$$

Since this holds for an arbitrary $\varepsilon>0$, the relation (4.2.7) is satisfied.

In order to prove the second statement, assume that $\vartheta \leq v$. Similarly to the first part of the proof, we have

$$
x(v)(\vartheta)=x\left(t_{0}\right)(\vartheta)+\int_{t_{0}}^{v} D F(x(\tau), t)(\vartheta)
$$

and

$$
x(\vartheta)(\vartheta)=x\left(t_{0}\right)(\vartheta)+\int_{t_{0}}^{\vartheta} D F(x(\tau), t)(\vartheta)
$$

Hence,

$$
x(v)(\vartheta)-x(\vartheta)(\vartheta)=\int_{\vartheta}^{v} D F(x(\tau), t)(\vartheta)
$$

Now, if $\left\{\left(\tau_{i},\left[s_{i-1}, s_{i}\right]\right), i=1, \ldots, k\right\}$ is an arbitrary tagged partition of $[\vartheta, v]$, it is straightforward to check by (4.2.3) that, for every $i \in\{1, \ldots, k\}$, we have

$$
F\left(x\left(\tau_{i}\right), s_{i}\right)(\vartheta)-F\left(x\left(\tau_{i}\right), s_{i-1}\right)(\vartheta)=0
$$

This means that $\int_{\vartheta}^{v} D F(x(\tau), t)(\vartheta)=0$ and $x(v)(\vartheta)=x(\vartheta)(\vartheta)$. 
The following two theorems describe the correspondence between the solutions of a generalized ordinary differential equation and solutions of a measure functional differential equation. They are the most important results in this section. Their proofs are inspired by similar proofs from the paper [40], which describes the special case $g(t)=t$, that is, the usual type of functional differential equations. These results are new and are contained in $[36]$.

Theorem 4.5. Assume that $X$ is a closed subspace of $G\left(\left[t_{0}-r, t_{0}+\sigma\right], \mathbb{R}^{n}\right), O$ is a subset of $X$ with the prolongation property, $P=\left\{x_{t} ; x \in O, t \in\left[t_{0}, t_{0}+\sigma\right]\right\}, \phi \in P$, $g:\left[t_{0}, t_{0}+\sigma\right] \rightarrow \mathbb{R}$ is a nondecreasing function and $f: P \times\left[t_{0}, t_{0}+\sigma\right] \rightarrow \mathbb{R}^{n}$ satisfies conditions (A), (B) and (C). Let $F: O \times\left[t_{0}, t_{0}+\sigma\right] \rightarrow G\left(\left[t_{0}-r, t_{0}+\sigma\right], \mathbb{R}^{n}\right)$ be given by (4.2.3) and assume that $F(x, t) \in X$ for every $x \in O, t \in\left[t_{0}, t_{0}+\sigma\right]$. Let $y \in O$ be a solution of the measure functional differential equation

$$
\left\{\begin{array}{l}
D y=f\left(y_{t}, t\right) D g, \quad t \in\left[t_{0}, t_{0}+\sigma\right] \\
y_{t_{0}}=\phi
\end{array}\right.
$$

For every $t \in\left[t_{0}-r, t_{0}+\sigma\right]$, let

$$
x(t)(\vartheta)= \begin{cases}y(\vartheta), & \vartheta \in\left[t_{0}-r, t\right], \\ y(t), & \vartheta \in\left[t, t_{0}+\sigma\right] .\end{cases}
$$

Then the function $x:\left[t_{0}, t_{0}+\sigma\right] \rightarrow O$ is a solution of the generalized ordinary differential equation

$$
\frac{\mathrm{d} x}{\mathrm{~d} \tau}=D F(x, t)
$$

with the initial condition

$$
x\left(t_{0}\right)(\vartheta)=\left\{\begin{array}{l}
\phi\left(\vartheta-t_{0}\right), \quad t_{0}-r \leq \vartheta \leq t_{0}, \\
x\left(t_{0}\right)\left(t_{0}\right), \quad t_{0} \leq \vartheta \leq t_{0}+\sigma .
\end{array}\right.
$$

Proof. We will show that, for every $v \in\left[t_{0}, t_{0}+\sigma\right]$, the integral $\int_{t_{0}}^{v} D F(x(\tau), t)$ exists and

$$
x(v)-x\left(t_{0}\right)=\int_{t_{0}}^{v} D F(x(\tau), t) .
$$

Let an arbitrary $\varepsilon>0$ be given. Since $g$ is nondecreasing, it can have only a finite number of points $t \in\left[t_{0}, v\right]$ such that $\Delta^{+} g(t) \geq \varepsilon / M$. Denote these points by $t_{1}, \ldots, t_{m}$. 
Consider a gauge $\delta:\left[t_{0}, t_{0}+\sigma\right] \rightarrow \mathbb{R}^{+}$such that

$$
\delta(\tau)<\min \left\{\frac{t_{k}-t_{k-1}}{2}, k=2, \ldots, m\right\}, \tau \in\left[t_{0}, t_{0}+\sigma\right]
$$

and

$$
\delta(\tau)<\min \left\{\left|\tau-t_{k}\right|,\left|\tau-t_{k-1}\right|, \tau \in\left(t_{k-1}, t_{k}\right) ; k=2, \ldots, m\right\} .
$$

These conditions ensure that if a point-interval pair $(\tau,[c, d])$ is $\delta$-fine, then $[c, d]$ contains at most one of the points $t_{1}, \ldots, t_{m}$, and, moreover, $\tau=t_{k}$ whenever $t_{k} \in[c, d]$.

Since $y_{t_{k}}=x\left(t_{k}\right)_{t_{k}}$, it follows from Corollary 2.7 that

$$
\lim _{s \rightarrow t_{k}+} \int_{t_{k}}^{s} L\left\|y_{s}-x\left(t_{k}\right)_{s}\right\|_{\infty} \mathrm{d} g(s)=L\left\|y_{t_{k}}-x\left(t_{k}\right)_{t_{k}}\right\|_{\infty} \Delta^{+} g\left(t_{k}\right)=0
$$

for every $k \in\{1, \ldots, m\}$. Thus, the gauge $\delta$ might be chosen in such a way that

$$
\int_{t_{k}}^{t_{k}+\delta\left(t_{k}\right)} L\left\|y_{s}-x\left(t_{k}\right)_{s}\right\|_{\infty} \mathrm{d} g(s)<\frac{\varepsilon}{2 m+1}, \quad k \in\{1, \ldots, m\} .
$$

Using Corollary 2.7 again, we have

$$
\|y(\tau+)-y(\tau)\|=\left\|f\left(y_{\tau}, \tau\right) \Delta^{+} g(\tau)\right\|<M \frac{\varepsilon}{M}=\varepsilon, \quad \tau \in\left[t_{0}, t_{0}+\sigma\right] \backslash\left\{t_{1}, \ldots, t_{m}\right\} .
$$

Thus, we can assume that the gauge $\delta$ is such that

$$
\|y(\rho)-y(\tau)\| \leq \varepsilon
$$

for every $\tau \in\left[t_{0}, t_{0}+\sigma\right] \backslash\left\{t_{1}, \ldots, t_{m}\right\}$ and $\rho \in[\tau, \tau+\delta(\tau))$.

Assume now that $\left\{\left(\tau_{i},\left[s_{i-1}, s_{i}\right]\right), i=1, \ldots, l\right\}$ is a $\delta$-fine tagged partition of the interval $\left[t_{0}, v\right]$. Using the definition of $x$, it can be easily shown that

$$
\left[x\left(s_{i}\right)-x\left(s_{i-1}\right)\right](\vartheta)= \begin{cases}0, & \vartheta \in\left[t_{0}-r, s_{i-1}\right], \\ \int_{s_{i-1}}^{\vartheta} f\left(y_{s}, s\right) \mathrm{d} g(s), & \vartheta \in\left[s_{i-1}, s_{i}\right], \\ \int_{s_{i-1}}^{s_{i}} f\left(y_{s}, s\right) \mathrm{d} g(s), & \vartheta \in\left[s_{i}, t_{0}+\sigma\right] .\end{cases}
$$


Similarly, it follows from the definition of $F$ that

$$
\left[F\left(x\left(\tau_{i}\right), s_{i}\right)-F\left(x\left(\tau_{i}\right), s_{i-1}\right)\right](\vartheta)= \begin{cases}0, & \vartheta \in\left[t_{0}-r, s_{i-1}\right], \\ \int_{s_{i-1}}^{\vartheta} f\left(x\left(\tau_{i}\right)_{s}, s\right) \mathrm{d} g(s), & \vartheta \in\left[s_{i-1}, s_{i}\right], \\ \int_{s_{i-1}}^{s_{i}} f\left(x\left(\tau_{i}\right)_{s}, s\right) \mathrm{d} g(s), & \vartheta \in\left[s_{i}, t_{0}+\sigma\right] .\end{cases}
$$

By combination of the previous equalities, we obtain

$$
\begin{aligned}
& {\left[x\left(s_{i}\right)-x\left(s_{i-1}\right)\right](\vartheta)-\left[F\left(x\left(\tau_{i}\right), s_{i}\right)-F\left(x\left(\tau_{i}\right), s_{i-1}\right)\right](\vartheta)=} \\
& = \begin{cases}0, & \vartheta \in\left[t_{0}-r, s_{i-1}\right], \\
\int_{s_{i-1}}^{\vartheta}\left(f\left(y_{s}, s\right)-f\left(x\left(\tau_{i}\right)_{s}, s\right)\right) \mathrm{d} g(s), & \vartheta \in\left[s_{i-1}, s_{i}\right], \\
\int_{s_{i-1}}^{s_{i}}\left(f\left(y_{s}, s\right)-f\left(x\left(\tau_{i}\right)_{s}, s\right)\right) \mathrm{d} g(s), & \vartheta \in\left[s_{i}, t_{0}+\sigma\right] .\end{cases}
\end{aligned}
$$

Consequently,

$$
\begin{gathered}
\left\|x\left(s_{i}\right)-x\left(s_{i-1}\right)-\left[F\left(x\left(\tau_{i}\right), s_{i}\right)-F\left(x\left(\tau_{i}\right), s_{i-1}\right)\right]\right\|_{\infty}= \\
=\sup _{\vartheta \in\left[t_{0}-r, t_{0}+\sigma\right]}\left|\left[x\left(s_{i}\right)-x\left(s_{i-1}\right)\right](\vartheta)-\left[F\left(x\left(\tau_{i}\right), s_{i}\right)-F\left(x\left(\tau_{i}\right), s_{i-1}\right)\right](\vartheta)\right|= \\
=\sup _{\vartheta \in\left[s_{i-1}, s_{i}\right]}\left|\int_{s_{i-1}}^{\vartheta}\left[f\left(y_{s}, s\right)-f\left(x\left(\tau_{i}\right)_{s}, s\right)\right] \mathrm{d} g(s)\right| .
\end{gathered}
$$

By the definition of $x, x\left(\tau_{i}\right)_{s}=y_{s}$ whenever $s \leq \tau_{i}$. Thus,

$$
\int_{s_{i-1}}^{\vartheta}\left[f\left(y_{s}, s\right)-f\left(x\left(\tau_{i}\right)_{s}, s\right)\right] \mathrm{d} g(s)= \begin{cases}0, & \vartheta \in\left[s_{i-1}, \tau_{i}\right] \\ \int_{\tau_{i}}^{\vartheta}\left[f\left(y_{s}, s\right)-f\left(x\left(\tau_{i}\right)_{s}, s\right)\right] \mathrm{d} g(s), & \vartheta \in\left[\tau_{i}, s_{i}\right] .\end{cases}
$$

Then, condition (C) implies

$$
\left|\int_{\tau_{i}}^{\vartheta}\left[f\left(y_{s}, s\right)-f\left(x\left(\tau_{i}\right)_{s}, s\right)\right] \mathrm{d} g(s)\right| \leq L \int_{\tau_{i}}^{\vartheta}\left\|y_{s}-x\left(\tau_{i}\right)_{s}\right\|_{\infty} \mathrm{d} g(s) \leq L \int_{\tau_{i}}^{s_{i}}\left\|y_{s}-x\left(\tau_{i}\right)_{s}\right\|_{\infty} \mathrm{d} g(s) .
$$

Given a particular point-interval pair $\left(\tau_{i},\left[s_{i-1}, s_{i}\right]\right)$, there are two possibilities: 
(i) The intersection of $\left[s_{i-1}, s_{i}\right]$ and $\left\{t_{1}, \ldots, t_{m}\right\}$ contains a single point $t_{k}=\tau_{i}$.

(ii) The intersection of $\left[s_{i-1}, s_{i}\right]$ and $\left\{t_{1}, \ldots, t_{m}\right\}$ is empty.

In case (i), it follows from the definition of the gauge $\delta$ that

$$
L \int_{\tau_{i}}^{s_{i}}\left\|y_{s}-x\left(\tau_{i}\right)_{s}\right\|_{\infty} \mathrm{d} g(s) \leq \frac{\varepsilon}{2 m+1}
$$

that is,

$$
\left\|x\left(s_{i}\right)-x\left(s_{i-1}\right)-\left[F\left(x\left(\tau_{i}\right), s_{i}\right)-F\left(x\left(\tau_{i}\right), s_{i-1}\right)\right]\right\|_{\infty} \leq \frac{\varepsilon}{2 m+1} .
$$

In case (ii), we have

$$
\left\|y_{s}-x\left(\tau_{i}\right)_{s}\right\|_{\infty}=\sup _{\rho \in\left[\tau_{i}, s\right]}\left|y(\rho)-y\left(\tau_{i}\right)\right| \leq \varepsilon, \quad s \in\left[\tau_{i}, s_{i}\right]
$$

by the definition of the gauge $\delta$. Thus,

$$
\left\|x\left(s_{i}\right)-x\left(s_{i-1}\right)-\left[F\left(x\left(\tau_{i}\right), s_{i}\right)-F\left(x\left(\tau_{i}\right), s_{i-1}\right)\right]\right\|_{\infty} \leq \varepsilon L \int_{\tau_{i}}^{s_{i}} \mathrm{~d} g(s) .
$$

Combining cases (i) and (ii) and using the fact that case (i) occurs at most $2 m$ times, we obtain

$$
\left\|x(v)-x\left(t_{0}\right)-\sum_{i=1}^{l}\left[F\left(x\left(\tau_{i}\right), s_{i}\right)-F\left(x\left(\tau_{i}\right), s_{i-1}\right)\right]\right\|_{\infty} \leq \varepsilon L \int_{t_{0}}^{t_{0}+\sigma} \mathrm{d} g(s)+\frac{2 m \varepsilon}{2 m+1} .
$$

Since $\varepsilon>0$ is arbitrary, it follows that

$$
x(v)-x\left(t_{0}\right)=\int_{t_{0}}^{v} D F(x(\tau), t) .
$$

Theorem 4.6. Assume that $X$ is a closed subspace of $G\left(\left[t_{0}-r, t_{0}+\sigma\right], \mathbb{R}^{n}\right), O$ is a subset of $X$ with the prolongation property, $P=\left\{x_{t} ; x \in O, t \in\left[t_{0}, t_{0}+\sigma\right]\right\}, \phi \in P$, $g:\left[t_{0}, t_{0}+\sigma\right] \rightarrow \mathbb{R}$ is a nondecreasing function and $f: P \times\left[t_{0}, t_{0}+\sigma\right] \rightarrow \mathbb{R}^{n}$ satisfies conditions (A), (B) and (C). Let $F: O \times\left[t_{0}, t_{0}+\sigma\right] \rightarrow G\left(\left[t_{0}-r, t_{0}+\sigma\right], \mathbb{R}^{n}\right)$ be given by (4.2.3) and assume that $F(x, t) \in X$ for every $x \in O$ and $t \in\left[t_{0}, t_{0}+\sigma\right]$. Let $x$ : $\left[t_{0}, t_{0}+\sigma\right] \rightarrow O$ be a solution of the generalized ordinary differential equation

$$
\frac{\mathrm{d} x}{\mathrm{~d} \tau}=D F(x, t)
$$


with the initial condition

$$
x\left(t_{0}\right)(\vartheta)= \begin{cases}\phi\left(\vartheta-t_{0}\right), & t_{0}-r \leq \vartheta \leq t_{0} \\ x\left(t_{0}\right)\left(t_{0}\right), & t_{0} \leq \vartheta \leq t_{0}+\sigma .\end{cases}
$$

Then the function $y \in O$ defined by

$$
y(\vartheta)= \begin{cases}x\left(t_{0}\right)(\vartheta), & t_{0}-r \leq \vartheta \leq t_{0} \\ x(\vartheta)(\vartheta), & t_{0} \leq \vartheta \leq t_{0}+\sigma\end{cases}
$$

is a solution of the measure functional differential equation

$$
\left\{\begin{array}{l}
D y=f\left(y_{t}, t\right) D g, \quad t \in\left[t_{0}, t_{0}+\sigma\right] \\
y_{t_{0}}=\phi
\end{array}\right.
$$

Proof. The equality $y_{t_{0}}=\phi$ follows easily from the definitions of $y$ and $x\left(t_{0}\right)$. It remains to prove that for all $v \in\left[t_{0}, t_{0}+\sigma\right]$, we have

$$
y(v)-y\left(t_{0}\right)=\int_{t_{0}}^{v} f\left(y_{s}, s\right) \mathrm{d} g(s) .
$$

Let $v \in\left[t_{0}, t_{0}+\sigma\right]$. Using Lemma 4.4, we obtain

$$
y(v)-y\left(t_{0}\right)=x(v)(v)-x\left(t_{0}\right)\left(t_{0}\right)=x(v)(v)-x\left(t_{0}\right)(v)=\left(\int_{t_{0}}^{v} D F(x(\tau), t)\right)(v) .
$$

Thus,

$$
y(v)-y\left(t_{0}\right)-\int_{t_{0}}^{v} f\left(y_{s}, s\right) \mathrm{d} g(s)=\left(\int_{t_{0}}^{v} D F(x(\tau), t)\right)(v)-\int_{t_{0}}^{v} f\left(y_{s}, s\right) \mathrm{d} g(s) .
$$

Let an arbitrary $\varepsilon>0$ be given. Since $g$ is nondecreasing, it can have only a finite number of points $t \in\left[t_{0}, v\right]$ such that $\Delta^{+} g(t) \geq \varepsilon /(L+M)$. Denote these points by $t_{1}, \ldots, t_{m}$.

Consider a gauge $\delta:\left[t_{0}, t_{0}+\sigma\right] \rightarrow \mathbb{R}^{+}$such that

$$
\delta(\tau)<\min \left\{\frac{t_{k}-t_{k-1}}{2}, k=2, \ldots, m\right\}, \quad \tau \in\left[t_{0}, t_{0}+\sigma\right]
$$

and

$$
\delta(\tau)<\min \left\{\left|\tau-t_{k}\right|,\left|\tau-t_{k-1}\right| ; \tau \in\left(t_{k-1}, t_{k}\right) ; k=2, \ldots, m\right\}
$$


As in the proof of Theorem 4.5, these conditions ensure that if a point-interval pair $(\tau,[c, d])$ is $\delta$-fine, then $[c, d]$ contains at most one of the points $t_{1}, \ldots, t_{m}$, and, moreover, $\tau=t_{k}$ whenever $t_{k} \in[c, d]$.

Again, the gauge $\delta$ might be chosen in such a way that

$$
\int_{t_{k}}^{t_{k}+\delta\left(t_{k}\right)} L\left\|y_{s}-x\left(t_{k}\right)_{s}\right\|_{\infty} \mathrm{d} g(s)<\frac{\varepsilon}{2 m+1}, \quad k \in\{1, \ldots, m\} .
$$

According to Lemma 4.2, the function $F$ given by (4.2.3) belongs to the class $\mathcal{F}(O \times$ $\left.\left[t_{0}, t_{0}+\sigma\right], h\right)$, where

$$
h(t)=(L+M)\left[g(t)-g\left(t_{0}\right)\right], \quad t \in\left[t_{0}, t_{0}+\sigma\right] .
$$

Since

$$
|h(\tau+)-h(\tau)|=\left|(L+M) \Delta^{+} g(\tau)\right|<\varepsilon, \quad \tau \in\left[t_{0}, t_{0}+\sigma\right] \backslash\left\{t_{1}, \ldots, t_{m}\right\},
$$

we can assume that the gauge $\delta$ satisfies

$$
|h(\rho)-h(\tau)| \leq \varepsilon \quad \text { for every } \rho \in[\tau, \tau+\delta(\tau))
$$

Finally, the gauge $\delta$ should be such that

$$
\left\|\int_{t_{0}}^{v} D F(x(\tau), t)-\sum_{i=1}^{l}\left[F\left(x\left(\tau_{i}\right), s_{i}\right)-F\left(x\left(\tau_{i}\right), s_{i-1}\right)\right]\right\|<\varepsilon
$$

for every $\delta$-fine partition $\left\{\left(\tau_{i},\left[s_{i-1}, s_{i}\right]\right), i=1, \ldots, l\right\}$ of $\left[t_{0}, v\right]$. The existence of such a gauge follows from the definition of the Kurzweil integral.

Choose a particular $\delta$-fine partition $\left\{\left(\tau_{i},\left[s_{i-1}, s_{i}\right]\right), i=1, \ldots, l\right\}$ of $\left[t_{0}, v\right]$. By (4.2.12) and (4.2.13), we have

$$
\begin{gathered}
\left|y(v)-y\left(t_{0}\right)-\int_{t_{0}}^{v} f\left(y_{s}, s\right) \mathrm{d} g(s)\right|=\left|\left(\int_{t_{0}}^{v} D F(x(\tau), t)\right)(v)-\int_{t_{0}}^{v} f\left(y_{s}, s\right) \mathrm{d} g(s)\right|< \\
<\varepsilon+\left|\sum_{i=1}^{l}\left[F\left(x\left(\tau_{i}\right), s_{i}\right)-F\left(x\left(\tau_{i}\right), s_{i-1}\right)\right](v)-\int_{t_{0}}^{v} f\left(y_{s}, s\right) \mathrm{d} g(s)\right| \leq \\
\leq \varepsilon+\sum_{i=1}^{l}\left|\left[F\left(x\left(\tau_{i}\right), s_{i}\right)-F\left(x\left(\tau_{i}\right), s_{i-1}\right)\right](v)-\int_{s_{i-1}}^{s_{i}} f\left(y_{s}, s\right) \mathrm{d} g(s)\right| .
\end{gathered}
$$


The definition of $F$ yields

$$
\left[F\left(x\left(\tau_{i}\right), s_{i}\right)-F\left(x\left(\tau_{i}\right), s_{i-1}\right)\right](v)=\int_{s_{i-1}}^{s_{i}} f\left(x\left(\tau_{i}\right)_{s}, s\right) \mathrm{d} g(s),
$$

which implies

$$
\begin{gathered}
\left|\left[F\left(x\left(\tau_{i}\right), s_{i}\right)-F\left(x\left(\tau_{i}\right), s_{i-1}\right)\right](v)-\int_{s_{i-1}}^{s_{i}} f\left(y_{s}, s\right) \mathrm{d} g(s)\right|= \\
=\left|\int_{s_{i-1}}^{s_{i}} f\left(x\left(\tau_{i}\right)_{s}, s\right) \mathrm{d} g(s)-\int_{s_{i-1}}^{s_{i}} f\left(y_{s}, s\right) \mathrm{d} g(s)\right|=\left|\int_{s_{i-1}}^{s_{i}}\left[f\left(x\left(\tau_{i}\right)_{s}, s\right)-f\left(y_{s}, s\right)\right] \mathrm{d} g(s)\right| .
\end{gathered}
$$

By Lemma 4.4, for every $i \in\{1, \ldots, l\}$, we have $x\left(\tau_{i}\right)_{s}=x(s)_{s}=y_{s}$ for $s \in\left[s_{i-1}, \tau_{i}\right]$ and $y_{s}=x(s)_{s}=x\left(s_{i}\right)_{s}$ for $s \in\left[\tau_{i}, s_{i}\right]$. Therefore,

$$
\begin{aligned}
& \left|\int_{s_{i-1}}^{s_{i}}\left[f\left(x\left(\tau_{i}\right)_{s}, s\right)-f\left(y_{s}, s\right)\right] \mathrm{d} g(s)\right|=\left|\int_{\tau_{i}}^{s_{i}}\left[f\left(x\left(\tau_{i}\right)_{s}, s\right)-f\left(y_{s}, s\right)\right] \mathrm{d} g(s)\right|= \\
& =\left|\int_{\tau_{i}}^{s_{i}}\left[f\left(x\left(\tau_{i}\right)_{s}, s\right)-f\left(x\left(s_{i}\right)_{s}, s\right)\right] \mathrm{d} g(s)\right| \leq L \int_{\tau_{i}}^{s_{i}}\left\|x\left(\tau_{i}\right)_{s}-x\left(s_{i}\right)_{s}\right\|_{\infty} \mathrm{d} g(s),
\end{aligned}
$$

where the last inequality follows from condition $(\mathrm{C})$.

Again, we distinguish two cases:

(i) The intersection of $\left[s_{i-1}, s_{i}\right]$ and $\left\{t_{1}, \ldots, t_{m}\right\}$ contains a single point $t_{k}=\tau_{i}$.

(ii) The intersection of $\left[s_{i-1}, s_{i}\right]$ and $\left\{t_{1}, \ldots, t_{m}\right\}$ is empty.

In case (i), it follows from the definition of the gauge $\delta$ that

$$
L \int_{\tau_{i}}^{s_{i}}\left\|y_{s}-x\left(\tau_{i}\right)_{s}\right\|_{\infty} \mathrm{d} g(s) \leq \frac{\varepsilon}{2 m+1}
$$

that is,

$$
\left|\left[F\left(x\left(\tau_{i}\right), s_{i}\right)-F\left(x\left(\tau_{i}\right), s_{i-1}\right)\right](v)-\int_{s_{i-1}}^{s_{i}} f\left(y_{s}, s\right) \mathrm{d} g(s)\right| \leq \frac{\varepsilon}{2 m+1} .
$$

In case (ii), we use Lemma 2.15 to obtain the estimate

$$
\left\|x\left(s_{i}\right)_{s}-x\left(\tau_{i}\right)_{s}\right\|_{\infty} \leq\left\|x\left(s_{i}\right)-x\left(\tau_{i}\right)\right\|_{\infty} \leq h\left(s_{i}\right)-h\left(\tau_{i}\right) \leq \varepsilon,
$$


for every $s \in\left[\tau_{i}, s_{i}\right]$ and, thus,

$$
\left|\left[F\left(x\left(\tau_{i}\right), s_{i}\right)-F\left(x\left(\tau_{i}\right), s_{i-1}\right)\right](v)-\int_{s_{i-1}}^{s_{i}} f\left(y_{s}, s\right) \mathrm{d} g(s)\right| \leq \varepsilon L \int_{\tau_{i}}^{s_{i}} \mathrm{~d} g(s) .
$$

Combining cases (i) and (ii) and using the fact that case (i) occurs at most $2 m$ times, we obtain

$$
\begin{aligned}
& \sum_{i=1}^{l}\left|\left[F\left(x\left(\tau_{i}\right), s_{i}\right)-F\left(x\left(\tau_{i}\right), s_{i-1}\right)\right](v)-\int_{s_{i-1}}^{s_{i}} f\left(y_{s}, s\right) \mathrm{d} g(s)\right| \leq \\
& \leq \varepsilon \int_{t_{0}}^{t_{0}+\sigma} L \mathrm{~d} g(s)+\frac{2 m \varepsilon}{2 m+1}<\varepsilon\left(1+L\left(g\left(t_{0}+\sigma\right)-g\left(t_{0}\right)\right)\right) .
\end{aligned}
$$

Consequently,

$$
\left|y(v)-y\left(t_{0}\right)-\int_{t_{0}}^{v} f\left(y_{s}, s\right) \mathrm{d} g(s)\right|<\varepsilon\left(2+L\left(g\left(t_{0}+\sigma\right)-g\left(t_{0}\right)\right)\right),
$$

which completes the proof.

Remark 4.7. It follows from Lemma 4.4 that the relation

$$
y(\vartheta)= \begin{cases}x\left(t_{0}\right)(\vartheta), & t_{0}-r \leq \vartheta \leq t_{0} \\ x(\vartheta)(\vartheta), & t_{0} \leq \vartheta \leq t_{0}+\sigma\end{cases}
$$

found in the previous theorem can be replaced by a single equality

$$
y(\vartheta)=x\left(t_{0}+\sigma\right)(\vartheta)
$$

for $t_{0}-r \leq \vartheta \leq t_{0}+\sigma$.

Remark 4.8. Before proceeding further, we stop for a moment to discuss conditions (A), (B) and (C), which appear in the statements of Theorem 4.5 and Theorem 4.6.

Condition (A) requires the existence of the Kurzweil-Henstock-Stieltjes integral

$$
\int_{t_{0}}^{t_{0}+\sigma} f\left(y_{t}, t\right) \mathrm{d} g(t)
$$

for every $y \in O$. This class of integrable functions is quite large. For example, if $g(t)=t$, the Kurzweil-Henstock-Stieltjes integral reduces to the well-known Kurzweil-Henstock in- 
tegral, which generalizes both Lebesgue and Newton integrals (see [49], for instance). For a general nondecreasing function $g$, Theorem 2.9 provides a useful sufficient existence condition: the Kurzweil-Henstock-Stieltjes integral exists whenever $t \mapsto f\left(y_{t}, t\right)$ is a regulated function.

Conditions (B) and (C) can be replaced by weaker statements: an inspection of the proofs of Theorem 4.5 and Theorem 4.6 shows that it is enough to assume the existence of functions $M, L:\left[t_{0}, t_{0}+\sigma\right] \rightarrow \mathbb{R}$ which are Lebesgue integrable with respect to $g$ such that

$$
\left|\int_{a}^{b} f\left(y_{s}, s\right) \mathrm{d} g(s)\right| \leq \int_{a}^{b} M(s) \mathrm{d} g(s)
$$

and

$$
\left|\int_{a}^{b}\left[f\left(y_{s}, s\right)-f\left(z_{s}, s\right)\right] \mathrm{d} g(s)\right| \leq \int_{a}^{b} L(s)\left\|y_{s}-z_{s}\right\| \mathrm{d} g(s)
$$

for every $a, b \in\left[t_{0}, t_{0}+\sigma\right]$ and $y \in O$.

For a proof of this result with these conditions, the reader may consult the references [38] and [100]. Although we consider conditions (A), (B) and (C) in all results presented in the present work, it is possible to replace them by more general ones without significant modifications in the proofs.

\subsection{Measure FDEs and functional dynamic equations on time scales}

In this section, we prove that functional dynamic equations on time scales can be viewed as measure functional differential equations. The majority of the results presented in this section are new and are contained in [36].

Here, we use the same notation as in [98]. Given a real number $t \leq \sup \mathbb{T}$, let

$$
t^{*}=\inf \{s \in \mathbb{T} ; s \geq t\}
$$

Notice that $t^{*}$ might be different from $\sigma(t)$. The following example illustrates this fact.

Example 4.9. Let $\mathbb{T}=\mathbb{Z}$ and $t \in \mathbb{T}$, then

$$
t^{*}=\inf \{s \in \mathbb{Z} ; s \geq t\}=\inf \{t, t+1, t+2, \ldots\}=t
$$


and

$$
\sigma(t)=\inf \{s \in \mathbb{Z} ; s>t\}=\inf \{t+1, t+2, \ldots\}=t+1 .
$$

Thus $\sigma(t) \neq t^{*}$, for any $t \in \mathbb{T}=\mathbb{Z}$.

Coming back to time scales $\mathbb{T}$, we recall the reader that $\mathbb{T}$ is a closed set. Then, clearly, $t^{*} \in \mathbb{T}$. Let

$$
\mathbb{T}^{*}= \begin{cases}(-\infty, \sup \mathbb{T}], & \text { if } \sup \mathbb{T}<\infty \\ (-\infty, \infty), & \text { otherwise }\end{cases}
$$

Given a function $f: \mathbb{T} \rightarrow \mathbb{R}^{n}$, we consider its extension $f^{*}: \mathbb{T}^{*} \rightarrow \mathbb{R}^{n}$ defined by

$$
f^{*}(t)=f\left(t^{*}\right), \quad t \in \mathbb{T}^{*}
$$

Similarly, given an open set $O \subset X$ and a function $f: O \times \mathbb{T} \rightarrow \mathbb{R}^{n}$, we define

$$
f^{*}(x, t)=f\left(x, t^{*}\right), \quad t \in \mathbb{T}^{*}
$$

To understand better the definition above, see the next pictures. The first one illustrates the function $f: \mathbb{T} \rightarrow \mathbb{R}^{n}$ and the other one illustrates its extension $f^{*}: \mathbb{T}^{*} \rightarrow \mathbb{R}^{n}$. Notice that while the function $f$ is only defined in $\mathbb{T}$, its extension $f^{*}$ is defined in whole interval $(-\infty, \sup \mathbb{T}]$ if $\sup \mathbb{T}<\infty$ and in whole real line, otherwise.
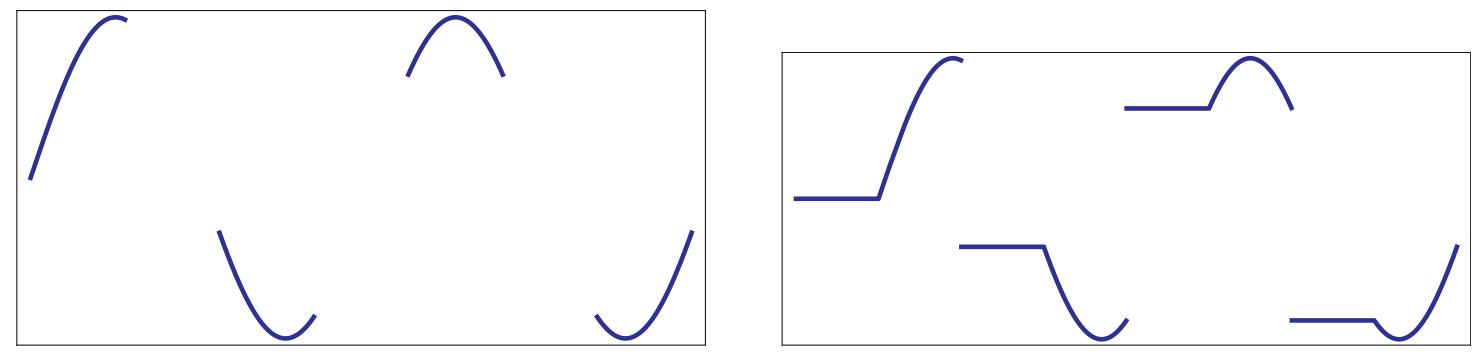

The next result can be found in [98], Lemma 4. It is a useful result, because it describes the extension $f^{*}$ based on the properties of $f$.

Lemma 4.10. If $f: \mathbb{T} \rightarrow \mathbb{R}^{n}$ is a regulated function, then $f^{*}: \mathbb{T}^{*} \rightarrow \mathbb{R}^{n}$ is also regulated. If $f$ is left-continuous on $\mathbb{T}$, then $f^{*}$ is left-continuous on $\mathbb{T}^{*}$. If $f$ is right-continuous on $\mathbb{T}$, then $f^{*}$ is right-continuous at right-dense points of $\mathbb{T}$.

Proof. At first, let us calculate $\lim _{t \rightarrow t_{0}^{-}} f^{*}(t)$, for $t_{0} \in \mathbb{T}^{*}$. If $t_{0} \in \mathbb{T}$ and $t_{0}$ is left-dense, 
then

$$
\lim _{t \rightarrow t_{0}^{-}} f^{*}(t)=\lim _{t \rightarrow t_{0}^{-}} f(t) .
$$

If $t_{0} \in \mathbb{T}$ and $t_{0}$ is left-scattered, then

$$
\lim _{t \rightarrow t_{0}^{-}} f^{*}(t)=f\left(t_{0}\right)=f^{*}\left(t_{0}\right)
$$

Finally, if $t_{0} \notin \mathbb{T}$, then

$$
\lim _{t \rightarrow t_{0}^{-}} f^{*}(t)=f\left(t_{0}^{*}\right)=f^{*}\left(t_{0}\right)
$$

Now, consider $\lim _{t \rightarrow t_{0}^{+}} f^{*}(t)$, where $t_{0} \in \mathbb{T}^{*}$ and $t_{0}<\sup \mathbb{T}^{*}$. If $t_{0} \in \mathbb{T}$ and it is right-dense, then

$$
\lim _{t \rightarrow t_{0}^{+}} f^{*}(t)=\lim _{t \rightarrow t_{0}^{+}} f(t) .
$$

If $t_{0} \in \mathbb{T}$ and it is right-scattered, then

$$
\lim _{t \rightarrow t_{0}^{+}} f^{*}(t)=f\left(\sigma\left(t_{0}\right)\right)
$$

Finally, if $t_{0} \notin \mathbb{T}$, then

$$
\lim _{t \rightarrow t_{0}^{+}} f^{*}(t)=f\left(t_{0}^{*}\right)=f^{*}\left(t_{0}\right)
$$

According to the following theorem, the $\Delta$-integral of a time scale function $f$ is in fact equivalent to the Kurzweil-Henstock-Stieltjes integral of the extended function $f^{*}$. It can be found in [98], Theorem 5. Since this result is a crucial result to prove the correspondences presented in this chapter, we will repeat its proof here.

Theorem 4.11. Let $f: \mathbb{T} \rightarrow \mathbb{R}^{n}$ be a rd-continuous function. Choose an arbitrary $a \in \mathbb{T}$ and define

$$
\begin{aligned}
& F_{1}(t)=\int_{a}^{t} f(s) \Delta s, \quad t \in \mathbb{T}, \\
& F_{2}(t)=\int_{a}^{t} f^{*}(s) \mathrm{d} g(s), \quad t \in \mathbb{T}^{*},
\end{aligned}
$$

where $g(s)=s^{*}$ for every $s \in \mathbb{T}^{*}$. Then $F_{2}=F_{1}^{*}$. In particular, $F_{2}(t)=F_{1}(t)$ for every $t \in \mathbb{T}$.

Proof. Note that the functions $F_{1}$ and $F_{2}$ are well-defined. In fact, it is clear that since $f$ is rd-continuous, the Riemann $\Delta$-integral in the definition of $F_{1}$ exists and also, since $f^{*}$ 
is regulated and $g$ is nondecreasing, the Kurzweil-Henstock-Stieltjes integral exists. To complete this proof, it is enough to prove the following two statements:

(1) $F_{1}(t)=F_{2}(t)$, for every $t \in \mathbb{T}$;

(2) If $t \in \mathbb{T}$ and $s=\sup \{u \in \mathbb{T} ; u<t\}$, then $F_{2}$ is constant on $[s, t)$.

At first, we will prove the second statement, since it is easier to prove: If $u, v \in(s, t]$ and $u<v$, then

$$
F_{2}(v)-F_{2}(u)=\int_{u}^{v} f^{*}(s) \mathrm{d} g(s)=0
$$

where the last equality follows from the definition of Kurzweil-Henstock-Stieltjes integral and the fact that $g$ is constant on $[u, v]$.

Now, let us prove the first statement. Note that, by definition, $F_{1}(a)=F_{2}(a)=0$ and, thus, it is sufficient to show that $F_{1}^{\Delta}(t)=F_{2}^{\Delta}(t)$ for every $t \in \mathbb{T}$, since any two functions with the same $\Delta$-derivatives differ only by a constant. By the properties of the Riemann $\Delta$-integral, $F_{1}{ }^{\Delta}(t)=f(t)$, and it remains to evaluate ${F_{2}}^{\Delta}$.

If $t$ is a right-dense point, then $f$ is continuous at $t$ and

$$
\lim _{s \rightarrow t} f^{*}(s)=f^{*}(t)=f(t)
$$

by Lemma 4.10. Therefore, given an arbitrary $\varepsilon>0$, there is a $\delta>0$ such that $\mid f^{*}(s)-$ $f(t) \mid<\varepsilon$ whenever $|s-t|<\delta$.

Now, consider a sequence of time scale points $\left\{t_{k}\right\}_{k=1}^{\infty}$ such that $\lim _{k \rightarrow \infty} t_{k}=t$. We can find a $k_{0} \in \mathbb{N}$ such that $\left|t_{k}-t\right|<\delta$ whenever $k \geq k_{0}$. Thus, for every $k \geq k_{0}$, we obtain

$$
\begin{gathered}
\left|\frac{F_{2}\left(t_{k}\right)-F_{2}(t)}{t_{k}-t}-f(t)\right|=\left|\frac{1}{t_{k}-t} \int_{t}^{t_{k}} f^{*}(s) \mathrm{d} g(s)-f(t)\right|= \\
=\left|\frac{1}{t_{k}-t} \int_{t}^{t_{k}}\left(f^{*}(s)-f(t)\right) \mathrm{d} g(s)\right|<\varepsilon \frac{g\left(t_{k}\right)-g(t)}{t_{k}-t}=\varepsilon
\end{gathered}
$$

since $g\left(t_{k}\right)=t_{k}$ and $g(t)=t$. It follows that

$$
\lim _{k \rightarrow \infty} \frac{F_{2}\left(t_{k}\right)-F_{2}(t)}{t_{k}-t}=f(t)
$$

that is, $F_{2}{ }^{\Delta}(t)=f(t)$.

On the other hand, when $t$ is a right-scattered point, we have

$$
F_{2}(\sigma(t))=F_{2}\left(t^{+}\right)=F_{2}(t)+f(t) \Delta^{+} g(t),
$$


where the first equality follows from the fact that $F_{2}$ is constant on $(t, \sigma(t)]$ and the second equality is a consequence of Corollary 2.7. But $\Delta^{+} g(t)=g\left(t^{+}\right)-g(t)=\sigma(t)-t=\mu(t)$, and it follows that

$$
F_{2}^{\Delta}(t)=\frac{F_{2}(\sigma(t))-F_{2}(t)}{\mu(t)}=f(t)
$$

and the result follows.

In what follows, we present a result which will be essential to prove our correspondence. It says that the Kurzweil-Henstock-Stieltjes integral $\int_{a}^{b} f^{*}(s) \mathrm{d} g(s)$ does not change if we replace $f^{*}$ by a different function which coincides with $f$ on $[a, b] \cap \mathbb{T}$. This is a new result and it is contained in [36].

Theorem 4.12. Let $\mathbb{T}$ be a time scale, $g(s)=s^{*}$ for every $s \in \mathbb{T}^{*},[a, b] \subset \mathbb{T}^{*}$. Consider a pair of functions $f_{1}, f_{2}:[a, b] \rightarrow \mathbb{R}^{n}$ such that $f_{1}(t)=f_{2}(t)$ for every $t \in[a, b] \cap \mathbb{T}$. If $\int_{a}^{b} f_{1}(t) \mathrm{d} g(t)$ exists, then $\int_{a}^{b} f_{2}(t) \mathrm{d} g(t)$ exists as well and both integrals have the same value.

Proof. Denote $I=\int_{a}^{b} f_{1}(s) \mathrm{d} g(s)$. Given an arbitrary $\varepsilon>0$, there is a gauge $\delta_{1}:[a, b] \rightarrow$ $\mathbb{R}^{+}$such that

$$
\left|\sum_{i=1}^{k} f_{1}\left(\tau_{i}\right)\left[g\left(s_{i}\right)-g\left(s_{i-1}\right)\right]-I\right|<\varepsilon,
$$

for every $\delta_{1}$-fine tagged partition $\left\{\left(\tau_{i},\left[s_{i-1}, s_{i}\right]\right), i=1, \ldots, k\right\}$.

Let

$$
\delta_{2}(t)= \begin{cases}\delta_{1}(t), & t \in[a, b] \cap \mathbb{T} \\ \min \left\{\delta_{1}(t), \frac{1}{2} \inf \{|t-s|, s \in \mathbb{T}\}\right\}, & t \in[a, b] \backslash \mathbb{T}\end{cases}
$$

Note that each $\delta_{2}$-fine tagged partition is also $\delta_{1}$-fine. Consider an arbitrary $\delta_{2}$-fine tagged partition $\left\{\left(\tau_{i},\left[s_{i-1}, s_{i}\right]\right), i=1, \ldots, k\right\}$.

For every $i \in\{1, \ldots, k\}$, there are two possibilities: either $\left[s_{i-1}, s_{i}\right] \cap \mathbb{T}=\emptyset$, or $\tau_{i} \in \mathbb{T}$. In the first case, $g\left(s_{i-1}\right)=g\left(s_{i}\right)$, and, therefore,

$$
f_{2}\left(\tau_{i}\right)\left[g\left(s_{i}\right)-g\left(s_{i-1}\right)\right]=0=f_{1}\left(\tau_{i}\right)\left[g\left(s_{i}\right)-g\left(s_{i-1}\right)\right] .
$$

In the second case, $f_{1}\left(\tau_{i}\right)=f_{2}\left(\tau_{i}\right)$ and

$$
f_{2}\left(\tau_{i}\right)\left[g\left(s_{i}\right)-g\left(s_{i-1}\right)\right]=f_{1}\left(\tau_{i}\right)\left[g\left(s_{i}\right)-g\left(s_{i-1}\right)\right] .
$$


Thus, we have

$$
\left|\sum_{i=1}^{k} f_{2}\left(\tau_{i}\right)\left[g\left(s_{i}\right)-g\left(s_{i-1}\right)\right]-I\right|=\left|\sum_{i=1}^{k} f_{1}\left(\tau_{i}\right)\left[g\left(s_{i}\right)-g\left(s_{i-1}\right)\right]-I\right|<\varepsilon
$$

Then, since $\varepsilon>0$ can be taken arbitrarily small, we conclude that $\int_{a}^{b} f_{2}(t) \mathrm{d} g(t)=I$.

We point out that when we are dealing with dynamic equations on time scales such that the $\Delta$-derivative of the unknown function $x: \mathbb{T} \rightarrow \mathbb{R}^{n}$ at $t \in \mathbb{T}$ depends on the values of $x(s)$, where $s \in[t-r, t] \cap \mathbb{T}$, we deal with one difficulty. Unlike the classical case, the function $x_{t}$ is now defined on a subset of $[-r, 0]$, and this subset may depend on $t$. We overcome this problem by considering the function $x_{t}^{*}$ instead. Throughout this and the following sections, $x_{t}^{*}$ stands for $\left(x^{*}\right)_{t}$. Clearly, $x_{t}^{*}$ contains the same information as $x_{t}$, but it is defined on the whole interval $[-r, 0]$. Thus, it seems reasonable to consider functional dynamic equations of the form

$$
x^{\Delta}(t)=f\left(x_{t}^{*}, t\right) .
$$

This equation is equivalent to a certain measure functional differential equation as it will be showed in the next new result.

The symbol $C\left([a, b]_{\mathbb{T}}, \mathbb{R}^{n}\right)$ will be used to denote the set of all continuous functions $f:[a, b]_{\mathbb{T}} \rightarrow \mathbb{R}^{n}$.

Theorem 4.13. Let $\left[t_{0}-r, t_{0}+\sigma\right]_{\mathbb{T}}$ be a time scale interval, $t_{0} \in \mathbb{T}, B \subset \mathbb{R}^{n}, C=$ $C\left(\left[t_{0}-r, t_{0}+\sigma\right]_{\mathbb{T}}, B\right), P=\left\{x_{t}^{*} ; x \in C, t \in\left[t_{0}, t_{0}+\sigma\right]\right\}, f: P \times\left[t_{0}, t_{0}+\sigma\right]_{\mathbb{T}} \rightarrow \mathbb{R}^{n}, \phi \in$ $C\left(\left[t_{0}-r, t_{0}\right]_{\mathbb{T}}, B\right)$. Assume that for every $x \in C$, the function $t \mapsto f\left(x_{t}^{*}, t\right)$ is rd-continuous on $\left[t_{0}, t_{0}+\sigma\right]_{\mathbb{T}}$. Define $g(s)=s^{*}$ for every $s \in\left[t_{0}, t_{0}+\sigma\right]$. If $x:\left[t_{0}-r, t_{0}+\sigma\right]_{\mathbb{T}} \rightarrow B$ is a solution of the functional dynamic equation

$$
\begin{aligned}
x^{\Delta}(t) & =f\left(x_{t}^{*}, t\right), \quad t \in\left[t_{0}, t_{0}+\sigma\right]_{\mathbb{T}}, \\
x(t) & =\phi(t), \quad t \in\left[t_{0}-r, t_{0}\right]_{\mathbb{T}},
\end{aligned}
$$

then $x^{*}:\left[t_{0}-r, t_{0}+\sigma\right] \rightarrow B$ satisfies

$$
\begin{aligned}
x^{*}(t) & =x^{*}\left(t_{0}\right)+\int_{t_{0}}^{t} f\left(x_{s}^{*}, s^{*}\right) \mathrm{d} g(s), \quad t \in\left[t_{0}, t_{0}+\sigma\right], \\
x_{t_{0}}^{*} & =\phi_{t_{0}}^{*} .
\end{aligned}
$$


Conversely, if $y:\left[t_{0}-r, t_{0}+\sigma\right] \rightarrow B$ is a solution of the following equation

$$
\begin{aligned}
y(t) & =y\left(t_{0}\right)+\int_{t_{0}}^{t} f\left(y_{s}, s^{*}\right) \mathrm{d} g(s), \quad t \in\left[t_{0}, t_{0}+\sigma\right], \\
y_{t_{0}} & =\phi_{t_{0}}^{*}
\end{aligned}
$$

then $y=x^{*}$, where $x:\left[t_{0}-r, t_{0}+\sigma\right]_{\mathbb{T}} \rightarrow B$, satisfies (4.3.1) and (4.3.2).

Proof. Assume that

$$
x^{\Delta}(t)=f\left(x_{t}^{*}, t\right), \quad t \in\left[t_{0}, t_{0}+\sigma\right]_{\mathbb{T}} .
$$

Then

$$
x(t)=x\left(t_{0}\right)+\int_{t_{0}}^{t} f\left(x_{s}^{*}, s\right) \Delta s, \quad t \in\left[t_{0}, t_{0}+\sigma\right]_{\mathbb{T}},
$$

and, by Theorem 4.11,

$$
x^{*}(t)=x^{*}\left(t_{0}\right)+\int_{t_{0}}^{t} f\left(x_{s^{*}}^{*}, s^{*}\right) \mathrm{d} g(s), \quad t \in\left[t_{0}, t_{0}+\sigma\right] .
$$

Since $f\left(x_{s^{*}}^{*}, s^{*}\right)=f\left(x_{s}^{*}, s^{*}\right)$ for every $s \in \mathbb{T}$, we can use Theorem 4.12 to conclude that

$$
x^{*}(t)=x^{*}\left(t_{0}\right)+\int_{t_{0}}^{t} f\left(x_{s}^{*}, s^{*}\right) \mathrm{d} g(s), \quad t \in\left[t_{0}, t_{0}+\sigma\right] .
$$

Conversely, assume that $y$ satisfies

$$
y(t)=y\left(t_{0}\right)+\int_{t_{0}}^{t} f\left(y_{s}, s^{*}\right) \mathrm{d} g(s), \quad t \in\left[t_{0}, t_{0}+\sigma\right] .
$$

Note that $g$ is constant on every interval $(\alpha, \beta]$, where $\beta \in \mathbb{T}$ and $\alpha=\sup \{\tau \in \mathbb{T} ; \tau<\beta\}$. Thus $y$ has the same property and it follows that $y=x^{*}$ for some $x:\left[t_{0}-r, t_{0}+\sigma\right]_{\mathbb{T}} \rightarrow B$. Using Corollary 2.7, it is easy to see that $x$ is continuous on $\left[t_{0}-r, t_{0}+\sigma\right]_{\mathbb{T}}$. By reversing our previous reasoning, we conclude that $x$ satisfies (4.3.1) and (4.3.2).

Example 4.14. There is a fairly large number of papers devoted to delay dynamic equations of the form

$$
x^{\Delta}(t)=h\left(t, x(t), x\left(\tau_{1}(t)\right), \ldots, x\left(\tau_{k}(t)\right)\right),
$$

where $\tau_{i}: \mathbb{T} \rightarrow \mathbb{T}$ are functions corresponding to the delays, that is $\tau_{i}(t) \leq t$ for every $t \in \mathbb{T}$ and $i=1, \ldots, k$. Also, consider the function $t \mapsto h\left(t, x(t), x\left(\tau_{1}(t)\right), \ldots, x\left(\tau_{k}(t)\right)\right)$ is rd-continuous, whenever $x$ is a continuous function. 
Suppose the delays are bounded, that is, there exists a constant $r>0$ such that $t-r \leq \tau_{i}(t) \leq t$, or equivalently $-r \leq \tau_{i}(t)-t \leq 0$. Then, it is possible to rewrite (4.3.3) in the form

$$
x^{\Delta}(t)=f\left(x_{t}^{*}, t\right)
$$

by taking

$$
f(y, t)=h\left(t, y(0), y\left(\tau_{1}(t)-t\right), \ldots, y\left(\tau_{k}(t)-t\right)\right)
$$

for every $y:[-r, 0] \rightarrow \mathbb{R}^{n}$. Also, if $x$ is continuous on $\left[t_{0}-r, t_{0}+\sigma\right]_{\mathbb{T}}$, then the function $t \mapsto f\left(x_{t}^{*}, t\right)$ is rd-continuous on $\left[t_{0}, t_{0}+\sigma\right]_{\mathbb{T}}$.

An important special case is represented by linear delay dynamic equations of the form

$$
x^{\Delta}(t)=\sum_{i=1}^{k} p_{i}(t) x\left(\tau_{i}(t)\right)+q(t)
$$

where $q, p_{1}, \ldots, p_{k}$ and $\tau_{1}, \ldots, \tau_{k}$ are rd-continuous functions on $\left[t_{0}, t_{0}+\sigma\right]_{\mathbb{T}}$. The corresponding functional dynamic equation is

$$
x^{\Delta}(t)=f\left(x_{t}^{*}, t\right)
$$

where

$$
f(y, t)=\sum_{i=1}^{k} p_{i}(t) y\left(\tau_{i}(t)-t\right)+q(t)
$$

for every $y:[-r, 0] \rightarrow \mathbb{R}^{n}$. Again, we see that $t \mapsto f\left(x_{t}^{*}, t\right)$ is rd-continuous on $\left[t_{0}, t_{0}+\sigma\right]_{\mathbb{T}}$ whenever $x$ is continuous on $\left[t_{0}-r, t_{0}+\sigma\right]_{\mathbb{T}}$. Moreover, for each pair of functions $y, z$ : $[-r, 0] \rightarrow \mathbb{R}^{n}$, we have

$$
\begin{aligned}
\mid f(y, t) & -f(z, t)\left|\leq \sum_{i=1}^{k}\right| p_{i}(t)\left(y\left(\tau_{i}(t)-t\right)-z\left(\tau_{i}(t)-t\right)\right) \mid \\
& \leq\left(\sum_{i=1}^{k}\left|p_{i}(t)\right|\right)\|y-z\|_{\infty} \leq L\|y-z\|_{\infty}
\end{aligned}
$$

with

$$
L=\sup _{t \in\left[t_{0}, t_{0}+\sigma\right]_{\mathbb{T}}} \sum_{i=1}^{k}\left|p_{i}(t)\right|,
$$

that is, $f$ is Lipschitz-continuous in the first variable. 


\subsection{Impulsive measure FDEs and measure FDEs}

In this section, we present the impulsive measure functional differential equations. Also, we describe the relation between them and measure functional differential equations. All the results presented in this section are original and they are contained in [37].

As we mentioned earlier, an initial value problem for a measure functional differential equation can be described in the form

$$
\left\{\begin{array}{l}
D x=f\left(x_{s}, s\right) D g(s), \quad t \in\left[t_{0}, t_{0}+\sigma\right] \\
x_{t_{0}}=\phi
\end{array}\right.
$$

where $D x$ and $D g$ are the distributional derivatives, in the sense of L. Schwartz, taken with respect to $x$ and $g$ respectively. Its integral form is given by

$$
\begin{aligned}
x(t) & =x\left(t_{0}\right)+\int_{t_{0}}^{t} f\left(x_{s}, s\right) \mathrm{d} g(s), \quad t \in\left[t_{0}, t_{0}+\sigma\right], \\
x_{t_{0}} & =\phi,
\end{aligned}
$$

where the Kurzweil-Henstock-Stieltjes integral on the right-hand side is taken with respect to a nondecreasing function $g:\left[t_{0}, t_{0}+\sigma\right] \rightarrow \mathbb{R}$. These equations have been studied in [19], [36], among others papers.

Let $O \subset G\left(\left[t_{0}-r, t_{0}+\sigma\right], \mathbb{R}^{n}\right)$ be open and $P=\left\{y_{t}: y \in O, \quad t \in\left[t_{0}, t_{0}+\sigma\right]\right\}$ be a subset of $G\left([-r, 0], \mathbb{R}^{n}\right)$.

Consider the impulsive measure functional differential equation given by

$$
\begin{aligned}
x(v)-x(u) & =\int_{u}^{v} f\left(x_{s}, s\right) \mathrm{d} g(s), \text { whenever } u, v \in J_{k} \text { for some } k \in\{0, \ldots, m\}, \\
\Delta^{+} x\left(t_{k}\right) & =I_{k}\left(x\left(t_{k}\right)\right), \quad k \in\{1, \ldots, m\}, \\
x_{t_{0}} & =\phi
\end{aligned}
$$

where $g$ is a left continuous function and the moments of impulses $t_{1}, \ldots, t_{m}$ are preassigned, where $t_{0} \leq t_{1}<\cdots<t_{m}<t_{0}+\sigma$ and $J_{0}=\left[t_{0}, t_{1}\right], J_{k}=\left(t_{k}, t_{k+1}\right]$ for $k \in\{1, \ldots, m-1\}$, and $J_{m}=\left(t_{m}, t_{0}+\sigma\right]$. Also, for every $k \in\{1, \ldots, m\}$, let the impulse at $t_{k}$ be described by the operator $I_{k}: \mathbb{R}^{n} \rightarrow \mathbb{R}^{n}$. In other words, suppose the solution $x$ satisfies $\Delta^{+} x\left(t_{k}\right)=I_{k}\left(x\left(t_{k}\right)\right)$.

Also, notice that the value of the integral $\int_{u}^{v} f\left(x_{s}, s\right) \mathrm{d} g(s)$, where $u, v \in J_{k}$, does not change if we replace $g$ by a function $\tilde{g}$ such that $g-\tilde{g}$ is a constant function on $J_{k}$ 
(this follows easily from the definition of the Kurzweil-Henstock-Stieltjes integral). Thus, without loss of generality, we can assume that $g$ is such that $\Delta^{+} g\left(t_{k}\right)=0$ for every $k \in\{1, \ldots, m\}$. Since $g$ is a left-continuous function, it follows that $g$ is continuous at $t_{1}, \ldots, t_{m}$. Under this assumption, our problem can be rewritten as

$$
\begin{aligned}
x(t) & =x\left(t_{0}\right)+\int_{t_{0}}^{t} f\left(x_{s}, s\right) \mathrm{d} g(s)+\sum_{\substack{k \in\{1, \ldots, m\}, t_{k}<t}} I_{k}\left(x\left(t_{k}\right)\right), \quad t \in\left[t_{0}, t_{0}+\sigma\right], \\
x_{t_{0}} & =\phi .
\end{aligned}
$$

Indeed, the function $t \mapsto \int_{t_{0}}^{t} f\left(x_{s}, s\right) \mathrm{d} g(s)$ is continuous at $t_{1}, \ldots, t_{m}$ (see Corollary 2.7) and, therefore, $\Delta^{+} x\left(t_{k}\right)=I_{k}\left(x\left(t_{k}\right)\right)$ for every $k \in\{1, \ldots, m\}$.

Alternatively, the sum on the right-hand side of (4.4.1) might be rewritten in the form $\sum_{k=1}^{m} I_{k}\left(x\left(t_{k}\right)\right) H_{t_{k}}(t)$, where $H_{v}$ denotes the characteristic function of $(v, \infty)$.

In the sequel, we start by presenting an important result for the Kurzweil-HenstockStieltjes-integral. This result will be essential to prove the correspondence between measure functional differential equations and impulsive measure functional differential equations.

Lemma 4.15. Let $m \in \mathbb{N}, a \leq t_{1}<t_{2}<\cdots<t_{m} \leq b$. Consider a pair of functions $f:[a, b] \rightarrow \mathbb{R}$ and $g:[a, b] \rightarrow \mathbb{R}$, where $g$ is regulated, left continuous on $[a, b]$, and continuous at $t_{1}, \ldots, t_{m}$. Let $\tilde{f}:[a, b] \rightarrow \mathbb{R}$ and $\tilde{g}:[a, b] \rightarrow \mathbb{R}$ be such that $\tilde{f}(t)=f(t)$ for every $t \in[a, b] \backslash\left\{t_{1}, \ldots, t_{m}\right\}$ and $\tilde{g}-g$ is constant on each of the intervals $\left[a, t_{1}\right]$, $\left(t_{1}, t_{2}\right], \ldots,\left(t_{m-1}, t_{m}\right],\left(t_{m}, b\right]$. Then the integral $\int_{a}^{b} \tilde{f}(t) \mathrm{d} \tilde{g}(t)$ exists, if and only if, the integral $\int_{a}^{b} f(t) \mathrm{d} g(t)$ exists. In this case, we have

$$
\int_{a}^{b} \tilde{f}(t) \mathrm{d} \tilde{g}(t)=\int_{a}^{b} f(t) \mathrm{d} g(t)+\sum_{\substack{k \in\{1, \ldots, m\} \\ t_{k}<b}} \tilde{f}\left(t_{k}\right) \Delta^{+} \tilde{g}\left(t_{k}\right)
$$

Proof. Using the definition of the Kurzweil-Henstock-Stieltjes integral, we obtain

$$
\int_{a}^{t_{1}} \tilde{f}(t) \mathrm{d}(\tilde{g}-g)(t)=0
$$

It follows from Corollary 2.5 and the definition of Kurzweil-Henstock-Stieltjes integral that

$\int_{t_{k}}^{t_{k+1}} \tilde{f}(t) \mathrm{d}(\tilde{g}-g)(t)=\lim _{\tau \rightarrow t_{k}+} \int_{\tau}^{t_{k+1}} \tilde{f}(t) \mathrm{d}(\tilde{g}-g)(t)+\tilde{f}\left(t_{k}\right) \Delta^{+}(\tilde{g}-g)\left(t_{k}\right)=\tilde{f}\left(t_{k}\right) \Delta^{+} \tilde{g}\left(t_{k}\right)$, 
for every $k \in\{1, \ldots, m-1\}$. If $t_{m}=b$, then $\int_{t_{m}}^{b} \tilde{f}(t) \mathrm{d}(\tilde{g}-g)(t)=0$. Otherwise,

$$
\int_{t_{m}}^{b} \tilde{f}(t) \mathrm{d}(\tilde{g}-g)(t)=\lim _{\tau \rightarrow t_{m}+} \int_{\tau}^{b} \tilde{f}(t) \mathrm{d}(\tilde{g}-g)(t)+\tilde{f}\left(t_{m}\right) \Delta^{+}(\tilde{g}-g)\left(t_{m}\right)=\tilde{f}\left(t_{m}\right) \Delta^{+} \tilde{g}\left(t_{m}\right) .
$$

Consequently, $\int_{a}^{b} \tilde{f}(t) \mathrm{d}(\tilde{g}-g)(t)$ exists and

$$
\int_{a}^{b} \tilde{f}(t) \mathrm{d}(\tilde{g}-g)(t)=\sum_{\substack{k \in\{1, \ldots, m\} \\ t_{k}<b}} \tilde{f}\left(t_{k}\right) \Delta^{+} \tilde{g}\left(t_{k}\right)
$$

By Corollaries 2.5 and 2.7, we have

$$
\begin{gathered}
\int_{a}^{t_{1}} \tilde{f}(t) \mathrm{d} g(t)=\lim _{\tau \rightarrow t_{1}-} \int_{a}^{\tau} \tilde{f}(t) \mathrm{d} g(t)=\lim _{\tau \rightarrow t_{1}-} \int_{a}^{\tau} f(t) \mathrm{d} g(t)=\int_{a}^{t_{1}} f(t) \mathrm{d} g(t), \\
\int_{t_{k}}^{t_{k+1}} \tilde{f}(t) \mathrm{d} g(t)=\lim _{\substack{\sigma \rightarrow t_{k}+, \tau \rightarrow t_{k+1}-}} \int_{\sigma}^{\tau} \tilde{f}(t) \mathrm{d} g(t)=\lim _{\substack{\sigma \rightarrow t_{k}+, \tau \rightarrow t_{k+1}-}} \int_{\sigma}^{\tau} f(t) \mathrm{d} g(t)=\int_{t_{k}}^{t_{k+1}} f(t) \mathrm{d} g(t),
\end{gathered}
$$

for $k \in\{1, \ldots, m-1\}$ and

$$
\int_{t_{m}}^{b} \tilde{f}(t) \mathrm{d} g(t)=\lim _{\tau \rightarrow t_{m}+} \int_{\tau}^{b} \tilde{f}(t) \mathrm{d} g(t)=\lim _{\tau \rightarrow t_{m}+} \int_{\tau}^{b} f(t) \mathrm{d} g(t)=\int_{t_{m}}^{b} f(t) \mathrm{d} g(t) .
$$

These three relations might be read not only from left to right, but also from right to left. In other words, the integrals on the left-hand sides exist, if and only if, the integrals on the right-hand sides exist. Combining these three relations, it follows that $\int_{a}^{b} \tilde{f}(t) \mathrm{d} g(t)$ exists, if and only if, $\int_{a}^{b} f(t) \mathrm{d} g(t)$ exists. In this case, their values are equal.

To conclude the proof, it is enough to notice that

$$
\int_{a}^{b} \tilde{f}(t) \mathrm{d} \tilde{g}(t)=\int_{a}^{b} \tilde{f}(t) \mathrm{d} g(t)+\int_{a}^{b} \tilde{f}(t) \mathrm{d}(\tilde{g}-g)(t)=\int_{a}^{b} f(t) \mathrm{d} g(t)+\sum_{\substack{k \in\{1, \ldots, m\}, t_{k}<b}} \tilde{f}\left(t_{k}\right) \Delta^{+} \tilde{g}\left(t_{k}\right) .
$$

The following theorem shows that impulsive measure functional differential equations of the form (4.4.1) can always be transformed into measure functional differential equations.

Theorem 4.16. Let $m \in \mathbb{N}, t_{0} \leq t_{1}<\cdots<t_{m}<t_{0}+\sigma, B \subset \mathbb{R}^{n}, I_{1}, \ldots, I_{m}: B \rightarrow \mathbb{R}^{n}$, $P=G([-r, 0], B), f: P \times\left[t_{0}, t_{0}+\sigma\right] \rightarrow \mathbb{R}^{n}$. Assume that $g:\left[t_{0}, t_{0}+\sigma\right] \rightarrow \mathbb{R}$ is a regulated 
left-continuous function which is continuous at $t_{1}, \ldots, t_{m}$. For every $y \in P$, define

$$
\tilde{f}(y, t)= \begin{cases}f(y, t), & t \in\left[t_{0}, t_{0}+\sigma\right] \backslash\left\{t_{1}, \ldots, t_{m}\right\}, \\ I_{k}(y(0)), & t=t_{k} \text { for some } k \in\{1, \ldots, m\} .\end{cases}
$$

Moreover, let $c_{1}, \ldots, c_{m} \in \mathbb{R}$ be constants such that the function $\tilde{g}:\left[t_{0}, t_{0}+\sigma\right] \rightarrow \mathbb{R}$ given by

$$
\tilde{g}(t)= \begin{cases}g(t), & t \in\left[t_{0}, t_{1}\right], \\ g(t)+c_{k}, & t \in\left(t_{k}, t_{k+1}\right] \text { for some } k \in\{1, \ldots, m-1\}, \\ g(t)+c_{m}, & t \in\left(t_{m}, t_{0}+\sigma\right]\end{cases}
$$

satisfies $\Delta^{+} \tilde{g}\left(t_{k}\right)=1$ for every $k \in\{1, \ldots, m\}$. Then, $x \in G\left(\left[t_{0}-r, t_{0}+\sigma\right], B\right)$ is a solution of the problem

$$
\left\{\begin{aligned}
x(t) & =x\left(t_{0}\right)+\int_{t_{0}}^{t} f\left(x_{s}, s\right) \mathrm{d} g(s)+\sum_{\substack{k \in\{1, \ldots, m\}, t_{k}<t}} I_{k}\left(x\left(t_{k}\right)\right), \quad t \in\left[t_{0}, t_{0}+\sigma\right], \\
x_{t_{0}} & =\phi,
\end{aligned}\right.
$$

if and only if, $x$ is also a solution of the problem

$$
\left\{\begin{array}{l}
x(t)=x\left(t_{0}\right)+\int_{t_{0}}^{t} \tilde{f}\left(x_{s}, s\right) \mathrm{d} \tilde{g}(s), \quad t \in\left[t_{0}, t_{0}+\sigma\right] \\
x_{t_{0}}=\phi
\end{array}\right.
$$

Proof. According to Lemma 4.15, we have

$$
\begin{gathered}
\int_{t_{0}}^{t} \tilde{f}\left(x_{s}, s\right) \mathrm{d} \tilde{g}(s)=\int_{t_{0}}^{t} f\left(x_{s}, s\right) \mathrm{d} g(s)+\sum_{\substack{k \in\{1, \ldots, m\}, t_{k}<t}} \tilde{f}\left(x_{t_{k}}, t_{k}\right) \Delta^{+} \tilde{g}\left(t_{k}\right) \\
=\int_{t_{0}}^{t} f\left(x_{s}, s\right) \mathrm{d} g(s)+\sum_{\substack{k \in\{1, \ldots, m\}, t_{k}<t}} I_{k}\left(x\left(t_{k}\right)\right),
\end{gathered}
$$

that is, the right-hand sides of (4.4.2) and (4.4.3) are indeed identical.

Remark 4.17. When $g(t)=t$ for every $t \in\left[t_{0}, t_{0}+\sigma\right]$, equation (4.4.2) reduces to the usual type of impulsive functional differential equation

$$
x(t)=x\left(t_{0}\right)+\int_{t_{0}}^{t} f\left(x_{s}, s\right) \mathrm{d} s+\sum_{\substack{k \in\{1, \ldots, m\} \\ t_{k}<t}} I_{k}\left(x\left(t_{k}\right)\right), \quad t \in\left[t_{0}, t_{0}+\sigma\right] .
$$


Basic results concerning this type of equations were obtained by M. Federson and Š. Schwabik in [40]; the main tool in their investigations was the theory of generalized ordinary differential equations in a Banach space. The previous theorem suggests a different approach: impulsive measure functional differential equations are a special case of measure functional differential equations and, therefore, the existing theory of measure equations can be used in the study of impulsive equations.

The next lemma describes how the Carathéodory and Lipschitz-type conditions concerning the function $f$ and the Lipschitz and boundedness conditions for the impulse operators can be transferred to $\tilde{f}$, when it is defined in Theorem 4.16.

Lemma 4.18. Let $m \in \mathbb{N}, t_{0} \leq t_{1}<\cdots<t_{m}<t_{0}+\sigma, B \subset \mathbb{R}^{n}, I_{1}, \ldots, I_{m}: B \rightarrow \mathbb{R}^{n}, P=$ $G([-r, 0], B), O=G\left(\left[t_{0}-r, t_{0}+\sigma\right], B\right)$. Assume that $g:\left[t_{0}, t_{0}+\sigma\right] \rightarrow \mathbb{R}$ is a left-continuous nondecreasing function which is continuous at $t_{1}, \ldots, t_{m}$. Let $f: P \times\left[t_{0}, t_{0}+\sigma\right] \rightarrow \mathbb{R}^{n}$ be a function such that the integral $\int_{t_{0}}^{t_{0}+\sigma} f\left(y_{t}, t\right) \mathrm{d} g(t)$ exists for every $y \in O$. For every $y \in P$, define

$$
\tilde{f}(y, t)= \begin{cases}f(y, t), & t \in\left[t_{0}, t_{0}+\sigma\right] \backslash\left\{t_{1}, \ldots, t_{m}\right\} \\ I_{k}(y(0)), & t=t_{k} \text { for some } k \in\{1, \ldots, m\}\end{cases}
$$

Moreover, let $c_{1}, \ldots, c_{m} \in \mathbb{R}$ be constants such that the function $\tilde{g}:\left[t_{0}, t_{0}+\sigma\right] \rightarrow \mathbb{R}$ given by

$$
\tilde{g}(t)= \begin{cases}g(t), & t \in\left[t_{0}, t_{1}\right], \\ g(t)+c_{k}, & t \in\left(t_{k}, t_{k+1}\right] \text { for some } k \in\{1, \ldots, m-1\}, \\ g(t)+c_{m}, & t \in\left(t_{m}, t_{0}+\sigma\right]\end{cases}
$$

satisfies $\Delta^{+} \tilde{g}\left(t_{k}\right)=1$ for every $k \in\{1, \ldots, m\}$. Then the following assertions hold:

1. Assume there exist a constant $M_{1}>0$ such that

$$
\left|\int_{u_{1}}^{u_{2}} f\left(y_{t}, t\right) \mathrm{d} g(t)\right| \leq M_{1}\left[g\left(u_{2}\right)-g\left(u_{1}\right)\right]
$$

whenever $t_{0} \leq u_{1} \leq u_{2} \leq t_{0}+\sigma, y \in O$, and a constant $M_{2}>0$ such that

$$
\left|I_{k}(x)\right| \leq M_{2}
$$


for every $k \in\{1, \ldots, m\}$ and $x \in B$. Then

$$
\left|\int_{u_{1}}^{u_{2}} \tilde{f}\left(y_{t}, t\right) \mathrm{d} \tilde{g}(t)\right| \leq\left(M_{1}+M_{2}\right)\left(\tilde{g}\left(u_{2}\right)-\tilde{g}\left(u_{1}\right)\right)
$$

whenever $t_{0} \leq u_{1} \leq u_{2} \leq t_{0}+\sigma$ and $y \in O$.

2. Assume there exists a constant $L_{1}>0$ such that

$$
\left|\int_{u_{1}}^{u_{2}}\left[f\left(y_{t}, t\right)-f\left(z_{t}, t\right)\right] \mathrm{d} g(t)\right| \leq L_{1} \int_{u_{1}}^{u_{2}}\left\|y_{t}-z_{t}\right\|_{\infty} \mathrm{d} g(t)
$$

whenever $t_{0} \leq u_{1} \leq u_{2} \leq t_{0}+\sigma, y, z \in O$, and a constant $L_{2}>0$ such that

$$
\left|I_{k}(x)-I_{k}(y)\right| \leq L_{2}\|x-y\|
$$

for every $k \in\{1, \ldots, m\}$ and $x, y \in B$. Then

$$
\left|\int_{u_{1}}^{u_{2}}\left[\tilde{f}\left(y_{t}, t\right)-\tilde{f}\left(z_{t}, t\right)\right] \mathrm{d} \tilde{g}(t)\right| \leq\left(L_{1}+L_{2}\right) \int_{u_{1}}^{u_{2}}\left\|y_{t}-z_{t}\right\|_{\infty} \mathrm{d} \tilde{g}(t)
$$

whenever $t_{0} \leq u_{1} \leq u_{2} \leq t_{0}+\sigma$ and $y, z \in O$.

Proof. Since $g$ is nondecreasing, it follows that $\tilde{g}$ is also nondecreasing. Moreover, we have

$$
\tilde{g}(v)-\tilde{g}(u) \geq g(v)-g(u)
$$

whenever $t_{0} \leq u \leq v \leq t_{0}+\sigma$.

In order to prove the first statement, let $t_{0} \leq u_{1} \leq u_{2} \leq t_{0}+\sigma$ and $y \in O$. From Lemma 4.15, we obtain

$$
\int_{u_{1}}^{u_{2}} \tilde{f}\left(y_{t}, t\right) \mathrm{d} \tilde{g}(t)=\int_{u_{1}}^{u_{2}} f\left(y_{t}, t\right) \mathrm{d} g(t)+\sum_{\substack{k \in\{1, \ldots, m\}, u_{1} \leq t_{k}<u_{2}}} I_{k}\left(y\left(t_{k}\right)\right) \Delta^{+} \tilde{g}\left(t_{k}\right)
$$

and therefore

$$
\begin{gathered}
\quad\left|\int_{u_{1}}^{u_{2}} \tilde{f}\left(y_{t}, t\right) \mathrm{d} \tilde{g}(t)\right| \leq M_{1}\left[g\left(u_{2}\right)-g\left(u_{1}\right)\right]+M_{2} \sum_{\substack{k \in\{1, \ldots, m\}, u_{1} \leq t_{k}<u_{2}}} \Delta^{+} \tilde{g}\left(t_{k}\right) \leq \\
\leq M_{1}\left[\tilde{g}\left(u_{2}\right)-\tilde{g}\left(u_{1}\right)\right]+M_{2}\left[\tilde{g}\left(u_{2}\right)-\tilde{g}\left(u_{1}\right)\right]=\left(M_{1}+M_{2}\right)\left[\tilde{g}\left(u_{2}\right)-\tilde{g}\left(u_{1}\right)\right] .
\end{gathered}
$$


To prove the second statement, let $t_{0} \leq u_{1} \leq u_{2} \leq t_{0}+\sigma$ and $y, z \in O$. Using Lemma 4.15 again, we obtain

$$
\begin{gathered}
\int_{u_{1}}^{u_{2}}\left[\tilde{f}\left(y_{t}, t\right)-\tilde{f}\left(z_{t}, t\right)\right] \mathrm{d} \tilde{g}(t)=\int_{u_{1}}^{u_{2}}\left[f\left(y_{t}, t\right)-f\left(z_{t}, t\right)\right] \mathrm{d} g(t)+ \\
+\sum_{\substack{k \in\{1, \ldots, m\}, u_{1} \leq t_{k}<u_{2}}}\left[I_{k}\left(y\left(t_{k}\right)\right)-I_{k}\left(z\left(t_{k}\right)\right)\right] \Delta^{+} \tilde{g}\left(t_{k}\right) .
\end{gathered}
$$

Consequently,

$$
\left|\int_{u_{1}}^{u_{2}}\left[\tilde{f}\left(y_{t}, t\right)-\tilde{f}\left(z_{t}, t\right)\right] \mathrm{d} \tilde{g}(t)\right| \leq L_{1} \int_{u_{1}}^{u_{2}}\left\|y_{t}-z_{t}\right\|_{\infty} \mathrm{d} g(t)+L_{2} \sum_{\substack{k \in\{1, \ldots, m\}, u_{1} \leq t_{k}<u_{2}}}\left|y\left(t_{k}\right)-z\left(t_{k}\right)\right| \Delta^{+} \tilde{g}\left(t_{k}\right) .
$$

Using equation (4.4.4) and the definition of Kurzweil-Henstock-Stieltjes integral, we obtain

$$
\int_{u_{1}}^{u_{2}}\left\|y_{t}-z_{t}\right\|_{\infty} \mathrm{d} g(t) \leq \int_{u_{1}}^{u_{2}}\left\|y_{t}-z_{t}\right\|_{\infty} \mathrm{d} \tilde{g}(t)
$$

Now, we observe that the function

$$
h(s)=\int_{t_{0}}^{s}\left\|y_{t}-z_{t}\right\|_{\infty} \mathrm{d} \tilde{g}(t), \quad s \in\left[t_{0}, t_{0}+\sigma\right],
$$

is nondecreasing and $\Delta^{+} h\left(t_{k}\right)=\left\|y_{t_{k}}-z_{t_{k}}\right\|_{\infty} \Delta^{+} \tilde{g}\left(t_{k}\right)$ for $k \in\{1, \ldots, m\}$. Therefore

$$
\begin{aligned}
& L_{2} \sum_{\substack{k \in\{1, \ldots, m\}, u_{1} \leq t_{k}<u_{2}}}\left|y\left(t_{k}\right)-z\left(t_{k}\right)\right| \Delta^{+} \tilde{g}\left(t_{k}\right) \leq L_{2} \sum_{\substack{k \in\{1, \ldots, m\}, u_{1} \leq t_{k}<u_{2}}}\left\|y_{t_{k}}-z_{t_{k}}\right\|_{\infty} \Delta^{+} \tilde{g}\left(t_{k}\right) \\
& =L_{2} \sum_{\substack{k \in\{1, \ldots, m\}, u_{1} \leq t_{k}<u_{2}}} \Delta^{+} h\left(t_{k}\right) \leq L_{2}\left(h\left(u_{2}\right)-h\left(u_{1}\right)\right)=L_{2} \int_{u_{1}}^{u_{2}}\left\|y_{t}-z_{t}\right\|_{\infty} \mathrm{d} \tilde{g}(t),
\end{aligned}
$$

and it follows that

$$
\left|\int_{u_{1}}^{u_{2}}\left[\tilde{f}\left(y_{t}, t\right)-\tilde{f}\left(z_{t}, t\right)\right] \mathrm{d} \tilde{g}(t)\right| \leq\left(L_{1}+L_{2}\right) \int_{u_{1}}^{u_{2}}\left\|y_{t}-z_{t}\right\|_{\infty} \mathrm{d} \tilde{g}(t) .
$$




\subsection{Impulsive functional differential and dynamic equa- tions on time scales}

In this section, we present a correspondence between the solutions of an impulsive measure FDE and the solutions of an impulsive functional dynamic equation on time scales. All the results presented here are original and they are contained in [37].

We start by presenting a result which will be essential to obtain a generalization of Theorem 4.11 in the sequel. Such result is original and can be found in [37], Theorem 4.5.

Theorem 4.19. Let $a, b \in \mathbb{T}$ and $f:[a, b]_{\mathbb{T}} \rightarrow \mathbb{R}^{n}$ be an arbitrary function. Define $g(t)=t^{*}$ for every $t \in[a, b]$. Then the Kurzweil-Henstock $\Delta$-integral $\int_{a}^{b} f(t) \Delta t$ exists, if and only if, the Kurzweil-Henstock-Stieltjes integral $\int_{a}^{b} f^{*}(t) \mathrm{d} g(t)$ exists. In this case, both integrals have the same value.

Proof. For an arbitrary tagged partition $P$ of $[a, b]$, say $P=\left\{\left(\tau_{i},\left[s_{i-1}, s_{i}\right]\right), i=1,2, \ldots, m\right\}$, let

$$
S(P)=\sum_{i=1}^{m} f^{*}\left(\tau_{i}\right)\left[g\left(s_{i}\right)-g\left(s_{i-1}\right)\right]=\sum_{i=1}^{m} f\left(\tau_{i}^{*}\right)\left(s_{i}^{*}-s_{i-1}^{*}\right) .
$$

Assume that $\int_{a}^{b} f(t) \Delta t$ exists. Then, given an arbitrary $\varepsilon>0$, there is a $\Delta$-gauge $\delta=\left(\delta_{L}, \delta_{R}\right)$ on $[a, b]_{\mathbb{T}}$ such that

$$
\left|\sum_{i=1}^{m} f\left(\tau_{i}\right)\left(s_{i}-s_{i-1}\right)-\int_{a}^{b} f(t) \Delta t\right|<\varepsilon
$$

for every $\delta$-fine tagged partition $P=\left\{\left(\tau_{i},\left[s_{i-1}, s_{i}\right]\right), i=1,2, \ldots, m\right\}$ of $[a, b]_{\mathbb{T}}$.

Now, we construct a gauge $\tilde{\delta}:[a, b] \rightarrow \mathbb{R}^{+}$in the following way:

$$
\tilde{\delta}(t)= \begin{cases}\min \left(\delta_{L}(t), \sup \left\{d ; t+d \in[a, b]_{\mathbb{T}}, d \leq \delta_{R}(t)\right\}\right), & t \in(a, b) \cap \mathbb{T}, \\ \sup \left\{d ; a+d \in[a, b]_{\mathbb{T}}, d \leq \delta_{R}(a)\right\}, & t=a, \\ \delta_{L}(b), & t=b, \\ \frac{1}{2} \inf \{|t-s|, s \in \mathbb{T}\}, & t \in[a, b] \backslash \mathbb{T} .\end{cases}
$$

Let $P=\left\{\left(\tau_{i},\left[s_{i-1}, s_{i}\right]\right), i=1,2, \ldots, m\right\}$ be an arbitrary $\tilde{\delta}$-fine tagged partition of $[a, b]$. Then, for every $i \in\{1, \ldots, m\}$, there are two possibilities: either $\tau_{i} \in \mathbb{T}$, or $\left[s_{i-1}, s_{i}\right] \cap \mathbb{T}=\emptyset$. 
The end-points $s_{0}, \ldots, s_{m}$ of subintervals $\left[s_{i-1}, s_{i}\right], i=1,2, \ldots, m$, and tags $\tau_{1}, \ldots, \tau_{m}$ need not belong to $\mathbb{T}$, but we can find a partition $P^{\prime}$ whose end-points of the subintervals and tags belong to $\mathbb{T}$ with $S(P)=S\left(P^{\prime}\right)$, and $P^{\prime}$ is $\delta$-fine. Indeed. We can prove this fact by induction. Clearly, $s_{0}=a \in \mathbb{T}$. Now, consider an interval $\left[s_{i-1}, s_{i}\right]$ with $s_{i-1} \in \mathbb{T}$. Since $\left[s_{i-1}, s_{i}\right] \cap \mathbb{T} \neq \emptyset$, we must have $\tau_{i} \in \mathbb{T}$. If $s_{i} \notin \mathbb{T}$, we replace the partition point $s_{i}$ by $s_{i}^{*}$, delete all partition points $s_{j}$ belonging to $\left(s_{i}, s_{i}^{*}\right)$, and also all tags $\tau_{j}$ belonging to $\left(s_{i}, s_{i}^{*}\right)$. This operation keeps the value of the integral sum (4.5.1) unchanged: the contributions of the intervals $\left[s_{i-1}, s_{i}\right]$ and $\left[s_{i-1}, s_{i}^{*}\right]$ to the value of the sum are the same, and the contributions of intervals $\left[s_{j-1}, s_{j}\right]$ contained in $\left(s_{i}, s_{i}^{*}\right)$ are zero because $s_{j-1}^{*}=s_{j}^{*}=s_{i}^{*}$. It remains to check that the modified partition is $\delta$-fine.

Let $M=\sup \left(\left[a, \tau_{i}+\delta_{R}\left(\tau_{i}\right)\right] \cap \mathbb{T}\right)$. Obviously, $M \in[a, b]_{\mathbb{T}}$. Since our original partition was $\tilde{\delta}$-fine, it follows that

$$
s_{i} \leq \tau_{i}+\tilde{\delta}\left(\tau_{i}\right) \leq \tau_{i}+\sup \left\{d ; \tau_{i}+d \in[a, b]_{\mathbb{T}}, d \leq \delta_{R}\left(\tau_{i}\right)\right\}=M
$$

But $s_{i} \notin \mathbb{T}$ and $M \in \mathbb{T}$ implies $s_{i}^{*} \leq M$, because $s_{i}^{*}$ is the smallest time scale point larger than $s_{i}$. Consequently, $s_{i}^{*} \leq M \leq \tau_{i}+\delta_{R}\left(\tau_{i}\right)$.

Now, $P^{\prime}$ is a $\delta$-fine tagged partition of $[a, b]_{\mathbb{T}}$, and therefore

$$
\left|S(P)-\int_{a}^{b} f(t) \Delta t\right|=\left|S\left(P^{\prime}\right)-\int_{a}^{b} f(t) \Delta t\right|<\varepsilon,
$$

which proves that $\int_{a}^{b} f^{*}(t) \mathrm{d} g(t)$ exists and equals $\int_{a}^{b} f(t) \Delta t$.

Conversely, assume that $\int_{a}^{b} f^{*}(t) \mathrm{d} g(t)$ exists. Then, given an arbitrary $\varepsilon>0$, there is a gauge $\tilde{\delta}:[a, b] \rightarrow \mathbb{R}^{+}$such that

$$
\left|\sum_{i=1}^{m} f\left(\tau_{i}^{*}\right)\left(s_{i}^{*}-s_{i-1}^{*}\right)-\int_{a}^{b} f^{*}(t) \mathrm{d} g(t)\right|<\varepsilon,
$$

for every $\tilde{\delta}$-fine tagged partition of $[a, b]$. We construct a $\Delta$-gauge $\delta=\left(\delta_{L}, \delta_{R}\right)$ on $[a, b]_{\mathbb{T}}$ by letting $\delta_{L}(t)=\tilde{\delta}(t)$ and $\delta_{R}(t)=\max (\tilde{\delta}(t), \mu(t))$ for every $t \in[a, b]_{\mathbb{T}}$.

Consider an arbitrary $\delta$-fine tagged partition $P$ of $[a, b]_{\mathbb{T}}$, say $P=\left\{\left(\tau_{i},\left[s_{i-1}, s_{i}\right]\right), i=\right.$ $1,2, \ldots, m\}$. As we proved above, we can assume, without loss of generality, that the end-points of the subintervals $\left[s_{i-1}, s_{i}\right], i=1,2, \ldots, m$, and the tags $\tau_{i}, i=1,2, \ldots, m$ belong to the time scale $\mathbb{T}$.

Our $\delta$-fine partition need not be $\tilde{\delta}$-fine: for certain values of $i \in\{1, \ldots, m\}$, it can 
happen that $\delta_{R}\left(\tau_{i}\right)+\tau_{i} \geq s_{i}>\tilde{\delta}\left(\tau_{i}\right)+\tau_{i}$. In this case, we have $\delta_{R}\left(\tau_{i}\right)=\mu\left(\tau_{i}\right)$, the point $\tau_{i}$ is right-scattered, and $s_{i}=\sigma\left(\tau_{i}\right)$. We claim that it is possible to find a modified tagged partition $P^{\prime}$ of $[a, b]$ which is $\tilde{\delta}$-fine and $S(P)=S\left(P^{\prime}\right)$. To this end, replace the partition point $s_{i}$ by $\tau_{i}+\tilde{\delta}\left(\tau_{i}\right)$ while keeping $\tau_{i}$ as the tag for the interval $\left[s_{i-1}, \tau_{i}+\tilde{\delta}\left(\tau_{i}\right)\right]$, and cover the interval $\left[\tau_{i}+\tilde{\delta}\left(\tau_{i}\right), s_{i}\right]$ by an arbitrary $\tilde{\delta}$-fine partition. The equality $S(P)=S\left(P^{\prime}\right)$ follows from the fact that $t^{*}=s_{i}$ for every $t \in\left(\tau_{i}, s_{i}\right]$.

The proof is concluded by observing that

$$
\begin{gathered}
\left|\sum_{i=1}^{m} f\left(\tau_{i}\right)\left(s_{i}-s_{i-1}\right)-\int_{a}^{b} f^{*}(t) \mathrm{d} g(t)\right|=\left|S(P)-\int_{a}^{b} f^{*}(t) \mathrm{d} g(t)\right| \\
=\left|S\left(P^{\prime}\right)-\int_{a}^{b} f^{*}(t) \mathrm{d} g(t)\right|<\varepsilon,
\end{gathered}
$$

which implies that $\int_{a}^{b} f(t) \Delta t$ exists and equals $\int_{a}^{b} f^{*}(t) \mathrm{d} g(t)$.

Remark 4.20. Several authors have been interested in Stieltjes-type integrals on time scales (see, for instance, [66] and [87]). For example, the definition of the RiemannStieltjes $\Delta$-integral $\int_{a}^{b} f(t) \Delta g(t)$ of a function $f:[a, b]_{\mathbb{T}} \rightarrow \mathbb{R}^{n}$ with respect to a function $g:[a, b]_{\mathbb{T}} \rightarrow \mathbb{R}$ can be obtained in a straightforward way by taking the definition of the Riemann $\Delta$-integral and replacing the usual integral sums by

$$
\sum_{i=1}^{m} f\left(\tau_{i}\right)\left[g\left(s_{i}\right)-g\left(s_{i-1}\right)\right] .
$$

Alternatively, we can start with the definition of the Kurzweil-Henstock $\Delta$-integral and modify the integral sums in the same way. Using exactly the same reasoning as in the proof of Theorem 4.19, one can show that the resulting Stieltjes-type $\Delta$-integral satisfies

$$
\int_{a}^{b} f(t) \Delta g(t)=\int_{a}^{b} f^{*}(t) \mathrm{d} g^{*}(t) .
$$

Consequently, many properties of the $\Delta$-integrals can be simply derived from the known properties of the Kurzweil-Henstock-Stieltjes integrals.

The next result will be essential to our purposes. Such result is new and can be found in [37], Lemma 4.4.

Lemma 4.21. Let $a, b \in \mathbb{T}, a<b$ and $g(t)=t^{*}$ for every $t \in[a, b]$. If $f:[a, b] \rightarrow \mathbb{R}^{n}$ is 
such that the integral $\int_{a}^{b} f(t) \mathrm{d} g(t)$ exists, then

$$
\int_{c}^{d} f(t) \mathrm{d} g(t)=\int_{c^{*}}^{d^{*}} f(t) \mathrm{d} g(t)
$$

for every $c, d \in[a, b]$.

Proof. Using the definition of the Kurzweil-Henstock-Stieltjes integral and the fact that $g$ is constant on $\left[c, c^{*}\right]$ and on $\left[d, d^{*}\right]$, we obtain $\int_{c}^{c^{*}} f(t) \mathrm{d} g(t)=0$ and $\int_{d}^{d^{*}} f(t) \mathrm{d} g(t)=0$. Therefore

$$
\int_{c}^{d} f(t) \mathrm{d} g(t)=\int_{c}^{c^{*}} f(t) \mathrm{d} g(t)+\int_{c^{*}}^{d} f(t) \mathrm{d} g(t)+\int_{d}^{d^{*}} f(t) \mathrm{d} g(t)=\int_{c^{*}}^{d^{*}} f(t) \mathrm{d} g(t) .
$$

The next result generalizes Theorem 4.11. Notice that we suppressed the hypothesis on the right-dense continuity of the function $f$. The only requirement on $f$ is the $\Delta$ integrability in the sense of Kurzweil-Henstock. It is a new result and can be found in [37].

Theorem 4.22. Let $f: \mathbb{T} \rightarrow \mathbb{R}^{n}$ be a function such that the Kurzweil-Henstock $\Delta$-integral $\int_{a}^{b} f(s) \Delta s$ exists for every $a, b \in \mathbb{T}, a<b$. Choose an arbitrary $a \in \mathbb{T}$ and define

$$
\begin{gathered}
F_{1}(t)=\int_{a}^{t} f(s) \Delta s, \quad t \in \mathbb{T}, \\
F_{2}(t)=\int_{a}^{t} f^{*}(s) \mathrm{d} g(s), \quad t \in \mathbb{T}^{*},
\end{gathered}
$$

where $g(s)=s^{*}$ for every $s \in \mathbb{T}^{*}$. Then $F_{2}=F_{1}^{*}$.

Proof. The statement is a simple consequence of Lemma 4.21 and Theorem 4.19:

$$
F_{2}(t)=\int_{a}^{t} f^{*}(s) \mathrm{d} g(s)=\int_{a}^{t^{*}} f^{*}(s) \mathrm{d} g(s)=\int_{a}^{t^{*}} f(s) \Delta s=F_{1}\left(t^{*}\right)=F_{1}^{*}(t)
$$

Several authors have already considered impulsive dynamic equations on time scales (see, for example, [12], [13], [20], [50]). To this end, let $t_{1}, \ldots, t_{m} \in \mathbb{T}, t_{0} \leq t_{1}<t_{2}<$ $\cdots<t_{m}<t_{0}+\sigma$, where $\sigma>0$, and $I_{1}, \ldots, I_{m}: \mathbb{R}^{n} \rightarrow \mathbb{R}^{n}$. The usual condition which can be found in the existing literature is that the solution should satisfy

$$
x\left(t_{k}^{+}\right)-x\left(t_{k}^{-}\right)=I_{k}\left(x\left(t_{k}^{-}\right)\right), \quad k \in\{1, \ldots, m\} .
$$


The convention is that $x\left(t^{+}\right)=x(t)$ when $t \in \mathbb{T}$ is a right-scattered point and $x\left(t^{-}\right)=x(t)$ when $t \in \mathbb{T}$ is left-scattered. Moreover, it is usually assumed that the solution $x$ should be left-continuous. In this case, equation (4.5.2) reduces to

$$
x\left(t_{k}^{+}\right)-x\left(t_{k}\right)=I_{k}\left(x\left(t_{k}\right)\right), \quad k \in\{1, \ldots, m\} .
$$

Note that if $t_{k}$ is right-scattered, then the left-hand side of equation (4.5.3) is zero. In other words, it makes sense to consider impulses at right-dense points only (the same assumption is made in [13] and [20]).

This motivates us to consider impulsive functional dynamic equations on time scales of the form

$$
\begin{aligned}
x^{\Delta}(t) & =f\left(x_{t}^{*}, t\right), \quad t \in\left[t_{0}, t_{0}+\sigma\right]_{\mathbb{T}} \backslash\left\{t_{1}, \ldots, t_{m}\right\}, \\
\Delta^{+} x\left(t_{k}\right) & =I_{k}\left(x\left(t_{k}\right)\right), \quad k \in\{1, \ldots, m\}, \\
x(t) & =\phi(t), \quad t \in\left[t_{0}-r, t_{0}\right]_{\mathbb{T}},
\end{aligned}
$$

where $r, \sigma>0, t_{1}, \ldots, t_{m} \in \mathbb{T}$ are right-dense points, $t_{0} \leq t_{1}<t_{2}<\cdots<t_{m}<t_{0}+\sigma$, and $I_{1}, \ldots, I_{m}: \mathbb{R}^{n} \rightarrow \mathbb{R}^{n}$. The solution is assumed to be left-continuous. It is not difficult to see that the above problem can be rewritten more compactly in the form

$$
\begin{aligned}
& x(t)=x\left(t_{0}\right)+\int_{t_{0}}^{t} f\left(x_{s}^{*}, s\right) \Delta s+\sum_{\substack{k \in\{1, \ldots, m\} \\
t_{k}<t}} I_{k}\left(x\left(t_{k}\right)\right), \quad t \in\left[t_{0}, t_{0}+\sigma\right]_{\mathbb{T}}, \\
& x(t)=\phi(t), \quad t \in\left[t_{0}-r, t_{0}\right]_{\mathbb{T}} .
\end{aligned}
$$

Our immediate goal is to rewrite this equation as an impulsive measure functional differential equation. In what follows, we present the following theorem which describes the relation between impulsive functional dynamic equations and impulsive measure functional differential equations. Such result is new and it can be found in [37].

Theorem 4.23. Let $\left[t_{0}-r, t_{0}+\sigma\right]_{\mathbb{T}}$ be a time scale interval, $t_{0} \in \mathbb{T}, B \subset \mathbb{R}^{n}, f$ : $G([-r, 0], B) \times\left[t_{0}, t_{0}+\sigma\right]_{\mathbb{T}} \rightarrow \mathbb{R}^{n}$ and $\phi \in G\left(\left[t_{0}-r, t_{0}\right]_{\mathbb{T}}, B\right)$. Define $g(s)=s^{*}$ for every $s \in\left[t_{0}, t_{0}+\sigma\right]$. If $x:\left[t_{0}-r, t_{0}+\sigma\right]_{\mathbb{T}} \rightarrow B$ is a solution of the impulsive functional dynamic 
equation

$$
\begin{aligned}
& x(t)=x\left(t_{0}\right)+\int_{t_{0}}^{t} f\left(x_{s}^{*}, s\right) \Delta s+\sum_{\substack{k \in\{1, \ldots, m\} \\
t_{k}<t}} I_{k}\left(x\left(t_{k}\right)\right), \quad t \in\left[t_{0}, t_{0}+\sigma\right]_{\mathbb{T}}, \\
& x(t)=\phi(t), \quad t \in\left[t_{0}-r, t_{0}\right]_{\mathbb{T}},
\end{aligned}
$$

then $x^{*}:\left[t_{0}-r, t_{0}+\sigma\right] \rightarrow B$ is a solution of the impulsive measure equation

$$
\begin{aligned}
y(t) & =y\left(t_{0}\right)+\int_{t_{0}}^{t} f\left(y_{s}, s^{*}\right) \mathrm{d} g(s)+\sum_{\substack{k \in\{1, \ldots, m\}, t_{k}<t}} I_{k}\left(y\left(t_{k}\right)\right), \quad t \in\left[t_{0}, t_{0}+\sigma\right], \\
y_{t_{0}} & =\phi_{t_{0}}^{*} .
\end{aligned}
$$

Conversely, if $y:\left[t_{0}-r, t_{0}+\sigma\right] \rightarrow B$ satisfies (4.5.6) and (4.5.7), then it must have the form $y=x^{*}$, where $x:\left[t_{0}-r, t_{0}+\sigma\right]_{\mathbb{T}} \rightarrow B$ is a solution of (4.5.4) and (4.5.5).

Proof. Assume that $x$ satisfies (4.5.4) and (4.5.5). Clearly, $x_{t_{0}}^{*}=\phi_{t_{0}}^{*}$. By Theorem 4.22, we have

$$
x^{*}(t)=x^{*}\left(t_{0}\right)+\int_{t_{0}}^{t} f\left(x_{s^{*}}^{*}, s^{*}\right) \mathrm{d} g(s)+\sum_{\substack{k \in\{1, \ldots, m\} \\ t_{k}<t^{*}}} I_{k}\left(x\left(t_{k}\right)\right), \quad t \in\left[t_{0}, t_{0}+\sigma\right] .
$$

We have $t_{k} \in \mathbb{T}$ for every $k \in\{1, \ldots, m\}$. It follows that $x\left(t_{k}\right)=x^{*}\left(t_{k}\right)$ and $t_{k}<t^{*}$, if and only if, $t_{k}<t$. Moreover, since $f\left(x_{s^{*}}^{*}, s^{*}\right)=f\left(x_{s}^{*}, s^{*}\right)$ for every $s \in \mathbb{T}$, we can use Theorem 4.12 to conclude that

$$
x^{*}(t)=x^{*}\left(t_{0}\right)+\int_{t_{0}}^{t} f\left(x_{s}^{*}, s^{*}\right) \mathrm{d} g(s)+\sum_{\substack{k \in\{1, \ldots, m\} \\ t_{k}<t}} I_{k}\left(x^{*}\left(t_{k}\right)\right), \quad t \in\left[t_{0}, t_{0}+\sigma\right],
$$

which proves the first part of the theorem.

Conversely, assume that $y$ satisfies (4.5.6) and (4.5.7). If $t \in \mathbb{T}, t_{0}<u<t \leq t_{0}+\sigma$ and $[u, t) \cap \mathbb{T}=\emptyset$, then $g$ is constant on $[u, t]$ and, therefore, $y(u)=y(t)$. It follows that $y=x^{*}$ for some $x:\left[t_{0}-r, t_{0}+\sigma\right]_{\mathbb{T}} \rightarrow B$. By reversing our previous reasoning, we conclude that $x$ satisfies (4.5.4) and (4.5.5).

The next lemma describes how the Carathéodory and Lipschitz-type conditions concerning the Kurzweil-Henstock-Stieltjes integral of function $f^{*}$ with respect to $g$ can be transferred to the Kurzweil-Henstock $\Delta$-integral of the function $f$. 
Lemma 4.24. Let $\left[t_{0}-r, t_{0}+\sigma\right]_{\mathbb{T}}$ be a time scale interval, $t_{0} \in \mathbb{T}, O=G\left(\left[t_{0}-r, t_{0}+\sigma\right], B\right)$ and $P=G([-r, 0], B), f: P \times\left[t_{0}, t_{0}+\sigma\right]_{\mathbb{T}} \rightarrow \mathbb{R}^{n}$ be an arbitrary function. Define $g(t)=t^{*}$ and $f^{*}(y, t)=f\left(y, t^{*}\right)$ for every $y \in P$ and $t \in\left[t_{0}, t_{0}+\sigma\right]$.

1. If the integral $\int_{t_{0}}^{t_{0}+\sigma} f\left(y_{t}, t\right) \Delta t$ exists for every $y \in O$, then the integral

$$
\int_{t_{0}}^{t_{0}+\sigma} f^{*}\left(y_{t}, t\right) \mathrm{d} g(t)
$$

exists for every $y \in O$.

2. Assume there exists a constant $M>0$ such that

$$
\left|\int_{u_{1}}^{u_{2}} f\left(y_{t}, t\right) \Delta t\right| \leq M\left(u_{2}-u_{1}\right)
$$

for every $y \in O$ and $u_{1}, u_{2} \in\left[t_{0}, t_{0}+\sigma\right]_{\mathbb{T}}, u_{1} \leq u_{2}$. Then,

$$
\left|\int_{u_{1}}^{u_{2}} f^{*}\left(y_{t}, t\right) \mathrm{d} g(t)\right| \leq M\left[g\left(u_{2}\right)-g\left(u_{1}\right)\right]
$$

whenever $t_{0} \leq u_{1} \leq u_{2} \leq t_{0}+\sigma$ and $y \in O$.

3. Assume there exists a constant $L>0$ such that

$$
\left|\int_{u_{1}}^{u_{2}}\left[f\left(y_{t}, t\right)-f\left(z_{t}, t\right)\right] \Delta t\right| \leq L \int_{u_{1}}^{u_{2}}\left\|y_{t}-z_{t}\right\|_{\infty} \Delta t
$$

for every $y, z \in O$ and $u_{1}, u_{2} \in\left[t_{0}, t_{0}+\sigma\right]_{\mathbb{T}}, u_{1} \leq u_{2}$. Then,

$$
\left|\int_{u_{1}}^{u_{2}}\left[f^{*}\left(y_{t}, t\right)-f^{*}\left(z_{t}, t\right)\right] \mathrm{d} g(t)\right| \leq L \int_{u_{1}}^{u_{2}}\left\|y_{t}-z_{t}\right\|_{\infty} \mathrm{d} g(t),
$$

whenever $t_{0} \leq u_{1} \leq u_{2} \leq t_{0}+\sigma$ and $y, z \in O$.

Proof. Consider an arbitrary $y \in O$. If the integral $\int_{t_{0}}^{t_{0}+\sigma} f\left(y_{t}, t\right) \Delta t$ exists, then, using Theorems 4.19 and 4.12 , we have

$$
\int_{t_{0}}^{t_{0}+\sigma} f\left(y_{t}, t\right) \Delta t=\int_{t_{0}}^{t_{0}+\sigma} f\left(y_{t^{*}}, t^{*}\right) \mathrm{d} g(t)=\int_{t_{0}}^{t_{0}+\sigma} f\left(y_{t}, t^{*}\right) \mathrm{d} g(t)=\int_{t_{0}}^{t_{0}+\sigma} f^{*}\left(y_{t}, t\right) \mathrm{d} g(t),
$$

that is, the last integral exists as well. This proves the first part of the theorem.

The remaining two statements follow from Theorem 4.12, Lemma 4.21 and Theorem 
4.19. For the second statement, we have

$$
\begin{gathered}
\left|\int_{u_{1}}^{u_{2}} f^{*}\left(y_{t}, t\right) \mathrm{d} g(t)\right|=\left|\int_{u_{1}}^{u_{2}} f\left(y_{t^{*}}, t^{*}\right) \mathrm{d} g(t)\right|=\left|\int_{u_{1}^{*}}^{u_{2}^{*}} f\left(y_{t^{*}}, t^{*}\right) \mathrm{d} g(t)\right|=\left|\int_{u_{1}^{*}}^{u_{2}^{*}} f\left(y_{t}, t\right) \Delta t\right| \\
\leq M\left(u_{2}^{*}-u_{1}^{*}\right)=M\left[g\left(u_{2}\right)-g\left(u_{1}\right)\right] .
\end{gathered}
$$

For the third statement, we obtain

$$
\begin{array}{r}
\left|\int_{u_{1}}^{u_{2}}\left(f^{*}\left(y_{t}, t\right)-f^{*}\left(z_{t}, t\right)\right) \mathrm{d} g(t)\right|=\left|\int_{u_{1}}^{u_{2}}\left(f\left(y_{t^{*}}, t^{*}\right)-f\left(z_{t^{*}}, t^{*}\right)\right) \mathrm{d} g(t)\right| \\
\quad=\left|\int_{u_{1}^{*}}^{u_{2}^{*}}\left(f\left(y_{t^{*}}, t^{*}\right)-f\left(z_{t^{*}}, t^{*}\right)\right) \mathrm{d} g(t)\right|=\left|\int_{u_{1}^{*}}^{u_{2}^{*}}\left(f\left(y_{t}, t\right)-f\left(z_{t}, t\right)\right) \Delta t\right| \\
\leq L \int_{u_{1}^{*}}^{u_{2}^{*}}\left\|y_{t}-z_{t}\right\|_{\infty} \Delta t=L \int_{u_{1}^{*}}^{u_{2}^{*}}\left\|y_{t}-z_{t}\right\|_{\infty} \mathrm{d} g(t)=L \int_{u_{1}}^{u_{2}}\left\|y_{t}-z_{t}\right\|_{\infty} \mathrm{d} g(t) .
\end{array}
$$





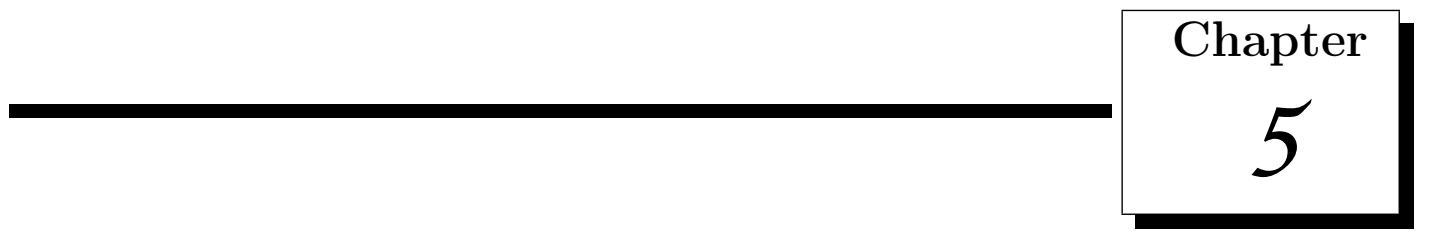

\section{Existence and uniqueness}

In this chapter, we present results concerning local existence and uniqueness of solutions of generalized ODEs, measure FDEs, functional dynamic equations on time scales, impulsive measure FDEs and impulsive functional dynamic equations on time scales.

It worth mentioning that all the results presented in Sections 5.2 and 5.3 of this chapter are new and they follow by using some correspondence between the solutions of the mentioned equations (see Chapter 4) and a known result of existence and uniqueness of solutions of generalized ODEs presented in Section 5.1. These results are contained in [36] and [37].

\subsection{Existence and uniqueness theorem for generalized ODEs}

We start this section by presenting an inequality for generalized ODEs. This inequality will be essential to prove the result following it which concerns existence and uniqueness of solutions for generalized ODEs. It will also be essential to prove a periodic averaging principle for generalized ODEs presented in Chapter 7. Our result is new and it can be found in [84].

Let $X$ be a Banach space with norm $\|\cdot\|$.

Lemma 5.1. Let $O \subset X$ be open and $\Omega=O \times[a, b]$. Assume that $F: \Omega \rightarrow X$ belongs to 
the class $\mathcal{F}(\Omega, h)$. If $x, y:[a, b] \rightarrow O$ are regulated functions, then

$$
\left\|\int_{a}^{b} D[F(x(\tau), t)-F(y(\tau), t)]\right\| \leq \int_{a}^{b}\|x(t)-y(t)\| \mathrm{d} h(t) .
$$

Proof. For an arbitrary tagged partition $\left(\tau_{i},\left[s_{i-1}, s_{i}\right]\right)_{i=1}^{m}$ of $[a, b]$, we have

$$
\begin{gathered}
\left\|\sum_{i=1}^{m}\left[F\left(x\left(\tau_{i}\right), s_{i}\right)-F\left(x\left(\tau_{i}\right), s_{i-1}\right)-F\left(y\left(\tau_{i}\right), s_{i}\right)+F\left(y\left(\tau_{i}\right), s_{i-1}\right)\right]\right\| \leq \\
\sum_{i=1}^{m}\left\|F\left(x\left(\tau_{i}\right), s_{i}\right)-F\left(x\left(\tau_{i}\right), s_{i-1}\right)-F\left(y\left(\tau_{i}\right), s_{i}\right)+F\left(y\left(\tau_{i}\right), s_{i-1}\right)\right\| \leq \\
\sum_{i=1}^{m}\left\|x\left(\tau_{i}\right)-y\left(\tau_{i}\right)\right\|\left[h\left(s_{i}\right)-h\left(s_{i-1}\right)\right],
\end{gathered}
$$

since $F \in \mathcal{F}(\Omega, h)$.

The Kurzweil-Henstock-Stieltjes integral on the right-hand side of inequality (5.1.1) exists, because $h$ is nondecreasing and the function $t \mapsto\|x(t)-y(t)\|$ is regulated. By Lemma 2.14, the integral $\int_{a}^{b} D[F(x(\tau), t)-F(y(\tau), t)]$ also exists. Then given $\varepsilon>0$, there is a gauge $\delta$ of $[a, b]$ such that for every $\delta$-fine tagged partition $\left(\tau_{i},\left[s_{i-1}, s_{i}\right]\right)_{i=1}^{m}$, we have

$$
\begin{gathered}
\| \int_{a}^{b} D[F(x(\tau), t)-F(y(\tau), t)]- \\
\sum_{i=1}^{m}\left[F\left(x\left(\tau_{i}\right), s_{i}\right)-F\left(x\left(\tau_{i}\right), s_{i-1}\right)-F\left(y\left(\tau_{i}\right), s_{i}\right)+F\left(y\left(\tau_{i}\right), s_{i-1}\right)\right] \|<\varepsilon
\end{gathered}
$$

and

$$
\left|\int_{a}^{b}\|x(t)-y(t)\| \mathrm{d} h(t)-\sum_{i=1}^{m}\left\|x\left(\tau_{i}\right)-y\left(\tau_{i}\right)\right\|\left[h\left(s_{i}\right)-h\left(s_{i-1}\right)\right]\right|<\varepsilon .
$$

It follows that

$$
\begin{gathered}
\left\|\int_{a}^{b} D[F(x(\tau), t)-F(y(\tau), t)]\right\| \\
\leq\left\|\sum_{i=1}^{m}\left[F\left(x\left(\tau_{i}\right), s_{i}\right)-F\left(x\left(\tau_{i}\right), s_{i-1}\right)-F\left(y\left(\tau_{i}\right), s_{i}\right)+F\left(y\left(\tau_{i}\right), s_{i-1}\right)\right]\right\| \\
+\| \int_{a}^{b} D[F(x(\tau), t)-F(y(\tau), t)]- \\
\sum_{i=1}^{m}\left[F\left(x\left(\tau_{i}\right), s_{i}\right)-F\left(x\left(\tau_{i}\right), s_{i-1}\right)-F\left(y\left(\tau_{i}\right), s_{i}\right)+F\left(y\left(\tau_{i}\right), s_{i-1}\right)\right] \|
\end{gathered}
$$




$$
\begin{gathered}
<\sum_{i=1}^{m}\left\|x\left(\tau_{i}\right)-y\left(\tau_{i}\right)\right\|\left[h\left(s_{i}\right)-h\left(s_{i-1}\right)\right]+\varepsilon \\
\leq\left|\sum_{i=1}^{m}\left\|x\left(\tau_{i}\right)-y\left(\tau_{i}\right)\right\|\left[h\left(s_{i}\right)-h\left(s_{i-1}\right)\right]-\int_{a}^{b}\|x(t)-y(t)\| \mathrm{d} h(t)\right|+ \\
+\int_{a}^{b}\|x(t)-y(t)\| \mathrm{d} h(t)+\varepsilon<2 \varepsilon+\int_{a}^{b}\|x(t)-y(t)\| \mathrm{d} h(t) .
\end{gathered}
$$

This proves the statement, since $\varepsilon$ can be arbitrarily small.

The next result concerns local existence and uniqueness of a solution for generalized ODEs. Here, we change slightly the proof of this theorem which can be found in [40], Theorem 2.15 .

Theorem 5.2. Let $O \subset X$ open and nondecreasing and $\Omega=O \times[a, b]$. Also, suppose $F: \Omega \rightarrow X$ belongs to the class $\mathcal{F}(\Omega, h)$, where the function $h:[a, b] \rightarrow \mathbb{R}$ is continuous from the left. Then for every $\left(\widetilde{x}, t_{0}\right) \in \Omega$ such that for $\widetilde{x}_{+}=\widetilde{x}+F\left(\widetilde{x}, t_{0}+\right)-F\left(\widetilde{x}, t_{0}\right)$ we have $\left(\widetilde{x}_{+}, t_{0}\right) \in \Omega$ and there exists $a \Delta>0$ such that on the interval $\left[t_{0}, t_{0}+\Delta\right] \subset[a, b]$ there exists a unique solution $x:\left[t_{0}, t_{0}+\Delta\right] \rightarrow X$ of the generalized ordinary differential equation

$$
\frac{d x}{d \tau}=D F(x(\tau), t)
$$

for which $x\left(t_{0}\right)=\widetilde{x}$.

Proof. At first, let $t_{0}$ be a point of continuity of the function $h$, that is, $h\left(t_{0}^{+}\right)=h\left(t_{0}\right)$. Assume that $\Delta>0$ is such that $\left[t_{0}, t_{0}+\Delta\right] \subset(a, b), h\left(t_{0}+\Delta\right)-h\left(t_{0}\right)<\frac{1}{2}$ and the inequality $\|x-\widetilde{x}\| \leq h\left(t_{0}+\Delta\right)-h\left(t_{0}\right)$ implies $x \in O$.

Let $Q$ be the set of functions $z:\left[t_{0}, t_{0}+\Delta\right] \rightarrow X$ such that $z \in G\left(\left[t_{0}, t_{0}+\Delta\right], X\right)$ and $\|z(t)-\widetilde{x}\| \leq h(t)-h\left(t_{0}\right)$ for $t \in\left[t_{0}, t_{0}+\Delta\right]$.

It is not difficult to prove that $Q \subset G\left(\left[t_{0}, t_{0}+\Delta\right], X\right)$ is closed.

For $s \in\left[t_{0}, t_{0}+\Delta\right]$ and $z \in Q$, define

$$
T z(s)=\widetilde{x}+\int_{t_{0}}^{s} D F(z(\tau), t)
$$

The integral on the right-hand side exists and for $s \in\left[t_{0}, t_{0}+\Delta\right]$, we have, by Lemma 2.14 ,

$$
\|T z(s)-\widetilde{x}\|=\left\|\int_{t_{0}}^{s} D F(z(\tau), t)\right\| \leq h(s)-h\left(t_{0}\right),
$$


since $F$ belongs to the class $\mathcal{F}(\Omega, h)$. Thus $T$ maps $Q$ into itself.

Take $t_{0} \leq s_{1}<s_{2} \leq t_{0}+\Delta$ and $z_{1}, z_{2} \in Q$. Then, by Lemma 5.1, we obtain

$$
\begin{gathered}
\left\|T z_{2}\left(s_{2}\right)-T z_{1}\left(s_{2}\right)-\left[T z_{2}\left(s_{1}\right)-T z_{1}\left(s_{1}\right)\right]\right\|= \\
=\left\|\int_{s_{1}}^{s_{2}} D\left[F\left(z_{2}(\tau), t\right)-F\left(z_{1}(\tau), t\right)\right]\right\| \leq \\
\leq \int_{s_{1}}^{s_{2}}\left\|z_{2}(\tau)-z_{1}(\tau)\right\| \mathrm{d} h(t) \leq \sup _{\tau \in\left[s_{1}, s_{2}\right]}\left\|z_{2}(\tau)-z_{1}(\tau)\right\|\left[h\left(s_{2}\right)-h\left(s_{1}\right)\right] \leq \\
\leq \sup _{\tau \in\left[t_{0}, t_{0}+\Delta\right]}\left\|z_{2}(\tau)-z_{1}(\tau)\right\|\left[h\left(s_{2}\right)-h\left(s_{1}\right)\right] \leq\left\|z_{2}-z_{1}\right\|_{\infty}\left[h\left(s_{2}\right)-h\left(s_{1}\right)\right] .
\end{gathered}
$$

Hence,

$$
\left\|T z_{2}-T z_{1}\right\|_{\infty} \leq\left\|z_{2}-z_{1}\right\|_{\infty}\left[h\left(t_{0}+\Delta\right)-h\left(t_{0}\right)\right]<\frac{1}{2}\left\|z_{2}-z_{1}\right\|_{\infty}
$$

and $T$ is a contraction. Thus, by the Banach fixed point theorem, the result follows.

Now, we consider that $t_{0}$ is not a point of continuity of $h$. Define a function $\widetilde{h}$ : $\left[t_{0}, t_{0}+\Delta\right] \rightarrow \mathbb{R}$ as follows

$$
\widetilde{h}(t)=\left\{\begin{array}{l}
h(t), \text { for } t=t_{0} \\
h(t)-h\left(t_{0}^{+}\right), \text {for } t>t_{0} .
\end{array}\right.
$$

Then the function $\widetilde{h}$ is continuous at $t_{0}$, continuous from the left on $\left(t_{0}, t_{0}+\Delta\right]$ and nondecreasing. Defining $\widetilde{F}(x, t)=F(x, t)$ for $t=t_{0}$ and $\widetilde{F}(x, t)=F(x, t)-\left[F\left(\widetilde{x}, t_{0}+\right)-\right.$ $\left.F\left(\widetilde{x}, t_{0}\right)\right]$ for $t>t_{0}$, it is easy to check that $\widetilde{F} \in \mathcal{F}(\Omega, \widetilde{h})$ and, as above, a solution $z$ of $\frac{d z}{d \tau}=D \widetilde{F}(z, t)$ with $z\left(t_{0}\right)=\widetilde{x}_{+}$exists. Defining $x\left(t_{0}\right)=\widetilde{x}$ and $x(t)=z(t)$ for $t>t_{0}$, we have a solution of (5.1.2) for which $x\left(t_{0}\right)=\widetilde{x}$.

Remark 5.3. An estimate for the value $\Delta$ which corresponds to the length of the interval where the solution exists can be obtained by inspection of the proof of this theorem. Let the closed ball $\left\{x \in X,\left\|x-x_{0}\right\| \leq r\right\}$ be denoted by $\bar{B}\left(x_{0}, r\right)$. Then, $\Delta \in(0, \sigma]$ can be any number such that $\bar{B}\left(x_{0}, h\left(t_{0}+\Delta\right)-h\left(t_{0}^{+}\right)\right) \subset O$ and $h\left(t_{0}+\Delta\right)-h\left(t_{0}^{+}\right)<1$. Note that in the proof of the theorem, we assume that $h\left(t_{0}+\Delta\right)-h\left(t_{0}^{+}\right)<1 / 2$, but a careful examination reveals that $h\left(t_{0}+\Delta\right)-h\left(t_{0}^{+}\right)<1$ is sufficient. 


\subsection{Measure FDEs and functional dynamic equations on time scales}

In this section, we prove local existence and uniqueness theorems for measure FDEs and for functional dynamic equations on time scales. These results are new and they can be found in [36].

Before we present the first result, we describe some conditions which the function $f: P \times\left[t_{0}, t_{0}+\sigma\right] \rightarrow \mathbb{R}^{n}$ must fulfill. Such conditions will be referred to throughout this chapter.

(A) The integral $\int_{t_{0}}^{t_{0}+\sigma} f\left(y_{s}, s\right) \mathrm{d} g(s)$ exists for every $y \in O$.

(B) There exists a constant $M>0$ such that

$$
\left|\int_{u_{1}}^{u_{2}} f\left(y_{s}, s\right) \mathrm{d} g(s)\right| \leq M\left[g\left(u_{2}\right)-g\left(u_{1}\right)\right]
$$

for every $y \in O$ and every $u_{1}, u_{2} \in\left[t_{0}, t_{0}+\sigma\right]$.

(C) There exists a constant $L>0$ such that

$$
\left|\int_{u_{1}}^{u_{2}}\left[f\left(y_{s}, s\right)-f\left(z_{s}, s\right)\right] \mathrm{d} g(s)\right| \leq L \int_{u_{1}}^{u_{2}}\left\|y_{s}-z_{s}\right\|_{\infty} \mathrm{d} g(s)
$$

for every $y, z \in O$ and every $u_{1}, u_{2} \in\left[t_{0}, t_{0}+\sigma\right]$.

Also, we consider a function $F: O \times\left[t_{0}, t_{0}+\sigma\right] \rightarrow G\left(\left[t_{0}-r, t_{0}+\sigma\right], \mathbb{R}^{n}\right)$ given by

$$
F(x, t)(\vartheta)= \begin{cases}0, & t_{0}-r \leq \vartheta \leq t_{0}, \\ \int_{t_{0}}^{\vartheta} f\left(x_{s}, s\right) \mathrm{d} g(s), & t_{0} \leq \vartheta \leq t \leq t_{0}+\sigma, \\ \int_{t_{0}}^{t} f\left(x_{s}, s\right) \mathrm{d} g(s), & t \leq \vartheta \leq t_{0}+\sigma,\end{cases}
$$

where $O$ is a subset of $G\left(\left[t_{0}-r, t_{0}+\sigma\right], \mathbb{R}^{n}\right)$ which has the prolongation property, that is, for every $y \in O$ and every $\bar{t} \in\left[t_{0}-r, t_{0}+\sigma\right]$, the function $\bar{y}$ given by

$$
\bar{y}(t)= \begin{cases}y(t), & t_{0}-r \leq t \leq \bar{t} \\ y(\bar{t}), & \bar{t}<t \leq t_{0}+\sigma\end{cases}
$$


is also an element of $O$.

Theorem 5.4. Assume that $X$ is a closed subspace of $G\left(\left[t_{0}-r, t_{0}+\sigma\right], \mathbb{R}^{n}\right), O$ is an open subset of $X$ with the prolongation property, $P=\left\{x_{t} ; x \in O, t \in\left[t_{0}, t_{0}+\sigma\right]\right\}, g$ : $\left[t_{0}, t_{0}+\sigma\right] \rightarrow \mathbb{R}$ is a left-continuous and nondecreasing function and $f: P \times\left[t_{0}, t_{0}+\sigma\right] \rightarrow \mathbb{R}^{n}$ satisfies conditions $(\mathrm{A}),(\mathrm{B})$ and $(\mathrm{C})$. Let $F: O \times\left[t_{0}, t_{0}+\sigma\right] \rightarrow G\left(\left[t_{0}-r, t_{0}+\sigma\right], \mathbb{R}^{n}\right)$ be given by (5.2.1) and assume that $F(x, t) \in X$ for every $x \in O$ and $t \in\left[t_{0}, t_{0}+\sigma\right]$. If $\phi \in P$ is such that the function

$$
z(t)= \begin{cases}\phi\left(t-t_{0}\right), & t \in\left[t_{0}-r, t_{0}\right], \\ \phi(0)+f\left(\phi, t_{0}\right) \Delta^{+} g\left(t_{0}\right), & t \in\left(t_{0}, t_{0}+\sigma\right]\end{cases}
$$

belongs to $O$, then there exists $\delta>0$ and a function $y:\left[t_{0}-r, t_{0}+\delta\right] \rightarrow \mathbb{R}^{n}$ which is a unique solution of the problem

$$
\left\{\begin{array}{l}
y(t)=y\left(t_{0}\right)+\int_{t_{0}}^{t} f\left(y_{s}, s\right) \mathrm{d} g(s) \\
y_{t_{0}}=\phi
\end{array}\right.
$$

Proof. According to Lemma 4.2, the function $F$ belongs to the class $\mathcal{F}\left(O \times\left[t_{0}, t_{0}+\sigma\right], h\right)$, where

$$
h(t)=(M+L)\left[g(t)-g\left(t_{0}\right)\right], \quad t \in\left[t_{0}, t_{0}+\sigma\right] .
$$

Let

$$
x_{0}(\vartheta)= \begin{cases}\phi\left(\vartheta-t_{0}\right), & \vartheta \in\left[t_{0}-r, t_{0}\right], \\ \phi(0), & \vartheta \in\left[t_{0}, t_{0}+\sigma\right] .\end{cases}
$$

It is clear that $x_{0} \in O$. We also claim that $x_{0}+F\left(x_{0}, t_{0}^{+}\right)-F\left(x_{0}, t_{0}\right) \in O$. At first, note that $F\left(x_{0}, t_{0}\right)=0$. The limit $F\left(x_{0}, t_{0}^{+}\right)$is taken with respect to the supremum norm and we know it must exist since $F$ is regulated with respect to the second variable (this follows from the fact that $\left.F \in \mathcal{F}\left(O \times\left[t_{0}, t_{0}+\sigma\right], h\right)\right)$. Thus, it is sufficient to evaluate the pointwise limit $F\left(x_{0}, t_{0}^{+}\right)(\vartheta)$ for every $\vartheta \in\left[t_{0}-r, t_{0}+\sigma\right]$. Using Corollary 2.7, we obtain

$$
F\left(x_{0}, t_{0}^{+}\right)(\vartheta)= \begin{cases}0, & \vartheta \in\left[t_{0}-r, t_{0}\right], \\ f\left(\phi, t_{0}\right) \Delta^{+} g\left(t_{0}\right), & \vartheta \in\left(t_{0}, t_{0}+\sigma\right] .\end{cases}
$$

Hence $x_{0}+F\left(x_{0}, t_{0}^{+}\right)-F\left(x_{0}, t_{0}\right)=z \in O$.

Since all the assumptions of Theorem 5.2 are satisfied, there exists $\delta>0$ and a unique 
solution $x:\left[t_{0}, t_{0}+\delta\right] \rightarrow X$ of the generalized ordinary differential equation

$$
\frac{\mathrm{d} x}{\mathrm{~d} \tau}=D F(x, t), \quad x\left(t_{0}\right)=x_{0}
$$

According to Theorem 4.6, the function $y:\left[t_{0}-r, t_{0}+\delta\right] \rightarrow O$ given by

$$
y(\vartheta)= \begin{cases}x\left(t_{0}\right)(\vartheta), & t_{0}-r \leq \vartheta \leq t_{0} \\ x(\vartheta)(\vartheta), & t_{0} \leq \vartheta \leq t_{0}+\delta\end{cases}
$$

is a solution of the problem

$$
\left\{\begin{array}{l}
y(t)=y\left(t_{0}\right)+\int_{t_{0}}^{t} f\left(y_{s}, s\right) \mathrm{d} g(s) \\
y_{t_{0}}=\phi
\end{array}\right.
$$

This solution must be unique. Otherwise, Theorem 4.5 would imply that $x$ is not the only solution of the generalized ordinary differential equation (5.2.3).

Remark 5.5. Since the assumptions of Theorem 5.4 might look complicated, it worths mentioning two typical choices for the sets $X, O$ and $P$ :

- $g(t)=t$ for every $t \in\left[t_{0}, t_{0}+\sigma\right], X=C\left(\left[t_{0}-r, t_{0}+\sigma\right], \mathbb{R}^{n}\right), B \subset \mathbb{R}^{n}$ is an open set, $O=C\left(\left[t_{0}-r, t_{0}+\sigma\right], B\right), P=C([-r, 0], B)$. Both conditions $F(x, t) \in X$ and $z \in O$ from Theorem 5.4 are always satisfied (by Corollary 2.7, $F(x, t)$ is a continuous function and therefore $F(x, t) \in X)$.

- $X=G\left(\left[t_{0}-r, t_{0}+\sigma\right], \mathbb{R}^{n}\right), B \subset \mathbb{R}^{n}$ is an open set, $O=G\left(\left[t_{0}-r, t_{0}+\sigma\right], B\right)$, $P=G([-r, 0], B)$. The condition $F(x, t) \in X$ from Theorem 5.4 is always satisfied (by Corollary 2.7, $F(x, t)$ is a regulated function and therefore $F(x, t) \in X$ ). The condition $z \in O$ reduces to $\phi(0)+f\left(\phi, t_{0}\right) \Delta^{+} g\left(t_{0}\right) \in B$. Note that if

$$
y(t)=y\left(t_{0}\right)+\int_{t_{0}}^{t} f\left(y_{s}, s\right) \mathrm{d} g(s),
$$

then $y\left(t_{0}^{+}\right)=\phi(0)+f\left(\phi, t_{0}\right) \Delta^{+} g\left(t_{0}\right)$. In other words, the solution does not leave the set $B$ immediately after time $t_{0}$.

In both cases, we can use Remark 5.3 to obtain an estimate for the value $\delta$ which corresponds to the length of interval where the solution exists. Assume there exists 
$\rho>0$ such that $|y-\phi(t)|<\rho$ implies $y \in B$ for every $t \in[-r, 0]$ (in other words, a $\rho$-neighborhood of $\phi$ is contained in $B$ ). Since we have

$$
h(t)=(M+L)\left[g(t)-g\left(t_{0}\right)\right], \quad t \in\left[t_{0}, t_{0}+\sigma\right],
$$

then $\delta \in(0, \sigma]$ can be any number such that

$$
g\left(t_{0}+\delta\right)-g\left(t_{0}^{+}\right)<\frac{\min (1, \rho)}{M+L}
$$

obtaining an estimate for the value of $\delta$.

In what follows, we prove a local existence and uniqueness theorem for functional dynamic equations on time scales. This is also an original result.

Theorem 5.6. Let $\left[t_{0}-r, t_{0}+\sigma\right]_{\mathbb{T}}$ be a time scale interval, $t_{0} \in \mathbb{T}, B \subset \mathbb{R}^{n}$ be open, $C=C\left(\left[t_{0}-r, t_{0}+\sigma\right]_{\mathbb{T}}, B\right), P=\left\{y_{t}^{*} ; y \in C, t \in\left[t_{0}, t_{0}+\sigma\right]\right\}, f: P \times\left[t_{0}, t_{0}+\sigma\right]_{\mathbb{T}} \rightarrow \mathbb{R}^{n}$ satisfies the following conditions:

$\left(\mathrm{A}^{*}\right)$ For every $y \in C$, the function $t \mapsto f\left(y_{t}^{*}, t\right)$ is rd-continuous on $\left[t_{0}, t_{0}+\sigma\right]_{\mathbb{T}}$.

(B*) There exists a constant $M>0$ such that

$$
\left|\int_{u_{1}}^{u_{2}} f\left(y_{s}, s\right) \Delta s\right| \leq M\left(u_{2}-u_{1}\right)
$$

for every $y \in O$ and every $u_{1}, u_{2} \in\left[t_{0}, t_{0}+\sigma\right]_{\mathbb{T}}$.

$\left(\mathrm{C}^{*}\right)$ There exists a constant $L>0$ such that

$$
\left|\int_{u_{1}}^{u_{2}}\left[f\left(y_{s}, s\right)-f\left(z_{s}, s\right)\right] \Delta s\right| \leq L \int_{u_{1}}^{u_{2}}\left\|y_{s}-z_{s}\right\|_{\infty} \Delta s
$$

for every $y, z \in O$ and every $u_{1}, u_{2} \in\left[t_{0}, t_{0}+\sigma\right]_{\mathbb{T}}$.

If $\phi:\left[t_{0}-r, t_{0}\right]_{\mathbb{T}} \rightarrow B$ is a continuous function such that $\phi\left(t_{0}\right)+f\left(\phi_{t_{0}}^{*}, t_{0}\right) \mu\left(t_{0}\right) \in B$, then there exist $\delta>0$ such that $\delta \geq \mu\left(t_{0}\right)$ and $t_{0}+\delta \in \mathbb{T}$, and a function $y:\left[t_{0}-r, t_{0}+\delta\right]_{\mathbb{T}} \rightarrow B$ which is the unique solution of the functional dynamic equation

$$
\left\{\begin{array}{l}
y^{\Delta}(t)=f\left(y_{t}^{*}, t\right), \quad t \in\left[t_{0}, t_{0}+\delta\right] \\
y(t)=\phi(t), \quad t \in\left[t_{0}-r, t_{0}\right]_{\mathbb{T}} .
\end{array}\right.
$$


Proof. Let $X=\left\{y^{*} ; y \in C\left(\left[t_{0}-r, t_{0}+\sigma\right]_{\mathbb{T}}, \mathbb{R}^{n}\right)\right\}, O=\left\{y^{*} ; y \in C\right\}$, and $g(t)=t^{*}$ for every $t \in\left[t_{0}, t_{0}+\sigma\right]$. Note that $C\left(\left[t_{0}-r, t_{0}+\sigma\right]_{\mathbb{T}}, \mathbb{R}^{n}\right)$ is a closed (Banach) space and the operator $T: C\left(\left[t_{0}-r, t_{0}+\sigma\right]_{\mathbb{T}}, \mathbb{R}^{n}\right) \rightarrow X$ given by $T(y)=y^{*}$ is an isometric isomorphism. It follows that $X$ is a closed subspace of $G\left(\left[t_{0}-r, t_{0}+\sigma\right], \mathbb{R}^{n}\right)$.

The function $F: O \times\left[t_{0}, t_{0}+\sigma\right] \rightarrow G\left(\left[t_{0}-r, t_{0}+\sigma\right], \mathbb{R}^{n}\right)$ given by (5.2.1) satisfies $F(x, t) \in X$ for every $x \in O, t \in\left[t_{0}, t_{0}+\sigma\right]$. Indeed, by (5.2.1), it follows that $F(x, t)$ is constant in $\left[t_{0}-r, t_{0}\right]$ and in every interval $(\alpha, \beta) \subset\left[t_{0}, t_{0}+r\right]$ which contains no time scale points (because $g$ is constant in such intervals).

The function $g$ is left-continuous, and it follows from Corollary 2.7 that $F(x, t)$ is left-continuous on $\left[t_{0}, t_{0}+\sigma\right]$ and right-continuous at all points of $\left[t_{0}, t_{0}+\sigma\right]$ where $g$ is right-continuous, that is, at all right-dense points of $\left[t_{0}, t_{0}+r\right]_{\mathbb{T}}$. Thus $F(x, t)$ must have the form $y^{*}$ for some rd-continuous function $y:\left[t_{0}-r, t_{0}+\sigma\right]_{\mathbb{T}} \rightarrow \mathbb{R}^{n}$ and $F(x, t) \in X$.

It is also clear that $O$ is an open subset of $X$ and has the prolongation property.

Let $f^{*}(y, t)=f\left(y, t^{*}\right)$ for every $y \in P$ and $t \in\left[t_{0}, t_{0}+\sigma\right]$. Consider an arbitrary $y \in O$. Since $t \mapsto f\left(y_{t}, t\right)$ is rd-continuous on $\left[t_{0}, t_{0}+\sigma\right]_{\mathbb{T}}$, the integral $\int_{t_{0}}^{t_{0}+\sigma} f\left(y_{t}, t\right) \Delta t$ exists. Using Theorem 4.11 and Theorem 4.12, we have

$$
\int_{t_{0}}^{t_{0}+\sigma} f\left(y_{t}, t\right) \Delta t=\int_{t_{0}}^{t_{0}+\sigma} f\left(y_{t^{*}}, t^{*}\right) \mathrm{d} g(t)=\int_{t_{0}}^{t_{0}+\sigma} f\left(y_{t}, t^{*}\right) \mathrm{d} g(t)=\int_{t_{0}}^{t_{0}+\sigma} f^{*}\left(y_{t}, t\right) \mathrm{d} g(t),
$$

that is, the last integral exists.

Since $\Delta^{+} g\left(t_{0}\right)=\mu\left(t_{0}\right)$ and $\phi\left(t_{0}\right)+f\left(\phi_{t_{0}}^{*}, t_{0}\right) \mu\left(t_{0}\right) \in B$, it follows that the function

$$
z(t)= \begin{cases}\phi_{t_{0}}^{*}\left(t-t_{0}\right), & t \in\left[t_{0}-r, t_{0}\right] \\ \phi_{t_{0}}^{*}(0)+f\left(\phi_{t_{0}}^{*}, t_{0}\right) \Delta^{+} g\left(t_{0}\right), & t \in\left(t_{0}, t_{0}+\sigma\right]\end{cases}
$$

belongs to $O$. Therefore the functions $f^{*}, g(s)=s^{*}$ and $\phi_{t_{0}}^{*}$ satisfy all assumptions of Theorem 5.4, and there exist $\delta>0$ and a function $u:\left[t_{0}-r, t_{0}+\delta\right] \rightarrow B$ which is the unique solution of

$$
\left\{\begin{array}{l}
u(t)=u\left(t_{0}\right)+\int_{t_{0}}^{t} f^{*}\left(u_{s}, s\right) \mathrm{d} g(s), t \in\left[t_{0}, t_{0}+\delta\right] \\
u_{t_{0}}=\phi_{t_{0}}^{*}
\end{array}\right.
$$


By Theorem 4.13, $u=y^{*}$, where $y:\left[t_{0}-r, t_{0}+\delta\right]_{\mathbb{T}} \rightarrow B$ is a solution of

$$
\left\{\begin{array}{l}
y^{\Delta}(t)=f\left(y_{t}^{*}, t\right), \quad t \in\left[t_{0}, t_{0}+\delta\right] \\
y(t)=\phi(t), \quad t \in\left[t_{0}-r, t_{0}\right]_{\mathbb{T}} .
\end{array}\right.
$$

Without loss of generality, we can assume that $\delta \geq \mu\left(t_{0}\right)$. Otherwise, let $y\left(\sigma\left(t_{0}\right)\right)=$ $\phi\left(t_{0}\right)+f\left(\phi_{t_{0}}^{*}, t_{0}\right) \mu\left(t_{0}\right)$ to obtain a solution defined on $\left[t_{0}-r, t_{0}+\mu\left(t_{0}\right)\right]_{\mathbb{T}}$. Again by Theorem 4.13, it follows that the solution $y$ is unique.

Remark 5.7. Similarly to the previous existence-uniqueness results, we can estimate the value of $\delta$ which corresponds to length of the interval where the solution exists. Assume that there exists $\rho>0$ such that $|y-\phi(t)|<\rho$ implies $y \in B$ for every $t \in\left[t_{0}-r, t_{0}\right]_{\mathbb{T}}$ (in other words, a $\rho$-neighborhood of $\phi$ is contained in $B$ ). By Remark 5.5, we know that $\delta \in(0, \sigma]$ can be any number satisfying

$$
g\left(t_{0}+\delta\right)-g\left(t_{0}+\right)<\frac{\min (1, \rho)}{M+L}
$$

In our particular case, we have $g(t)=t^{*}$ for every $t \in\left[t_{0}, t_{0}+\sigma\right]$. Since $g\left(t_{0}+\delta\right)=t_{0}+\delta$ and $g\left(t_{0}+\right)=\sigma\left(t_{0}\right)$, we obtain

$$
\delta<\mu\left(t_{0}\right)+\frac{\min (1, \rho)}{M+L}
$$

which is the estimative for $\delta$.

\subsection{Existence and uniqueness for impulsive equations}

In this section, we present results concerning local existence and uniqueness of a solution for impulsive measure functional differential equation and for functional dynamic equation on time scales involving impulses. They are new results and they can be found in $[36]$.

Theorem 5.8. Assume that $X=G\left(\left[t_{0}-r, t_{0}+\sigma\right], \mathbb{R}^{n}\right), B \subset \mathbb{R}^{n}$ is an open set, $O=$ $G\left(\left[t_{0}-r, t_{0}+\sigma\right], B\right), P=G([-r, 0], B), m \in \mathbb{N}, t_{0} \leq t_{1}<t_{2}<\ldots<t_{m}<t_{0}+\sigma$, $g:\left[t_{0}, t_{0}+\sigma\right] \rightarrow \mathbb{R}$ is a left-continuous nondecreasing function which is continuous at $t_{1}, \ldots, t_{m}$. Also, suppose $I_{1}, \ldots, I_{m}: B \rightarrow \mathbb{R}^{n}$ and $f: P \times\left[t_{0}, t_{0}+\sigma\right] \rightarrow \mathbb{R}^{n}$ satisfy the following conditions:

1. The integral $\int_{t_{0}}^{t_{0}+\sigma} f\left(y_{t}, t\right) \mathrm{d} g(t)$ exists for every $y \in O$. 
2. There exists a constant $M_{1}>0$ such that

$$
\left|\int_{u_{1}}^{u_{2}} f\left(y_{t}, t\right) \mathrm{d} g(t)\right| \leq M_{1}\left[g\left(u_{2}\right)-g\left(u_{1}\right)\right]
$$

whenever $t_{0} \leq u_{1} \leq u_{2} \leq t_{0}+\sigma$ and $y \in O$.

3. There exists a constant $L_{1}>0$ such that

$$
\left|\int_{u_{1}}^{u_{2}}\left[f\left(y_{t}, t\right)-f\left(z_{t}, t\right)\right] \mathrm{d} g(t)\right| \leq L_{1} \int_{u_{1}}^{u_{2}}\left\|y_{t}-z_{t}\right\|_{\infty} \mathrm{d} g(t)
$$

whenever $t_{0} \leq u_{1} \leq u_{2} \leq t_{0}+\sigma$ and $y, z \in O$.

4. There exists a constant $M_{2}>0$ such that

$$
\left|I_{k}(x)\right| \leq M_{2}
$$

for every $k \in\{1, \ldots, m\}$ and $x \in B$.

5. There exists a constant $L_{2}>0$ such that

$$
\left|I_{k}(x)-I_{k}(y)\right| \leq L_{2}|x-y|,
$$

for every $k \in\{1, \ldots, m\}$ and $x, y \in B$.

Let $\phi \in P$ and assume that either $t_{0}<t_{1}$ and $\phi(0)+f\left(\phi, t_{0}\right) \Delta^{+} g\left(t_{0}\right) \in B$, or $t_{0}=t_{1}$ and $\phi(0)+I_{1}(\phi(0)) \in B$. Then there exist $\delta>0$ and a function $y:\left[t_{0}-r, t_{0}+\delta\right] \rightarrow \mathbb{R}^{n}$ which is the unique solution of the impulsive measure functional differential equation

$$
\left\{\begin{array}{l}
y(t)=y\left(t_{0}\right)+\int_{t_{0}}^{t} f\left(y_{s}, s\right) \mathrm{d} g(s)+\sum_{\substack{k \in\{1, \ldots, m\}, t_{k}<t}} I_{k}\left(y\left(t_{k}\right)\right), \quad t \in\left[t_{0}, t_{0}+\delta\right], \\
y_{t_{0}}=\phi .
\end{array}\right.
$$

Proof. For every $y \in P$, define

$$
\tilde{f}(y, t)= \begin{cases}f(y, t), & t \in\left[t_{0}, t_{0}+\sigma\right] \backslash\left\{t_{1}, \ldots, t_{m}\right\} \\ I_{k}(y(0)), & t=t_{k} \text { for some } k \in\{1, \ldots, m\} .\end{cases}
$$

Moreover, let $c_{1}, \ldots, c_{m} \in \mathbb{R}$ be constants such that the function $\tilde{g}:\left[t_{0}, t_{0}+\sigma\right] \rightarrow \mathbb{R}$ given 
by

$$
\tilde{g}(t)= \begin{cases}g(t), & t \in\left[t_{0}, t_{1}\right], \\ g(t)+c_{k}, & t \in\left(t_{k}, t_{k+1}\right] \text { for some } k \in\{1, \ldots, m-1\}, \\ g(t)+c_{m}, & t \in\left(t_{m}, t_{0}+\sigma\right],\end{cases}
$$

satisfies $\Delta^{+} \tilde{g}\left(t_{k}\right)=1$ for every $k \in\{1, \ldots, m\}$.

Since $g$ is a nondecreasing and left-continuous function, $\tilde{g}$ must have the same properties. Then either $t_{0}<t_{1}$ and $\phi(0)+\tilde{f}\left(\phi, t_{0}\right) \Delta^{+} \tilde{g}\left(t_{0}\right)=\phi(0)+f\left(\phi, t_{0}\right) \Delta^{+} g\left(t_{0}\right) \in B$, or $t_{0}=t_{1}$ and $\phi(0)+\tilde{f}\left(\phi, t_{0}\right) \Delta^{+} \tilde{g}\left(t_{0}\right)=\phi(0)+I_{1}(\phi(0)) \in B$.

Using these facts and Lemma 4.18, we see that the functions $\tilde{f}, \tilde{g}$, and $\phi$ satisfy all hypotheses of Theorem 5.4. Consequently, there exist $\delta>0$ and a function $y$ : $\left[t_{0}-r, t_{0}+\delta\right] \rightarrow \mathbb{R}^{n}$ which is the unique solution of the problem

$$
\left\{\begin{array}{l}
y(t)=y\left(t_{0}\right)+\int_{t_{0}}^{t} \tilde{f}\left(y_{s}, s\right) \mathrm{d} \tilde{g}(s) \\
y_{t_{0}}=\phi
\end{array}\right.
$$

Finally, by Theorem 4.16, the function $y$ is also the unique solution of (5.3.1) on $\left[t_{0}-\right.$ $\left.r, t_{0}+\delta\right]$.

In the sequel, we prove a result on local existence and uniqueness of solutions of impulsive functional dynamic equations on time scales. It is a new result.

Theorem 5.9. Assume that $\left[t_{0}-r, t_{0}+\sigma\right]_{\mathbb{T}}$ is a time scale interval, $t_{0} \in \mathbb{T}, B \subset \mathbb{R}^{n}$ is an open set, $O=G\left(\left[t_{0}-r, t_{0}+\sigma\right], B\right), P=G([-r, 0], B), m \in \mathbb{N}, t_{1}, \ldots, t_{m} \in\left[t_{0}, t_{0}+\sigma\right]_{\mathbb{T}}$ are right-dense points such that $t_{0} \leq t_{1}<\cdots<t_{m}<t_{0}+\sigma$. Let $f: P \times\left[t_{0}, t_{0}+\sigma\right]_{\mathbb{T}} \rightarrow \mathbb{R}^{n}$ and $I_{1}, \ldots, I_{m}: B \rightarrow \mathbb{R}^{n}$ be functions which satisfy the following conditions:

1. The integral $\int_{t_{0}}^{t_{0}+\sigma} f\left(y_{t}, t\right) \Delta t$ exists for every $y \in O$.

2. There exists a constant $M_{1}>0$ such that

$$
\left|\int_{u_{1}}^{u_{2}} f\left(y_{t}, t\right) \Delta t\right| \leq M_{1}\left(u_{2}-u_{1}\right)
$$

for every $y \in O$ and $u_{1}, u_{2} \in\left[t_{0}, t_{0}+\sigma\right]_{\mathbb{T}}, u_{1} \leq u_{2}$.

3. There exists a constant $L_{1}>0$ such that

$$
\left|\int_{u_{1}}^{u_{2}}\left[f\left(y_{t}, t\right)-f\left(z_{t}, t\right)\right] \Delta t\right| \leq L_{1} \int_{u_{1}}^{u_{2}}\left\|y_{t}-z_{t}\right\|_{\infty} \Delta t
$$


for every $y, z \in O$ and $u_{1}, u_{2} \in\left[t_{0}, t_{0}+\sigma\right]_{\mathbb{T}}, u_{1} \leq u_{2}$.

4. There exists a constant $M_{2}>0$ such that

$$
\left|I_{k}(y)\right| \leq M_{2}
$$

for every $k \in\{1, \ldots, m\}$ and $y \in B$.

5. There exists a constant $L_{2}>0$ such that

$$
\left|I_{k}(x)-I_{k}(y)\right| \leq L_{2}|x-y|
$$

for every $k \in\{1, \ldots, m\}$ and $x, y \in B$.

Let $\phi:\left[t_{0}-r, t_{0}\right]_{\mathbb{T}} \rightarrow B$ be a regulated function such that either $t_{0}<t_{1}$ and $\phi\left(t_{0}\right)+$ $f\left(\phi_{t_{0}}^{*}, t_{0}\right) \mu(t) \in B$, or $t_{0}=t_{1}$ and $\phi\left(t_{0}\right)+I_{1}\left(\phi\left(t_{0}\right)\right) \in B$. Then there exist $\delta>0$ such that $\delta \geq \mu\left(t_{0}\right)$ and $t_{0}+\delta \in \mathbb{T}$, and a function $y:\left[t_{0}-r, t_{0}+\delta\right]_{\mathbb{T}} \rightarrow B$ which is the unique solution of the impulsive functional dynamic equation

$$
\left\{\begin{array}{l}
y(t)=y\left(t_{0}\right)+\int_{t_{0}}^{t} f\left(y_{s}^{*}, s\right) \Delta s+\sum_{\substack{k \in\{1, \ldots, m\} \\
t_{k}<t}} I_{k}\left(y\left(t_{k}\right)\right), \quad t \in\left[t_{0}, t_{0}+\delta\right]_{\mathbb{T}}, \\
y(t)=\phi(t), \quad t \in\left[t_{0}-r, t_{0}\right]_{\mathbb{T}} .
\end{array}\right.
$$

Proof. Let $g(t)=t^{*}$ and $f^{*}(y, t)=f\left(y, t^{*}\right)$ for every $t \in\left[t_{0}, t_{0}+\sigma\right]$ and $y \in P$. Note that $\Delta^{+} g\left(t_{0}\right)=\mu\left(t_{0}\right)$. Using the hypotheses and Lemma 4.24, we see that the functions $f^{*}$, $g$ and $\phi_{t_{0}}^{*}$ satisfy all assumptions of Theorem 5.8. Consequently, there exist $\delta>0$ and a function $u:\left[t_{0}-r, t_{0}+\delta\right] \rightarrow B$ which is the unique solution of

$$
\left\{\begin{array}{l}
u(t)=u\left(t_{0}\right)+\int_{t_{0}}^{t} f^{*}\left(u_{s}, s\right) \mathrm{d} g(s)+\sum_{\substack{k \in\{1, \ldots, m\}, t_{k}<t}} I_{k}\left(u\left(t_{k}\right)\right), \quad t \in\left[t_{0}, t_{0}+\delta\right], \\
u_{t_{0}}=\phi_{t_{0}}^{*} .
\end{array}\right.
$$

Then, by Theorem 4.23, $u=y^{*}$, where $y:\left[t_{0}-r, t_{0}+\delta\right]_{\mathbb{T}} \rightarrow B$ is a solution of

$$
\left\{\begin{array}{l}
y(t)=y\left(t_{0}\right)+\int_{t_{0}}^{t} f\left(y_{s}^{*}, s\right) \Delta s+\sum_{\substack{k \in\{1, \ldots, m\} \\
t_{k}<t}} I_{k}\left(y\left(t_{k}\right)\right), \quad t \in\left[t_{0}, t_{0}+\delta\right]_{\mathbb{T}}, \\
y(t)=\phi(t), \quad t \in\left[t_{0}-r, t_{0}\right]_{\mathbb{T}} .
\end{array}\right.
$$


Without loss of generality, we can assume that $\delta \geq \mu\left(t_{0}\right)$. Otherwise, $t_{0}$ is rightscattered, $t_{0}<t_{1}$, and we can let

$$
y\left(\sigma\left(t_{0}\right)\right)=\phi\left(t_{0}\right)+f\left(\phi_{t_{0}}^{*}, t_{0}\right) \mu\left(t_{0}\right)
$$

to obtain a solution defined on $\left[t_{0}-r, t_{0}+\mu\left(t_{0}\right)\right]_{\mathbb{T}}$. Again, by Theorem 4.23 , the solution $y$ is unique. 


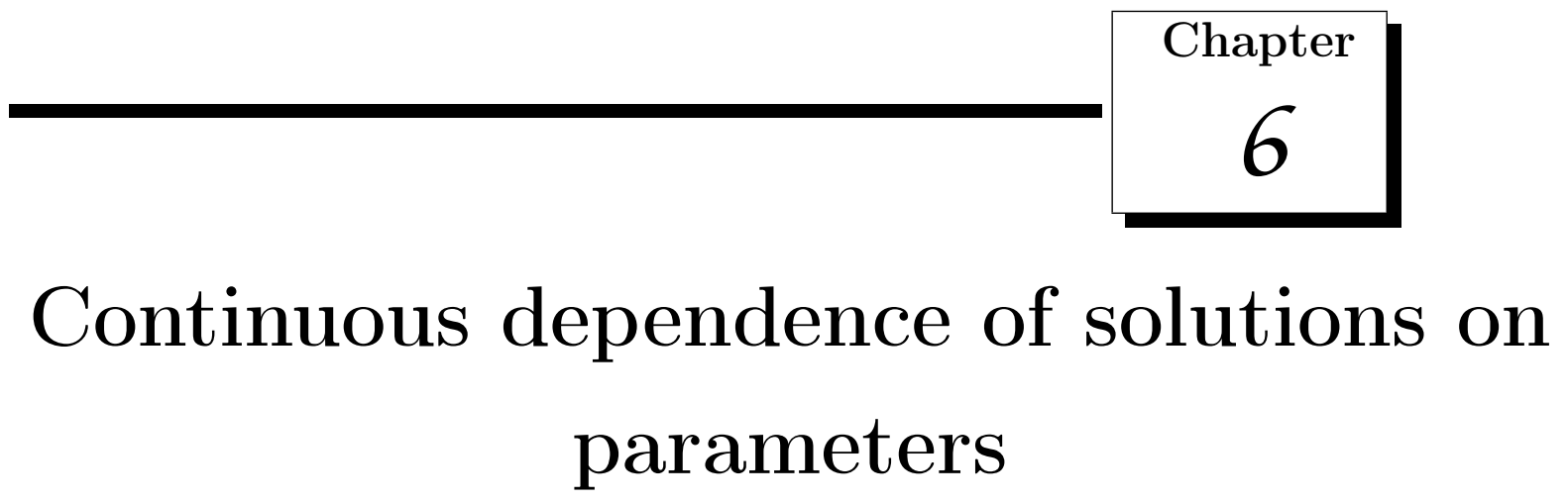

This chapter is divided in two sections. The first one concerns results on continuous dependence of solutions on parameters for generalized ODEs in Banach space, measure functional differential equations, functional dynamic equations on time scales, impulsive measure functional differential equations and impulsive functional dynamic equations on time scales.

In the second section, we investigate whether the solutions of impulsive functional dynamic equations on time scales depend continuously on time scales.

Almost all of results presented in this chapter are new. They are contained in the papers [16], [32], [36], [37] and [45].

\subsection{Continuous dependence on parameters}

Our goal in this section is to prove results on continuous dependence of solutions on parameters for generalized ODEs in Banach spaces, measure functional differential equations, functional dynamic equations on time scales, impulsive measure functional differential equations and also, impulsive functional dynamic equations on time scales.

Let $X$ be a Banach space with norm $\|\cdot\|$. Consider $\Omega=O \times[a, b]$, where $O \subset X$ is open. 
Recall that

$$
\lim _{s \rightarrow t^{+}} F(x, s)=F\left(x, t^{+}\right) \text {and } \lim _{s \rightarrow t^{-}} F(x, s)=F\left(x, t^{-}\right) .
$$

In the sequel, we present a result which is a slightly modified version of Lemma 8.1, from [4].

Lemma 6.1. Consider a sequence of functions $F_{k}: \Omega \rightarrow X$ such that $F_{k} \in \mathcal{F}\left(\Omega, h_{k}\right)$ for every $k=0,1,2, \ldots$ which satisfy

$$
\lim _{k \rightarrow \infty} F_{k}(x, t)=F_{0}(x, t) \text { and } \lim _{k \rightarrow \infty} F_{k}\left(x, t^{+}\right)=F_{0}\left(x, t^{+}\right)
$$

Moreover, assume that $h_{k}:[a, b] \rightarrow \mathbb{R}$ is nondecreasing and left-continuous such that $h_{k}(b)-h_{k}(a) \leq c$, for some $c>0$ and each $k=1,2, \ldots$ Let $\psi_{k} \in G([a, b], X), k=1,2, \ldots$, be such that

$$
\left\|\psi_{k}-\psi_{0}\right\|_{\infty}=\sup _{a \leq t \leq b}\left|\psi_{k}(t)-\psi_{0}(t)\right| \stackrel{k \rightarrow \infty}{\rightarrow} 0
$$

Then, we have

$$
\left\|\int_{a}^{b} D F_{k}\left(\psi_{k}(\tau), s\right)-\int_{c}^{d} D F_{0}\left(\psi_{0}(\tau), s\right)\right\| \stackrel{k \rightarrow \infty}{\rightarrow} 0 .
$$

Proof. Note that $\psi_{0} \in G([a, b], X)$, since $\psi_{0}$ is, by assumption, the uniform limit of regulated functions on $[a, b]$. Then, all the integrals $\int_{a}^{b} D F_{k}\left(\psi_{k}(\tau), s\right)$ exist, for $k=0,1,2, \ldots$ ( See Lemma 2.14).

Assume that $\varepsilon>0$ is given. Since any regulated function can be uniformly approximated by a step function, there is a step function $y:[a, b] \rightarrow X$ such that

$$
\left\|y-\psi_{0}\right\|_{\infty}=\sup _{a \leq t \leq b}\left\|y(t)-\psi_{0}(t)\right\|<\varepsilon
$$

Since $\left\|\psi_{k}-\psi_{0}\right\|_{\infty} \rightarrow 0$ as $k \rightarrow \infty$, there exists a positive integer $N_{0}$ such that

$$
\left\|\psi_{k}-\psi_{0}\right\|_{\infty}<\varepsilon
$$

for all $k>N_{0}$.

Assume that $k>N_{0}$. Then,

$$
\left\|\int_{a}^{b} D F_{k}\left(\psi_{k}(\tau), s\right)-\int_{a}^{b} D F_{0}\left(\psi_{0}(\tau), s\right)\right\| \leq\left\|\int_{a}^{b} D\left[F_{k}\left(\psi_{k}(\tau), s\right)-F_{k}\left(\psi_{0}(\tau), s\right)\right]\right\|+
$$




$$
\begin{gathered}
+\left\|\int_{a}^{b} D\left[F_{k}\left(\psi_{0}(\tau), s\right)-F_{k}(y(\tau), s)\right]\right\|+\left\|\int_{a}^{b} D\left[F_{k}(y(\tau), s)-F_{0}(y(\tau), s)\right]\right\|+ \\
+\left\|\int_{a}^{b} D\left[F_{0}(y(\tau), s)-F_{0}\left(\psi_{0}(\tau), s\right)\right]\right\| .
\end{gathered}
$$

Consider the first summand on the right-hand side of the inequality in (6.1.1). Let $\delta$ be a gauge defined in $[a, b]$ corresponding to $\varepsilon>0$ in the definition of the integral $\int_{a}^{b} D\left[F_{k}\left(\psi_{k}(\tau), s\right)-F_{k}\left(\psi_{0}(\tau), s\right)\right]$ and let $\left(\tau_{i},\left[s_{i-1}, s_{i}\right]\right)_{1 \leq i \leq p}$ be a $\delta$-fine tagged partition of $[a, b]$. Then,

$$
\begin{gathered}
\left\|\int_{a}^{b} D\left[F_{k}\left(\psi_{k}(\tau), s\right)-F_{k}\left(\psi_{0}(\tau), s\right)\right]\right\| \leq \| \int_{a}^{b} D\left[F_{k}\left(\psi_{k}(\tau), s\right)-F_{k}\left(\psi_{0}(\tau), s\right)\right]- \\
-\sum_{i=1}^{p}\left[\left(F_{k}\left(\psi_{k}\left(\tau_{i}\right), s_{i}\right)-F_{k}\left(\psi_{k}\left(\tau_{i}\right), s_{i-1}\right)\right)-\left(F_{k}\left(\psi_{0}\left(\tau_{i}\right), s_{i}\right)-F_{k}\left(\psi_{0}\left(\tau_{i}\right), s_{i-1}\right)\right)\right] \|+ \\
\left\|\sum_{i=1}^{p}\left[\left(F_{k}\left(\psi_{k}\left(\tau_{i}\right), s_{i}\right)-F_{k}\left(\psi_{k}\left(\tau_{i}\right), s_{i-1}\right)\right)-\left(F_{k}\left(\psi_{0}\left(\tau_{i}\right), s_{i}\right)-F_{k}\left(\psi_{0}\left(\tau_{i}\right), s_{i-1}\right)\right)\right]\right\| \leq \\
<\varepsilon+\sum_{i=1}^{p}\left\|F_{k}\left(\psi_{k}\left(\tau_{i}\right), s_{i}\right)-F_{k}\left(\psi_{k}\left(\tau_{i}\right), s_{i-1}\right)-F_{k}\left(\psi_{0}\left(\tau_{i}\right), s_{i}\right)+F_{k}\left(\psi_{0}\left(\tau_{i}\right), s_{i-1}\right)\right\| \leq \\
\leq \varepsilon+\sum_{i=1}^{p}\left|\psi_{k}\left(\tau_{i}\right)-\psi_{0}\left(\tau_{i}\right)\right|\left[h_{k}\left(s_{i}\right)-h_{k}\left(s_{i-1}\right)\right] \leq \\
\leq \varepsilon+\left\|\psi_{k}-\psi_{0}\right\|_{\infty} \sum_{i=1}^{p}\left[h_{k}\left(s_{i}\right)-h_{k}\left(s_{i-1}\right)\right] \leq \\
\leq \varepsilon+\varepsilon\left[h_{k}(b)-h_{k}(a)\right]=\varepsilon\left(1+\left[h_{k}(b)-h_{k}(a)\right]\right) \leq \varepsilon(1+c)
\end{gathered}
$$

For the second and fourth summands on the right-hand side of (6.1.1), we can show analogously that

$$
\left\|\int_{a}^{b} D\left[F_{k}\left(\psi_{0}(\tau), s\right)-F_{k}(y(\tau), s)\right]\right\|<\varepsilon\left(1+\left[h_{k}(b)-h_{k}(a)\right]\right) \leq \varepsilon(1+c)
$$

and

$$
\left\|\int_{a}^{b} D\left[F_{0}(y(\tau), s)-F_{0}\left(\psi_{0}(\tau), s\right)\right]\right\|<\varepsilon\left(1+\left[h_{0}(b)-h_{0}(a)\right]\right) \leq \varepsilon(1+c) .
$$

Thus,

$$
\left\|\int_{a}^{b} D F_{k}\left(\psi_{k}(\tau), s\right)-\int_{a}^{b} D F_{0}\left(\psi_{0}(\tau), s\right)\right\|<
$$




$$
<3 \varepsilon(1+c)+\left\|\int_{a}^{b} D\left[F_{k}(y(\tau), s)-F_{0}(y(\tau), s)\right]\right\|
$$

Now, consider the integral $\int_{a}^{b} D\left[F_{k}(y(\tau), s)-F_{0}(y(\tau), s)\right]$. Since $y:[a, b] \rightarrow X$ is a step function, there is a finite numbers $p \in \mathbb{N}$ of points $a=r_{0}<r_{1}<r_{2}<\ldots<r_{p-1}<r_{p}=b$ such that for $\tau \in\left(r_{j-1}, r_{j}\right), j=1,2, \ldots, p$, we have $y(\tau)=c_{j} \in X$ ( $y$ assumes a constant value $c_{j}$ in each open interval $\left.\left(r_{j-1}, r_{j}\right), j=1,2, \ldots\right)$. In this case, an explicit formula for the integral $\int_{a}^{b} D F_{k}(y(\tau), s)$, for every $k=0,1,2, \ldots$, can be given, namely,

$$
\int_{a}^{b} D F_{k}(y(\tau), s)=\sum_{j=1}^{p} \int_{r_{j-1}}^{r_{j}} D F_{k}(y(\tau), s)
$$

and using the Hake-type theorem and a properly chosen partition of $\left[r_{j-1}, r_{j}\right]$, we obtain

$$
\begin{gathered}
\int_{r_{j-1}}^{r_{j}} D F_{k}(y(\tau), t)=F_{k}\left(c_{j}, r_{j}^{-}\right)-F_{k}\left(c_{j}, r_{j-1}^{+}\right)+F_{k}\left(y\left(r_{j-1}\right), r_{j-1}^{+}\right)- \\
F_{k}\left(y\left(r_{j-1}\right), r_{j-1}\right)-F_{k}\left(y\left(r_{j}\right), r_{j}^{-}\right)+F_{k}\left(y\left(r_{j}\right), r_{j}\right) .
\end{gathered}
$$

Looking at the difference for the sums on the right-hand side for $F_{k}$ and $F_{0}$ in the last equality and using hypotheses and the fact that $F_{k}$ converges pointwisely to $F_{0}$, we get

$$
\lim _{k \rightarrow \infty} \int_{r_{j-1}}^{r_{j}} D\left[F_{k}(y(\tau), t)-F_{0}(y(\tau), t)\right]=0,
$$

by the hypotheses.

Since $\varepsilon>0$ can be taken arbitrarily small in (6.1.2), we obtain the desired result.

In what follows, we prove a result on continuous dependence of solution on parameters for generalized ODEs. It is a generalization of Theorem 2.4 found in [45]. Though we follow the same ideas presented in [45], we give a different proof here. Instead of the auxiliary results from [45], we employ Lemma 6.1 .

Theorem 6.2. Let $X$ be a Banach space, $O \subset X$ an open set, and $h_{k}:[a, b] \rightarrow \mathbb{R}, k=$ $1,2, \ldots$, a sequence of nondecreasing left-continuous functions such that $h_{k}(b)-h_{k}(a) \leq c$ for some $c>0$ and every $k \in \mathbb{N}_{0}$. Assume that for every $k=1,2, \ldots, F_{k}: O \times[a, b] \rightarrow X$ belongs to the class $\mathcal{F}\left(O \times[a, b], h_{k}\right)$, and that

$$
\lim _{k \rightarrow \infty} F_{k}(x, t)=F_{0}(x, t), \quad x \in O, t \in[a, b]
$$




$$
\lim _{k \rightarrow \infty} F_{k}\left(x, t^{+}\right)=F_{0}\left(x, t^{+}\right) \quad x \in O, t \in[a, b) .
$$

For every $k=1,2, \ldots$, let $x_{k}:[a, b] \rightarrow O$ be a solution of the generalized ordinary differential equation

$$
\frac{\mathrm{d} x}{\mathrm{~d} \tau}=D F_{k}(x, t)
$$

If there exists a function $x_{0}:[a, b] \rightarrow O$ such that

$$
\lim _{k \rightarrow \infty} x_{k}(t)=x_{0}(t) \text { uniformly for } t \in[a, b],
$$

then $x_{0}$ is a solution of

$$
\frac{\mathrm{d} x}{\mathrm{~d} \tau}=D F_{0}(x, t), \quad t \in[a, b]
$$

Proof. Since the functions $x_{k}:[a, b] \rightarrow O$ are regulated and $x_{k}$ tends uniformly to $x_{0}$ on $[a, b]$ when $k \rightarrow \infty$ then $x_{0}$ is a regulated function on $[a, b]$. Hence, the integral $\int_{t_{1}}^{t_{2}} D F_{0}\left(x_{0}(\tau), t\right)$ exists for every $t_{1}, t_{2} \in[a, b]$. ( See Lemma 2.14).

By the definition of solution of (6.1.5), we have

$$
x_{k}\left(t_{2}\right)-x_{k}\left(t_{1}\right)=\int_{t_{1}}^{t_{2}} D F_{k}\left(x_{k}(\tau), t\right)
$$

for every $k=1,2, \ldots$ Using (6.1.3) and (6.1.4), we obtain, by Lemma 6.1,

$$
\lim _{k \rightarrow \infty} \int_{t_{1}}^{t_{2}} D F_{k}\left(x_{k}(\tau), t\right)=\int_{t_{1}}^{t_{2}} D F_{0}\left(x_{0}(\tau), t\right),
$$

which implies the equality

$$
x_{0}\left(t_{2}\right)-x_{0}\left(t_{1}\right)=\int_{t_{1}}^{t_{2}} D F_{0}\left(x_{0}(\tau), t\right)
$$

for every $t_{1}, t_{2} \in[a, b]$ and, hence, $x_{0}$ is a solution of (6.1.6) on $[a, b]$.

The next result concerns continuous dependence of solution on parameters for generalized ODEs in Banach spaces. Such result generalizes Theorems 8.6 and 8.8 from [94], Theorem 8.1 from [4] and Theorem 2.5 from [46]. In order to prove it, we inspired ourselves in some ideas from these last two theorems. This is a new result and is contained in [32].

Theorem 6.3. Consider a sequence of functions $F_{k}: \Omega \rightarrow X$ such that $F_{k} \in \mathcal{F}\left(\Omega, h_{k}\right)$, $k=0,1,2, \ldots$. Assume that 
(1) The sequence of functions $\left\{h_{k}\right\}_{k=0}^{\infty}, h_{k}:[a, b] \rightarrow \mathbb{R}$, is equiregulated;

(2) For every $k=1,2, \ldots$, the function $h_{k}$ is nondecreasing and left-continuous satisfying $h_{k}(b)-h_{k}(a) \leq c$ for some $c>0$;

(3) For every $(x, t) \in \Omega$, suppose that

$$
\lim _{k \rightarrow \infty} F_{k}(x, t)=F_{0}(x, t)
$$

and for each $k=1,2, \ldots, x_{k}$ is a solution of the generalized ordinary differential equation

$$
\frac{d x}{d \tau}=D F_{k}(x(\tau), t)
$$

on the interval $[a, b]$ and

$$
\lim _{k \rightarrow \infty} x_{k}(t)=x_{0}(t)
$$

for every $t \in[a, b]$. Then

(i) $\left\|x_{0}\left(s_{2}\right)-x_{0}\left(s_{1}\right)\right\| \leq\left|h_{0}\left(s_{2}\right)-h_{0}\left(s_{1}\right)\right|$ for every $s_{1}, s_{2} \in[a, b]$;

(ii) $\lim _{k \rightarrow \infty} x_{k}(t)=x_{0}(t)$ uniformly on $[a, b]$;

(iii) $x_{0}$ is a solution on $[a, b]$ of the generalized ordinary differential equation

$$
\frac{d x}{d \tau}=D F_{0}(x(\tau), t)
$$

Proof. Since for every $k=1,2, \ldots, h_{k}$ is a nondecreasing function, the set $\left\{h_{k}(t) ; t \in[a, b]\right\}$ is bounded. From the fact that the sequence $\left\{h_{k}\right\}_{k=1}^{\infty}$ is equiregulated and by Corollary 1.11, there exists a subsequence $\left\{h_{n_{k}}\right\}_{k=1}^{\infty}$ which converges uniformly. Without loss of generality, we denote this subsequence by $\left\{h_{k}\right\}_{k=1}^{\infty}$. Let $h_{0}:[a, b] \rightarrow \mathbb{R}$ be the function such that $\left\{h_{k}\right\}_{k=1}^{\infty}$ converges uniformly.

Also, since $\left\{h_{k}\right\}_{k=1}^{\infty}$ is a sequence of nondecreasing functions, it follows that $h_{0}$ is a nondecreasing function.

Assume that $a<s_{1}<s_{2}<b$. For every $k=1,2, \ldots$, we have, by Lemma 2.15, the following inequality

$$
\left\|x_{k}\left(s_{2}\right)-x_{k}\left(s_{1}\right)\right\| \leq\left|h_{k}\left(s_{2}\right)-h_{k}\left(s_{2}\right)\right| .
$$

Applying the limit when $k \rightarrow \infty$ in (6.1.10), we obtain, by equation (6.1.8),

$$
\left\|x_{0}\left(s_{2}\right)-x_{0}\left(s_{1}\right)\right\| \leq\left|h_{0}\left(s_{2}\right)-h_{0}\left(s_{1}\right)\right|
$$


for every $s_{1}, s_{2} \in[a, b]$ and (i) follows immediately.

Now, let us prove item (ii). By Lemma 2.15, we have

$$
\left\|x_{k}\left(s_{2}\right)-x_{k}\left(s_{1}\right)\right\| \leq\left|h_{k}\left(s_{2}\right)-h_{k}\left(s_{1}\right)\right|, \quad s_{1}, s_{2} \in[a, b] .
$$

Moreover, since the sequence of functions $\left\{h_{k}\right\}_{k=1}^{\infty}$ is equiregulated, it follows from (6.1.11) and by Corollary 1.17, that $x_{k}$ converges uniformly to $x_{0}$ on $[a, b]$.

Now, let us prove item (iii). By the definition of solution of the generalized ODE (6.1.7), we have, for each $k=1,2, \ldots$,

$$
x_{k}\left(s_{2}\right)-x_{k}\left(s_{1}\right)=\int_{s_{1}}^{s_{2}} D F_{k}\left(x_{k}(\tau), s\right),
$$

for every $s_{1}, s_{2} \in[a, b]$.

Note that, for every $k=0,1,2, \ldots, F_{k} \in \mathcal{F}\left(\Omega, h_{k}\right)$. Also, $\left\{h_{k}\right\}_{k=1}^{\infty}$ is an equiregulated sequence of functions and $F_{k}$ converges pointwisely to $F_{0}$, it follows from Corollary 1.17 that the sequence of functions $\left\{F_{k}\right\}_{k=1}^{\infty}$ converges uniformly to $F_{0}$. Then, by Lemma 1.15, we have

$$
F_{k}\left(x, t^{+}\right) \stackrel{k \rightarrow \infty}{\rightarrow} F_{0}\left(x, t^{-}\right)
$$

Thus, all hypotheses from Lemma 6.1 are satisfied. Hence,

$$
\left\|\int_{s_{1}}^{s_{2}} D F_{k}\left(x_{k}(\tau), s\right)-\int_{s_{1}}^{s_{2}} D F_{0}\left(x_{0}(\tau), s\right)\right\| \stackrel{k \rightarrow \infty}{\rightarrow} 0
$$

for every $s_{1}, s_{2} \in[a, b]$.

Using (6.1.12), we have

$$
\begin{gathered}
\left\|x_{0}\left(s_{2}\right)-x_{0}\left(s_{1}\right)-\int_{s_{1}}^{s_{2}} D F_{0}\left(x_{0}(\tau), s\right)\right\| \leq \\
\leq\left\|x_{k}\left(s_{2}\right)-x_{0}\left(s_{2}\right)\right\|+\left\|x_{k}\left(s_{1}\right)-x_{0}\left(s_{1}\right)\right\|+\left\|\int_{s_{1}}^{s_{2}} D F_{k}\left(x_{k}(\tau), s\right)-\int_{s_{1}}^{s_{2}} D F_{0}\left(x_{0}(\tau), s\right)\right\| .
\end{gathered}
$$

Then, as $k \rightarrow \infty$, we obtain

$$
x_{0}\left(s_{2}\right)-x_{0}\left(s_{1}\right)=\int_{s_{1}}^{s_{2}} D F_{0}\left(x_{0}(\tau), s\right)
$$

for every $s_{1}, s_{2} \in[a, b]$, and the desired result follows. 
In the sequel, we prove a continuous dependence on parameters for measure functional differential equations. Such result is original and it can be found in [36].

Theorem 6.4. Assume that $X$ is a closed subspace of $G\left(\left[t_{0}-r, t_{0}+\sigma\right], \mathbb{R}^{n}\right), O$ is an open subset of $X$ with the prolongation property, $P=\left\{y_{t} ; y \in O, t \in\left[t_{0}, t_{0}+\sigma\right]\right\}, g:\left[t_{0}, t_{0}+\right.$ $\sigma] \rightarrow \mathbb{R}$ is a nondecreasing and left-continuous function, and $f_{k}: P \times\left[t_{0}, t_{0}+\sigma\right] \rightarrow \mathbb{R}^{n}$, $k=1,2, \ldots$, is a sequence of functions which satisfy the following conditions:

1. The integral $\int_{t_{0}}^{t_{0}+\sigma} f_{k}\left(y_{s}, s\right) \mathrm{d} g(s)$ exists for every $k=1,2, \ldots$ and every $y \in O$.

2. There exists a constant $M>0$ such that

$$
\left|\int_{u_{1}}^{u_{2}} f_{k}\left(y_{s}, s\right) \mathrm{d} g(s)\right| \leq M\left[g\left(u_{1}\right)-g\left(u_{2}\right)\right]
$$

for every $k=1,2, \ldots, y \in O$ and $u_{1}, u_{2} \in\left[t_{0}, t_{0}+\sigma\right]$.

3. There exists a constant $L>0$ such that

$$
\left|\int_{u_{1}}^{u_{2}}\left[f_{k}\left(y_{s}, s\right)-f_{k}\left(z_{s}, s\right)\right] \mathrm{d} g(s)\right| \leq L \int_{u_{1}}^{u_{2}}\left\|y_{s}-z_{s}\right\|_{\infty} \mathrm{d} g(s)
$$

for every $k=1,2, \ldots, y, z \in O$ and $u_{1}, u_{2} \in\left[t_{0}, t_{0}+\sigma\right]$.

4. For every $y \in O$,

$$
\lim _{k \rightarrow \infty} \int_{t_{0}}^{t} f_{k}\left(y_{s}, s\right) \mathrm{d} g(s)=\int_{t_{0}}^{t} f_{0}\left(y_{s}, s\right) \mathrm{d} g(s)
$$

uniformly with respect to $t \in\left[t_{0}, t_{0}+\sigma\right]$.

5. For every $k=0,1,2, \ldots, x \in O$ and $t \in\left[t_{0}, t_{0}+\sigma\right]$, the function $F_{k}(x, t):\left[t_{0}-\right.$ $\left.r, t_{0}+\sigma\right] \rightarrow \mathbb{R}^{n}$ given by

$$
F_{k}(x, t)(\vartheta)= \begin{cases}0, & t_{0}-r \leq \vartheta \leq t_{0}, \\ \int_{t_{0}}^{\vartheta} f_{k}\left(x_{s}, s\right) \mathrm{d} g(s), & t_{0} \leq \vartheta \leq t \leq t_{0}+\sigma \\ \int_{t_{0}}^{t} f_{k}\left(x_{s}, s\right) \mathrm{d} g(s), & t \leq \vartheta \leq t_{0}+\sigma\end{cases}
$$

is an element of $X$. 
Consider a sequence of functions $\phi_{k} \in P, k=1,2, \ldots$, such that $\lim _{k \rightarrow \infty} \phi_{k}=\phi_{0}$ uniformly on $[-r, 0]$. For every $k=1,2, \ldots$, let $y_{k} \in O$ be the solution of the problem

$$
\begin{aligned}
y_{k}(t) & =y_{k}\left(t_{0}\right)+\int_{t_{0}}^{t} f_{k}\left(\left(y_{k}\right)_{s}, s\right) \mathrm{d} g(s), \quad t \in\left[t_{0}, t_{0}+\sigma\right], \\
\left(y_{k}\right)_{t_{0}} & =\phi_{k} .
\end{aligned}
$$

If there exists a function $y_{0} \in O$ such that $\lim _{k \rightarrow \infty} y_{k}=y_{0}$ on $\left[t_{0}, t_{0}+\sigma\right]$, then $y_{0}$ is a solution of the problem

$$
\begin{aligned}
y_{0}(t) & =y_{0}\left(t_{0}\right)+\int_{t_{0}}^{t} f_{0}\left(\left(y_{0}\right)_{s}, s\right) \mathrm{d} g(s), \quad t \in\left[t_{0}, t_{0}+\sigma\right], \\
\left(y_{0}\right)_{t_{0}} & =\phi_{0} .
\end{aligned}
$$

Proof. The assumptions imply that for every $x \in O, \lim _{k \rightarrow \infty} F_{k}(x, t)=F_{0}(x, t)$ uniformly with respect to $t \in\left[t_{0}, t_{0}+\sigma\right]$. By the Moore-Osgood theorem, we have $\lim _{k \rightarrow \infty} F_{k}\left(x, t^{+}\right)=$ $F_{0}\left(x, t^{+}\right)$for every $x \in O$ and $t \in\left[t_{0}, t_{0}+\sigma\right)$. Also, since $X$ is a closed subspace, we have $F_{0}(x, t) \in X$.

It follows from Lemma 4.2 that $F_{k} \in \mathcal{F}\left(O \times\left[t_{0}, t_{0}+\sigma\right], h\right)$ for every $k \in \mathbb{N}$, where

$$
h(t)=(L+M)\left(g(t)-g\left(t_{0}\right)\right), \quad t \in\left[t_{0}, t_{0}+\sigma\right] .
$$

Since $\lim _{k \rightarrow \infty} F_{k}(x, t)=F_{0}(x, t)$, we have $F_{0} \in \mathcal{F}\left(O \times\left[t_{0}, t_{0}+\sigma\right], h\right)$.

For every $k=1,2, \ldots$ and $t \in\left[t_{0}, t_{0}+\sigma\right]$, let

$$
x_{k}(t)(\vartheta)=\left\{\begin{array}{l}
y_{k}(\vartheta), \vartheta \in\left[t_{0}-r, t\right] \\
y_{k}(t), \vartheta \in\left[t, t_{0}+\sigma\right]
\end{array}\right.
$$

According to Theorem 4.5, $x_{k}$ is a solution of the generalized ordinary differential equation

$$
\frac{\mathrm{d} x}{\mathrm{~d} \tau}=D F_{k}(x, t)
$$

For $k=1,2, \ldots$ and $t_{0} \leq t_{1} \leq t_{2} \leq t_{0}+\sigma$, we have

$$
\left|y_{k}\left(t_{2}\right)-y_{k}\left(t_{1}\right)\right|=\left|\int_{t_{1}}^{t_{2}} f_{k}\left(\left(y_{k}\right)_{s}, s\right) \mathrm{d} g(s)\right| \leq M\left(g\left(t_{2}\right)-g\left(t_{1}\right)\right),
$$

where $\eta(t)=M t$ for every $t \in[0, \infty)$. Moreover, the sequence $\left\{y_{k}\left(t_{0}\right)\right\}_{k=1}^{\infty}$ is bounded. Thus, the condition 2 from Corollary 1.11 is satisfied and it follows that $\left\{y_{k}\right\}_{k=1}^{\infty}$ contains 
a subsequence which is uniformly convergent in $\left[t_{0}, t_{0}+\sigma\right]$. Without loss of generality, we can denote this subsequence again by $\left\{y_{k}\right\}_{k=1}^{\infty}$. Since $\left(y_{k}\right)_{t_{0}}=\phi_{k}$, we see that $\left\{y_{k}\right\}_{k=1}^{\infty}$ is in fact uniformly convergent in $\left[t_{0}-r, t_{0}+\sigma\right]$.

By the definition of $x_{k}$, we have

$$
\lim _{k \rightarrow \infty} x_{k}(t)=x_{0}(t)
$$

uniformly with respect to $t \in\left[t_{0}, t_{0}+\sigma\right]$. It follows from Theorem 6.2 that $x_{0}$ is a solution of

$$
\frac{\mathrm{d} x}{\mathrm{~d} \tau}=D F_{0}(x, t)
$$

on $\left[t_{0}, t_{0}+\sigma\right]$. The proof is finished by applying Theorem 4.6, which guarantees that $y_{0}$ satisfies

$$
\begin{aligned}
y_{0}(t) & =y_{0}\left(t_{0}\right)+\int_{t_{0}}^{t} f_{0}\left(\left(y_{0}\right)_{s}, s\right) \mathrm{d} g(s), \quad t \in\left[t_{0}, t_{0}+\sigma\right] \\
\left(y_{0}\right)_{t_{0}} & =\phi_{0}
\end{aligned}
$$

and we have the desired result.

Remark 6.5. We remind the reader that although assumption 5 in the previous theorem looks complicated, it is automatically satisfied if either $g(t)=t$, for every $t \in\left[t_{0}, t_{0}+\sigma\right]$ and $X=C\left(\left[t_{0}-r, t_{0}+\sigma\right], \mathbb{R}^{n}\right)$, or if $X=G\left(\left[t_{0}-r, t_{0}+\sigma\right], \mathbb{R}^{n}\right)$. See Remark 5.5.

Using the Theorem 6.4, we prove a result on continuous dependence of solution on parameters for functional dynamic equations on time scales. Such result is original and can be found in [36].

Theorem 6.6. Let $\left[t_{0}-r, t_{0}+\sigma\right]_{\mathbb{T}}$ be a time scale interval, $t_{0} \in \mathbb{T}, B \subset \mathbb{R}^{n}$ be open, $C=C\left(\left[t_{0}-r, t_{0}+\sigma\right]_{\mathbb{T}}, B\right), P=\left\{y_{t}^{*} ; y \in C, t \in\left[t_{0}, t_{0}+\sigma\right]\right\}$. Consider a sequence of functions $f_{k}: P \times\left[t_{0}, t_{0}+\sigma\right]_{\mathbb{T}} \rightarrow \mathbb{R}^{n}, k=1,2, \ldots$, such that the following conditions are satisfied:

1. For every $y \in C$ and $k=1,2, \ldots$, the function $t \mapsto f_{k}\left(y_{t}^{*}, t\right)$ is rd-continuous on $\left[t_{0}, t_{0}+\sigma\right]_{\mathbb{T}}$.

2. There exists a constant $M>0$ such that

$$
\left|\int_{u_{1}}^{u_{2}} f_{k}(y, s) \Delta s\right| \leq M\left(u_{1}-u_{2}\right)
$$


for every $k=1,2, \ldots, y \in P$ and $u_{1}, u_{2} \in\left[t_{0}-r, t_{0}+\sigma\right]_{\mathbb{T}}$.

3. There exists a constant $L>0$ such that

$$
\left|\int_{u_{1}}^{u_{2}}\left[f_{k}(y, s)-f_{k}(z, s)\right] \Delta s\right| \leq L \int_{u_{1}}^{u_{2}}\|y-z\| \Delta s
$$

for every $k=1,2, \ldots, y, z \in P$ and $u_{1}, u_{2} \in\left[t_{0}-r, t_{0}+\sigma\right]_{\mathbb{T}}$.

4. For every $y \in C$,

$$
\lim _{k \rightarrow \infty} \int_{t_{0}}^{t} f_{k}\left(y_{s}^{*}, s\right) \Delta s=\int_{t_{0}}^{t} f_{0}\left(y_{s}^{*}, s\right) \Delta s
$$

uniformly with respect to $t \in\left[t_{0}, t_{0}+\sigma\right]_{\mathbb{T}}$.

Assume that $\phi_{k} \in C\left(\left[t_{0}-r, t_{0}\right]_{\mathbb{T}}, B\right), k=1,2, \ldots$, is a sequence of functions such that

$$
\lim _{k \rightarrow \infty} \phi_{k}=\phi_{0} \quad \text { uniformly on }\left[t_{0}-r, t_{0}\right]_{\mathbb{T}} \text {. }
$$

For every $k=1,2, \ldots$, let $y_{k} \in C$ be solution of functional dynamic equation on time scales

$$
\begin{aligned}
y_{k}^{\Delta}(t) & =f_{k}\left(\left(y_{k}^{*}\right)_{t}, t\right), \quad t \in\left[t_{0}, t_{0}+\sigma\right]_{\mathbb{T}}, \\
y_{k}(t) & =\phi_{k}(t), \quad t \in\left[t_{0}-r, t_{0}\right]_{\mathbb{T}} .
\end{aligned}
$$

If there exists a function $y_{0} \in C$ such that $\lim _{k \rightarrow \infty} y_{k}=y_{0}$ on $\left[t_{0}, t_{0}+\sigma\right]_{\mathbb{T}}$, then $y_{0}$ is a solution of functional dynamic equation on time scales

$$
\begin{aligned}
y_{0}^{\Delta}(t) & =f_{0}\left(\left(y_{0}^{*}\right)_{s}, s\right), \quad t \in\left[t_{0}, t_{0}+\sigma\right]_{\mathbb{T}}, \\
y_{0}(t) & =\phi_{0}(t), \quad t \in\left[t_{0}-r, t_{0}\right]_{\mathbb{T}} .
\end{aligned}
$$

Proof. Let $X=\left\{y^{*} ; y \in C\left(\left[t_{0}-r, t_{0}+\sigma\right]_{\mathbb{T}}, \mathbb{R}^{n}\right)\right\}, O=\left\{y^{*} ; y \in C\right\}$, and $g(t)=t^{*}$ for every $t \in\left[t_{0}, t_{0}+\sigma\right]$. Note that $O$ is an open subset of $X$ and has the prolongation property. Further, let $f_{k}^{*}(y, t)=f_{k}\left(y, t^{*}\right)$ for every $k=1,2, \ldots, y \in P$ and $t \in\left[t_{0}, t_{0}+\sigma\right]$. Consider an arbitrary $y \in O$ and $k=1,2, \ldots$. Since $t \mapsto f_{k}\left(y_{t}^{*}, t\right)$ is rd-continuous on $\left[t_{0}, t_{0}+\sigma\right]_{\mathbb{T}}$, the integral $\int_{t_{0}}^{t_{0}+\sigma} f_{k}\left(y_{t}, t\right) \Delta t$ exists. Using Theorem 4.11 and Theorem 4.12, we have

$$
\int_{t_{0}}^{t_{0}+\sigma} f_{k}\left(y_{t}, t\right) \Delta t=\int_{t_{0}}^{t_{0}+\sigma} f_{k}\left(y_{t^{*}}, t^{*}\right) \mathrm{d} g(t)=\int_{t_{0}}^{t_{0}+\sigma} f_{k}\left(y_{t}, t^{*}\right) \mathrm{d} g(t)=\int_{t_{0}}^{t_{0}+\sigma} f_{k}^{*}\left(y_{t}, t\right) \mathrm{d} g(t),
$$


that is, the last integral exists. Using Theorem 4.11 again, we obtain

$$
\lim _{k \rightarrow \infty} \int_{t_{0}}^{t} f_{k}^{*}\left(y_{s}, s\right) \mathrm{d} g(s)=\lim _{k \rightarrow \infty} \int_{t_{0}}^{t^{*}} f_{k}\left(y_{s}, s\right) \Delta s=\int_{t_{0}}^{t^{*}} f_{0}\left(y_{s}, s\right) \Delta s=\int_{t_{0}}^{t} f_{0}^{*}\left(y_{s}, s\right) \mathrm{d} g(s)
$$

uniformly with respect to $t \in\left[t_{0}, t_{0}+\sigma\right]$. Further, it is clear that $\lim _{k \rightarrow \infty} y_{k}^{*}=y_{0}^{*}$ on $\left[t_{0}, t_{0}+\sigma\right]$, and $\lim _{k \rightarrow \infty}\left(\phi_{k}^{*}\right)_{t_{0}}=\left(\phi_{0}^{*}\right)_{t_{0}}$ uniformly on $[-r, 0]$. By Theorem 4.13, we have

$$
\begin{aligned}
y_{k}^{*}(t) & =y_{k}^{*}\left(t_{0}\right)+\int_{t_{0}}^{t} f_{k}\left(\left(y_{k}^{*}\right)_{s}, s^{*}\right) \mathrm{d} g(s), \quad t \in\left[t_{0}, t_{0}+\sigma\right] \\
\left(y_{k}^{*}\right)_{t_{0}} & =\left(\phi_{k}^{*}\right)_{t_{0}}
\end{aligned}
$$

for every $k=1,2, \ldots$. The functions $f_{k}^{*}, y_{k}^{*}$ and $\left(\phi_{k}^{*}\right)_{t_{0}}, k=1,2, \ldots$, satisfy the assumptions of Theorem 6.4, and we conclude that

$$
\begin{aligned}
y_{0}^{*}(t) & =y_{0}^{*}\left(t_{0}\right)+\int_{t_{0}}^{t} f_{0}\left(\left(y_{0}^{*}\right)_{s}, s^{*}\right) \mathrm{d} g(s), \quad t \in\left[t_{0}, t_{0}+\sigma\right] \\
\left(y_{0}^{*}\right)_{t_{0}} & =\left(\phi_{0}^{*}\right)_{t_{0}} .
\end{aligned}
$$

By Theorem 4.13, it follows that $y_{0}:\left[t_{0}-r, t_{0}+\sigma\right]_{\mathbb{T}} \rightarrow \mathbb{R}^{n}$ satisfies

$$
\begin{aligned}
y_{0}^{\Delta}(t) & =f_{0}\left(\left(y_{0}^{*}\right)_{s}, s\right), \quad t \in\left[t_{0}, t_{0}+\sigma\right]_{\mathbb{T}}, \\
y_{0}(t) & =\phi_{0}(t), \quad t \in\left[t_{0}-r, t_{0}\right]_{\mathbb{T}}
\end{aligned}
$$

and we obtain the desired result.

In the sequel, we prove a result on continuous dependence of solutions on parameters for impulsive measure functional differential equations. This is a new result and can be found in [37].

Theorem 6.7. Assume that $X=G\left(\left[t_{0}-r, t_{0}+\sigma\right], \mathbb{R}^{n}\right), B \subset \mathbb{R}^{n}$ is an open set, $O=$ $G\left(\left[t_{0}-r, t_{0}+\sigma\right], B\right), P=G([-r, 0], B), m \in \mathbb{N}, t_{1}, t_{2}, \ldots, t_{m}$ are moments of impulses such that $t_{0} \leq t_{1}<t_{2}<\ldots<t_{m}<t_{0}+\sigma, g:\left[t_{0}, t_{0}+\sigma\right] \rightarrow \mathbb{R}$ is a nondecreasing and left-continuous function which is continuous at $t_{1}, \ldots, t_{m}$. Finally, for every $k=1,2, \ldots$, let $f_{k}: P \times\left[t_{0}, t_{0}+\sigma\right] \rightarrow \mathbb{R}^{n}$ and $I_{1}^{k}, \ldots, I_{m}^{k}: B \rightarrow \mathbb{R}^{n}$ be functions which satisfy the following conditions:

1. The integral $\int_{t_{0}}^{t_{0}+\sigma} f_{k}\left(y_{s}, s\right) \mathrm{d} g(s)$ exists for every $k=1,2, \ldots, y \in O$. 
2. There exists a constant $M_{1}>0$ such that

$$
\left|\int_{u_{1}}^{u_{2}} f_{k}\left(y_{s}, s\right) \mathrm{d} g(s)\right| \leq M_{1}\left[g\left(u_{2}\right)-g\left(u_{1}\right)\right]
$$

whenever $k=1,2, \ldots, t_{0} \leq u_{1} \leq u_{2} \leq t_{0}+\sigma$ and $y \in O$.

3. There exists a constant $L_{1}>0$ such that

$$
\left|\int_{u_{1}}^{u_{2}}\left(f_{k}\left(y_{s}, s\right)-f_{k}\left(z_{s}, s\right)\right) \mathrm{d} g(s)\right| \leq L_{1} \int_{u_{1}}^{u_{2}}\left\|y_{s}-z_{s}\right\|_{\infty} \mathrm{d} g(s),
$$

whenever $k=1,2, \ldots, t_{0} \leq u_{1} \leq u_{2} \leq t_{0}+\sigma$ and $y, z \in O$.

4. For every $y \in O$,

$$
\lim _{k \rightarrow \infty} \int_{t_{0}}^{t} f_{k}\left(y_{s}, s\right) \mathrm{d} g(s)=\int_{t_{0}}^{t} f_{0}\left(y_{s}, s\right) \mathrm{d} g(s)
$$

uniformly with respect to $t \in\left[t_{0}, t_{0}+\sigma\right]$.

5. There exists a constant $M_{2}>0$ such that

$$
\left|I_{j}^{k}(x)\right| \leq M_{2}
$$

for every $j=1, \ldots, m, k=1,2, \ldots$ and $x \in B$.

6. There exists a constant $L_{2}>0$ such that

$$
\left|I_{j}^{k}(x)-I_{j}^{k}(y)\right| \leq L_{2}|x-y|
$$

for every $j=1, \ldots, m, k=1,2, \ldots$ and $x, y \in B$.

7. For every $y \in B$ and $j=1, \ldots, m, \lim _{k \rightarrow \infty} I_{j}^{k}(y)=I_{j}^{0}(y)$.

Consider functions $\phi_{k} \in P, k=1,2, \ldots$, such that $\lim _{k \rightarrow \infty} \phi_{k}=\phi_{0}$ uniformly on $[-r, 0]$. For every $k=1,2, \ldots$, let $y_{k} \in O$ be solutions of the problem

$$
\begin{aligned}
y_{k}(t) & =y_{k}\left(t_{0}\right)+\int_{t_{0}}^{t} f_{k}\left(\left(y_{k}\right)_{s}, s\right) \mathrm{d} g(s)+\sum_{\substack{j \in\{1, \ldots, m\}, t_{k}<t}} I_{j}^{k}\left(y_{k}\left(t_{j}\right)\right), \\
\left(y_{k}\right)_{t_{0}} & =\phi_{k},
\end{aligned}
$$


such that $\lim _{k \rightarrow \infty} y_{k}=y_{0} \in O$. Then $y_{0}$ satisfies the following impulsive equation:

$$
\begin{aligned}
y_{0}(t) & =y_{0}\left(t_{0}\right)+\int_{t_{0}}^{t} f_{0}\left(\left(y_{0}\right)_{s}, s\right) \mathrm{d} g(s)+\sum_{\substack{j \in\{1, \ldots, m\} \\
t_{j}<t}} I_{k}^{0}\left(y_{0}\left(t_{j}\right)\right), \\
\left(y_{0}\right)_{t_{0}} & =\phi_{0} .
\end{aligned}
$$

Proof. We already know that (6.1.13) and (6.1.14) imply

$$
\begin{aligned}
y_{k}(t) & =y_{k}\left(t_{0}\right)+\int_{t_{0}}^{t} \tilde{f}_{k}\left(\left(y_{k}\right)_{s}, s\right) \mathrm{d} \tilde{g}(s), \quad t \in\left[t_{0}, t_{0}+\sigma\right] \\
\left(y_{k}\right)_{t_{0}} & =\phi_{k},
\end{aligned}
$$

where the construction of $\tilde{f}_{k}$ and $\tilde{g}$ is described in Theorem 4.16. Since $g$ is nondecreasing and left-continuous, $\tilde{g}$ must have the same properties. For every $t \in\left[t_{0}, t_{0}+\sigma\right]$, we have

$$
\begin{gathered}
\lim _{k \rightarrow \infty} \int_{t_{0}}^{t} \tilde{f}_{k}\left(y_{s}, s\right) \mathrm{d} \tilde{g}(s)=\lim _{k \rightarrow \infty} \int_{t_{0}}^{t} f_{k}\left(y_{s}, s\right) \mathrm{d} g(s)+\lim _{k \rightarrow \infty} \sum_{\substack{j \in\{1, \ldots, m\}, t_{j}<t}} I_{j}^{k}\left(y\left(t_{j}\right)\right)= \\
=\int_{t_{0}}^{t} f_{0}\left(y_{s}, s\right) \mathrm{d} g(s)+\sum_{\substack{j \in\{1, \ldots, m\} \\
t_{j}<t}} I_{j}^{0}\left(y\left(t_{j}\right)\right)=\int_{t_{0}}^{t} \tilde{f}_{0}\left(y_{s}, s\right) \mathrm{d} \tilde{g}(s),
\end{gathered}
$$

where the convergence is uniform with respect to $t \in\left[t_{0}, t_{0}+\sigma\right]$.

Using these facts and Lemma 4.18, we see that the functions $\tilde{f}_{k}, \tilde{g}$, and $\phi_{k}$ satisfy all hypotheses of Theorem 6.4. Consequently,

$$
\begin{aligned}
y_{0}(t) & =y_{0}\left(t_{0}\right)+\int_{t_{0}}^{t} \tilde{f}_{0}\left(\left(y_{0}\right)_{s}, s\right) \mathrm{d} \tilde{g}(s), \quad t \in\left[t_{0}, t_{0}+\sigma\right] \\
\left(y_{0}\right)_{t_{0}} & =\phi_{0}
\end{aligned}
$$

The proof is finished by applying Theorem 4.16, which implies that $y_{0}$ satisfies (6.1.15) and (6.1.16).

The last result in this section is a result on continuous dependence of solution on parameters for impulsive functional dynamic equations on time scales. This is also an original result and can be found in [37].

Theorem 6.8. Assume that $\left[t_{0}-r, t_{0}+\sigma\right]_{\mathbb{T}}$ is a time scale interval, $t_{0} \in \mathbb{T}, B \subset \mathbb{R}^{n}$ is an open set, $O=G\left(\left[t_{0}-r, t_{0}+\sigma\right], B\right), P=G([-r, 0], B), m \in \mathbb{N}, t_{1}, \ldots, t_{m} \in\left[t_{0}, t_{0}+\sigma\right]_{\mathbb{T}}$ 
are right-dense points such that $t_{0} \leq t_{1}<\cdots<t_{m}<t_{0}+\sigma$. For every $k=1,2, \ldots$, let $f_{k}: P \times\left[t_{0}, t_{0}+\sigma\right]_{\mathbb{T}} \rightarrow \mathbb{R}^{n}$ and $I_{1}^{k}, \ldots, I_{m}^{k}: B \rightarrow \mathbb{R}^{n}$ be functions which satisfy the following conditions:

1. The integral $\int_{t_{0}}^{t_{0}+\sigma} f_{k}\left(y_{s}, s\right) \Delta s$ exists for every $y \in O$ and $k=1,2, \ldots$.

2. There exists a constant $M_{1}>0$ such that

$$
\left|\int_{u_{1}}^{u_{2}} f_{k}\left(y_{s}, s\right) \Delta s\right| \leq M_{1}\left(u_{2}-u_{1}\right)
$$

for every $k=1,2, \ldots, y \in O$ and $u_{1}, u_{2} \in\left[t_{0}, t_{0}+\sigma\right]_{\mathbb{T}}, u_{1} \leq u_{2}$.

3. There exists a constant $L_{1}>0$ such that

$$
\left|\int_{u_{1}}^{u_{2}}\left[f_{k}\left(y_{s}, s\right)-f_{k}\left(z_{s}, s\right)\right] \Delta s\right| \leq L_{1} \int_{u_{1}}^{u_{2}}\left\|y_{s}-z_{s}\right\|_{\infty} \Delta s
$$

for every $k=1,2, \ldots, y, z \in O$ and $u_{1}, u_{2} \in\left[t_{0}, t_{0}+\sigma\right]_{\mathbb{T}}, u_{1} \leq u_{2}$.

4. For every $y \in O$,

$$
\lim _{k \rightarrow \infty} \int_{t_{0}}^{t} f_{k}\left(y_{s}, s\right) \Delta s=\int_{t_{0}}^{t} f_{0}\left(y_{s}, s\right) \Delta s
$$

uniformly with respect to $t \in\left[t_{0}, t_{0}+\sigma\right]_{\mathbb{T}}$.

5. There exists a constant $M_{2}>0$ such that

$$
\left|I_{j}^{k}(x)\right| \leq M_{2}
$$

for every $j=1, \ldots, m, k=1,2, \ldots$ and $x \in B$.

6. There exists a constant $L_{2}>0$ such that

$$
\left|I_{j}^{k}(x)-I_{j}^{k}(y)\right| \leq L_{2}|x-y|
$$

for every $j=1, \ldots, m, k=1,2, \ldots$ and $x, y \in B$.

7. For every $x \in B$ and $j=1,2, \ldots, m, \lim _{k \rightarrow \infty} I_{j}^{k}(x)=I_{j}^{0}(x)$.

Assume that $\phi_{k} \in G\left(\left[t_{0}-r, t_{0}\right]_{\mathbb{T}}, B\right), k=1,2, \ldots$, is a sequence of functions such that

$$
\lim _{k \rightarrow \infty} \phi_{k}=\phi_{0} \quad \text { uniformly on }\left[t_{0}-r, t_{0}\right]_{\mathbb{T}} .
$$


For every $k=1,2, \ldots$, let $y_{k}:\left[t_{0}-r, t_{0}+\sigma\right]_{\mathbb{T}} \rightarrow B$ be solution of impulsive functional dynamic equation on time scales

$$
\begin{aligned}
& y_{k}(t)=y_{k}\left(t_{0}\right)+\int_{t_{0}}^{t} f_{k}\left(\left(y_{k}^{*}\right)_{s}, s\right) \Delta s+\sum_{\substack{j \in\{1, \ldots, m\}, t_{j}<t}} I_{j}^{k}\left(y_{k}\left(t_{j}\right)\right), \quad t \in\left[t_{0}, t_{0}+\sigma\right]_{\mathbb{T}}, \\
& y_{k}(t)=\phi_{k}(t), \quad t \in\left[t_{0}-r, t_{0}\right]_{\mathbb{T}} .
\end{aligned}
$$

If there exists a function $y_{0}:\left[t_{0}-r, t_{0}+\sigma\right]_{\mathbb{T}} \rightarrow B$ such that $\lim _{k \rightarrow \infty} y_{k}=y_{0}$, then $y_{0}$ satisfies the impulsive functional dynamic equation on time scales given by

$$
\left\{\begin{array}{l}
y_{0}(t)=y_{0}\left(t_{0}\right)+\int_{t_{0}}^{t} f_{0}\left(\left(y_{0}^{*}\right)_{s}, s\right) \Delta s+\sum_{\substack{j \in\{1, \ldots, m\}, t_{j}<t}} I_{j}^{0}\left(y_{0}\left(t_{j}\right)\right), \quad t \in\left[t_{0}, t_{0}+\sigma\right]_{\mathbb{T}}, \\
y_{0}(t)=\phi_{0}(t), \quad t \in\left[t_{0}-r, t_{0}\right]_{\mathbb{T}} .
\end{array}\right.
$$

Proof. Let $g(t)=t^{*}$ for every $t \in\left[t_{0}, t_{0}+\sigma\right]$. Then $g$ is a left-continuous nondecreasing function which is continuous at $t_{1}, \ldots, t_{m}$. Further, let $f_{k}^{*}(y, t)=f_{k}\left(y, t^{*}\right)$ for every $k=1,2, \ldots, y \in P$ and $t \in\left[t_{0}, t_{0}+\sigma\right]$. By Lemma 4.24, the integral $\int_{t_{0}}^{t_{0}+\sigma} f_{k}^{*}\left(y_{t}, t\right) \mathrm{d} g(t)$ exists for every $y \in O$ and $k=1,2, \ldots$. By Theorems 4.11 and 4.12 , we obtain

$$
\begin{gathered}
\lim _{k \rightarrow \infty} \int_{t_{0}}^{t} f_{k}^{*}\left(y_{s}, s\right) \mathrm{d} g(s)=\lim _{k \rightarrow \infty} \int_{t_{0}}^{t^{*}} f_{k}\left(y_{s}, s\right) \Delta s=\int_{t_{0}}^{t^{*}} f_{0}\left(y_{s}, s\right) \Delta s= \\
=\int_{t_{0}}^{t} f_{0}\left(y_{s^{*}}, s^{*}\right) \mathrm{d} g(s)=\int_{t_{0}}^{t} f_{0}^{*}\left(y_{s}, s\right) \mathrm{d} g(s),
\end{gathered}
$$

where the convergence is uniform with respect to $t \in\left[t_{0}, t_{0}+\sigma\right]$.

Further, it is clear that $\lim _{k \rightarrow \infty} y_{k}^{*}=y_{0}^{*}$ on $\left[t_{0}, t_{0}+\sigma\right]$, and $\lim _{k \rightarrow \infty} \phi_{k}^{*}=\phi_{0}^{*}$ uniformly on $\left[t_{0}-r, t_{0}\right]$. By Theorem 4.23, we have

$$
\begin{aligned}
y_{k}^{*}(t) & =y_{k}^{*}\left(t_{0}\right)+\int_{t_{0}}^{t} f_{k}^{*}\left(\left(y_{k}^{*}\right)_{s}, s\right) \mathrm{d} g(s)+\sum_{\substack{j \in\{1, \ldots, m\}, t_{j}<t}} I_{j}^{k}\left(y_{k}^{*}\left(t_{j}\right)\right), \quad t \in\left[t_{0}, t_{0}+\sigma\right], \\
\left(y_{k}^{*}\right)_{t_{0}} & =\left(\phi_{k}^{*}\right)_{t_{0}},
\end{aligned}
$$

for every $k=1,2, \ldots$. Using Lemma 4.24 , we see that all hypotheses of Theorem 6.7 are 
satisfied. Consequently,

$$
\begin{aligned}
y_{0}^{*}(t) & =y_{0}^{*}\left(t_{0}\right)+\int_{t_{0}}^{t} f_{0}^{*}\left(\left(y_{0}^{*}\right)_{s}, s\right) \mathrm{d} g(s)+\sum_{\substack{j \in\{1, \ldots, m\}, t_{j}<t}} I_{j}^{0}\left(y_{0}^{*}\left(t_{j}\right)\right), \quad t \in\left[t_{0}, t_{0}+\sigma\right], \\
\left(y_{0}^{*}\right)_{t_{0}} & =\left(\phi_{0}^{*}\right)_{t_{0}} .
\end{aligned}
$$

By Theorem 4.23, it follows that $y_{0}$ satisfies (6.1.17).

Remark 6.9. According to Remark 6.6 in [36], the assumptions of Theorem 6.4 might be modified in the following way: instead of requiring the existence of a function $y_{0} \in O$ such that $\lim _{k \rightarrow \infty} y_{k}=y_{0}$, it is enough to assume the existence of a closed set $B^{\prime} \subset B$ such that the functions $y_{k}, k=1,2, \ldots$, take values in $B^{\prime}$. Under this hypothesis, the conclusion is that $\left\{y_{k}\right\}_{k=1}^{\infty}$ has a subsequence which is uniformly convergent to a function $y_{0} \in O$ such that

$$
\begin{aligned}
y_{0}(t) & =y_{0}\left(t_{0}\right)+\int_{t_{0}}^{t} f_{0}\left(\left(y_{0}\right)_{s}, s\right) \mathrm{d} g(s), \quad t \in\left[t_{0}, t_{0}+\sigma\right] \\
\left(y_{0}\right)_{t_{0}} & =\phi_{0}
\end{aligned}
$$

Theorems 6.7 and 6.8 can be modified in a similar way.

\subsection{Continuous dependence of solutions involving vari- able time scales}

The fact that solutions of dynamic equations on time scales depend continuously on the time scales is a problem that has been investigated by several researchers. See [1],[31] and [76], for instance. In such papers, the authors prove that the sequence of solutions of the problem

$$
\left\{\begin{array}{l}
x^{\Delta}(t)=f(x, t), \quad t \in \mathbb{T}_{n}, \\
x\left(t_{0}\right)=x_{0}, \quad t_{0} \in \mathbb{T}_{n},
\end{array}\right.
$$

converges uniformly to the solution of the problem

$$
\left\{\begin{array}{l}
x^{\Delta}(t)=f(x, t), \quad t \in \mathbb{T}, \\
x\left(t_{0}\right)=x_{0}, \quad t_{0} \in \mathbb{T},
\end{array}\right.
$$


whenever $d\left(\mathbb{T}_{n}, \mathbb{T}\right) \rightarrow 0$ as $n \rightarrow \infty$, where $d\left(\mathbb{T}_{n}, \mathbb{T}\right)$ denotes the Hausdorff metric or the induced metric from the Fell topology.

Our goal is to investigate the behavior of solutions of the same initial value problems over different time scales for impulsive functional dynamic equations, that is, we prove that the sequence of solutions of the system

$$
\left\{\begin{array}{l}
x(t)=x\left(t_{0}\right)+\int_{t_{0}}^{t} f\left(x_{s}, s\right) \Delta s+\sum_{\substack{k \in\{1, \ldots, m\} \\
t_{k}<t}} I_{k}\left(x\left(t_{k}\right)\right), \quad t \in\left[t_{0}, t_{0}+\eta\right]_{\mathbb{T}_{n}}, \\
x(t)=\phi(t), \quad t \in\left[t_{0}-r, t_{0}\right]_{\mathbb{T}_{n}},
\end{array}\right.
$$

converges uniformly to the solution of the problem

$$
\left\{\begin{array}{l}
x(t)=x\left(t_{0}\right)+\int_{t_{0}}^{t} f\left(x_{s}, s\right) \Delta s+\sum_{\substack{k \in\{1, \ldots, m\} \\
t_{k}<t}} I_{k}\left(x\left(t_{k}\right)\right), \quad t \in\left[t_{0}, t_{0}+\eta\right]_{\mathbb{T}}, \\
x(t)=\phi(t), \quad t \in\left[t_{0}-r, t_{0}\right]_{\mathbb{T}}
\end{array}\right.
$$

whenever $d\left(\mathbb{T}_{n}, \mathbb{T}\right) \rightarrow 0$ as $n \rightarrow \infty$. Here, $d\left(\mathbb{T}_{n}, \mathbb{T}\right)$ denotes the Hausdorff metric. Our results apply to the Fell topology as well.

It worth mentioning that all the results presented in this section are new and they can be found in [16].

In order to obtain the continuous dependence result for impulsive functional dynamic equations on time scales involving variable time scales with these conditions, we use the correspondences between various types of equations which were presented in Chapter 4 .

Here, we introduce a different notation as the one used in the previous chapters. Instead of using the notation $t^{*}$ to denote the set $\inf \{s \in \mathbb{T} ; s \geq t\}$, we use the notation $\tilde{\sigma}(t)$ to represent it, in order to avoid any mistakes arising to the notation, since we are leading with variable time scales.

Also, we denote $\tilde{\sigma}_{n}(t)=\inf \left\{s \in \mathbb{T}_{n} ; s \geq t\right\}$. We are using the same notation as found in $[86]$.

Further, in order to guarantee the convergence of solutions, we suppose some convergence over the operator sequence $\left\{\tilde{\sigma}_{n}\right\}_{n=1}^{\infty}$. This hypothesis cannot be suppressed as shown by Examples 6.11 and 6.12 later.

In this section, we present a continuous dependence result involving variable time scales for impulsive functional dynamic equations on time scales. 
Our idea to prove a continuous dependence result for impulsive functional dynamic equations on time scales is to use the correspondence between the solutions of these equations and the solutions of impulsive measure functional differential equations (see Theorem 4.13) and the correspondence between the solutions of impulsive measure functional differential equations and the solutions of measure functional differential equations.

Let $\mathbb{T}_{n}$ be time scales for each $n \in \mathbb{N}$ with corresponding forward jumps $\sigma_{n}$ and $\tilde{\sigma}_{n}$. Assume that the distance $d\left(\mathbb{T}_{n}, \mathbb{T}\right) \rightarrow 0$ as $n \rightarrow \infty$. Here, we are considering the Hausdorff topology and Hausdorff metric in which the distance between two sets is defined by

$$
d(A, B)=\max \{\sup \{\inf \{|a-b|: b \in B\}: a \in A\}, \sup \{\inf \{|a-b|: a \in A\}: b \in B\}\}
$$

Now, our goal is to prove a result which guarantees that the sequence of solutions of the problem

$$
\left\{\begin{array}{l}
x_{n}^{\tilde{\sigma}_{n}}(t)=x_{n}^{\tilde{\sigma}_{n}}\left(t_{0}\right)+\int_{t_{0}}^{t} f\left(\left(x_{n}^{\tilde{\sigma}_{n}}\right)_{s}, s\right) \mathrm{d} \tilde{\sigma}_{n}(s), \quad t \in \mathbb{T}_{n}^{*} \\
\left(x_{n}^{\tilde{\sigma}_{n}}\right)_{t_{0}}=\phi^{\tilde{\sigma}_{n}}
\end{array}\right.
$$

converges uniformly to the solution of the problem

$$
\left\{\begin{array}{l}
x^{\tilde{\sigma}}(t)=x^{\tilde{\sigma}}\left(t_{0}\right)+\int_{t_{0}}^{t} f\left(x_{s}^{\tilde{\sigma}}, s\right) \mathrm{d} \tilde{\sigma}(s), \quad t \in \mathbb{T}^{*} \\
x_{t_{0}}^{\tilde{\sigma}}=\phi^{\tilde{\sigma}}
\end{array}\right.
$$

Thus, after proving this result, using the correspondence between impulsive measure functional differential equations and measure functional differential equations, we obtain an analogous result for measure functional differential equations with impulses and, therefore, using the other correspondence (Theorem 4.13), our main theorem concerning continuous dependence for impulsive functional dynamic equations on time scales follows as well.

Now, assume $O \subset G\left(\left[t_{0}-r, t_{0}+\eta\right], \mathbb{R}^{n}\right)$ is open, $P=\left\{y_{t} ; y \in O, t \in\left[t_{0}, t_{0}+\eta\right]\right\}$, $f: P \times\left[t_{0}, t_{0}+\eta\right] \rightarrow \mathbb{R}^{n}$, and $g:\left[t_{0}, t_{0}+\eta\right] \rightarrow \mathbb{R}$ is nondecreasing and left-continuous function.

We assume the following three conditions on the function $f: P \times\left[t_{0}, t_{0}+\eta\right] \rightarrow \mathbb{R}^{n}$ :

(A) The Kurzweil-Henstock-Stieltjes integral $\int_{t_{0}}^{t_{0}+\eta} f\left(y_{t}, t\right) \mathrm{d} g(t)$ exists for every $y \in O$.

(B) There exists a constant $M>0$ such that

$$
\left|f\left(y_{t}, t\right)\right| \leq M
$$


whenever $t_{0} \leq t \leq t_{0}+\eta$ and $y \in O$.

(C) There exists a constant $L>0$ such that

$$
\left|\int_{u_{1}}^{u_{2}}\left(f\left(y_{t}, t\right)-f\left(z_{t}, t\right)\right) \mathrm{d} g(t)\right| \leq L \int_{u_{1}}^{u_{2}}\left\|y_{t}-z_{t}\right\|_{\infty} \mathrm{d} g(t)
$$

whenever $t_{0} \leq u_{1} \leq u_{2} \leq t_{0}+\eta$ and $y, z \in O$.

Now, we are able to present our first result in this section. It is an original result and it is contained in [16].

Theorem 6.10. Suppose $f$ satisfies conditions (A), (B) and (C), and $x_{n}^{\tilde{\sigma}_{n}}$ is a solution of the system

$$
\left\{\begin{array}{l}
x_{n}^{\tilde{\sigma}_{n}}(t)=x_{n}^{\tilde{\sigma}_{n}}\left(t_{0}\right)+\int_{t_{0}}^{t} f\left(\left(x_{n}^{\tilde{\sigma}_{n}}\right)_{s}, s\right) \mathrm{d} \tilde{\sigma}_{n}(s), \quad t \in \mathbb{T}_{n}^{*} \\
\left(x_{n}^{\tilde{\sigma}_{n}}\right)_{t_{0}}=\phi^{\tilde{\sigma}_{n}}
\end{array}\right.
$$

and $x^{\tilde{\sigma}}$ is a solution of the measure functional differential equation given by

$$
\left\{\begin{array}{l}
x^{\tilde{\sigma}}(t)=x^{\tilde{\sigma}}\left(t_{0}\right)+\int_{t_{0}}^{t} f\left(x_{s}^{\tilde{\sigma}}, s\right) \mathrm{d} \tilde{\sigma}(s), \quad t \in \mathbb{T}^{*} \\
x_{t_{0}}^{\tilde{\sigma}}=\phi^{\tilde{\sigma}}
\end{array}\right.
$$

Moreover, the sequence of functions $\left\{\tilde{\sigma}_{n}\right\}_{n=1}^{\infty}$ converges uniformly to $\tilde{\sigma}$ as $n \rightarrow \infty$. Also, suppose the sequence of initial conditions $\left\{\phi^{\tilde{\sigma}_{n}}\right\}_{n=1}^{\infty}$ converges uniformly to $\phi^{\tilde{\sigma}}$ as $n \rightarrow \infty$. Then, for every $\varepsilon>0$, there exists $N>0$ sufficiently large such that, for $n>N$, we have

$$
\left|x_{n}^{\tilde{\sigma}_{n}}(t)-x^{\tilde{\sigma}}(t)\right|<\varepsilon, \quad \text { for } t \in \mathbb{T}_{n}^{*} \cap \mathbb{T}^{*}
$$

Proof. Given $\varepsilon>0$ and since the sequence of functions $\left\{\tilde{\sigma}_{n}\right\}$ converges uniformly to $\tilde{\sigma}$, there exists $N_{1}>0$ sufficiently large such that for every $n>N_{1}$, we obtain

$$
\left|\tilde{\sigma}_{n}(t)-\tilde{\sigma}(t)\right|<\varepsilon, \text { for every } t \in \mathbb{T}_{n}^{*} \cap \mathbb{T}^{*}
$$

Moreover, since the sequence of functions $\phi^{\tilde{\sigma}_{n}}$ converges uniformly to $\phi^{\tilde{\sigma}}$, there exists $N_{2}>0$ sufficiently large such that for every $n>N_{2}$, we have

$$
\left\|\phi^{\tilde{\sigma}_{n}}-\phi^{\tilde{\sigma}}\right\|_{\infty}<\varepsilon, \text { for every } t \in \mathbb{T}_{n}^{*} \cap \mathbb{T}^{*}
$$


Also, for $t \in \mathbb{T}_{n}^{*} \cap \mathbb{T}^{*}$ and $n>\max \left\{N_{1}, N_{2}\right\}$, we have

$$
\begin{aligned}
& \left|x_{n}^{\tilde{\sigma}_{n}}(t)-x^{\tilde{\sigma}}(t)\right|=\left|x_{n}^{\tilde{\sigma}_{n}}\left(t_{0}\right)-x^{\tilde{\sigma}}\left(t_{0}\right)+\int_{t_{0}}^{t} f\left(\left(x_{n}^{\tilde{\sigma}_{n}}\right)_{s}, s\right) d \tilde{\sigma}_{n}(s)-\int_{t_{0}}^{t} f\left(\left(x^{\tilde{\sigma}}\right)_{s}, s\right) d \tilde{\sigma}(s)\right| \\
& \leq\left|x_{n}^{\tilde{\sigma}_{n}}\left(t_{0}\right)-x^{\tilde{\sigma}}\left(t_{0}\right)\right|+\left|\int_{t_{0}}^{t} f\left(\left(x_{n}^{\tilde{\sigma}_{n}}\right)_{s}, s\right) d \tilde{\sigma}_{n}(s)-\int_{t_{0}}^{t} f\left(\left(x^{\tilde{\sigma}}\right)_{s}, s\right) d \tilde{\sigma}(s)\right| \\
& \leq\left\|\phi^{\tilde{\sigma}_{n}}-\phi^{\tilde{\sigma}}\right\|_{\infty}+\left|\int_{t_{0}}^{t} f\left(\left(x_{n}^{\tilde{\sigma}_{n}}\right)_{s}, s\right) d \tilde{\sigma}_{n}(s)-\int_{t_{0}}^{t} f\left(\left(x^{\tilde{\sigma}}\right)_{s}, s\right) d \tilde{\sigma}(s)\right| \\
& \leq\left\|\phi^{\tilde{\sigma}_{n}}-\phi^{\tilde{\sigma}}\right\|_{\infty}+\left|\int_{t_{0}}^{t} f\left(\left(x_{n}^{\tilde{\sigma}_{n}}\right)_{s}, s\right) d \tilde{\sigma}_{n}(s)-\int_{t_{0}}^{t} f\left(\left(x_{n}^{\tilde{\sigma}_{n}}\right)_{s}, s\right) d \tilde{\sigma}(s)\right| \\
& \quad+\left|\int_{t_{0}}^{t} f\left(\left(x_{n}^{\tilde{\sigma}_{n}}\right)_{s}, s\right) d \tilde{\sigma}(s)-\int_{t_{0}}^{t} f\left(\left(x^{\tilde{\sigma}}\right)_{s}, s\right) d \tilde{\sigma}(s)\right| \\
& \leq \varepsilon+\int_{t_{0}}^{t} M d\left[\tilde{\sigma}_{n}(s)-\tilde{\sigma}(s)\right]+L \int_{t_{0}}^{t}\left\|\left(x_{n}^{\tilde{\sigma}_{n}}\right)_{s}-\left(x^{\tilde{\sigma}}\right)_{s}\right\|_{\infty} d \tilde{\sigma}(s)
\end{aligned}
$$

where we used (B) and (C) to obtain the last inequality. Thus, we get

$$
\left|x_{n}^{\tilde{\sigma}_{n}}(t)-x^{\tilde{\sigma}}(t)\right| \leq \varepsilon+2 \varepsilon M+L \int_{t_{0}}^{t}\left\|\left(x_{n}^{\tilde{\sigma}_{n}}\right)_{s}-\left(x^{\tilde{\sigma}}\right)_{s}\right\|_{\infty} \mathrm{d} \tilde{\sigma}(s)
$$

Using $\left(x_{n}^{\tilde{\sigma}_{n}}\right)_{t_{0}}=\phi^{\tilde{\sigma}_{n}}$ and $\left(x^{\tilde{\sigma}}\right)_{t_{0}}=\phi^{\tilde{\sigma}}$ and the uniform convergence $\phi^{\tilde{\sigma}_{n}} \rightarrow \phi^{\tilde{\sigma}}$, we have, for $n>N_{2}$,

$$
\left\|\left(x_{n}^{\tilde{\sigma}_{n}}\right)_{s}-x_{s}^{\tilde{\sigma}}\right\|_{\infty}=\sup _{\theta \in[-r, 0]}\left|x_{n}^{\tilde{\sigma}_{n}}(s+\theta)-x^{\tilde{\sigma}}(s+\theta)\right| \leq \varepsilon+\sup _{\eta \in[0, s]}\left|x_{n}^{\tilde{\sigma}_{n}}(\eta)-x^{\tilde{\sigma}}(\eta)\right|
$$

and, therefore,

$$
\left|x_{n}^{\tilde{\sigma}_{n}}(t)-x^{\tilde{\sigma}}(t)\right| \leq \varepsilon+2 \varepsilon M+L \int_{t_{0}}^{t}\left(\varepsilon+\sup _{\eta \in[0, s]}\left|x_{n}^{\tilde{\sigma}_{n}}(\eta)-x^{\tilde{\sigma}}(\eta)\right|\right) \mathrm{d} \tilde{\sigma}(s) .
$$

Then,

$$
\left|x_{n}^{\tilde{\sigma}_{n}}(t)-x^{\tilde{\sigma}}(t)\right| \leq \varepsilon+2 \varepsilon M+L \varepsilon\left(\tilde{\sigma}(t)-\tilde{\sigma}\left(t_{0}\right)\right)+\int_{t_{0}}^{t} L \sup _{\eta \in[0, s]}\left|x_{n}^{\tilde{\sigma}_{n}}(\eta)-x^{\tilde{\sigma}}(\eta)\right| \mathrm{d} \tilde{\sigma}(s) .
$$

Finally, by the Gronwall inequality (Theorem 2.10), we get

$$
\left|x_{n}^{\tilde{\sigma}_{n}}(t)-x^{\tilde{\sigma}}(t)\right| \leq \varepsilon\left(1+2 M+L\left(\tilde{\sigma}(t)-\tilde{\sigma}\left(t_{0}\right)\right)\right) e^{L\left(\tilde{\sigma}(t)-\tilde{\sigma}\left(t_{0}\right)\right)}
$$

and, since $\varepsilon>0$ is arbitrary, we have the desired result. 
Note that the hypothesis in Theorem 6.10 which guarantees that the sequence of functions $\left\{\tilde{\sigma}_{n}\right\}_{n=1}^{\infty}$ converges uniformly to $\tilde{\sigma}$ as $n \rightarrow \infty$ is necessary, since one cannot expect this to happen only using the fact that $d\left(\mathbb{T}_{n}, \mathbb{T}\right) \rightarrow 0$ as $n \rightarrow \infty$. Below, we present an example that illustrates this fact.

Example 6.11. Let $\mathbb{T}=[0, a] \cup[a+1, b]$ and $\mathbb{T}_{n}=[0, a+1 / n] \cup[a+1, b]$, for every $n \in \mathbb{N}$. Then $d\left(\mathbb{T}, \mathbb{T}_{n}\right)=1 / n \rightarrow 0$ as $n \rightarrow \infty$. However $\tilde{\sigma}_{n}(a+1 / n)=a+1 / n$, for every $n \in \mathbb{N}$, while $\tilde{\sigma}(a+1 / n)=a+1$. In other words, for every $n \geq 2$, there exists $t$ such that $\tilde{\sigma}(t)-\tilde{\sigma}_{n}(t) \geq 1 / 2$, which means that the sequence $\left\{\tilde{\sigma}_{n}\right\}_{n=1}^{\infty}$ does not converge uniformly to $\tilde{\sigma}$.

Even if we consider the Fell topology instead of the Hausdorff topology, the hypothesis of Theorem 6.10 guaranteeing the uniform convergence of the sequence of functions $\left\{\tilde{\sigma}_{n}\right\}_{n=1}^{\infty}$ is necessary. The next example illustrates this fact. Here, the notation $\operatorname{CL}(M)$ represents the set of all closed, nonempty subsets of $M$.

Example 6.12. Assume $\mathbb{R}$ with the usual metric and $\mathrm{CL}(\mathbb{R})$ is endowed with the Fell topology. Then, by [31, Lemma 4], we have

$$
\mathbb{T}_{n}=\{z+1 / n: z \in \mathbb{Z}\} \rightarrow \mathbb{Z}
$$

as $n \rightarrow \infty$. Also,

$$
\tilde{\sigma}_{n}(z+1 / n)=z+1 / n
$$

whereas $\tilde{\sigma}(z+1 / n)=z+1$, which implies that $\tilde{\sigma}_{n}$ does not converge uniformly to $\tilde{\sigma}$.

Notice that if we suppose that $\left\{\tilde{\sigma}_{n}\right\}_{n=1}^{\infty}$ converges uniformly to $\tilde{\sigma}$ as $n \rightarrow \infty$, we can suppress the hypothesis $d\left(\mathbb{T}_{n}, \mathbb{T}\right) \rightarrow 0$ as $n \rightarrow \infty$ to obtain our main result. We point out that the uniform convergence of $\left\{\tilde{\sigma}_{n}\right\}_{n=1}^{\infty}$ is enough to ensure the convergence of solutions of impulsive functional dynamic equations on time scales for variable time scales as we will prove later.

The next theorem shows that, under certain conditions, it is possible to obtain a correspondence between the solutions of impulsive measure functional differential equations, depending on the conditions about the functions $\tilde{\sigma}_{n}$ and $\tilde{\sigma}$. This result is new and it can be found in [16].

Theorem 6.13. Suppose $f: P \times\left[t_{0}, t_{0}+\eta\right] \rightarrow \mathbb{R}$ satisfies the conditions (A), (B) and $(\mathrm{C})$, and for each $k=1,2, \ldots, m$, the impulse operators $I_{k}: \mathbb{R}^{n} \rightarrow \mathbb{R}^{n}$ satisfy conditions 
$\left(\mathrm{A}^{*}\right)$ There exists a constant $K_{1}>0$ such that

$$
\left|I_{k}(x)\right| \leq K_{1}
$$

for every $k=1, \ldots, m$ and $x \in B$.

(B*) There exists a constant $K_{2}>0$ such that

$$
\left|I_{k}(x)-I_{k}(y)\right| \leq K_{2}|x-y|
$$

for every $k=1, \ldots, m$ and $x, y \in B$.

Moreover, suppose $x_{n}^{\tilde{\sigma}_{n}}$ is a solution of the system

$$
\left\{\begin{array}{l}
x_{n}^{\tilde{\sigma}_{n}}(t)=x_{n}^{\tilde{\sigma}_{n}}\left(t_{0}\right)+\int_{t_{0}}^{t} f\left(\left(x_{n}^{\tilde{\sigma}_{n}}\right)_{s}, s\right) \mathrm{d} \tilde{\sigma}_{n}(s)+\sum_{\substack{k \in\{1, \ldots, m\} \\
t_{k}<t}} I_{k}\left(x_{n}^{\tilde{\sigma}_{n}}\left(t_{k}\right)\right), \quad t \in \mathbb{T}_{n}^{*}, \\
\left(x_{n}^{\tilde{\sigma}_{n}}\right)_{t_{0}}=\phi^{\tilde{\sigma}_{n}}
\end{array}\right.
$$

and $x^{\tilde{\sigma}}$ is a solution of the measure functional differential equation given by

$$
\left\{\begin{array}{l}
x^{\tilde{\sigma}}(t)=x^{\tilde{\sigma}}\left(t_{0}\right)+\int_{t_{0}}^{t} f\left(x_{s}^{\tilde{\sigma}}, s\right) \mathrm{d} \tilde{\sigma}(s)+\sum_{\substack{k \in\{1, \ldots, m\} \\
t_{k}<t}} I_{k}\left(x^{\tilde{\sigma}}\left(t_{k}\right)\right), t \in \mathbb{T}^{*}, \\
x_{t_{0}}^{\tilde{\sigma}}=\phi^{\tilde{\sigma}}
\end{array}\right.
$$

Moreover, the sequence of functions $\left\{\tilde{\sigma}_{n}\right\}_{n=1}^{\infty}$ converges uniformly to $\tilde{\sigma}$ as $n \rightarrow \infty$. Also, suppose the sequence of initial conditions $\left\{\phi^{\tilde{\sigma}_{n}}\right\}_{n=1}^{\infty}$ converges uniformly to $\phi^{\tilde{\sigma}}$ as $n \rightarrow \infty$. Then, for every $\varepsilon>0$, there exists $N>0$ sufficiently large such that, for $n>N$, we have

$$
\left|x_{n}^{\tilde{\sigma}_{n}}(t)-x^{\tilde{\sigma}}(t)\right|<\varepsilon \text { for } t \in \mathbb{T}_{n}^{*} \cap \mathbb{T}^{*}
$$

Proof. Define the following function

$$
\tilde{f}(y, t)= \begin{cases}f(y, t), & t \in\left[t_{0}, t_{0}+\sigma\right] \backslash\left\{t_{1}, \ldots, t_{m}\right\} \\ I_{k}(y(0)), & t=t_{k} \text { for some } k \in\{1, \ldots, m\}\end{cases}
$$

Moreover, let $c_{1}, \ldots, c_{m} \in \mathbb{R}$ be constants such that the function $\overline{\tilde{\sigma}}:\left[t_{0}, t_{0}+\sigma\right] \rightarrow \mathbb{R}$ given 
by

$$
\overline{\tilde{\sigma}}(t)= \begin{cases}\tilde{\sigma}(t), & t \in\left[t_{0}, t_{1}\right], \\ \tilde{\sigma}(t)+c_{k}, & t \in\left(t_{k}, t_{k+1}\right], \text { for some } k \in\{1, \ldots, m-1\}, \\ \tilde{\sigma}(t)+c_{m}, & t \in\left(t_{m}, t_{0}+\sigma\right] .\end{cases}
$$

And also, define the following function $\overline{\tilde{\sigma}}_{n}:\left[t_{0}, t_{0}+\sigma\right] \rightarrow \mathbb{R}$ given by

$$
\overline{\tilde{\sigma}}_{n}(t)= \begin{cases}\tilde{\sigma}_{n}(t), & t \in\left[t_{0}, t_{1}\right], \\ \tilde{\sigma}_{n}(t)+c_{k}, & t \in\left(t_{k}, t_{k+1}\right], \text { for some } k \in\{1, \ldots, m-1\}, \\ \tilde{\sigma}_{n}(t)+c_{m}, & t \in\left(t_{m}, t_{0}+\sigma\right] .\end{cases}
$$

Since the sequence of functions $\left\{\tilde{\sigma}_{n}\right\}_{n=1}^{\infty}$ converges uniformly to $\tilde{\sigma}$, it follows immediately from the definition that the sequence of functions $\left\{\overline{\tilde{\sigma}}_{n}\right\}_{n=1}^{\infty}$ converges uniformly to $\overline{\tilde{\sigma}}$. Also, by Lemma 4.18, we obtain that all hypotheses of Theorem 6.10 are satisfied and then, using the correspondence (Theorem 4.23), the desired result follows.

Now, consider the following conditions concerning the function $f: G([-r, 0], B) \times$ $\left[t_{0}, t_{0}+\eta\right]_{\mathbb{T}_{n}} \rightarrow \mathbb{R}^{n}$

$\left(\mathrm{A}_{1}\right)$ The integral $\int_{t_{0}}^{t_{0}+\eta} f\left(y_{t}, t\right) \Delta t$ exists for every $y \in O$.

$\left(\mathrm{B}_{1}\right)$ There exists a constant $M>0$ such that

$$
\left|\int_{u_{1}}^{u_{2}} f\left(y_{t}, t\right) \Delta t\right| \leq M\left(u_{2}-u_{1}\right)
$$

for every $y \in O$ and $u_{1}, u_{2} \in\left[t_{0}, t_{0}+\eta\right]_{\mathbb{T}}, u_{1} \leq u_{2}$.

$\left(\mathrm{C}_{1}\right)$ There exists a constant $L>0$ such that

$$
\left|\int_{u_{1}}^{u_{2}}\left[f\left(y_{t}, t\right)-f\left(z_{t}, t\right)\right] \Delta t\right| \leq L \int_{u_{1}}^{u_{2}}\left\|y_{t}-z_{t}\right\|_{\infty} \Delta t
$$

for every $y, z \in O$ and $u_{1}, u_{2} \in\left[t_{0}, t_{0}+\eta\right]_{\mathbb{T}}, u_{1} \leq u_{2}$.

The next theorem is the main result of this section. It concerns continuous dependence of solutions on parameters for impulsive functional dynamic equations on time scales involving variable time scales. It is also a new result which can be found in [16]. 
Theorem 6.14. Suppose $x_{n}:\left[t_{0}-r, t_{0}+\eta\right]_{\mathbb{T}_{n}} \rightarrow \mathbb{R}^{n}$ is a solution of the impulsive functional dynamic equation on time scales

$$
\left\{\begin{array}{l}
x_{n}(t)=x_{n}\left(t_{0}\right)+\int_{t_{0}}^{t} f_{n}\left(\left(x_{n}^{\tilde{\sigma}_{n}}\right)_{s}, s\right) \Delta s+\sum_{\substack{k \in\{1, \ldots, m\} \\
t_{k}<t}} I_{k}\left(x_{n}\left(t_{k}\right)\right), \quad t \in\left[t_{0}, t_{0}+\eta\right]_{\mathbb{T}_{n}}, \\
x_{n}(t)=\phi(t), \quad t \in\left[t_{0}-r, t_{0}\right]_{\mathbb{T}_{n}},
\end{array}\right.
$$

where the functions $f_{n}: G([-r, 0], B) \times\left[t_{0}, t_{0}+\eta\right]_{\mathbb{T}_{n}} \rightarrow \mathbb{R}^{n}$ satisfy the conditions $\left(\mathrm{A}_{1}\right)$, $\left(\mathrm{B}_{1}\right)$ and $\left(\mathrm{C}_{1}\right)$. Also, suppose $x:\left[t_{0}-r, t_{0}+\eta\right]_{\mathbb{T}} \rightarrow \mathbb{R}^{n}$ is a solution of the impulsive functional dynamic equation on time scales

$$
\left\{\begin{array}{l}
x(t)=x\left(t_{0}\right)+\int_{t_{0}}^{t} f\left(x_{s}^{\tilde{\sigma}}, s\right) \Delta s+\sum_{\substack{k \in\{1, \ldots, m\} \\
t_{k}<t}} I_{k}\left(x\left(t_{k}\right)\right), \quad t \in\left[t_{0}, t_{0}+\eta\right]_{\mathbb{T}}, \\
x(t)=\phi(t), \quad t \in\left[t_{0}-r, t_{0}\right]_{\mathbb{T}},
\end{array}\right.
$$

where $f: G([-r, 0], B) \times\left[t_{0}, t_{0}+\eta\right]_{\mathbb{T}} \rightarrow \mathbb{R}^{n}$ satisfies conditions $\left(\mathrm{A}_{1}\right),\left(\mathrm{B}_{1}\right)$ and $\left(\mathrm{C}_{1}\right)$ and for each $k=1,2, \ldots, m$, the impulse operators $I_{k}: \mathbb{R}^{n} \rightarrow \mathbb{R}^{n}$ satisfy conditions $\left(\mathrm{A}^{*}\right)$ and $\left(\mathrm{B}^{*}\right)$. Suppose that the sequence of functions $\left\{\tilde{\sigma}_{n}\right\}_{n=1}^{\infty}$ converges uniformly to $\tilde{\sigma}$ as $n \rightarrow \infty$. Also, suppose the sequence $\left\{\phi^{\tilde{\sigma}_{n}}\right\}_{n=1}^{\infty}$ converges uniformly to $\phi^{\tilde{\sigma}}$ as $n \rightarrow \infty$. Then, for every $\varepsilon>0$, there exists $N>0$ sufficiently large such that, for $n>N$, we have

$$
\left|x_{n}(t)-x(t)\right|<\varepsilon \text { for } t \in \mathbb{T}_{n} \cap \mathbb{T} \text {. }
$$

Proof. Since the function $f_{n}: G([-r, 0], B) \times\left[t_{0}, t_{0}+\eta\right]_{\mathbb{T}} \rightarrow \mathbb{R}^{n}$ satisfies the conditions $\left(\mathrm{A}_{1}\right),\left(\mathrm{B}_{1}\right)$ and $\left(\mathrm{C}_{1}\right)$, it follows from Lemma 4.24 that the corresponding conditions $(\mathrm{A})$, (B) and (C) are satisfied for the extension of $f_{n}$ and, therefore, all hypotheses from Theorem 6.13 are satisfied, and the desired result follows immediately applying the correspondence between impulsive measure functional differential equations and impulsive functional dynamic equations on time scales. 



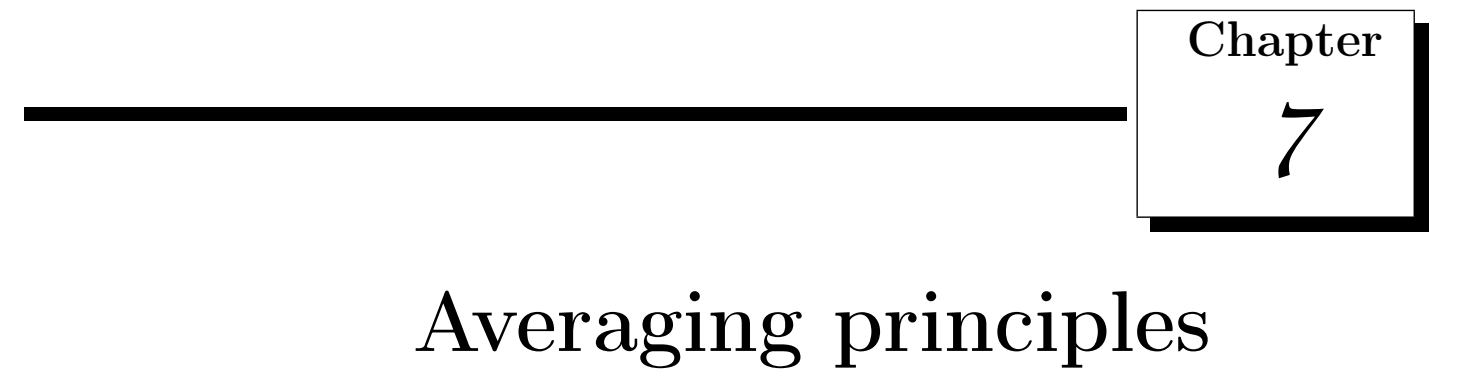

The right-hand side of the equations which model problems in celestial mechanic have terms which oscillate quickly and terms which oscillate slowly. The terms which oscillate slowly indicate the slow evolution of the system of parameters. On the other hand, the terms which oscillate quickly almost do not affect the motion and, thus, they can be omitted. The process of omitting the terms which oscillate quickly on the right-hand side of the equation is called an averaging.

The purpose of an averaging method or principle is to give conditions under which the solutions of a time varying system can be approximated by solutions of an autonomous differential system. The averaging method is, therefore, a powerful tool in studying the perturbation theory of differential equations, since it allows one to replace a time-varying small perturbation, acting on a long time interval, by a time-invariant perturbation and, in this process, only a small error is introduced.

This chapter is devoted to presenting averaging principles for various types of equations. In the first section, we investigate non-periodic averaging methods. In the second section, we investigate periodic averaging methods for various types of equations and finally, in the last section, we present some examples to illustrate the applications of averaging principles.

We emphasize that almost all the results presented here are new and they are contained in the papers [34] and [84]. 


\subsection{Non-periodic averaging principles}

Consider the ordinary differential equation

$$
\left\{\begin{array}{l}
\dot{x}=\varepsilon f(x(t), t)+\varepsilon^{2} g(x(t), t, \varepsilon), \\
x\left(t_{0}\right)=x_{0}
\end{array}\right.
$$

where $\varepsilon>0$ is a small parameter, $t_{0}>0, f: \mathbb{R}^{n} \times \mathbb{R} \rightarrow \mathbb{R}^{n}$ and $g: \mathbb{R}^{n} \times \mathbb{R}^{n} \times\left(0, \varepsilon_{0}\right) \rightarrow \mathbb{R}^{n}$. Consider, also, the autonomous averaged differential equation

$$
\left\{\begin{array}{l}
\dot{y}=\varepsilon f_{0}(y(t)) \\
y\left(t_{0}\right)=x_{0}
\end{array}\right.
$$

where

$$
f_{0}(y)=\lim _{T \rightarrow \infty} \frac{1}{T} \int_{t_{0}}^{t_{0}+T} f(y, t) d t
$$

A non-periodic averaging principle for (7.1.1) establishes conditions under which one can obtain an approximation between the solutions of problem (7.1.1) and the solutions of the averaged problem (7.1.2). Therefore, averaging principles are powerful tools in the study of perturbations of differential equations.

Coming back to historical details, we can say that the first justifications of non-periodic averaging principles for nonlinear system were presented by N. N. Bogolyubov and A. Mitropolskii [15] and by N. N. Krylov and N. N. Bogolyubov [14]. Their results are know as KBM averaging method.

Throughout the years, several authors published papers on averaging methods for different types of differential systems. In the 60's, authors as, for instance, V. I. Fodčuk [44], A. Halanay [53], J. K. Hale [54], G. N. Medvedev [82] and Medvedev et al. [83] developed methods of averaging for certain functional differential equations (we write FDEs for short), with a small parameter, approximating them by autonomous ordinary differential equations (ODEs).

It was only in the 70's that the investigations on averaging for FDEs indicated that the classic approximation of solutions by solutions of an autonomous ODE could be replaced by an approximation by solutions of an autonomous FDE. In this direction, we can mention the papers by V. Strygin [101] and by B. Lehman and S. P. Weibel [80]. See also [77], [78] and [79]. 
In the late 80's, D. D. Bainov and S. D. Milusheva [8] considered a FDE of neutral type with impulses, approximating it by autonomous ODEs.

Recently, in [74] and [75], the authors stated averaging results for FDEs employing the tools of non-standard analysis. Also, based in such papers, the authors of [35] improved the results from [74] and [75] using standard analysis though.

While the theory of averaging for functional differential equations is well developed, the literature involving averaging principles for measure functional differential equations is scarce. As a matter of fact, we do not know any result in this direction. Also, because the theory of functional dynamic equations on time scales is very recent (see [36]), the literature concerning averaging principles for these equations is scarce as well.

We divide this first section into two subsections. In the first subsection, we prove a non-periodic averaging principle for measure functional differential equations and, using the correspondence presented in Chapter 4, we prove a non-periodic averaging principle for functional dynamic equations on time scales. The second subsection brings nonperiodic averaging principles for impulsive measure functional differential equations and for impulsive functional dynamic equations on time scales.

\subsubsection{Measure FDEs and functional dynamic equations on time scales}

In this section, we focus our attention on an initial value problem for a measure functional differential equation of the form

$$
\left\{\begin{array}{l}
D x=\varepsilon f\left(x_{t}, t\right) D h_{1}(t)+\varepsilon^{2} g\left(x_{t}, t, \varepsilon\right) D h_{2}(t) \\
x_{0}=\phi
\end{array}\right.
$$

where $\varepsilon>0$ is a small parameter, $D x, D h_{1}$ and $D h_{2}$ denote, respectively, the distributional derivatives of the functions $x, h_{1}$ and $h_{2}$ in the sense of distributions of L. Schwartz. The integral form equivalent to (7.1.3) is given by

$$
\left\{\begin{array}{l}
x(t)=x_{0}+\varepsilon \int_{0}^{t} f\left(x_{s}, s\right) \mathrm{d} h_{1}(s)+\varepsilon^{2} \int_{0}^{t} g\left(x_{s}, s, \varepsilon\right) \mathrm{d} h_{2}(s), \\
x_{0}=\phi
\end{array}\right.
$$

where we consider that the initial function, $\phi$, is an element of $G^{-}\left([-r, 0], \mathbb{R}^{n}\right)$ and we consider the Kurzweil-Henstock-Stieljtes integral in (7.1.4) are taken with respect to non- 
decreasing functions $h_{1}$ and $h_{2}$, where $h_{1}, h_{2}:[0, \infty) \rightarrow \mathbb{R}$.

In order to obtain an averaging principle for (7.1.3), we consider the auxiliary initial value problem

$$
\left\{\begin{array}{l}
D x=\varepsilon f\left(x_{t}, \frac{t}{\varepsilon}\right) D h_{1}\left(\frac{t}{\varepsilon}\right)+\varepsilon^{2} g\left(x_{t}, \frac{t}{\varepsilon}, \varepsilon\right) D h_{2}\left(\frac{t}{\varepsilon}\right), \quad t \in[0, L] \\
x_{0}=\phi
\end{array}\right.
$$

where $\phi \in G^{-}\left([-r, 0], \mathbb{R}^{n}\right), L>0$ and there exists a small parameter $\varepsilon_{0}$ such that $\varepsilon \in$ $\left(0, \varepsilon_{0}\right]$. We assume that $f$ maps any pair $(\psi, t) \in G^{-}\left([-r, 0], \mathbb{R}^{n}\right) \times[0, \infty)$ into $\mathbb{R}^{n}$ and that the mapping $t \mapsto f\left(y_{t}, t\right)$ is Kurzweil-Henstock integrable with respect to $h_{1}$, for all $t \in$ $[0, \infty)$. Furthermore, we consider that $g$ maps $(\psi, t, \varepsilon) \in G^{-}\left([-r, 0], \mathbb{R}^{n}\right) \times[0, \infty) \times\left(0, \varepsilon_{0}\right]$ into $\mathbb{R}^{n}$ and that the mapping $t \mapsto g\left(y_{t}, t, \varepsilon\right)$ is Kurzweil-Henstock integrable with respect to $h_{2}$, for all $t \in[0, \infty)$.

Note that, by a change of variables, we can transform system (7.1.5) into system (7.1.3). Indeed, if $x$ is a solution of $(7.1 .5)$ on $[0, L]$, then

$$
x(t)=x(0)+\varepsilon \int_{0}^{t} f\left(x_{s}, \frac{s}{\varepsilon}\right) \mathrm{d} h_{1}\left(\frac{s}{\varepsilon}\right)+\varepsilon^{2} \int_{0}^{t} g\left(x_{s}, \frac{s}{\varepsilon}, \varepsilon\right) \mathrm{d} h_{2}\left(\frac{s}{\varepsilon}\right) .
$$

Thus, using the change of variables $s \mapsto s / \varepsilon$, we obtain

$$
x(t)=x(0)+\varepsilon \int_{0}^{t / \varepsilon} f\left(x_{\varepsilon \tau, \varepsilon}, \tau\right) \mathrm{d} h_{1}(\tau)+\varepsilon^{2} \int_{0}^{t / \varepsilon} g\left(x_{\varepsilon \tau, \varepsilon} \tau, \varepsilon\right) \mathrm{d} h_{2}(\tau),
$$

where $x_{t, \varepsilon}(\theta)=x(t+\varepsilon \theta), \theta \in[-r, 0]$. Then, if we put $y(t / \varepsilon)=x(t)$, we obtain, for $t \in[0, L / \varepsilon]$,

$$
\begin{aligned}
y(t)-y(0) & =x(\varepsilon t)-x(0) \\
& =\varepsilon \int_{0}^{\varepsilon t / \varepsilon} f\left(x_{\varepsilon s, \varepsilon}, s\right) \mathrm{d} h_{1}(s)+\varepsilon^{2} \int_{0}^{\varepsilon t / \varepsilon} g\left(x_{\varepsilon s, \varepsilon}, s, \varepsilon\right) \mathrm{d} h_{2}(s) \\
& =\varepsilon \int_{0}^{t} f\left(y_{s}, s\right) \mathrm{d} h_{1}(s)+\varepsilon^{2} \int_{0}^{t} g\left(y_{s}, s, \varepsilon\right) \mathrm{d} h_{2}(s),
\end{aligned}
$$

which means that $y$ is a solution of $(7.1 .3)$ on $\left[0, \frac{L}{\varepsilon}\right]$. Thus, if we have a solution of the measure functional differential equation $(7.1 .5)$ on $[0, L]$, then, by a change of variables, we can obtain a solution of (7.1.3) on $\left[0, \frac{L}{\varepsilon}\right]$ and vice-versa. We are then interested in establishing an averaging result for (7.1.5). Then, an averaging principle for (7.1.3) will follow naturally. 
Let $r, \sigma \in \mathbb{R}$, with $r, \sigma>0$, and let $O$ be an open subset of $G^{-}\left([-r, \sigma], \mathbb{R}^{n}\right)$.

We assume that $f: G^{-}\left([-r, 0], \mathbb{R}^{n}\right) \times[0, \infty) \rightarrow \mathbb{R}^{n}$ satisfies the following conditions:

$\left(H_{1}\right)$ For every $x \in O$ and every $t \in[0, \infty)$, the integral $\int_{0}^{t} f\left(x_{s}, s\right) \mathrm{d} h_{1}(s)$ exists;

$\left(H_{2}\right)$ There exists a constant $M>0$ such that, for every $y, z \in O$ and every $u_{1}, u_{2} \in$ $[0, \infty)$

$$
\left|\int_{u_{1}}^{u_{2}}\left[f\left(y_{s}, s\right)-f\left(z_{s}, s\right)\right] \mathrm{d} h_{1}(s)\right| \leq M \int_{u_{1}}^{u_{2}}\left\|y_{s}-z_{s}\right\| \mathrm{d} h_{1}(s) .
$$

Consider the following assumptions on $g: G^{-}\left([-r, 0], \mathbb{R}^{n}\right) \times[0, \infty) \times\left(0, \varepsilon_{0}\right] \rightarrow \mathbb{R}^{n}$ :

$\left(H_{3}\right)$ For every $x \in O, t \in[0, \infty)$ and $\varepsilon \in\left(0, \varepsilon_{0}\right]$, the integral $\int_{0}^{t} g\left(x_{s}, s, \varepsilon\right) \mathrm{d} h_{2}(s)$ exists.

$\left(H_{4}\right)$ There is a constant $C>0$ such that, for all $y \in O, \varepsilon \in\left(0, \varepsilon_{0}\right]$ and $u_{1}, u_{2} \in[0, \infty)$,

$$
\left|\int_{u_{1}}^{u_{2}} g\left(y_{s}, s, \varepsilon\right) \mathrm{d} h_{2}(s)\right| \leq C \int_{u_{1}}^{u_{2}} \mathrm{~d} h_{2}(s) .
$$

Consider $h_{1}:[0, \infty) \rightarrow \mathbb{R}$ being a nondecreasing function which satisfies:

$\left(H_{5}\right)$ There exists a constant $K>0$ such that, for every $\beta \geq 0$,

$$
\limsup _{T \rightarrow \infty} \frac{h_{1}(T+\beta)-h_{1}(\beta)}{T} \leq K .
$$

Moreover, assume that $h_{2}:[0, \infty) \rightarrow \mathbb{R}$ is a nondecreasing function such that:

$\left(H_{6}\right)$ There exists a constant $N>0$ such that, for every $\beta \geq 0$,

$$
\limsup _{T \rightarrow \infty} \frac{h_{2}(T+\beta)-h_{2}(\beta)}{T} \leq N
$$

Suppose, for each $\psi \in G^{-}\left([-r, 0], \mathbb{R}^{n}\right)$, the limit

$$
f_{0}(\psi)=\lim _{T \rightarrow \infty} \frac{1}{T} \int_{0}^{T} f(\psi, s) \mathrm{d} h_{1}(s)
$$

exists, where the integral has to be understood in the sense of Kurzweil-Henstock-Stieltjes, and consider the averaged functional differential equation

$$
\left\{\begin{array}{l}
\dot{y}=f_{0}\left(y_{t}\right), \quad t \in[0, L / \varepsilon] \\
y_{0}=\phi
\end{array}\right.
$$


where $f_{0}$ is given by (7.1.6). If the conditions $\left(H_{2}\right)$ and $\left(H_{5}\right)$ are satisfied, then

$$
\begin{aligned}
\left|f_{0}(\phi)-f_{0}(\psi)\right| & =\left|\lim _{T \rightarrow \infty} \frac{1}{T} \int_{0}^{T} f(\phi, s) \mathrm{d} h_{1}(s)-\lim _{T \rightarrow \infty} \frac{1}{T} \int_{0}^{T} f(\psi, s) \mathrm{d} h_{1}(s)\right| \\
& \leq \lim _{T \rightarrow \infty} \frac{1}{T} \int_{0}^{T} M\|\phi-\psi\|_{\infty} \mathrm{d} h_{1}(s) \\
& \leq M\|\phi-\psi\|_{\infty} \limsup _{T \rightarrow \infty} \frac{h_{1}(T)-h_{1}(0)}{T} \leq M K\|\phi-\psi\|_{\infty} .
\end{aligned}
$$

In particular, for every $y \in O$ and every $t, s \in[0, \infty)$, we have

$$
\left|f_{0}\left(y_{s}\right)-f_{0}\left(y_{t}\right)\right| \leq M K\left\|y_{s}-y_{t}\right\|_{\infty}
$$

which implies that, if $y \in O$ is a solution of (7.1.7) and $s, t \in[0, \infty)$, with $t \leq s$, then, for $\theta \in[-r, 0]$, we have

$$
\begin{aligned}
|y(s+\theta)-y(t+\theta)| & =\left|\int_{t+\theta}^{s+\theta} f_{0}\left(y_{\sigma}\right) d \sigma\right| \\
& \leq \int_{t+\theta}^{s+\theta}\left|f_{0}\left(y_{\sigma}\right)-f_{0}(0)\right| d \sigma+\int_{t+\theta}^{s+\theta}\left|f_{0}(0)\right| d \sigma \\
& \leq M K \int_{t+\theta}^{s+\theta} \sup _{\sigma \in[t-r, s]}\left\|y_{\sigma}\right\|_{\infty} d \sigma+(s-t)\left|f_{0}(0)\right|
\end{aligned}
$$

and, hence,

$$
\begin{aligned}
\left\|y_{s}-y_{t}\right\|_{\infty} & =\sup _{\theta \in[-r, 0]}|y(s+\theta)-y(t+\theta)| \\
& \leq M K(s-t) \sup _{\sigma \in[t-r, s]}\left\|y_{\sigma}\right\|_{\infty}+(s-t)\left|f_{0}(0)\right|
\end{aligned}
$$

Thus, as $s-t \rightarrow 0^{+},\left\|y_{s}-y_{t}\right\|_{\infty} \rightarrow 0$, that is, $y_{t}$ as a function of $t$, with $t \in[0, \infty)$, is a continuous function.

Now, we present an original auxiliary lemma which will be essential to our purposes. Its proof is inspired in the proof of Lemma 3.1 in [35].

Lemma 7.1. For every $t>0$ and every $\alpha>0$, we have

$$
\lim _{\varepsilon \rightarrow 0+} \frac{1}{\alpha / \varepsilon} \int_{t / \varepsilon}^{t / \varepsilon+\alpha / \varepsilon} f(\psi, s) \mathrm{d} h_{1}(s)=f_{0}(\psi), \quad \psi \in G^{-}\left([-r, 0], \mathbb{R}^{n}\right),
$$

where $f_{0}$ is given by (7.1.6). 
Proof. By definition,

$$
\lim _{T \rightarrow \infty} \frac{1}{T} \int_{0}^{T} f(\psi, s) \mathrm{d} h_{1}(s)=f_{0}(\psi), \quad \psi \in G^{-}\left([-r, 0], \mathbb{R}^{n}\right)
$$

Thus, clearly, for $t>0$ and $\alpha>0$, we also have

$$
\lim _{\varepsilon \rightarrow 0^{+}} \frac{1}{t / \varepsilon+\alpha / \varepsilon} \int_{0}^{t / \varepsilon+\alpha / \varepsilon} f(\psi, s) \mathrm{d} h_{1}(s)=f_{0}(\psi)
$$

and

$$
\lim _{\varepsilon \rightarrow 0^{+}} \frac{1}{t / \varepsilon} \int_{0}^{t / \varepsilon} f(\psi, s) \mathrm{d} h_{1}(s)=f_{0}(\psi) .
$$

Then,

$$
\begin{gathered}
\lim _{\varepsilon \rightarrow 0^{+}}\left[\frac{1}{t / \varepsilon+\alpha / \varepsilon} \int_{0}^{t / \varepsilon+\alpha / \varepsilon} f(\psi, s) \mathrm{d} h_{1}(s)-\frac{1}{t / \varepsilon} \int_{0}^{t / \varepsilon} f(\psi, s) \mathrm{d} h_{1}(s)\right] \\
=\lim _{\varepsilon \rightarrow 0^{+}}\left[\frac{1}{t / \varepsilon+\alpha / \varepsilon} \int_{0}^{t / \varepsilon+\alpha / \varepsilon} f(\psi, s) \mathrm{d} h_{1}(s)-f_{0}(\psi)\right] \\
+\lim _{\varepsilon \rightarrow 0^{+}}\left[f_{0}(\psi)-\frac{1}{t / \varepsilon} \int_{0}^{t / \varepsilon} f(\psi, s) \mathrm{d} h_{1}(s)\right]=0 .
\end{gathered}
$$

On the other hand,

$$
\begin{gathered}
\frac{\varepsilon}{\alpha} \int_{t / \varepsilon}^{t / \varepsilon+\alpha / \varepsilon} f(\psi, s) \mathrm{d} h_{1}(s)=\frac{1}{\alpha / \varepsilon} \int_{0}^{t / \varepsilon+\alpha / \varepsilon} f(\psi, s) \mathrm{d} h_{1}(s)-\frac{1}{\alpha / \varepsilon} \int_{0}^{t / \varepsilon} f(\psi, s) \mathrm{d} h_{1}(s) \\
=\left(\frac{t / \varepsilon+\alpha / \varepsilon}{t / \varepsilon+\alpha / \varepsilon}\right) \frac{1}{\alpha / \varepsilon} \int_{0}^{t / \varepsilon+\alpha / \varepsilon} f(\psi, s) \mathrm{d} h_{1}(s)-\left(\frac{t / \varepsilon}{t / \varepsilon}\right) \frac{1}{\alpha / \varepsilon} \int_{0}^{t / \varepsilon} f(\psi, s) \mathrm{d} h_{1}(s) \\
=\frac{1}{t / \varepsilon+\alpha / \varepsilon}\left(\frac{t}{\alpha}+1\right) \int_{0}^{t / \varepsilon+\alpha / \varepsilon} f(\psi, s) \mathrm{d} h_{1}(s)-\frac{t}{\alpha} \cdot \frac{1}{t / \varepsilon} \int_{0}^{t / \varepsilon} f(\psi, s) \mathrm{d} h_{1}(s) \\
=\frac{1}{t / \varepsilon+\alpha / \varepsilon} \int_{0}^{t / \varepsilon+\alpha / \varepsilon} f(\psi, s) \mathrm{d} h_{1}(s)+ \\
+\frac{t}{\alpha}\left[\frac{1}{t / \varepsilon+\alpha / \varepsilon} \int_{0}^{t / \varepsilon+\alpha / \varepsilon} f(\psi, s) \mathrm{d} h_{1}(s)-\frac{1}{t / \varepsilon} \int_{0}^{t / \varepsilon} f(\psi, s) \mathrm{d} h_{1}(s)\right]
\end{gathered}
$$

Therefore,

$$
\lim _{\varepsilon \rightarrow 0^{+}}\left[\frac{\varepsilon}{\alpha} \int_{t / \varepsilon}^{t / \varepsilon+\alpha / \varepsilon} f(\psi, s) \mathrm{d} h_{1}(s)-f_{0}(\psi)\right]
$$




$$
\begin{gathered}
=\lim _{\varepsilon \rightarrow 0^{+}}\left[\frac{1}{t / \varepsilon+\alpha / \varepsilon} \int_{0}^{t / \varepsilon+\alpha / \varepsilon} f(\psi, s) \mathrm{d} h_{1}(s)-f_{0}(\psi)\right] \\
+\lim _{\varepsilon \rightarrow 0^{+}} \frac{t}{\alpha}\left[\frac{1}{t / \varepsilon+\alpha / \varepsilon} \int_{0}^{t / \varepsilon+\alpha / \varepsilon} f(\psi, s) \mathrm{d} h_{1}(s)-\frac{1}{t / \varepsilon} \int_{0}^{t / \varepsilon} f(\psi, s) \mathrm{d} h_{1}(s)\right]=0 .
\end{gathered}
$$

Hence

$$
\lim _{\varepsilon \rightarrow 0^{+}} \frac{\varepsilon}{\alpha} \int_{t / \varepsilon}^{t / \varepsilon+\alpha / \varepsilon} f(\psi, s) \mathrm{d} h_{1}(s)=f_{0}(\psi)
$$

and the result follows.

The next corollary follows easily from Lemma 7.1.

Corollary 7.2. Let $t>0$ and $\alpha>0$. Then, for every $y \in O$, we have

$$
\lim _{\varepsilon \rightarrow 0+} \frac{\varepsilon}{\alpha} \int_{t / \varepsilon}^{t / \varepsilon+\alpha / \varepsilon} f\left(y_{t}, s\right) \mathrm{d} h_{1}(s)=f_{0}\left(y_{t}\right)
$$

The proof of the next lemma is inspired in [35], Lemma 3.2. Such result is new and it can be found in [34].

Lemma 7.3. Suppose $[0, b)$ is the maximal interval of existence of solution of (7.1.7), where $b$ is finite, and $y$ is a maximal solution of (7.1.7). Moreover, suppose $f$ satisfies conditions $\left(H_{1}\right)$ and $\left(H_{2}\right)$ and $h_{1}$ satisfies condition $\left(H_{5}\right)$. Then, given $\varepsilon>0$, there exists $\mu>0$ such that

$$
\left|\varepsilon \int_{0}^{t} f\left(y_{s}, \frac{s}{\varepsilon}\right) \mathrm{d} h_{1}\left(\frac{s}{\varepsilon}\right)-\int_{0}^{t} f_{0}\left(y_{s}\right) d s\right|<\mu, \quad t \in[0, b),
$$

and $\mu$ tends to zero when $\varepsilon \rightarrow 0^{+}$.

Proof. For $\varepsilon>0$ and $\psi \in G^{-}\left([-r, \infty), \mathbb{R}^{n}\right)$, define

$$
\int_{0}^{t} f_{1}\left(\psi, \frac{s}{\varepsilon}\right) \mathrm{d} h_{1}\left(\frac{s}{\varepsilon}\right)=\varepsilon \int_{0}^{t} f\left(\psi, \frac{s}{\varepsilon}\right) \mathrm{d} h_{1}\left(\frac{s}{\varepsilon}\right)-\int_{0}^{t} f_{0}(\psi) d s, \quad t \geq 0 .
$$

Let $\delta$ be a gauge corresponding to $\varepsilon>0$ in the definition of the Kurzweil-HenstockStieltjes integral

$$
\int_{0}^{t} f\left(y_{\sigma}, \frac{\sigma}{\varepsilon}\right) \mathrm{d} h_{1}\left(\frac{\sigma}{\varepsilon}\right)=\int_{0}^{t / \varepsilon} f\left(y_{s \varepsilon, \varepsilon}, s\right) \mathrm{d} h_{1}(s)
$$


and consider a $\delta$-fine tagged partition $\left\{\left(\tau_{i},\left[s_{i}, s_{i+1}\right]\right), i=0,1,2, \ldots, m-1\right\}$ of $[0, t]$. Then,

$$
\begin{gathered}
\left|\varepsilon \int_{0}^{t} f\left(y_{s}, \frac{s}{\varepsilon}\right) \mathrm{d} h_{1}\left(\frac{s}{\varepsilon}\right)-\int_{0}^{t} f_{0}\left(y_{s}\right) d s\right|=\left|\int_{0}^{t} f_{1}\left(y_{s}, \frac{s}{\varepsilon}\right) \mathrm{d} h_{1}\left(\frac{s}{\varepsilon}\right)\right| \\
\leq \sum_{i=0}^{m-1}\left|\int_{s_{i}}^{s_{i+1}}\left[f_{1}\left(y_{s}, \frac{s}{\varepsilon}\right)-f_{1}\left(y_{s_{i}}, \frac{s}{\varepsilon}\right)\right] \mathrm{d} h_{1}\left(\frac{s}{\varepsilon}\right)\right|+\sum_{i=0}^{m-1}\left|\int_{s_{i}}^{s_{i+1}} f_{1}\left(y_{s_{i}}, \frac{s}{\varepsilon}\right) \mathrm{d} h_{1}\left(\frac{s}{\varepsilon}\right)\right| \\
\leq \sum_{i=0}^{m-1}\left|\varepsilon \int_{s_{i}}^{s_{i+1}}\left[f\left(y_{s}, \frac{s}{\varepsilon}\right)-f\left(y_{s_{i}}, \frac{s}{\varepsilon}\right)\right] \mathrm{d} h_{1}\left(\frac{s}{\varepsilon}\right)\right|+\sum_{i=0}^{m-1}\left|\int_{s_{i}}^{s_{i+1}}\left[f_{0}\left(y_{s}\right)-f_{0}\left(y_{s_{i}}\right)\right] d s\right| \\
+\sum_{i=0}^{m-1}\left|\int_{s_{i}}^{s_{i+1}} f_{1}\left(y_{s_{i}}, \frac{s}{\varepsilon}\right) \mathrm{d} h_{1}\left(\frac{s}{\varepsilon}\right)\right| .
\end{gathered}
$$

We can suppose, without loss of generality, that the gauge $\delta$ is such that $\delta(\xi)<\frac{\varepsilon}{2}$, for every $\xi \in[0, t]$. Then, by (7.1.9), for each $i=0,1,2, \ldots, m-1$, and each $s \in\left[s_{i}, s_{i+1}\right]$, we have

$$
\begin{aligned}
\left\|y_{s}-y_{s_{i}}\right\|_{\infty} & <M K\left(s-s_{i}\right) \sup _{\sigma \in\left[s_{i}-r, s\right]}\left\|y_{\sigma}\right\|_{\infty}+\left(s-s_{i}\right)\left|f_{0}(0)\right| \\
& <M K 2 \delta\left(\tau_{i}\right) \sup _{\sigma \in\left[s_{i}-r, s_{i+1}\right]}\left\|y_{\sigma}\right\|_{\infty}+2 \delta\left(\tau_{i}\right)\left|f_{0}(0)\right| \\
& <M K \varepsilon \sup _{\sigma \in\left[s_{i}-r, s_{i+1}\right]}\left\|y_{\sigma}\right\|_{\infty}+\varepsilon\left|f_{0}(0)\right| .
\end{aligned}
$$

Therefore, for each $i=0,1,2, \ldots, m-1$,

$$
\sup _{s \in\left[s_{i}, s_{i+1}\right]}\left\|y_{s}-y_{s_{i}}\right\|_{\infty} \leq \varepsilon\left(M K \sup _{\sigma \in[-r, b)}\left\|y_{\sigma}\right\|_{\infty}+\left|f_{0}(0)\right|\right) .
$$

Then, taking

$$
D=M K \sup _{\sigma \in[-r, b)}\left\|y_{\sigma}\right\|_{\infty}+\left|f_{0}(0)\right|
$$

we obtain

$$
\sup _{s \in\left[s_{i}, s_{i+1}\right]}\left\|y_{s}-y_{s_{i}}\right\|_{\infty} \leq \varepsilon D, \quad i=0,1,2, \ldots, m-1 .
$$

Now, (7.1.12) and conditions $\left(H_{2}\right)$ and $\left(H_{5}\right)$ imply

$$
\begin{gathered}
\sum_{i=0}^{m-1}\left|\int_{s_{i}}^{s_{i+1}} \varepsilon\left[f\left(y_{s}, \frac{s}{\varepsilon}\right)-f\left(y_{s_{i}}, \frac{s}{\varepsilon}\right)\right] \mathrm{d} h_{1}\left(\frac{s}{\varepsilon}\right)\right| \\
\leq \varepsilon M \sum_{i=0}^{m-1} \sup _{\sigma \in\left[s_{i}, s_{i+1}\right]}\left\|y_{\sigma}-y_{s_{i}}\right\|_{\infty} \int_{s_{i}}^{s_{i+1}} \mathrm{~d} h_{1}\left(\frac{s}{\varepsilon}\right) \leq \varepsilon^{2} D M \sum_{i=0}^{m-1}\left[h_{1}\left(\frac{s_{i+1}}{\varepsilon}\right)-h_{1}\left(\frac{s_{i}}{\varepsilon}\right)\right]
\end{gathered}
$$




$$
\begin{gathered}
=\varepsilon D M \sum_{i=0}^{m-1} \frac{\varepsilon}{s_{i+1}-s_{i}}\left(s_{i+1}-s_{i}\right)\left[h_{1}\left(\frac{s_{i+1}-s_{i}}{\varepsilon}+\frac{s_{i}}{\varepsilon}\right)-h_{1}\left(\frac{s_{i}}{\varepsilon}\right)\right] \\
\leq \varepsilon D M \sum_{i=0}^{m} K\left(s_{i+1}-s_{i}\right)=\varepsilon D M K t<\varepsilon D M K b .
\end{gathered}
$$

Then, using (7.1.8) and (7.1.12), for each $i=0,1,2, \ldots, m-1$ and each $s \in\left[s_{i}, s_{i+1}\right]$, we have

$$
\begin{gathered}
\sum_{i=0}^{m-1}\left|\int_{s_{i}}^{s_{i+1}}\left[f_{0}\left(y_{s}\right)-f_{0}\left(y_{s_{i}}\right)\right] d s\right| \leq M K \sum_{i=0}^{m-1} \sup _{\sigma \in\left[s_{i}, s_{i+1}\right]}\left\|y_{\sigma}-y_{s_{i}}\right\|_{\infty}\left(s_{i+1}-s_{i}\right) \\
\leq \varepsilon D M K \sum_{i=0}^{m-1}\left(s_{i+1}-s_{i}\right)=\varepsilon D M K t<\varepsilon D M K b .
\end{gathered}
$$

Finally, we assert that the sum

$$
\sum_{i=0}^{m-1}\left|\int_{s_{i}}^{s_{i+1}} f_{1}\left(y_{s_{i}}, \frac{s}{\varepsilon}\right) \mathrm{d} h_{1}\left(\frac{s}{\varepsilon}\right)\right|
$$

can be made arbitrarily small by Corollary 7.2. Indeed, for each $\alpha_{i}=s_{i+1}-s_{i}, i=$ $0,1,2, \ldots, m-1$, we have

$$
\begin{gathered}
\sum_{i=0}^{m-1}\left|\int_{s_{i}}^{s_{i+1}} f_{1}\left(y_{s_{i}}, \frac{s}{\varepsilon}\right) \mathrm{d} h_{1}\left(\frac{s}{\varepsilon}\right)\right|=\sum_{i=0}^{m-1}\left|\varepsilon \int_{s_{i}}^{s_{i+1}} f\left(y_{s_{i}}, \frac{s}{\varepsilon}\right) \mathrm{d} h_{1}\left(\frac{s}{\varepsilon}\right)-\int_{s_{i}}^{s_{i+1}} f_{0}\left(y_{s_{i}}\right) d s\right| \\
=\sum_{i=0}^{m-1}\left|\varepsilon \int_{s_{i}}^{s_{i}+\alpha_{i}} f\left(y_{s_{i}}, \frac{s}{\varepsilon}\right) \mathrm{d} h_{1}\left(\frac{s}{\varepsilon}\right)-\int_{s_{i}}^{s_{i}+\alpha_{i}} f_{0}\left(y_{s_{i}}\right) d s\right| \\
=\sum_{i=0}^{m-1} \alpha_{i}\left|\frac{\varepsilon}{\alpha_{i}} \int_{s_{i} / \varepsilon}^{s_{i} / \varepsilon+\alpha_{i} / \varepsilon} f\left(y_{s_{i}}, s\right) \mathrm{d} h_{1}(s)-f_{0}\left(y_{s_{i}}\right)\right|
\end{gathered}
$$

For each $i=0,1,2, \ldots, m-1$, define

$$
\beta_{i}=\frac{\varepsilon}{\alpha_{i}} \int_{s_{i} / \varepsilon}^{s_{i} / \varepsilon+\alpha_{i} / \varepsilon} f\left(y_{s_{i}}, s\right) \mathrm{d} h_{1}(s)-f_{0}\left(y_{s_{i}}\right)
$$

and let $\beta=\max \left\{\left|\beta_{i}\right| ; i=0,1,2, \ldots, m-1\right\}$. Then

$$
\sum_{i=0}^{m-1} \alpha_{i}\left|\beta_{i}\right| \leq \beta \sum_{i=0}^{m-1} \alpha_{i}=\beta \sum_{i=0}^{m-1}\left(s_{i+1}-s_{i}\right)=\beta t<\beta b
$$


and, hence,

$$
\left|\varepsilon \int_{0}^{t} f\left(y_{s}, \frac{s}{\varepsilon}\right) \mathrm{d} h_{1}\left(\frac{s}{\varepsilon}\right)-\int_{0}^{t} f_{0}\left(y_{s}\right) d s\right|<2 \varepsilon D L K b+\beta b .
$$

By Corollary 7.2, $\beta$ tends to zero as $\varepsilon$ tends to zero. Also, $\beta$ depends on $\varepsilon$. Thus, taking $\mu=2 \varepsilon D M K b+\beta b$, then $\mu$ tends to zero as $\varepsilon \rightarrow 0^{+}$and we obtain the inequality

$$
\left|\varepsilon \int_{0}^{t} f\left(y_{s}, \frac{s}{\varepsilon}\right) \mathrm{d} h_{1}\left(\frac{s}{\varepsilon}\right)-\int_{0}^{t} f_{0}\left(y_{s}\right) d s\right|<\mu,
$$

which completes the proof.

The next result is our non-periodic averaging method for measure functional differential equations. This is an original result which can be found in [34].

Theorem 7.4. Consider the measure functional differential equation (7.1.5), where $\phi \in$ $G^{-}\left([-r, 0], \mathbb{R}^{n}\right)$ and $f$ satisfies conditions $\left(H_{1}\right)$ and $\left(H_{2}\right)$. Moreover, assume that the function $h_{1}$ satisfies condition $\left(H_{5}\right), h_{2}$ satisfies condition $\left(H_{6}\right)$ and $g$ satisfies conditions $\left(H_{3}\right)$ and $\left(H_{4}\right)$. Consider the averaged functional differential equation (7.1.7), where $f_{0}$ is given by (7.1.6). Suppose $[0, \bar{b})$ is the maximal interval of existence of solution of (7.1.5) and $[0, b)$ is the maximal interval of existence of solution of (7.1.7). Assume that $x^{\varepsilon}$ is a maximal solution of (7.1.5) and $y$ is a maximal solution of (7.1.7). Let $L>0$ be such that $L<\min (\bar{b}, b)$. Then, for every $\eta>0$, there exists $\varepsilon_{0}>0$ such that for $\varepsilon \in\left(0, \varepsilon_{0}\right]$, the inequality

$$
\left|x^{\varepsilon}(t)-y(t)\right|<\eta
$$

holds for every $t \in[0, L]$.

Proof. We follow the ideas of [35], Theorem 3.1. By hypothesis, for each $t \in[0, L]$, the equalities

$$
x^{\varepsilon}(t)=\phi(0)+\varepsilon \int_{0}^{t} f\left(\left(x^{\varepsilon}\right)_{s}, \frac{s}{\varepsilon}\right) \mathrm{d} h_{1}\left(\frac{s}{\varepsilon}\right)+\varepsilon^{2} \int_{0}^{t} g\left(\left(x^{\varepsilon}\right)_{s}, \frac{s}{\varepsilon}, \varepsilon\right) \mathrm{d} h_{2}\left(\frac{s}{\varepsilon}\right)
$$

and

$$
y(t)=\phi(0)+\int_{0}^{t} f_{0}\left(y_{s}\right) d s
$$

hold. Then, using conditions $\left(H_{2}\right),\left(H_{4}\right)$ and $\left(H_{5}\right)$ and by the Lemma 7.3 , we obtain

$$
\left|x^{\varepsilon}(t)-y(t)\right|=
$$




$$
\begin{gathered}
=\left|\varepsilon \int_{0}^{t} f\left(\left(x^{\varepsilon}\right)_{s}, \frac{s}{\varepsilon}\right) \mathrm{d} h_{1}\left(\frac{s}{\varepsilon}\right)+\varepsilon^{2} \int_{0}^{t} g\left(\left(x^{\varepsilon}\right)_{s}, \frac{s}{\varepsilon}, \varepsilon\right) \mathrm{d} h_{2}\left(\frac{s}{\varepsilon}\right)-\int_{0}^{t} f_{0}\left(y_{s}\right) d s\right| \\
\leq \varepsilon \int_{0}^{t}\left|f\left(\left(x^{\varepsilon}\right)_{s}, \frac{s}{\varepsilon}\right)-f\left(y_{s}, \frac{s}{\varepsilon}\right)\right| \mathrm{d} h_{1}\left(\frac{s}{\varepsilon}\right)+\left|\varepsilon \int_{0}^{t} f\left(y_{s}, \frac{s}{\varepsilon}\right) \mathrm{d} h_{1}\left(\frac{s}{\varepsilon}\right)-\int_{0}^{t} f_{0}\left(y_{s}\right) d s\right| \\
+\varepsilon^{2} \int_{0}^{t}\left|g\left(\left(x^{\varepsilon}\right)_{s}, \frac{s}{\varepsilon}, \varepsilon\right)\right| \mathrm{d} h_{2}\left(\frac{s}{\varepsilon}\right) \\
\leq M \int_{0}^{t}\left\|\left(x^{\varepsilon}\right)_{s}-y_{s}\right\|_{\infty} \mathrm{d} h_{1}\left(\frac{s}{\varepsilon}\right)+\left|\varepsilon \int_{0}^{t} f\left(y_{s}, \frac{s}{\varepsilon}\right) \mathrm{d} h_{1}\left(\frac{s}{\varepsilon}\right)-\int_{0}^{t} f_{0}\left(y_{s}\right) d s\right| \\
+\varepsilon^{2} C\left[h_{2}\left(\frac{t}{\varepsilon}\right)-h_{2}(0)\right]<M \int_{0}^{t}\left\|\left(x^{\varepsilon}\right)_{s}-y_{s}\right\|_{\infty} \mathrm{d} h_{1}\left(\frac{s}{\varepsilon}\right)+\mu+\varepsilon C t N \\
\leq M \int_{0}^{t}\left\|\left(x^{\varepsilon}\right)_{s}-y_{s}\right\|_{\infty} \mathrm{d} h_{1}\left(\frac{s}{\varepsilon}\right)+\mu+\varepsilon C L N,
\end{gathered}
$$

where we used the fact that condition $\left(H_{6}\right)$ implies

$$
\varepsilon^{2} C\left[h_{2}\left(\frac{t}{\varepsilon}\right)-h_{2}(0)\right]=\varepsilon t C \frac{\varepsilon}{t}\left[h_{2}\left(\frac{t}{\varepsilon}\right)-h_{2}(0)\right] \leq \varepsilon t C N
$$

Since $\left(x^{\varepsilon}\right)_{0}=\phi=y_{0}$, we have

$\left\|\left(x^{\varepsilon}\right)_{s}-y_{s}\right\|_{\infty}=\sup _{\theta \in[-r, 0]}\left|x^{\varepsilon}(s+\theta)-y(s+\theta)\right|=\sup _{\sigma \in[-r, s]}\left|x^{\varepsilon}(\sigma)-y(\sigma)\right| \leq \sup _{\sigma \in[0, s]}\left|x^{\varepsilon}(\sigma)-y(\sigma)\right|$, since

$$
\sup _{\sigma \in[-r, 0]}\left|x^{\varepsilon}(\sigma)-y(\sigma)\right|=0
$$

by the definition of initial condition. Therefore, we obtain

$$
\left|x^{\varepsilon}(t)-y(t)\right| \leq M \int_{0}^{t} \sup _{\sigma \in[0, s]}\left|x^{\varepsilon}(\sigma)-y(\sigma)\right| \mathrm{d} h_{1}\left(\frac{s}{\varepsilon}\right)+\mu+\varepsilon C L N .
$$

Also, since the right-hand side of (7.1.13) is increasing, we have

$$
\sup _{\tau \in[0, t]}\left|x^{\varepsilon}(\tau)-y(\tau)\right| \leq M \int_{0}^{t} \sup _{\sigma \in[0, s]} \varepsilon\left|x^{\varepsilon}(\sigma)-y(\sigma)\right| \mathrm{d} h_{1}\left(\frac{s}{\varepsilon}\right)+\mu+\varepsilon C L N .
$$

Then, by the Gronwall's inequality for the Kurzweil-Henstock-Stieltjes integral (Theorem $2.10)$, we obtain

$$
\sup _{\tau \in[0, t]}\left|x^{\varepsilon}(\tau)-y(\tau)\right| \leq e^{\varepsilon M\left(h_{1}(t / \varepsilon)-h_{1}(0)\right)}(\mu+\varepsilon C L N)
$$


Finally,

$$
\sup _{\tau \in[0, t]}\left|x^{\varepsilon}(\tau)-y(\tau)\right| \leq e^{K L M}(\mu+\varepsilon C L N),
$$

since, by hypothesis $\left(H_{5}\right)$, we have

$$
\varepsilon M\left(h_{1}(t / \varepsilon)-h_{1}(0)\right)=t M \frac{\left(h_{1}(t / \varepsilon)-h_{1}(0)\right)}{\frac{t}{\varepsilon}} \leq t M K \leq L M K .
$$

Let $\eta=e^{K L M}(\mu+\varepsilon C L K)$. Then,

$$
\sup _{\tau \in[0, t]}\left|x^{\varepsilon}(\tau)-y(\tau)\right| \leq \eta
$$

where $\eta$ can be taken sufficiently small by the arbitrariness of $\varepsilon$ and the theorem is proved.

A non-periodic averaging result for system

$$
\left\{\begin{array}{l}
D y=\varepsilon f\left(y_{t}, t\right) D h_{1}(t)+\varepsilon^{2} g\left(y_{t}, t, \varepsilon\right) D h_{2}(t) \\
y_{0}=\phi
\end{array}\right.
$$

follows next as a consequence of Theorem 7.4.

Corollary 7.5. Consider the measure functional differential equation (7.1.14) where $\phi \in$ $G^{-}\left([-r, 0], \mathbb{R}^{n}\right)$ and $f$ satisfies conditions $\left(H_{1}\right)$ and $\left(H_{2}\right)$. Moreover, assume that the function $h_{1}$ satisfies condition $\left(H_{5}\right), h_{2}$ satisfies condition $\left(H_{6}\right)$ and $g$ satisfies conditions $\left(H_{3}\right)$ and $\left(H_{4}\right)$. Consider the averaged functional differential equation

$$
\left\{\begin{array}{l}
\dot{x}=f_{0}\left(x_{t}\right) \\
x_{0}=\phi
\end{array}\right.
$$

where $f_{0}$ is given by

$$
f_{0}(\psi)=\lim _{T \rightarrow \infty} \frac{1}{T} \int_{0}^{T} f(\psi, s) \mathrm{d} h_{1}(s) .
$$

Let $x^{\varepsilon}$ and $y$ be solutions of (7.1.14) and (7.1.15) on $[0, L / \varepsilon]$, respectively. Then, for every $\eta$, there exists $a \varepsilon_{0}>0$ such that

$$
\left|x^{\varepsilon}(t)-y(t)\right|<\eta
$$

holds for every $t \in[0, L / \varepsilon]$ and $\varepsilon \in\left(0, \varepsilon_{0}\right]$. 
Now, we present a lemma which will be essential to prove the result following it. Such lemma can be found in [99], Lemma 5.5.

Lemma 7.6. Consider a time scale $\mathbb{T}$ such that $\sup \mathbb{T}=\infty$ and $\lim _{t \rightarrow \infty} \mu(t) / t=0$, a number $t_{0} \in \mathbb{T}$ and a function $H:\left[t_{0}, \infty\right)_{\mathbb{T}} \rightarrow \mathbb{R}^{n}$ such that

$$
\lim _{t \rightarrow \infty, t \in \mathbb{T}} \frac{H(t)}{t}=A
$$

Then,

$$
\lim _{t \rightarrow \infty} \frac{H^{*}(t)}{t}=A
$$

Proof. Given an arbitrary $\varepsilon>0$, we can find $T>\max \left(t_{0}, 0\right)$ such that for every $t \geq T$, we obtain

$$
\left|\frac{H(t)}{t}-A\right|<\varepsilon \quad \text { and } \quad \frac{\mu(t)}{t}<\varepsilon
$$

Then

$$
\left|\frac{H^{*}(t)}{t^{*}}-A\right|<\varepsilon \quad \text { and } \quad\left|H^{*}(t)-A t^{*}\right|<\varepsilon t^{*} .
$$

Also, we get

$\left|H^{*}(t)-A t\right|=\left|H\left(t^{*}\right)-A t^{*}+A t^{*}-A t\right| \leq\left|H\left(t^{*}\right)-A t^{*}\right|+|A|\left(t^{*}-t\right)<\varepsilon t^{*}+|A| \mu\left(\rho\left(t^{*}\right)\right)$.

Now, suppose $t$ is such that $\rho\left(t^{*}\right) \geq T$. Then

$$
\begin{aligned}
& \left|\frac{H^{*}(t)}{t}-A\right|<\frac{\varepsilon t^{*}}{t}+\frac{|A| \mu\left(\rho\left(t^{*}\right)\right)}{t} \leq \frac{\varepsilon t^{*}}{\rho\left(t^{*}\right)}+\frac{|A| \mu\left(\rho\left(t^{*}\right)\right)}{\rho\left(t^{*}\right)} \\
\leq & \frac{\varepsilon \rho\left(t^{*}\right)+\mu\left(\rho\left(t^{*}\right)\right)}{\rho\left(t^{*}\right)}+\frac{|A| \mu\left(\rho\left(t^{*}\right)\right)}{\rho\left(t^{*}\right)}<\varepsilon+\varepsilon+|A| \varepsilon=\varepsilon(2+|A|)
\end{aligned}
$$

and the conclusion follows.

The following result will also be useful to prove our results. It can be found in [99], Corollary 5.6.

Lemma 7.7. If $\sup \mathbb{T}=\infty, \lim _{t \rightarrow \infty} \mu(t) / t=0$ and $h:\left[t_{0}, \infty\right)_{\mathbb{T}} \rightarrow \mathbb{R}^{n}$ is $\Delta$-integrable on every compact subinterval of $\left[t_{0}, \infty\right)_{\mathbb{T}}$, then

$$
\lim _{T \rightarrow \infty} \frac{1}{T} \int_{t_{0}}^{t_{0}+T} h^{*}(s) d \eta(s)=\lim _{T \rightarrow \infty} \frac{1}{t_{0}+T \in \mathbb{T}} \frac{1}{T} \int_{t_{0}}^{t_{0}+T} h(s) d \eta(s)
$$

where $\eta(t)=t^{*}$, provided the limit on the right-hand side exists. 
Proof. Let $H(u)=\int_{t_{0}}^{u} h(s) \Delta s, u \in\left[t_{0}, \infty\right)_{\mathbb{T}}$. Then,

$$
\begin{gathered}
\lim _{T \rightarrow \infty} \frac{1}{T} \int_{t_{0}}^{t_{0}+T} h^{*}(s) \mathrm{d} g(s)=\lim _{T \rightarrow \infty} \frac{H^{*}\left(t_{0}+T\right)}{T}=\lim _{T \rightarrow \infty} \frac{H^{*}\left(t_{0}+T\right)}{t_{0}+T} \frac{t_{0}+T}{T} \\
=\lim _{T \rightarrow \infty} \frac{H^{*}\left(t_{0}+T\right)}{t_{0}+T}=\lim _{u \rightarrow \infty} \frac{H^{*}(u)}{u}=\lim _{u \rightarrow \infty, u \in \mathbb{T}} \frac{H(u)}{u} \\
=\lim _{T \rightarrow \infty, t_{0}+T \in \mathbb{T}} \frac{H\left(t_{0}+T\right)}{t_{0}+T}=\lim _{T \rightarrow \infty, t_{0}+T \in \mathbb{T}} \frac{H\left(t_{0}+T\right)}{T} \frac{T}{t_{0}+T} \\
=\lim _{T \rightarrow \infty, t_{0}+T \in \mathbb{T}} \frac{H\left(t_{0}+T\right)}{T}=\lim _{T \rightarrow \infty, t_{0}+T \in \mathbb{T}} \frac{1}{T} \int_{t_{0}}^{t_{0}+T} h(s) \Delta s .
\end{gathered}
$$

The next result is a non-periodic averaging principle for functional dynamic equations on time scales. Such result is new and is contained in [35].

Theorem 7.8. Let $\mathbb{T}$ be a time scale with $\sup \mathbb{T}=\infty,\left[t_{0}-r, \infty\right)_{\mathbb{T}}$ a time scale interval, $t_{0} \in \mathbb{T}, \varepsilon_{0}>0, L>0, B \subset \mathbb{R}^{n}$ and $\lim _{t \rightarrow \infty} \mu(t) / t=0$. Consider a function $f$ : $G^{-}([-r, 0], B) \times\left[t_{0}, \infty\right)_{\mathbb{T}} \rightarrow \mathbb{R}^{n}$ and a bounded function $g: G^{-}([-r, 0], B) \times\left[t_{0}, \infty\right)_{\mathbb{T}} \times$ $\left(0, \varepsilon_{0}\right] \rightarrow \mathbb{R}^{n}$ such that the following conditions are satisfied:

(i) For every $b>t_{0}$ and $y \in G\left(\left[t_{0}-r, b\right], B\right)$, the functions $t \mapsto f\left(y_{t}, t\right)$ and $t \mapsto g\left(y_{t}, t, \varepsilon\right)$ are regulated on $\left[t_{0}, b\right]_{\mathbb{T}}$.

(ii) For every $b>t_{0}$ and $y \in C\left(\left[t_{0}-r, b\right]_{\mathbb{T}}, B\right)$, the functions $t \mapsto f\left(y_{t}^{*}, t\right)$ and $t \mapsto$ $g\left(y_{t}^{*}, t, \varepsilon\right)$ are $r d$-continuous on $\left[t_{0}, b\right]_{\mathbb{T}}$.

(iii) There is a constant $M>0$ such that for $x, y \in G^{-}([-r, 0], B)$ and $u_{1}, u_{2} \in\left[t_{0}, \infty\right)_{\mathbb{T}}$,

$$
\left|\int_{u_{1}}^{u_{2}}[f(x, s)-f(y, s)] \Delta s\right| \leq M \int_{u_{1}}^{u_{2}}\|x-y\| \Delta s
$$

(iv) If $y:[-r, 0] \rightarrow B$ is a regulated function, then the integral

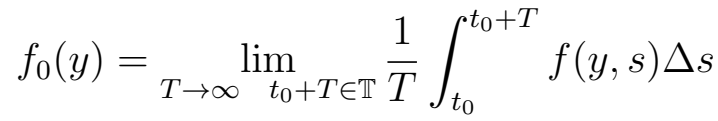

exists. 
Let $\phi \in G^{-}\left(\left[t_{0}-r, t_{0}\right]_{\mathbb{T}}, B\right)$. Suppose for every $\varepsilon \in\left(0, \varepsilon_{0}\right]$, the $\Delta$-integral equation

$$
\left\{\begin{array}{l}
x(t)=x\left(t_{0}\right)+\varepsilon \int_{t_{0}}^{t} f\left(x_{s}^{*}, s\right) \Delta s+\varepsilon^{2} \int_{t_{0}}^{t} g\left(x_{s}^{*}, s, \varepsilon\right) \Delta s \\
x(t)=\phi(t), \quad t \in\left[t_{0}-r, t_{0}\right]_{\mathbb{T}}
\end{array}\right.
$$

has a solution $x_{\varepsilon}:\left[t_{0}-r, t_{0}+L / \varepsilon\right]_{\mathbb{T}} \rightarrow \mathbb{R}^{n}$, and the averaged functional differential equation

$$
\left\{\begin{array}{l}
\dot{y}=f_{0}\left(y_{t}^{*}\right), \\
y_{t_{0}}=\phi_{t_{0}}^{*}
\end{array}\right.
$$

has a solution $y:\left[t_{0}-r, t_{0}+L / \varepsilon\right] \rightarrow \mathbb{R}^{n}$. Then, for every $\eta>0$, there exists $\varepsilon_{0}>0$ such that for $\varepsilon \in\left(0, \varepsilon_{0}\right]$,

$$
\left|x_{\varepsilon}(t)-y(t)\right|<\eta
$$

for every $t \in\left[t_{0}, t_{0}+\frac{L}{\varepsilon}\right]_{\mathbb{T}}$.

Proof. This proof is inspired in the proof of Theorem 5.3 from [99].

Without loss of generality, we assume that $t_{0}=0$. Otherwise, consider the shifted problem with time scale $\widetilde{\mathbb{T}}=\left\{t-t_{0} ; t \in \mathbb{T}\right\}$ and $\widetilde{f}(x, t)=f\left(x, t+t_{0}\right)$ and $\widetilde{g}(x, t, \varepsilon)=$ $g\left(x, t+t_{0}, \varepsilon\right)$.

For $t \in[0, \infty), y \in G^{-}([-r, 0], B)$ and $\varepsilon \in\left(0, \varepsilon_{0}\right]$, define

$$
f^{*}(y, t)=f\left(y, t^{*}\right) \quad \text { and } \quad g^{*}(y, t, \varepsilon)=g\left(y, t^{*}, \varepsilon\right) .
$$

Since $\lim _{t \rightarrow \infty} \mu(t) / t=0$, there are numbers $D>0$ and $\tau \in \mathbb{T}$ such that $\mu(t) / t \leq D$ for every $t \in[\tau, \infty)_{\mathbb{T}}$. Note that

$$
\mu\left(\rho\left(t^{*}\right)\right)=\sigma\left(\rho\left(t^{*}\right)\right)-\rho\left(t^{*}\right)=t^{*}-\rho\left(t^{*}\right)
$$

which implies

$$
t^{*}-\mu\left(\rho\left(t^{*}\right)\right)=\rho\left(t^{*}\right) \leq \rho(t) \leq t .
$$

Then, if $t \in \mathbb{R}$ is such that $\rho\left(t^{*}\right) \geq \tau$, we have

$$
t^{*} \leq t+\mu\left(\rho\left(t^{*}\right)\right) \leq t+D \rho\left(t^{*}\right) \leq t+D t=t(D+1)
$$

For every $t \in[0, \infty)$, let $h(t)=t^{*}$. Then, for sufficiently large $T$ and for every $a \geq 0$, 
we have

$$
\frac{h(a+T)-h(a)}{T}=\frac{(a+T)^{*}-a^{*}}{T} \leq \frac{(a+T)(D+1)-a^{*}}{T},
$$

and, consequently,

$$
\limsup _{T \rightarrow \infty} \frac{h(a+T)-h(a)}{T} \leq \limsup _{T \rightarrow \infty} \frac{(a+T)(D+1)-a^{*}}{T}=D+1 .
$$

Therefore condition $\left(H_{5}\right)$ is satisfied for the function $h$.

By Theorems 4.11 and 4.12 and Lemma 7.7, we have

$$
\begin{aligned}
f_{0}(y) & =\lim _{T \rightarrow \infty} \frac{1}{T} \int_{0}^{T} f(y, s) \Delta s=\lim _{T \rightarrow \infty} \frac{1}{T} \int_{0}^{T} f\left(y, s^{*}\right) \mathrm{d} h(s) \\
& =\lim _{T \rightarrow \infty} \frac{1}{T} \int_{0}^{T} f\left(y, s^{*}\right) \mathrm{d} h(s)=\lim _{T \rightarrow \infty} \frac{1}{T} \int_{0}^{T} f^{*}(y, s) \mathrm{d} h(s)
\end{aligned}
$$

for every $y \in G^{-}([-r, 0], B)$. Since $x_{\varepsilon}$ is a solution of (7.1.16), Theorem 4.13 implies $x_{\varepsilon}^{*}$ is a solution of the system

$$
\left\{\begin{array}{l}
D x^{*}=\varepsilon f^{*}\left(x_{t}^{*}, t\right) D h(t)+\varepsilon^{2} g^{*}\left(x_{t}^{*}, t, \varepsilon\right) D h(t), \\
x_{0}^{*}=\phi_{0}^{*}
\end{array}\right.
$$

By Lemma 4.24, $f$ fulfills conditions $\left(H_{1}\right)$ and $\left(H_{2}\right)$ and $g$ fulfills the conditions $\left(H_{3}\right)$ and $\left(H_{4}\right)$. Thus, all hypotheses of Corollary 7.5 are satisfied. Then, for every $\eta>0$, there exists $\varepsilon_{0}>0$ such that, for every $\varepsilon \in\left(0, \varepsilon_{0}\right]$ and every $t \in[0, L / \varepsilon]$, the inequality

$$
\left|x_{\varepsilon}^{*}(t)-y(t)\right| \leq \eta
$$

holds, where $y$ is a solution of (7.1.17). The proof is complete after observing that $x_{\varepsilon}^{*}(t)=x_{\varepsilon}(t)$ for $t \in[0, L / \varepsilon]_{\mathbb{T}}$.

\subsubsection{Impulsive measure FDEs and impulsive functional dynamic equations on time scales}

In this section, we present original non-periodic averaging theorems for impulsive measure FDEs and for impulsive functional dynamic equations on time scales.

We start by presenting a non-periodic averaging method for impulsive measure FDEs. 
Theorem 7.9. Consider the impulsive measure functional differential equation

$$
\left\{\begin{array}{l}
y(t)=y\left(t_{0}\right)+\varepsilon \int_{t_{0}}^{t} f\left(y_{s}, s\right) \mathrm{d} h(s)+\varepsilon^{2} \int_{t_{0}}^{t} g\left(y_{s}, s, \varepsilon\right) \mathrm{d} h(s)+\varepsilon \sum_{\substack{k \in \mathbb{N}, t_{k}<t}} I_{k}\left(y\left(t_{k}\right)\right), \\
y_{0}=\phi
\end{array}\right.
$$

where $\phi \in G^{-}\left([-r, 0], \mathbb{R}^{n}\right)$ and the function $f: G^{-}\left([-r, 0], \mathbb{R}^{n}\right) \times[0, \infty) \rightarrow \mathbb{R}^{n}$ satisfies conditions $\left(H_{1}\right)$ and $\left(H_{2}\right)$. Moreover, assume that the function $h:[0, \infty) \rightarrow \mathbb{R}$ satisfies condition $\left(H_{5}\right)$ and the function $g: G^{-}\left([-r, 0], \mathbb{R}^{n}\right) \times[0, \infty) \times\left(0, \varepsilon_{0}\right] \rightarrow \mathbb{R}^{n}$ satisfies condition $\left(H_{3}\right)$ and is bounded. Also, suppose the impulse operators $I_{k}: \mathbb{R}^{n} \rightarrow \mathbb{R}^{n}$ satisfy conditions $\left(A^{*}\right)$ and $\left(B^{*}\right)$, with the same Lipschitzian and boundedness constant for all $k \in \mathbb{N}$. Further, suppose the integral

$$
f_{0}(\psi)=\lim _{T \rightarrow \infty} \frac{1}{T} \int_{0}^{T} f(\psi, s) \mathrm{d} h(s)
$$

exists, for every $\psi \in G^{-}\left([-r, 0], \mathbb{R}^{n}\right)$ and denote

$$
I_{0}(y)=\lim _{T \rightarrow \infty} \frac{1}{T} \sum_{0 \leq t_{k}<T} I_{k}(y), \quad y \in B .
$$

Consider the averaged functional differential equation

$$
\left\{\begin{array}{l}
\dot{x}=f_{0}\left(x_{t}^{*}\right)+I_{0}(y) \\
x_{0}=\phi
\end{array}\right.
$$

Let $x^{\varepsilon}$ and $y$ be solutions of (7.1.21) and (7.1.22) on $[0, L / \varepsilon]$ respectively. Then, for every $\eta$, there exists $\varepsilon_{0}>0$ such that the inequality

$$
\left|x^{\varepsilon}(t)-y(t)\right|<\eta
$$

holds for every $t \in[0, L / \varepsilon]$ and every $\varepsilon \in\left(0, \varepsilon_{0}\right]$.

Proof. Define the function $\tilde{h}:[0, \infty) \rightarrow \mathbb{R}$ by

$$
\tilde{h}(t)= \begin{cases}h(t), & t \in\left[0, t_{1}\right], \\ h(t)+c_{k}, & t \in\left(t_{k}, t_{k+1}\right] \text { for some } k \in \mathbb{N}\end{cases}
$$

where the sequence $\left\{c_{k}\right\}_{k=1}^{\infty}$ is chosen in such a way that $\Delta^{+} \tilde{h}\left(t_{k}\right)=1$, for every $k \in \mathbb{N}$. 
Note that the functions $\tilde{h}$ and $\tilde{h}$ are nondecreasing and left-continuous. Also, by condition $\left(H_{5}\right)$ and by the definition of $\tilde{h}$, there exists a constant $K_{1}>0$ such that, for every $\beta \geq 0$, the following inequality

$$
\limsup _{T \rightarrow \infty} \frac{\tilde{h}(T+\beta)-\tilde{h}(\beta)}{T} \leq K_{1}
$$

holds.

According to the assumptions, we have

$$
x^{\varepsilon}(t)=x^{\varepsilon}(0)+\int_{0}^{t}\left[\varepsilon f\left(x_{s}^{\varepsilon}, s\right)+\varepsilon^{2} g\left(x_{s}^{\varepsilon}, s, \varepsilon\right)\right] \mathrm{d} h(s)+\sum_{0 \leq t_{k}<t} \varepsilon I_{k}\left(x^{\varepsilon}\left(t_{k}\right)\right),
$$

for every $\varepsilon \in\left(0, \varepsilon_{0}\right]$ and every $t \in[0, L / \varepsilon]$.

Let

$$
F^{\varepsilon}(y, t)= \begin{cases}\varepsilon f(y, t)+\varepsilon^{2} g(y, t, \varepsilon), & t \notin\left\{t_{1}, t_{2}, \ldots\right\}, \\ \varepsilon I_{k}(y(0)), & t=t_{k} \text { for some } k \in \mathbb{N}\end{cases}
$$

for every $y \in G^{-}\left([-r, 0], \mathbb{R}^{n}\right)$ and every $t \geq 0$. By Theorem 4.16,

$$
x^{\varepsilon}(t)=x^{\varepsilon}(0)+\int_{0}^{t} F^{\varepsilon}\left(x_{s}^{\varepsilon}, s\right) \mathrm{d} \tilde{h}(s),
$$

for every $\varepsilon \in\left(0, \varepsilon_{0}\right]$ and every $t \in[0, L / \varepsilon]$. For $y \in G^{-}\left([-r, 0], \mathbb{R}^{n}\right)$ and $t \geq 0$, we can write

$$
F^{\varepsilon}(y, t)=\varepsilon \tilde{f}(y, t)+\varepsilon^{2} \tilde{g}(y, t, \varepsilon)
$$

where

$$
\tilde{f}(y, t)= \begin{cases}f(y, t), & t \notin\left\{t_{1}, t_{2}, \ldots\right\} \\ I_{k}(y(0)), & t=t_{k} \text { for some } k \in \mathbb{N}\end{cases}
$$

and

$$
\tilde{g}(y, t, \varepsilon)= \begin{cases}g(y, t, \varepsilon), & t \notin\left\{t_{1}, t_{2}, \ldots\right\} \\ 0, & t=t_{k} \text { for some } k \in \mathbb{N}\end{cases}
$$

It follows from (7.1.23) and (7.1.24) that, for every $\varepsilon \in\left(0, \varepsilon_{0}\right]$, the function $x^{\varepsilon}$ : 
$[-r, L / \varepsilon] \rightarrow B$ is a solution of the initial value problem

$$
\left\{\begin{array}{l}
x(t)=x(0)+\varepsilon \int_{0}^{t} \tilde{f}\left(x_{s}, s\right) \mathrm{d} \tilde{h}(s)+\varepsilon^{2} \int_{0}^{t} \tilde{g}\left(x_{s}, s, \varepsilon\right) \mathrm{d} \tilde{h}(s) \\
x_{0}=\phi
\end{array}\right.
$$

By the definition of the function $\tilde{f}$ and by the hypotheses, it follows from Lemma 4.18 that the function $\tilde{f}$ satisfies condition $\left(H_{2}\right)$.

Using Lemma 4.15, we have

$$
\begin{aligned}
\int_{0}^{T} \tilde{f}(x, s) \mathrm{d} \tilde{h}(s) & =\int_{0}^{T} f(x, s) \mathrm{d} h(s)+\sum_{k ; 0 \leq t_{k}<T} \tilde{f}\left(x, t_{k}\right) \Delta^{+} \tilde{h}\left(t_{k}\right) \\
& =\int_{0}^{T} f(x, s) \mathrm{d} h(s)+\sum_{k ; 0 \leq t_{k}<T} I_{k}(x(0)),
\end{aligned}
$$

for every $x \in G^{-}\left([-r, 0], \mathbb{R}^{n}\right)$. Consequently, the function $\tilde{f}_{0}: G^{-}\left([-r, 0], \mathbb{R}^{n}\right) \rightarrow \mathbb{R}^{n}$ defined by

$$
\tilde{f}_{0}(x)=\lim _{T \rightarrow \infty} \frac{1}{T} \int_{0}^{T} \tilde{f}(x, s) \mathrm{d} \tilde{h}(s), \quad x \in G^{-}\left([-r, 0], \mathbb{R}^{n}\right),
$$

satisfies

$$
\tilde{f}_{0}(x)=\lim _{T \rightarrow \infty} \frac{1}{T} \int_{0}^{T} f(x, s) \mathrm{d} h(s)+\lim _{T \rightarrow \infty} \frac{1}{T} \sum_{k ; 0 \leq t_{k}<T} I_{k}(x(0))=f_{0}(x)+I_{0}(x(0)),
$$

for $x \in G^{-}\left([-r, 0], \mathbb{R}^{n}\right)$.

Finally, by Corollary 7.5, for every $\eta>0$, there exists $\varepsilon_{0}>0$ such that

$$
\left|x^{\varepsilon}(t)-y^{\varepsilon}(t)\right| \leq \eta
$$

holds, for every $\varepsilon \in\left(0, \varepsilon_{0}\right]$ and every $t \in[0, L / \varepsilon]$ and the proof is finished.

The following original result is our non-periodic averaging theorem for impulsive functional dynamic equations on time scales. Its proof is similar to the proof of Theorem 7.8 from the previous section. We include it here though.

Theorem 7.10. Let $\mathbb{T}$ be a time scale with $\sup \mathbb{T}=\infty,\left[t_{0}-r, \infty\right)_{\mathbb{T}}$ a time scale interval, $t_{0} \in \mathbb{T}, \varepsilon_{0}>0, L>0, B \subset \mathbb{R}^{n}$ and $\lim _{t \rightarrow \infty} \mu(t) / t=0$. Let $\left\{t_{k}\right\}_{k \in \mathbb{N}} \in \mathbb{T}$ be a sequence of moments of impulses which are right-dense and satisfy $t_{0} \leq t_{1}<t_{2}<\ldots<t_{m}<\ldots$. 
Also, consider the impulse operators $I_{k}: B \rightarrow \mathbb{R}^{n}, k \in \mathbb{N}$, which satisfy conditions $\left(A^{*}\right)$ and $\left(B^{*}\right)$. Suppose the function $f: G^{-}([-r, 0], B) \times\left[t_{0}, \infty\right)_{\mathbb{T}} \rightarrow \mathbb{R}^{n}$ satisfies conditions $\left(H_{1}\right)$ and $\left(H_{2}\right)$ and the function $g: G^{-}([-r, 0], B) \times\left[t_{0}, \infty\right)_{\mathbb{T}} \times\left(0, \varepsilon_{0}\right] \rightarrow \mathbb{R}^{n}$ is bounded. Moreover, suppose the following conditions are satisfied:

(i) For $b>t_{0}$ and $y \in G^{-}\left(\left[t_{0}-r, b\right], B\right)$, the integrals $\int_{t_{0}}^{b} f\left(y_{s}, s\right) \Delta s$ and $\int_{t_{0}}^{b} g\left(y_{s}, s, \varepsilon\right) \Delta s$ exist.

(ii) If $y:[-r, 0] \rightarrow B$ is a regulated function, then the integral

$$
f_{0}(y)=\lim _{T \rightarrow \infty} \frac{1}{t_{0}+T \in \mathbb{T}} \int_{t_{0}}^{t_{0}+T} f(y, s) \Delta s
$$

exists.

(iii) Denote

$$
I_{0}(y)=\lim _{T \rightarrow \infty} \frac{1}{T} \sum_{k ; 0 \leq t_{k}<T} I_{k}(y), \quad y \in B
$$

Let $\phi \in G^{-}\left(\left[t_{0}-r, t_{0}\right]_{\mathbb{T}}, B\right)$. Suppose for every $\varepsilon \in\left(0, \varepsilon_{0}\right]$, the $\Delta$-integral equation

$$
\left\{\begin{array}{l}
x(t)=x\left(t_{0}\right)+\varepsilon \int_{t_{0}}^{t} f\left(x_{s}^{*}, s\right) \Delta s+\varepsilon^{2} \int_{t_{0}}^{t} g\left(x_{s}^{*}, s, \varepsilon\right) \Delta s+\varepsilon \sum_{\substack{k \in \mathbb{N} \\
t_{0} \leq t_{k}<t}} I_{k}\left(x\left(t_{k}\right)\right), \\
x(t)=\phi(t), \quad t \in\left[t_{0}-r, t_{0}\right]_{\mathbb{T}}
\end{array}\right.
$$

has a solution $x_{\varepsilon}:\left[t_{0}-r, t_{0}+\frac{L}{\varepsilon}\right]_{\mathbb{T}} \rightarrow \mathbb{R}^{n}$ and the averaged functional differential equation

$$
\left\{\begin{array}{l}
\dot{y}=f_{0}\left(y_{t}^{*}\right)+I_{0}(y) \\
y_{t_{0}}=\phi_{t_{0}}
\end{array}\right.
$$

has a solution $y:\left[t_{0}-r, t_{0}+\frac{L}{\varepsilon}\right] \rightarrow \mathbb{R}^{n}$. Then, for every $\eta>0$, there exists $\varepsilon_{0}>0$ such that for every $\varepsilon \in\left(0, \varepsilon_{0}\right]$,

$$
\left|x_{\varepsilon}(t)-y(t)\right|<\eta
$$

for $t \in\left[t_{0}, t_{0}+\frac{M}{\varepsilon}\right]_{\mathbb{T}}$.

Proof. Using the same argument as in the proof of Theorem 7.8, we assume, without loss of generality, that $t_{0}=0$. 
Given $t \in[0, \infty), y \in G^{-}\left(\left[t_{0}-r, t_{0}\right]_{\mathbb{T}}, B\right)$ and $\varepsilon \in\left(0, \varepsilon_{0}\right]$, define the following functions:

$$
f^{*}(y, t)=f\left(y, t^{*}\right) \quad \text { and } \quad g^{*}(y, t, \varepsilon)=g\left(y, t^{*}, \varepsilon\right) .
$$

Since $\lim _{t \rightarrow \infty} \mu(t) / t=0$, there are numbers $D>0$ and $\tau \in \mathbb{T}$ such that $\mu(t) / t \leq D$ for every $t \in[\tau, \infty)_{\mathbb{T}}$. Moreover,

$$
\mu\left(\rho\left(t^{*}\right)\right)=\sigma\left(\rho\left(t^{*}\right)\right)-\rho\left(t^{*}\right)=t^{*}-\rho\left(t^{*}\right)
$$

which implies

$$
t^{*}-\mu\left(\rho\left(t^{*}\right)\right)=\rho\left(t^{*}\right) \leq \rho(t) \leq t .
$$

Then, if $t \in \mathbb{R}$ is such that $\rho\left(t^{*}\right) \geq \tau$, we have

$$
t^{*} \leq t+\mu\left(\rho\left(t^{*}\right)\right) \leq t+D \rho\left(t^{*}\right) \leq t+D t=t(D+1)
$$

For every $t \in[0, \infty)$, let $h(t)=t^{*}$. Then, for sufficiently large $T$ and for every $a \geq 0$, we have

$$
\frac{h(a+T)-h(a)}{T}=\frac{(a+T)^{*}-a^{*}}{T} \leq \frac{(a+T)(D+1)-a^{*}}{T},
$$

and, therefore, applying the $\lim \sup$ as $T \rightarrow \infty$ in both sides of equation (7.1.29), it follows immediately that condition $\left(H_{5}\right)$ is satisfied for the function $h$.

By Theorems 4.11 and 4.12 and Lemma 7.7, we obtain

$$
\begin{aligned}
f_{0}(y) & =\lim _{T \rightarrow \infty} \frac{1}{T} \int_{0}^{T} f(y, s) \Delta s=\lim _{T \rightarrow \infty} \frac{1}{T} \int_{0}^{T} f\left(y, s^{*}\right) \mathrm{d} h(s)= \\
& =\lim _{T \rightarrow \infty} \frac{1}{T} \int_{0}^{T} f\left(y, s^{*}\right) \mathrm{d} h(s)=\lim _{T \rightarrow \infty} \frac{1}{T} \int_{0}^{T} f^{*}(y, s) \mathrm{d} h(s),
\end{aligned}
$$

for every $y \in G^{-}([-r, 0], B)$. Then, since $x_{\varepsilon}$ is a solution of $(7.1 .27)$, it follows from Theorem 4.23 that $x_{\varepsilon}^{*}$ is a solution of

$$
\left\{\begin{array}{l}
x_{\varepsilon}^{*}(t)=x_{\varepsilon}^{*}(0)+\varepsilon \int_{0}^{t} f^{*}\left(\left(x_{\varepsilon}^{*}\right)_{s}, s\right) \mathrm{d} h(s)+\varepsilon^{2} \int_{0}^{t} g^{*}\left(\left(x_{\varepsilon}^{*}\right)_{s}, s, \varepsilon\right) \mathrm{d} h(s)+\varepsilon \sum_{\substack{k \in \mathbb{N} \\
t_{k}<t}} I_{k}\left(x_{\varepsilon}^{*}\left(t_{k}\right)\right), \\
x_{0}^{*}=\phi_{0} .
\end{array}\right.
$$

By Lemma 4.24, it follows that the function $f^{*}$ satisfies conditions $\left(H_{1}\right)$ and $\left(H_{2}\right)$ and the function $g^{*}$ satisfies the boundedness condition. Thus, all hypotheses of Theorem 7.9 
are satisfied and, hence, for every $\eta>0$, there exists $\varepsilon_{0}>0$ such that, for every $\varepsilon \in\left(0, \varepsilon_{0}\right]$ and $t \in[0, L / \varepsilon]$, the inequality

$$
\left|x_{\varepsilon}^{*}(t)-y_{\varepsilon}(t)\right| \leq \eta
$$

holds, where $y_{\varepsilon}$ is a solution of (7.1.28). Since $x_{\varepsilon}^{*}(t)=x_{\varepsilon}(t)$ for $t \in[0, L / \varepsilon]_{\mathbb{T}}$, the result follows.

\subsection{Periodic averaging principles}

In this section, we focus our attention on periodic averaging principles for various types of differential equations.

Classical periodic averaging theorems for ordinary differential equations are concerned with the initial value problem

$$
\left\{\begin{array}{l}
\dot{x}=\varepsilon f(x(t), t)+\varepsilon^{2} g(x(t), t, \varepsilon), \\
x\left(t_{0}\right)=x_{0}
\end{array}\right.
$$

where $\varepsilon>0$ is a small parameter, $t_{0}>0, f: \mathbb{R}^{n} \times \mathbb{R} \rightarrow \mathbb{R}^{n}, g: \mathbb{R}^{n} \times \mathbb{R} \times\left[0, \varepsilon_{0}\right) \rightarrow \mathbb{R}^{n}$. Assume that $f$ is $T$-periodic in the second argument. Then, according to the periodic averaging theorem, we can obtain a good approximation of this initial value problem by neglecting the $\varepsilon^{2}$-term and taking the average of $f$ with respect to $t$. In other words, it is commonly considered the autonomous differential equation

$$
\dot{y}=\varepsilon f_{0}(y(t)), \quad y\left(t_{0}\right)=x_{0},
$$

where

$$
f_{0}(y)=\frac{1}{T} \int_{t_{0}}^{t_{0}+T} f(y, t) \mathrm{d} t .
$$

Historically, periodic averaging theorems were really important for the development and progress of the study of celestial mechanic problems. In such problems, usually one deals with periodic functions which allow periodic averaging systems to obtain good approximations between the solutions of nonlinear systems and the solutions of an autonomous averaged system.

Different proofs of periodic averaging theorems can be found in [92], [93], or [102], for instance. These monographs also include many applications and examples. 
This section is divided in three subsections. The first one is devoted to the presentation of a periodic averaging theorem for generalized ordinary differential equations. In the second one, we prove periodic averaging theorems for measure functional differential equations and for functional dynamic equations on time scales. Finally, in the last one, we prove periodic averaging theorems for impulsive measure functional differential equations and for impulsive functional dynamic equations on time scales.

We emphasize that almost all results presented in these subsections are new and they can be found in [84].

\subsubsection{Generalized ODEs}

In this subsection, we prove a periodic averaging theorem for generalized ordinary differential equations. The proof is inspired by a proof of the classical averaging theorem for ordinary differential equations given in [93] (see Theorem 2.8.1 and Lemma 2.8.2 there). Our result is new and can be found in [84].

Theorem 7.11. Let $B \subset \mathbb{R}^{n}, \Omega=B \times[0, \infty), \varepsilon_{0}>0, L>0$. Consider functions $F: \Omega \rightarrow \mathbb{R}^{n}$ and $G: \Omega \times\left(0, \varepsilon_{0}\right] \rightarrow \mathbb{R}^{n}$ which satisfy the following conditions:

1. There exist nondecreasing left-continuous functions $h_{1}, h_{2}:[0, \infty) \rightarrow[0, \infty)$ such that $F$ belongs to the class $\mathcal{F}\left(\Omega, h_{1}\right)$, and for every fixed $\varepsilon \in\left(0, \varepsilon_{0}\right]$, the function $(x, t) \mapsto G(x, t, \varepsilon)$ belongs to the class $\mathcal{F}\left(\Omega, h_{2}\right)$.

2. $F(x, 0)=0$ and $G(x, 0, \varepsilon)=0$, for every $x \in B, \varepsilon \in\left(0, \varepsilon_{0}\right]$.

3. There exists a number $T>0$ and a bounded Lipschitz continuous function $M: B \rightarrow$ $\mathbb{R}^{n}$ such that $F(x, t+T)-F(x, t)=M(x)$, for every $x \in B$ and $t \in[0, \infty)$.

4. There exists a constant $\alpha>0$ such that $h_{1}(i T)-h_{1}((i-1) T) \leq \alpha$, for every $i \in \mathbb{N}$.

5. There exists a constant $\beta>0$ such that $\left|h_{2}(t) / t\right| \leq \beta$, for every $t \geq L / \varepsilon_{0}$.

Let

$$
F_{0}(x)=\frac{F(x, T)}{T}, x \in B
$$

Suppose for every $\varepsilon \in\left(0, \varepsilon_{0}\right]$, the initial value problems

$$
\left\{\begin{array}{l}
\frac{\mathrm{d} x}{\mathrm{~d} \tau}=D\left[\varepsilon F(x, t)+\varepsilon^{2} G(x, t, \varepsilon)\right] \\
x(0)=x_{0}(\varepsilon)
\end{array}\right.
$$


and

$$
\left\{\begin{array}{l}
\dot{y}=\varepsilon F_{0}(y(t)), \\
y(0)=y_{0}(\varepsilon)
\end{array}\right.
$$

have solutions $x_{\varepsilon}, y_{\varepsilon}:\left[0, \frac{L}{\varepsilon}\right] \rightarrow B$, respectively. If there is a constant $J>0$ such that $\left|x_{0}(\varepsilon)-y_{0}(\varepsilon)\right| \leq J \varepsilon$ for every $\varepsilon \in\left(0, \varepsilon_{0}\right]$, then there exists a constant $K>0$ such that

$$
\left|x_{\varepsilon}(t)-y_{\varepsilon}(t)\right| \leq K \varepsilon
$$

for every $\varepsilon \in\left(0, \varepsilon_{0}\right]$ and $t \in\left[0, \frac{L}{\varepsilon}\right]$.

Proof. If $x \in B$, then

$$
\left|F_{0}(x)\right|=\left|\frac{F(x, T)}{T}\right|=\left|\frac{F(x, T)-F(x, 0)}{T}\right|=\frac{|M(x)|}{T} \leq \frac{m}{T}
$$

where $m$ is a bound for $M$. Let $l$ be a Lipschitz constant for $M$. The function $H$ : $B \times[0, \infty) \rightarrow \mathbb{R}^{n}$ given by

$$
H(x, t)=F_{0}(x) t=\frac{F(x, T)}{T} t
$$

satisfies

$$
\begin{aligned}
\left|H\left(x, s_{2}\right)-H\left(x, s_{1}\right)\right| & =\frac{1}{T}\left|F(x, T) s_{2}-F(x, T) s_{1}\right| \\
& =\frac{1}{T}|F(x, T)|\left(s_{2}-s_{1}\right) \leq \frac{m}{T}\left(s_{2}-s_{1}\right)
\end{aligned}
$$

and

$$
\begin{gathered}
\left|H\left(x, s_{2}\right)-H\left(x, s_{1}\right)-H\left(y, s_{2}\right)+H\left(y, s_{1}\right)\right| \\
=\frac{1}{T}\left|F(x, T) s_{2}-F(x, T) s_{1}-F(y, T) s_{2}+F(y, T) s_{1}\right| \\
=\frac{1}{T}|F(x, T)-F(y, T)|\left(s_{2}-s_{1}\right)=\frac{1}{T}|M(x)-M(y)|\left(s_{2}-s_{1}\right) \\
\leq \frac{l}{T}|x-y|\left(s_{2}-s_{1}\right),
\end{gathered}
$$

for every $x, y \in B$ and every $s_{1}, s_{2} \in[0, \infty), s_{1} \leq s_{2}$. It follows that $H$ belongs to the class $\mathcal{F}\left(\Omega, h_{3}\right)$, where $h_{3}(t)=\frac{m+l}{T} t$. 
For every $t \in[0, L / \varepsilon]$, we have

$$
\begin{gathered}
x_{\varepsilon}(t)=x_{0}(\varepsilon)+\varepsilon \int_{0}^{t} D F\left(x_{\varepsilon}(\tau), s\right)+\varepsilon^{2} \int_{0}^{t} D G\left(x_{\varepsilon}(\tau), s, \varepsilon\right), \\
y_{\varepsilon}(t)=y_{0}(\varepsilon)+\varepsilon \int_{0}^{t} F_{0}\left(y_{\varepsilon}(\tau)\right) \mathrm{d} \tau=y_{\varepsilon}(0)+\varepsilon \int_{0}^{t} D\left[F_{0}\left(y_{\varepsilon}(\tau)\right) s\right] .
\end{gathered}
$$

Consequently,

$$
\begin{gathered}
\left|x_{\varepsilon}(t)-y_{\varepsilon}(t)\right|= \\
=\left|x_{0}(\varepsilon)-y_{0}(\varepsilon)+\varepsilon \int_{0}^{t} D F\left(x_{\varepsilon}(\tau), s\right)+\varepsilon^{2} \int_{0}^{t} D G\left(x_{\varepsilon}(\tau), s, \varepsilon\right)-\varepsilon \int_{0}^{t} D\left[F_{0}\left(y_{\varepsilon}(\tau)\right) s\right]\right| \\
\leq J \varepsilon+\varepsilon\left|\int_{0}^{t} D\left[F\left(x_{\varepsilon}(\tau), s\right)-F\left(y_{\varepsilon}(\tau), s\right)\right]\right|+\varepsilon\left|\int_{0}^{t} D\left[F\left(y_{\varepsilon}(\tau), s\right)-F_{0}\left(y_{\varepsilon}(\tau)\right) s\right]\right| \\
+\varepsilon^{2}\left|\int_{0}^{t} D G\left(x_{\varepsilon}(\tau), s, \varepsilon\right)\right| .
\end{gathered}
$$

According to Lemma 2.14, we have the estimate

$$
\varepsilon^{2}\left|\int_{0}^{t} D G\left(x_{\varepsilon}(\tau), s, \varepsilon\right)\right| \leq \varepsilon^{2}\left(h_{2}(t)-h_{2}(0)\right) \leq \varepsilon^{2} h_{2}(L / \varepsilon)=\varepsilon L \frac{h_{2}(L / \varepsilon)}{L / \varepsilon} \leq \varepsilon L \beta .
$$

Also, it follows from Lemma 5.1 that

$$
\left|\int_{0}^{t} D\left[F\left(x_{\varepsilon}(\tau), s\right)-F\left(y_{\varepsilon}(\tau), s\right)\right]\right| \leq \int_{0}^{t}\left|x_{\varepsilon}(s)-y_{\varepsilon}(s)\right| \mathrm{d} h_{1}(s) .
$$

Let $p$ be the largest integer such that $p T \leq t$. Then

$$
\begin{aligned}
\int_{0}^{t} D\left[F\left(y_{\varepsilon}(\tau), s\right)-F_{0}\left(y_{\varepsilon}(\tau)\right) s\right] & =\sum_{i=1}^{p} \int_{(i-1) T}^{i T} D\left[F\left(y_{\varepsilon}(\tau), s\right)-F_{0}\left(y_{\varepsilon}(\tau)\right) s\right] \\
& +\int_{p T}^{t} D\left[F\left(y_{\varepsilon}(\tau), s\right)-F_{0}\left(y_{\varepsilon}(\tau)\right) s\right] .
\end{aligned}
$$

For every $i \in\{1, \ldots, p\}$, we obtain

$$
\begin{array}{r}
\int_{(i-1) T}^{i T} D\left[F\left(y_{\varepsilon}(\tau), s\right)-F_{0}\left(y_{\varepsilon}(\tau)\right) s\right]=\int_{(i-1) T}^{i T} D\left[F\left(y_{\varepsilon}(\tau), s\right)-F\left(y_{\varepsilon}(i T), s\right)\right] \\
-\int_{(i-1) T}^{i T} D\left[F_{0}\left(y_{\varepsilon}(\tau)\right) s-F_{0}\left(y_{\varepsilon}(i T)\right) s\right]+\int_{(i-1) T}^{i T} D\left[F\left(y_{\varepsilon}(i T), s\right)-F_{0}\left(y_{\varepsilon}(i T)\right) s\right] .
\end{array}
$$


We estimate the first integral on the right-hand side of (7.2.4) as follows:

$$
\left|\int_{(i-1) T}^{i T} D\left[F\left(y_{\varepsilon}(\tau), s\right)-F\left(y_{\varepsilon}(i T), s\right)\right]\right| \leq \int_{(i-1) T}^{i T}\left|y_{\varepsilon}(s)-y_{\varepsilon}(i T)\right| \mathrm{d} h_{1}(s) .
$$

Since $y_{\varepsilon}$ satisfies $\dot{y}_{\varepsilon}=\varepsilon F_{0}\left(y_{\varepsilon}(t)\right)$, the mean value theorem gives

$$
\left|y_{\varepsilon}(s)-y_{\varepsilon}(i T)\right| \leq \varepsilon \frac{m}{T}(i T-s) \leq \varepsilon m, \quad s \in[(i-1) T, i T],
$$

and, consequently,

$$
\int_{(i-1) T}^{i T}\left|y_{\varepsilon}(s)-y_{\varepsilon}(i T)\right| \mathrm{d} h_{1}(s) \leq \varepsilon m\left[h_{1}(i T)-h_{1}((i-1) T)\right] \leq \varepsilon m \alpha .
$$

The same procedure applied to the second integral on the right-hand side of (7.2.4) gives

$$
\left|\int_{(i-1) T}^{i T} D\left[F_{0}\left(y_{\varepsilon}(\tau)\right) s-F_{0}\left(y_{\varepsilon}(i T)\right) s\right]\right| \leq \varepsilon m\left[h_{3}(i T)-h_{3}((i-1) T)\right] \leq \varepsilon m(m+l) .
$$

The third integral on the right-hand side of (7.2.4) is zero, because for an arbitrary $y \in B$, we have

$$
\begin{aligned}
\int_{(i-1) T}^{i T} D\left[F(y, s)-F_{0}(y) s\right] & =F(y, i T)-F(y,(i-1) T)-F_{0}(y) T \\
& =M(y)-F(y, T)=0 .
\end{aligned}
$$

Since $p T \leq L / \varepsilon$, we obtain

$$
\left|\sum_{i=1}^{p} \int_{(i-1) T}^{i T} D\left[F\left(y_{\varepsilon}(\tau), s\right)-F_{0}\left(y_{\varepsilon}(\tau)\right) s\right]\right| \leq p \varepsilon m \alpha+p \varepsilon m(m+l) \leq \frac{L m \alpha}{T}+\frac{m(m+l) L}{T} .
$$

Finally, the following estimate is a consequence of Lemma 2.14:

$$
\begin{gathered}
\left|\int_{p T}^{t} D\left[F\left(y_{\varepsilon}(\tau), s\right)-F_{0}\left(y_{\varepsilon}(\tau)\right) s\right]\right| \leq\left|\int_{p T}^{t} D F\left(y_{\varepsilon}(\tau), s\right)\right|+\left|\int_{p T}^{t} D\left[F_{0}\left(y_{\varepsilon}(\tau)\right) s\right]\right| \\
\leq h_{1}(t)-h_{1}(p T)+h_{3}(t)-h_{3}(p T) \\
\leq h_{1}(p T+T)-h_{1}(p T)+h_{3}(p T+T)-h_{3}(p T) \leq \alpha+m+l .
\end{gathered}
$$


By combining the previous inequalities, we obtain

$$
\left|\int_{0}^{t} D\left[F\left(y_{\varepsilon}(\tau), s\right)-F_{0}\left(y_{\varepsilon}(\tau)\right) s\right]\right| \leq K
$$

where $K$ is a certain constant. It follows that

$$
\left|x_{\varepsilon}(t)-y_{\varepsilon}(t)\right| \leq \varepsilon \int_{0}^{t}\left|x_{\varepsilon}(s)-y_{\varepsilon}(s)\right| \mathrm{d} h_{1}(s)+\varepsilon(J+K+L \beta) .
$$

Then, since $x_{\varepsilon}$ is a regulated function (we have used Lemma 2.16) and $y_{\varepsilon}$ is a continuous functions, both functions must be bounded in a compact interval and we can apply Gronwall's inequality from Theorem 2.10 to obtain

$$
\left|x_{\varepsilon}(t)-y_{\varepsilon}(t)\right| \leq e^{\varepsilon\left(h_{1}(t)-h_{1}(0)\right)} \varepsilon(J+K+L \beta) .
$$

The proof is concluded after observing that

$$
\begin{gathered}
\varepsilon\left(h_{1}(t)-h_{1}(0)\right) \leq \varepsilon\left(h_{1}(L / \varepsilon)-h_{1}(0)\right) \leq \varepsilon\left(h_{1}(\lceil L /(\varepsilon T)\rceil T)-h_{1}(0)\right) \\
\leq \varepsilon\left\lceil\frac{L}{\varepsilon T}\right\rceil \alpha \leq \varepsilon\left(\frac{L}{\varepsilon T}+1\right) \alpha \leq\left(\frac{L}{T}+\varepsilon_{0}\right) \alpha .
\end{gathered}
$$

\subsubsection{Measure FDEs and functional dynamic equations on time scales}

In this section, we present periodic averaging principles for measure FDEs and for functional dynamic equations on time scales.

We start by presenting a periodic averaging principle for measure FDEs. Its proof follows the same basic ideas we used in the proof of Theorem 7.11. Certain details are inspired by the paper [35] which is devoted to non-periodic averaging. Our result is original and it is contained in [84].

Theorem 7.12. Let $\varepsilon_{0}>0, L>0, X \subset G\left([-r, 0], \mathbb{R}^{n}\right), \tilde{X}=\left\{x:[-r, \infty) \rightarrow \mathbb{R}^{n} ; x_{t} \in\right.$ $X$ for every $t \geq 0\}$. Consider a pair of bounded functions $f: X \times[0, \infty) \rightarrow \mathbb{R}^{n}, g:$ $X \times[0, \infty) \times\left(0, \varepsilon_{0}\right] \rightarrow \mathbb{R}^{n}$ and a nondecreasing function $h:[0, \infty) \rightarrow \mathbb{R}$ such that the following conditions are satisfied:

1. For every $x \in \tilde{X}$ and $\varepsilon \in\left(0, \varepsilon_{0}\right]$, the functions $t \mapsto f\left(x_{t}, t\right)$ and $t \mapsto g\left(x_{t}, t, \varepsilon\right)$ are regulated on $[0, \infty)$. 
2. $f$ is T-periodic in the second variable.

3. There is a constant $\alpha>0$ such that $h(t+T)-h(t)=\alpha$ for every $t \geq 0$.

4. There is a constant $C>0$ such that for $x, y \in X$ and $t \in[0, \infty)$,

$$
|f(x, t)-f(y, t)| \leq C\|x-y\|_{\infty} .
$$

Define

$$
f_{0}(x)=\frac{1}{T} \int_{0}^{T} f(x, s) \mathrm{d} h(s), \quad x \in X .
$$

Let $\phi \in X$. Suppose for every $\varepsilon \in\left(0, \varepsilon_{0}\right]$, the initial value problems

$$
\left\{\begin{array}{l}
x(t)=x(0)+\varepsilon \int_{0}^{t} f\left(x_{s}, s\right) \mathrm{d} h(s)+\varepsilon^{2} \int_{0}^{t} g\left(x_{s}, s, \varepsilon\right) \mathrm{d} h(s) \\
x_{0}=\phi
\end{array}\right.
$$

and

$$
\left\{\begin{array}{l}
y(t)=y(0)+\varepsilon \int_{0}^{t} f_{0}\left(y_{s}\right) \mathrm{d} s \\
y_{0}=\phi
\end{array}\right.
$$

have solutions $x^{\varepsilon}, y^{\varepsilon}:\left[0, \frac{L}{\varepsilon}\right] \rightarrow \mathbb{R}^{n}$ respectively. Then there exists a constant $J>0$ such that

$$
\left|x^{\varepsilon}(t)-y^{\varepsilon}(t)\right| \leq J \varepsilon,
$$

for every $\varepsilon \in\left(0, \varepsilon_{0}\right]$ and $t \in\left[0, \frac{L}{\varepsilon}\right]$.

Proof. There is a constant $M>0$ such that $|f(x, t)| \leq M$ and $|g(x, t, \varepsilon)| \leq M$ for every $x \in X, t \in[0, \infty)$ and $\varepsilon \in\left(0, \varepsilon_{0}\right]$. It follows that

$$
\left|f_{0}(x)\right|=\left|\frac{1}{T} \int_{0}^{T} f(x, s) \mathrm{d} h(s)\right| \leq \frac{M}{T}[h(T)-h(0)]=\frac{M \alpha}{T} .
$$

Thus if $\varepsilon \in\left(0, \varepsilon_{0}\right], s, t \in[0, \infty), s \geq t$, the solution $y^{\varepsilon}$ satisfies

$$
\begin{gathered}
\left|y^{\varepsilon}(s+\theta)-y^{\varepsilon}(t+\theta)\right|=\left|\varepsilon \int_{t+\theta}^{s+\theta} f_{0}\left(y_{\sigma}^{\varepsilon}\right) \mathrm{d} \sigma\right| \leq \frac{\varepsilon M(s-t) \alpha}{T}, \quad \theta \in[-r, 0], \\
\left\|y_{s}^{\varepsilon}-y_{t}^{\varepsilon}\right\|_{\infty}=\sup _{\theta \in[-r, 0]}\left|y^{\varepsilon}(s+\theta)-y^{\varepsilon}(t+\theta)\right| \leq \frac{\varepsilon M(s-t) \alpha}{T} .
\end{gathered}
$$


For every $t \in[0, L / \varepsilon]$, we have

$$
\begin{array}{r}
\left|x^{\varepsilon}(t)-y^{\varepsilon}(t)\right|=\left|\varepsilon \int_{0}^{t} f\left(x_{s}^{\varepsilon}, s\right) \mathrm{d} h(s)+\varepsilon^{2} \int_{0}^{t} g\left(x_{s}^{\varepsilon}, s, \varepsilon\right) \mathrm{d} h(s)-\varepsilon \int_{0}^{t} f_{0}\left(y_{s}^{\varepsilon}\right) \mathrm{d} s\right| \\
\leq \varepsilon\left|\int_{0}^{t}\left(f\left(x_{s}^{\varepsilon}, s\right)-f\left(y_{s}^{\varepsilon}, s\right)\right) \mathrm{d} h(s)\right|+\varepsilon\left|\int_{0}^{t} f\left(y_{s}^{\varepsilon}, s\right) \mathrm{d} h(s)-\int_{0}^{t} f_{0}\left(y_{s}^{\varepsilon}\right) \mathrm{d} s\right| \\
+\varepsilon^{2}\left|\int_{0}^{t} g\left(x_{s}^{\varepsilon}, s, \varepsilon\right) \mathrm{d} h(s)\right| \\
\leq \varepsilon \int_{0}^{t} C\left\|x_{s}^{\varepsilon}-y_{s}^{\varepsilon}\right\|_{\infty} \mathrm{d} h(s)+\varepsilon\left|\int_{0}^{t} f\left(y_{s}^{\varepsilon}, s\right) \mathrm{d} h(s)-\int_{0}^{t} f_{0}\left(y_{s}^{\varepsilon}\right) \mathrm{d} s\right|+\varepsilon^{2} M[h(t)-h(0)] .
\end{array}
$$

At first, we estimate the second term of (7.2.6). Let $p$ be the largest integer such that $p T \leq t$. Then

$$
\begin{gathered}
\left|\int_{0}^{t} f\left(y_{s}^{\varepsilon}, s\right) \mathrm{d} h(s)-\int_{0}^{t} f_{0}\left(y_{s}^{\varepsilon}\right) \mathrm{d} s\right| \leq \\
\leq \sum_{i=1}^{p}\left|\int_{(i-1) T}^{i T}\left(f\left(y_{s}^{\varepsilon}, s\right)-f\left(y_{(i-1) T}^{\varepsilon}, s\right)\right) \mathrm{d} h(s)\right| \\
+\sum_{i=1}^{p}\left|\int_{(i-1) T}^{i T} f\left(y_{(i-1) T}^{\varepsilon}, s\right) \mathrm{d} h(s)-\int_{(i-1) T}^{i T} f_{0}\left(y_{(i-1) T}^{\varepsilon}\right) \mathrm{d} s\right| \\
+\sum_{i=1}^{p}\left|\int_{(i-1) T}^{i T}\left(f_{0}\left(y_{(i-1) T}^{\varepsilon}\right)-f_{0}\left(y_{s}^{\varepsilon}\right)\right) \mathrm{d} s\right|+\left|\int_{p T}^{t} f\left(y_{s}^{\varepsilon}, s\right) \mathrm{d} h(s)-\int_{p T}^{t} f_{0}\left(y_{s}^{\varepsilon}\right) \mathrm{d} s\right| .
\end{gathered}
$$

For every $i \in\{1,2, \ldots, p\}$ and every $s \in[(i-1) T, i T]$, inequality (7.2.5) gives

$$
\left\|y_{s}^{\varepsilon}-y_{(i-1) T}^{\varepsilon}\right\|_{\infty} \leq \frac{M \varepsilon \alpha(s-(i-1) T)}{T} \leq M \varepsilon \alpha
$$

Using this estimate together with the fact that $p T \leq \frac{L}{\varepsilon}$, we obtain

$$
\begin{aligned}
\sum_{i=1}^{p}\left|\int_{(i-1) T}^{i T}\left(f\left(y_{s}^{\varepsilon}, s\right)-f\left(y_{(i-1) T}^{\varepsilon}, s\right)\right) \mathrm{d} h(s)\right| & \leq \sum_{i=1}^{p} C M \varepsilon \alpha(h(i T)-h((i-1) T)) \\
& =C M \varepsilon \alpha^{2} p \leq \frac{C M L \alpha^{2}}{T}
\end{aligned}
$$

For every $y \in \tilde{X}$, we have

$$
\begin{aligned}
\left|f_{0}\left(y_{s}\right)-f_{0}\left(y_{t}\right)\right| & \leq \frac{1}{T}\left|\int_{0}^{T}\left(f\left(y_{s}, \sigma\right)-f\left(y_{t}, \sigma\right)\right) d h(\sigma)\right| \\
& \leq \frac{C}{T}\left\|y_{s}-y_{t}\right\|_{\infty}(h(T)-h(0))=\frac{C}{T}\left\|y_{s}-y_{t}\right\|_{\infty} \alpha .
\end{aligned}
$$


Thus,

$$
\begin{aligned}
& \sum_{i=1}^{p}\left|\int_{(i-1) T}^{i T}\left(f_{0}\left(y_{s}^{\varepsilon}\right)-f_{0}\left(y_{(i-1) T}^{\varepsilon}\right)\right) \mathrm{d} s\right| \leq \sum_{i=1}^{p} \int_{(i-1) T}^{i T}\left|f_{0}\left(y_{s}^{\varepsilon}\right)-f_{0}\left(y_{(i-1) T}^{\varepsilon}\right)\right| \mathrm{d} s \\
\leq & \frac{C}{T} \alpha \sum_{i=1}^{p} \int_{(i-1) T}^{i T}\left\|y_{s}^{\varepsilon}-y_{(i-1) T}^{\varepsilon}\right\|_{\infty} \mathrm{d} s \leq \frac{C}{T} \alpha \sum_{i=1}^{p} \varepsilon M \alpha T=\varepsilon M C \alpha^{2} p \leq \frac{M C L \alpha^{2}}{T} .
\end{aligned}
$$

The fact that $f$ is $T$-periodic in the second variable and the definition of $f_{0}$ imply

$$
\begin{gathered}
\sum_{i=1}^{p}\left|\int_{(i-1) T}^{i T} f\left(y_{(i-1) T}^{\varepsilon}, s\right) \mathrm{d} h(s)-\int_{(i-1) T}^{i T} f_{0}\left(y_{(i-1) T}^{\varepsilon}\right) \mathrm{d} s\right|= \\
=\sum_{i=1}^{p}\left|\int_{0}^{T} f\left(y_{(i-1) T}^{\varepsilon}, s\right) \mathrm{d} h(s)-f_{0}\left(y_{(i-1) T}^{\varepsilon}\right) T\right|=0 .
\end{gathered}
$$

Finally,

$$
\begin{gathered}
\left|\int_{p T}^{t} f\left(y_{s}^{\varepsilon}, s\right) \mathrm{d} h(s)-\int_{p T}^{t} f_{0}\left(y_{s}^{\varepsilon}\right) \mathrm{d} s\right| \leq\left|\int_{p T}^{t} f\left(y_{s}^{\varepsilon}, s\right) \mathrm{d} h(s)\right|+\int_{p T}^{t}\left|f_{0}\left(y_{s}^{\varepsilon}\right)\right| \mathrm{d} s \\
\leq M(h(t)-h(p T))+\frac{M \alpha}{T}(t-p T) \\
\leq M(h((p+1) T)-h(p T))+\frac{M \alpha}{T} T=M \alpha+M \alpha=2 M \alpha .
\end{gathered}
$$

By combination of the previous results, we obtain

$$
\left|\int_{0}^{t} f\left(y_{s}^{\varepsilon}, s\right) \mathrm{d} h(s)-\int_{0}^{t} f_{0}\left(y_{s}^{\varepsilon}\right) \mathrm{d} s\right| \leq \frac{2 M C L \alpha^{2}}{T}+2 M \alpha
$$

Denote the constant on the right-hand side of (7.2.7) by $K$. Returning back to inequality (7.2.6), we see that

$$
\left|x^{\varepsilon}(t)-y^{\varepsilon}(t)\right| \leq \varepsilon \int_{0}^{t} C\left\|x_{s}^{\varepsilon}-y_{s}^{\varepsilon}\right\|_{\infty} \mathrm{d} h(s)+\varepsilon K+\varepsilon^{2} M(h(t)-h(0)) .
$$

Let $\psi(s)=\sup _{\tau \in[0, s]}\left|x^{\varepsilon}(\tau)-y^{\varepsilon}(\tau)\right|$. Then, for every $u \in[0, t]$, we have

$$
\begin{aligned}
\left|x^{\varepsilon}(u)-y^{\varepsilon}(u)\right| & \leq \varepsilon \int_{0}^{u} C \psi(s) \mathrm{d} h(s)+\varepsilon K+\varepsilon^{2} M(h(u)-h(0)) \\
& \leq \varepsilon \int_{0}^{t} C \psi(s) \mathrm{d} h(s)+\varepsilon K+\varepsilon^{2} M(h(t)-h(0)) .
\end{aligned}
$$


Consequently,

$$
\psi(t) \leq \varepsilon \int_{0}^{t} C \psi(s) \mathrm{d} h(s)+\varepsilon K+\varepsilon^{2} M(h(t)-h(0)) .
$$

Next, note that

$$
\begin{aligned}
\varepsilon[h(t)-h(0)] & \leq \varepsilon[h(L / \varepsilon)-h(0)] \leq \varepsilon[h(\lceil L /(\varepsilon T)\rceil T)-h(0)] \\
& \leq \varepsilon\left\lceil\frac{L}{\varepsilon T}\right\rceil \alpha \leq \varepsilon\left(\frac{L}{\varepsilon T}+1\right) \alpha \leq\left(\frac{L}{T}+\varepsilon_{0}\right) \alpha .
\end{aligned}
$$

Thus,

$$
\psi(t) \leq \varepsilon \int_{0}^{t} C \psi(s) \mathrm{d} h(s)+\varepsilon K+\varepsilon M\left(\frac{L}{T}+\varepsilon_{0}\right) \alpha .
$$

Then the Gronwall's inequality from Theorem 2.10 gives

$$
\psi(t) \leq e^{\varepsilon C(h(t)-h(0))}\left(K+M\left(\frac{L}{T}+\varepsilon_{0}\right) \alpha\right) \varepsilon \leq e^{C\left(\frac{L}{T}+\varepsilon_{0}\right) \alpha}\left(K+M\left(\frac{L}{T}+\varepsilon_{0}\right) \alpha\right) \varepsilon
$$

Thus, if we take $J=e^{C\left(\frac{L}{T}+\varepsilon_{0}\right) \alpha}\left(K+M\left(\left(\frac{L}{T}+\varepsilon_{0}\right) \alpha\right)\right)$, we obtain

$$
\left|x^{\varepsilon}(t)-y^{\varepsilon}(t)\right| \leq \psi(t) \leq J \varepsilon,
$$

for every $\varepsilon \in\left(0, \varepsilon_{0}\right]$ and $t \in\left[0, \frac{L}{\varepsilon}\right]$.

In order to be able to speak about periodic functions on time scales, we need the following concept of a periodic time scale.

Definition 7.13. Let $T>0$ be a real number. A time scale $\mathbb{T}$ is called $T$-periodic, if $t \in \mathbb{T}$ implies $t+T \in \mathbb{T}$ and $\mu(t)=\mu(t+T)$.

We now proceed to the periodic averaging theorem for functional dynamic equations on time scales. This is an original result and it is contained in [36].

Theorem 7.14. Assume that $T>0, \mathbb{T}$ is a T-periodic time scale, $t_{0} \in \mathbb{T}, \varepsilon_{0}>0, L>0$, $B \subset \mathbb{R}^{n}$. Consider a pair of bounded functions $f: G([-r, 0], B) \times\left[t_{0}, \infty\right)_{\mathbb{T}} \rightarrow \mathbb{R}^{n}$ and $g: G([-r, 0], B) \times\left[t_{0}, \infty\right)_{\mathbb{T}} \times\left(0, \varepsilon_{0}\right] \rightarrow \mathbb{R}^{n}$ such that the following conditions are satisfied:

1. For every $b>t_{0}$ and $y \in G\left(\left[t_{0}-r, b\right], B\right)$, the function $t \mapsto f\left(y_{t}, t\right)$ is regulated on $\left[t_{0}, b\right]_{\mathbb{T}}$.

2. For every $b>t_{0}$ and $y \in C\left(\left[t_{0}-r, b\right]_{\mathbb{T}}, B\right)$, the function $t \mapsto f\left(y_{t}^{*}, t\right)$ is rd-continuous on $\left[t_{0}, b\right]_{\mathbb{T}}$. 
3. $f$ is Lipschitz-continuous in the first variable.

4. $f$ is T-periodic and rd-continuous in the second variable.

Denote

$$
f_{0}(y)=\frac{1}{T} \int_{t_{0}}^{t_{0}+T} f(y, s) \Delta s, \quad y \in G([-r, 0], B) .
$$

Let $\phi \in C\left(\left[t_{0}-r, t_{0}\right]_{\mathbb{T}}, B\right)$. Suppose, for every $\varepsilon \in\left(0, \varepsilon_{0}\right]$, the functional dynamic equation

$$
\begin{aligned}
x^{\Delta}(t) & =\varepsilon f\left(x_{t}^{*}, t\right)+\varepsilon^{2} g\left(x_{t}^{*}, t, \varepsilon\right), \\
x(t) & =\phi(t), \quad t \in\left[t_{0}-r, t_{0}\right]_{\mathbb{T}}
\end{aligned}
$$

has a solution $x_{\varepsilon}:\left[t_{0}-r, t_{0}+L / \varepsilon\right]_{\mathbb{T}} \rightarrow \mathbb{R}^{n}$, and the functional differential equation

$$
\begin{aligned}
\dot{y} & =f_{0}\left(y_{s}\right), \\
y_{t_{0}} & =\phi^{*}{ }_{t_{0}}
\end{aligned}
$$

has a solution $y_{\varepsilon}:\left[t_{0}-r, t_{0}+L / \varepsilon\right] \rightarrow \mathbb{R}^{n}$. Then there exists a constant $J>0$ such that

$$
\left|x_{\varepsilon}(t)-y_{\varepsilon}(t)\right| \leq J \varepsilon
$$

for every $\varepsilon \in\left(0, \varepsilon_{0}\right]$ and $t \in\left[t_{0}, t_{0}+L / \varepsilon\right]_{\mathbb{T}}$.

Proof. Without loss of generality, we can assume that $t_{0}=0$. Otherwise, consider a shifted problem with the time scale $\widetilde{\mathbb{T}}=\left\{t-t_{0} ; t \in \mathbb{T}\right\}$ and the right-hand side $\widetilde{f}(x, t)=$ $f\left(x, t+t_{0}\right)$.

For every $t \in[0, \infty), y \in G([-r, 0], B)$ and $\varepsilon \in\left(0, \varepsilon_{0}\right]$, let

$$
f^{*}(y, t)=f\left(y, t^{*}\right) \quad \text { and } \quad g^{*}(y, t, \varepsilon)=g\left(y, t^{*}, \varepsilon\right) .
$$

Also, let $h(t)=t^{*}$ for every $t \in[0, \infty)$. It follows directly from the definition of $h$ and the fact that $\mathbb{T}$ is $T$-periodic that

$$
h(t+T)-h(t)=T, \quad t \geq 0 .
$$


By Theorem $4.13, x_{\varepsilon}^{*}$ satisfies

$$
\begin{aligned}
& x_{\varepsilon}^{*}(t)=x_{\varepsilon}^{*}(0)+\varepsilon \int_{0}^{t} f^{*}\left(\left(x_{\varepsilon}^{*}\right)_{s}, s\right) \mathrm{d} h(s)+\varepsilon^{2} \int_{0}^{t} g^{*}\left(\left(x_{\varepsilon}^{*}\right)_{s}, s, \varepsilon\right) \mathrm{d} h(s), \quad t \in[0, L / \varepsilon] \\
& \left(x_{\varepsilon}^{*}\right)_{0}=\phi_{0}^{*} .
\end{aligned}
$$

for every $\varepsilon \in\left(0, \varepsilon_{0}\right]$. From Theorem 4.11, we have

$$
f_{0}(y)=\frac{1}{T} \int_{0}^{T} f(y, s) \Delta s=\frac{1}{T} \int_{0}^{T} f^{*}(y, s) \mathrm{d} h(s), \quad y \in G([-r, 0], B) .
$$

For every $b \in[0, \infty)_{\mathbb{T}}$ and $y \in G([-r, b], B)$, the function $u(t)=f\left(y_{t}, t\right)$ is regulated on $[0, b]_{\mathbb{T}}$. Consequently, there is a sequence of continuous functions $u_{n}:[0, b]_{\mathbb{T}} \rightarrow \mathbb{R}^{n}, n \in \mathbb{N}$, which is uniformly convergent to $u$. It follows that $\left\{u_{n}^{*}\right\}_{n=1}^{\infty}$ is uniformly convergent to $u^{*}$ on $[0, b]$. Using Theorem 4.11 and uniform convergence theorems for the KurzweilHenstock-Stieltjes and $\Delta$-integrals, we obtain

$$
\int_{0}^{b} u(t) \Delta t=\lim _{n \rightarrow \infty} \int_{0}^{b} u_{n}(t) \Delta t=\lim _{n \rightarrow \infty} \int_{0}^{b} u_{n}^{*}(t) \mathrm{d} h(t)=\int_{0}^{b} u^{*}(t) \mathrm{d} h(t)=\int_{0}^{b} f\left(y_{t^{*}}, t^{*}\right) \mathrm{d} h(t) .
$$

Theorem 4.12 implies the existence of $\int_{0}^{b} f^{*}\left(y_{t}, t\right) \mathrm{d} h(t)$.

Since $f^{*}$ and $g^{*}$ satisfy all assumptions of Theorem 7.12 , there exists a constant $J>0$ such that

$$
\left|x_{\varepsilon}^{*}(t)-y_{\varepsilon}(t)\right| \leq J \varepsilon,
$$

for every $\varepsilon \in\left(0, \varepsilon_{0}\right]$ and $t \in[0, L / \varepsilon]$. The proof is finished after observing that $x_{\varepsilon}^{*}(t)=$ $x_{\varepsilon}(t)$ for $t \in[0, L / \varepsilon]_{\mathbb{T}}$.

\subsubsection{Impulsive measure FDEs and impulsive functional dynamic equations on time scales}

In this section, we present periodic averaging principles for measure FDEs and for functional dynamic equations involving impulses.

We start by proving a periodic averaging theorem for measure FDEs with impulses. This is a new result which can be found in [37].

Theorem 7.15. Assume that $\varepsilon_{0}>0, L>0, B \subset \mathbb{R}^{n}, X=G([-r, 0], B), m \in \mathbb{N}$ and $0 \leq t_{1}<t_{2}<\cdots<t_{m}<T$. Consider a pair of bounded functions $f: X \times[0, \infty) \rightarrow \mathbb{R}^{n}$, 
$g: X \times[0, \infty) \times\left(0, \varepsilon_{0}\right] \rightarrow \mathbb{R}^{n}$ and a nondecreasing left continuous function $h:[0, \infty) \rightarrow \mathbb{R}$ which is continuous at $t_{1}, \ldots, t_{m}$. Let $I_{k}: B \rightarrow \mathbb{R}^{n}, k \in\{1,2, \ldots, m\}$ be bounded and Lipschitz continuous functions. For every integer $k>m$, define $t_{k}$ and $I_{k}$ by the recursive formulas $t_{k}=t_{k-m}+T$ and $I_{k}=I_{k-m}$. Suppose the following conditions are satisfied:

1. The integral $\int_{0}^{b} f\left(y_{t}, t\right) \mathrm{d} h(t)$ exists, for every $b>0$ and $y \in G([-r, b], B)$.

2. $f$ is Lipschitz continuous with respect to the first variable.

3. $f$ is T-periodic in the second variable.

4. There is a constant $\alpha>0$ such that $h(t+T)-h(t)=\alpha$ for every $t \geq 0$.

5. The integral

$$
f_{0}(x)=\frac{1}{T} \int_{0}^{T} f(x, s) \mathrm{d} h(s)
$$

exists for every $x \in X$.

Denote

$$
I_{0}(y)=\frac{1}{T} \sum_{k=1}^{m} I_{k}(y), \quad y \in B .
$$

Let $\phi \in X$ and suppose, for every $\varepsilon \in\left(0, \varepsilon_{0}\right]$, the initial value problems

$$
\left\{\begin{array}{l}
x(t)=x(0)+\varepsilon \int_{0}^{t} f\left(x_{s}, s\right) \mathrm{d} h(s)+\varepsilon^{2} \int_{0}^{t} g\left(x_{s}, s, \varepsilon\right) \mathrm{d} h(s)+\varepsilon \sum_{\substack{k \in \mathbb{N}, t_{k}<t}} I_{k}\left(x\left(t_{k}\right)\right), \\
x_{0}=\phi
\end{array}\right.
$$

and

$$
\left\{\begin{array}{l}
y(t)=y(0)+\varepsilon \int_{0}^{t}\left(f_{0}\left(y_{s}\right)+I_{0}(y(s))\right) \mathrm{d} s \\
y_{0}=\phi
\end{array}\right.
$$

have solutions $x^{\varepsilon}, y^{\varepsilon}:[-r, L / \varepsilon] \rightarrow B$ respectively. Then there exists a constant $J>0$ such that

$$
\left|x^{\varepsilon}(t)-y^{\varepsilon}(t)\right| \leq J \varepsilon,
$$

for every $\varepsilon \in\left(0, \varepsilon_{0}\right]$ and $t \in[0, L / \varepsilon]$.

Proof. Define a function $\tilde{h}(t):[0, \infty) \rightarrow \mathbb{R}$ by

$$
\tilde{h}(t)= \begin{cases}h(t), & t \in\left[0, t_{1}\right], \\ h(t)+c_{k}, & t \in\left(t_{k}, t_{k+1}\right] \text { for some } k \in \mathbb{N}\end{cases}
$$


where the sequence $\left\{c_{k}\right\}_{k=1}^{\infty}$ is chosen in such a way that $\Delta^{+} \tilde{h}\left(t_{k}\right)=1$ for every $k \in \mathbb{N}$. Note that $\tilde{h}$ is nondecreasing, left continuous, and there exists a constant $\tilde{\alpha}>0$ such that

$$
\tilde{h}(t+T)-\tilde{h}(t)=\tilde{\alpha}
$$

for every $t \geq 0$.

According to the assumptions, we have

$$
x^{\varepsilon}(t)=x^{\varepsilon}(0)+\int_{0}^{t}\left(\varepsilon f\left(x_{s}^{\varepsilon}, s\right)+\varepsilon^{2} g\left(x_{s}^{\varepsilon}, s, \varepsilon\right)\right) \mathrm{d} h(s)+\sum_{\substack{k \in \mathbb{N}, t_{k}<t}} \varepsilon I_{k}\left(x^{\varepsilon}\left(t_{k}\right)\right),
$$

for every $\varepsilon \in\left(0, \varepsilon_{0}\right]$ and $t \in[0, L / \varepsilon]$. Let

$$
F^{\varepsilon}(y, t)= \begin{cases}\varepsilon f(y, t)+\varepsilon^{2} g(y, t, \varepsilon), & t \notin\left\{t_{1}, t_{2}, \ldots\right\}, \\ \varepsilon I_{k}(y(0)), & t=t_{k} \text { for some } k \in \mathbb{N},\end{cases}
$$

for every $y \in X$ and $t \geq 0$. By Theorem 4.16, we obtain

$$
x^{\varepsilon}(t)=x^{\varepsilon}(0)+\int_{0}^{t} F^{\varepsilon}\left(x_{s}^{\varepsilon}, s\right) \mathrm{d} \tilde{h}(s)
$$

for every $\varepsilon \in\left(0, \varepsilon_{0}\right]$ and $t \in[0, L / \varepsilon]$. For every $y \in X$ and $t \geq 0$, we have

$$
F^{\varepsilon}(y, t)=\varepsilon \tilde{f}(y, t)+\varepsilon^{2} \tilde{g}(y, t, \varepsilon)
$$

where

$$
\tilde{f}(y, t)= \begin{cases}f(y, t), & t \notin\left\{t_{1}, t_{2}, \ldots\right\} \\ I_{k}(y(0)), & t=t_{k} \text { for some } k \in \mathbb{N}\end{cases}
$$

and

$$
\tilde{g}(y, t, \varepsilon)= \begin{cases}g(y, t, \varepsilon), & t \notin\left\{t_{1}, t_{2}, \ldots\right\}, \\ 0, & t=t_{k} \text { for some } k \in \mathbb{N} .\end{cases}
$$

It follows from (7.2.8) and (7.2.9) that for every $\varepsilon \in\left(0, \varepsilon_{0}\right]$, the function $x^{\varepsilon}:[-r, L / \varepsilon] \rightarrow B$ is a solution of the initial value problem

$$
\begin{aligned}
x(t) & =x(0)+\varepsilon \int_{0}^{t} \tilde{f}\left(x_{s}, s\right) \mathrm{d} \tilde{h}(s)+\varepsilon^{2} \int_{0}^{t} \tilde{g}\left(x_{s}, s, \varepsilon\right) \mathrm{d} \tilde{h}(s), \\
x_{0} & =\phi .
\end{aligned}
$$


The function $\tilde{f}$ is Lipschitz continuous with respect to the first variable and $T$-periodic in the second variable. Using Lemma 4.15, we have

$$
\begin{gathered}
\int_{0}^{T} \tilde{f}(x, s) \mathrm{d} \tilde{h}(s)=\int_{0}^{T} f(x, s) \mathrm{d} h(s)+\sum_{k=1}^{m} \tilde{f}\left(x, t_{k}\right) \Delta^{+} \tilde{h}\left(t_{k}\right) \\
=\int_{0}^{T} f(x, s) \mathrm{d} h(s)+\sum_{k=1}^{m} I_{k}(x(0))
\end{gathered}
$$

for every $x \in X$. Consequently, the function

$$
\tilde{f}_{0}(x)=\frac{1}{T} \int_{0}^{T} \tilde{f}(x, s) \mathrm{d} \tilde{h}(s), \quad x \in X
$$

satisfies

$$
\tilde{f}_{0}(x)=\frac{1}{T} \int_{0}^{T} f(x, s) \mathrm{d} h(s)+\frac{1}{T} \sum_{k=1}^{m} I_{k}(x(0))=f_{0}(x)+I_{0}(x(0)), \quad x \in X .
$$

By Theorem 7.12, there exists a constant $J>0$ such that

$$
\left|x^{\varepsilon}(t)-y^{\varepsilon}(t)\right| \leq J \varepsilon,
$$

for every $\varepsilon \in\left(0, \varepsilon_{0}\right]$ and $t \in[0, L / \varepsilon]$ and the proof is complete.

Finally, we present a periodic averaging theorem for impulsive functional dynamic equations on time scales. Such result is new and can be found in [36].

Theorem 7.16. Assume that $\mathbb{T}$ is a $T$-periodic time scale, $\left[t_{0}-r, t_{0}+\sigma\right]_{\mathbb{T}}$ a time scale interval, $t_{0} \in \mathbb{T}, \varepsilon_{0}>0, L>0, B \subset \mathbb{R}^{n}, X=G([-r, 0], B), m \in \mathbb{N}, t_{1}, \ldots, t_{m} \in \mathbb{T}$ are right-dense points such that $t_{0} \leq t_{1}<t_{2}<\cdots<t_{m}<t_{0}+T$. Let $I_{k}: B \rightarrow \mathbb{R}^{n}$, $k \in\{1,2, \ldots, m\}$ be bounded and Lipschitz continuous functions. For every integer $k>m$, define $t_{k}$ and $I_{k}$ by the recursive formulas $t_{k}=t_{k-m}+T$ and $I_{k}=I_{k-m}$. Consider a pair of bounded functions $f: X \times\left[t_{0}, \infty\right)_{\mathbb{T}} \rightarrow \mathbb{R}^{n}, g: X \times\left[t_{0}, \infty\right)_{\mathbb{T}} \times\left(0, \varepsilon_{0}\right] \rightarrow \mathbb{R}^{n}$ such that the following conditions are satisfied:

1. The integral $\int_{0}^{b} f\left(y_{t}, t\right) \Delta t$ exists, for every $b>0$ and $y \in G([-r, b], B)$.

2. $f$ is Lipschitz continuous with respect to the first variable.

3. $f$ is T-periodic in the second variable. 
4. The integral

$$
f_{0}(x)=\frac{1}{T} \int_{t_{0}}^{t_{0}+T} f(x, s) \Delta s
$$

exists for every $x \in X$.

\section{Denote}

$$
I_{0}(y)=\frac{1}{T} \sum_{k=1}^{m} I_{k}(y), \quad y \in B
$$

Let $\phi \in G\left(\left[t_{0}-r, t_{0}\right]_{\mathbb{T}}, B\right)$ and suppose for every $\varepsilon \in\left(0, \varepsilon_{0}\right]$, the initial value problems

$x(t)=x\left(t_{0}\right)+\varepsilon \int_{t_{0}}^{t} f\left(x_{s}^{*}, s\right) \Delta s+\varepsilon^{2} \int_{t_{0}}^{t} g\left(x_{s}^{*}, s, \varepsilon\right) \Delta s+\varepsilon \sum_{\substack{k \in \mathbb{N}, t_{k}<t}} I_{k}\left(y\left(t_{k}\right)\right), \quad t \in\left[t_{0}, t_{0}+L / \varepsilon\right]_{\mathbb{T}}$, $x(t)=\phi(t), \quad t \in\left[t_{0}-r, t_{0}\right]_{\mathbb{T}}$,

and

$$
\begin{aligned}
y(t) & =y\left(t_{0}\right)+\varepsilon \int_{t_{0}}^{t}\left(f_{0}\left(y_{s}\right)+I_{0}(y(s))\right) \mathrm{d} s, \\
y_{t_{0}} & =\phi_{t_{0}}^{*}
\end{aligned}
$$

have solutions $x^{\varepsilon}:\left[t_{0}-r, t_{0}+L / \varepsilon\right]_{\mathbb{T}} \rightarrow B$ and $y^{\varepsilon}:\left[t_{0}-r, t_{0}+L / \varepsilon\right] \rightarrow B$ respectively. Then there exists a constant $J>0$ such that

$$
\left|x^{\varepsilon}(t)-y^{\varepsilon}(t)\right| \leq J \varepsilon,
$$

for every $\varepsilon \in\left(0, \varepsilon_{0}\right]$ and $t \in\left[t_{0}, t_{0}+L / \varepsilon\right]_{\mathbb{T}}$.

Proof. Without loss of generality, we can assume that $t_{0}=0$. Otherwise, consider a shifted problem with time scale $\widetilde{\mathbb{T}}=\left\{t-t_{0} ; t \in \mathbb{T}\right\}$ and functions $\widetilde{f}(x, t)=f\left(x, t+t_{0}\right)$ and $\widetilde{g}(x, t, \varepsilon)=g\left(x, t+t_{0}, \varepsilon\right)$.

For every $t \in\left[t_{0}, \infty\right), x \in X$ and $\varepsilon \in\left(0, \varepsilon_{0}\right]$, let

$$
f^{*}(x, t)=f\left(x, t^{*}\right) \quad \text { and } \quad g^{*}(x, t, \varepsilon)=g\left(x, t^{*}, \varepsilon\right)
$$

Also, let $h(t)=t^{*}$ for every $t \in\left[t_{0}, \infty\right)$. Since $\mathbb{T}$ is $T$-periodic, it follows that

$$
h(t+T)-h(t)=T, \quad t \geq 0 .
$$


From Theorem 4.11, we obtain

$$
f_{0}(x)=\frac{1}{T} \int_{0}^{T} f(x, s) \Delta s=\frac{1}{T} \int_{0}^{T} f^{*}(x, s) \mathrm{d} h(s)
$$

for every $x \in X$.

For every $b>0$ and $y \in G([-r, b], B)$, the integral $\int_{0}^{b} f\left(y_{t}, t\right) \Delta t$ exists. Then, Theorems 4.11 and 4.12 imply

$$
\int_{0}^{b} f\left(y_{t}, t\right) \Delta t=\int_{0}^{b} f\left(y_{t^{*}}, t^{*}\right) \mathrm{d} h(t)=\int_{0}^{b} f\left(y_{t}, t^{*}\right) \mathrm{d} h(t)=\int_{0}^{b} f^{*}\left(y_{t}, t\right) \mathrm{d} h(t)
$$

that is, the last integral exists as well.

It follows from Theorem 4.23 that for every $\varepsilon \in\left(0, \varepsilon_{0}\right]$ and $t \in[0, L / \varepsilon]$, we have

$$
\begin{aligned}
\left(x^{\varepsilon}\right)^{*}(t) & =\left(x^{\varepsilon}\right)^{*}(0)+\varepsilon \int_{0}^{t} f^{*}\left(\left(x^{\varepsilon}\right)_{s}^{*}, s\right) \mathrm{d} h(s)+\varepsilon^{2} \int_{0}^{t} g^{*}\left(\left(x^{\varepsilon}\right)_{s}^{*}, s, \varepsilon\right) \mathrm{d} h(s)+\varepsilon \sum_{\substack{k \in \mathbb{N}, t_{k}<t}} I_{k}\left(\left(x^{\varepsilon}\right)^{*}\left(t_{k}\right)\right), \\
\left(x^{\varepsilon}\right)_{0}^{*} & =\phi_{0}^{*} .
\end{aligned}
$$

Finally, by Theorem 7.15 , there exists a constant $J>0$ such that

$$
\left|\left(x^{\varepsilon}\right)^{*}(t)-y^{\varepsilon}(t)\right| \leq J \varepsilon
$$

for every $\varepsilon \in\left(0, \varepsilon_{0}\right]$ and $t \in[0, L / \varepsilon]$. We conclude the proof by observing that $\left(x^{\varepsilon}\right)^{*}(t)=$ $x^{\varepsilon}(t)$ for $t \in[0, L / \varepsilon]_{\mathbb{T}}$.

\subsection{Examples}

In this section, we present some examples to illustrate the application of averaging principles.

Example 7.17. We consider the original equation given by

$$
\left\{\begin{array}{l}
\dot{x}(t)=4 \varepsilon\left(\cos ^{2} t\right) x(t) \\
x(0)=1
\end{array}\right.
$$

Then, using the definition of $f_{0}$, we obtain the following averaged equation: 


\section{Averaged equation}

$$
\left\{\begin{array}{l}
\dot{y}(t)=2 \varepsilon y(t) \\
y(0)=1
\end{array}\right.
$$

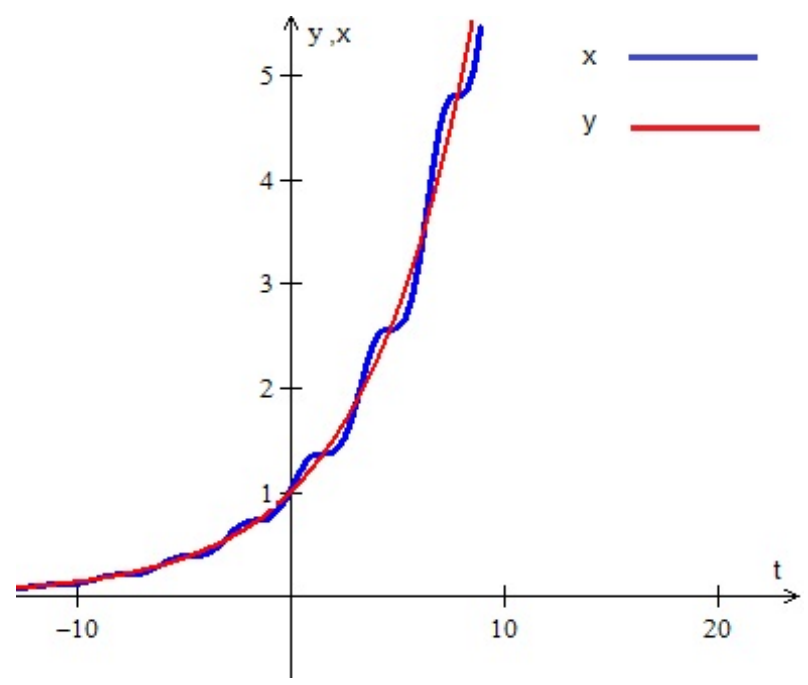

In the graph above, the blue line represents the solution of original equation and the red line represents the solution of averaged equation. We point out that the solution of original equation and the solution of averaged equation are close to each other. Here, we consider $\varepsilon=0.1$.

Example 7.18. Now, we consider the following example of non-periodic averaging.

Let the original equation be given by

$$
\left\{\begin{array}{l}
\dot{x}(t)=\varepsilon\left(e^{-t}\right) x(t) \\
x(0)=1
\end{array}\right.
$$

and the averaged equation be given by

$$
\left\{\begin{array}{l}
\dot{y}(t)=0 \\
y(0)=1
\end{array}\right.
$$

We consider $\varepsilon=0.5$ to plot the graphs above. The blue line represents the solution of original equation and the red line represents the solution of averaged equation.

Example 7.19. Consider the non-autonomous delay differential equation given by

$$
\dot{x}(t)=\varepsilon\left(-4 \cos ^{2}(t) x(t-r)+x(t)\right),
$$




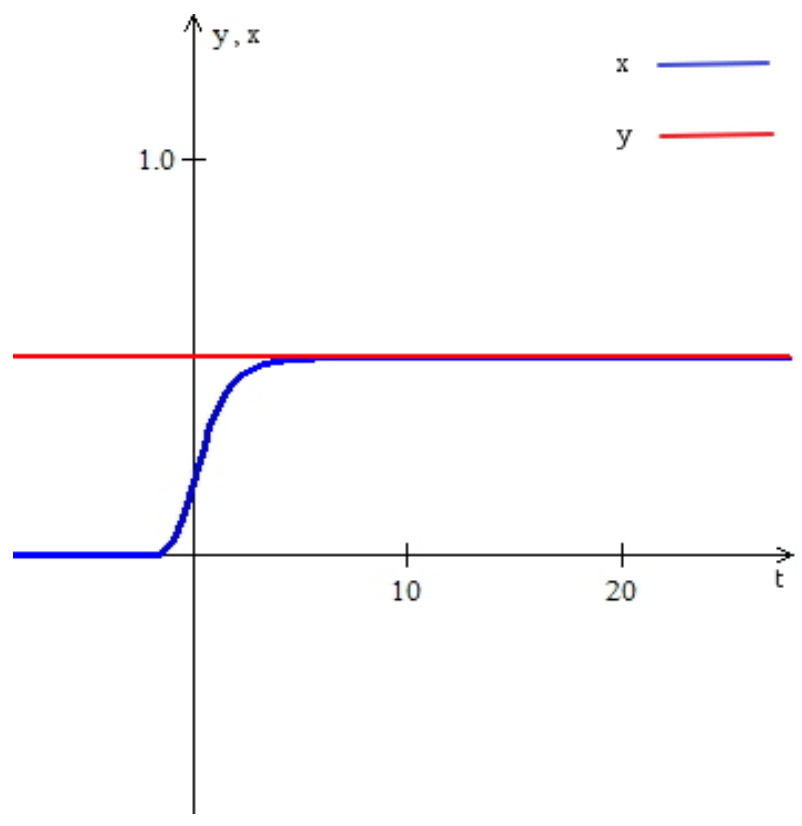

where $x(t)=1$ for $t \in[-r, 0]$.

\section{Averaged equation}

$$
\dot{z}(t)=\varepsilon(-2 z(t-r)+z(t)),
$$

where $z(t)=1$ for $t \in[-r, 0]$.

In the graph below, we consider $\varepsilon=0.1$ and $r=5$.

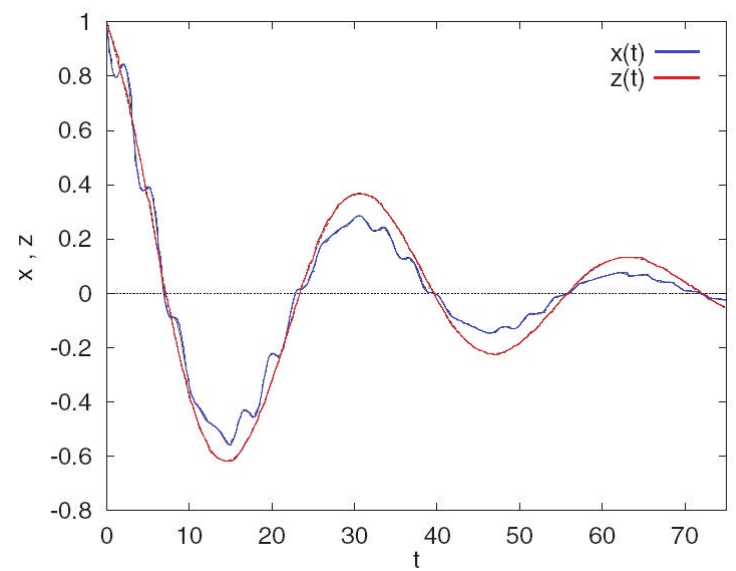





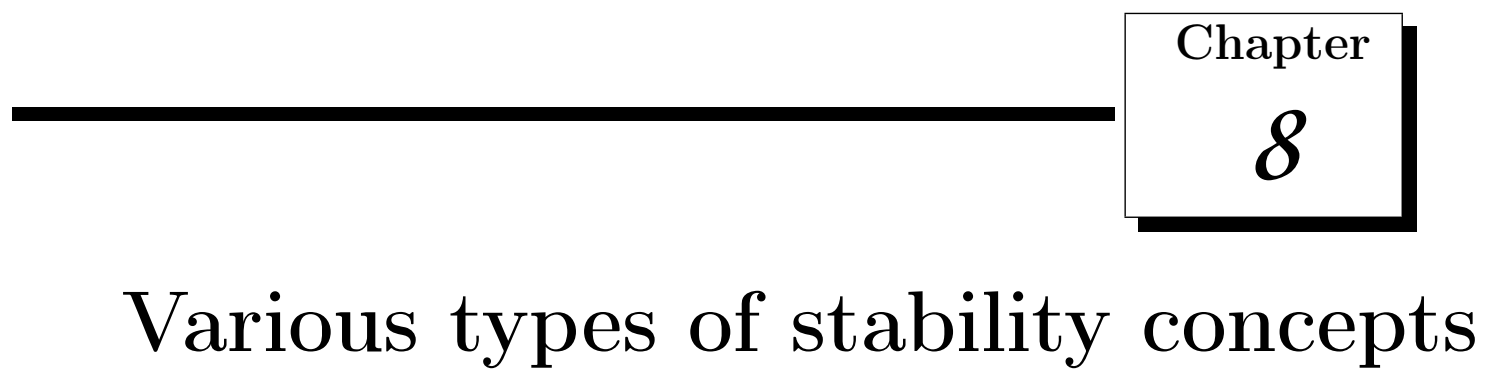

In this chapter, we present new concepts of stability of the trivial solution of various types of equations. This chapter is divided into 6 sections. In the first one, we present new concepts of stability of the trivial solution of measure FDEs. In the second section, we specialize the relation between measure FDEs and generalized ODEs to our purposes in the sequel. The third section is devoted to new concepts of stability of the trivial solution of generalized ODEs. We also present a result which follows from the definitions presented in this section. In the fourth section, we present some relations between the stability concepts for measure FDEs and the corresponding ones for generalized ODEs, using the correspondence between these equations presented in Chapter 4. Section 5 is devoted to new concepts of stability of the trivial solution of impulsive measure FDEs. Also, we relate them with the corresponding ones for measure FDEs and, as a consequence, for generalized ODEs. Finally, in the last section, we introduce new concepts of stability of the trivial solution of impulsive functional dynamic equations on time scales and we relate them to the corresponding ones for other types of equations, using the correspondence between equations presented in our work.

All the results presented in this chapter are new and they are contained in [38].

\subsection{Stability for measure FDEs}

Consider $O \subset G\left(\left[t_{0}-r,+\infty\right), \mathbb{R}^{n}\right)$ with the prolongation property and $P=\left\{y_{t} ; y \in\right.$ $\left.O, t \in\left[t_{0}, \infty\right)\right\} \subset G\left([-r, 0], \mathbb{R}^{n}\right)$. Here, $G\left(\left[t_{0}-r,+\infty\right), \mathbb{R}^{n}\right)$ denotes the set of all bounded 
regulated functions from $\left[t_{0}-r, \infty\right)$ to $\mathbb{R}^{n}$.

In this section, we start by introducing new concepts of stability for measure FDEs. We consider the following equation

$$
D y=f\left(y_{t}, t\right) D g,
$$

where $y_{t}$ is given by the formula $y_{t}(\theta)=y(t+\theta), \theta \in[-r, 0], r>0$, and $D y, D g$ are the distributional derivatives, in the sense of distributions of L. Schwartz, of $y$ and $g$, and $f$ is a function from $P \times\left[t_{0}, \infty\right)$ to $\mathbb{R}^{n}$. The integral form of (8.1.1) is given by

$$
y(t)=y\left(t_{0}\right)+\int_{t_{0}}^{t} f\left(y_{s}, s\right) \mathrm{d} g(s), t \geq t_{0}
$$

where we consider the integral on the right-hand side of (8.1.2) in the sense of KurzweilHenstock-Stieltjes integral taken with respect to a nondecreasing function $g:\left[t_{0}, \infty\right) \rightarrow \mathbb{R}$.

We assume that the function $f: P \times\left[t_{0},+\infty\right) \rightarrow \mathbb{R}^{n}$ satisfies the following conditions:

$\left(H_{1}\right)$ The integral $\int_{t_{0}}^{t} f\left(y_{s}, s\right) \mathrm{d} g(s)$ exists for every $y \in O$ and every $t \in\left[t_{0}, \infty\right)$.

$\left(H_{2}\right)$ There exists a constant $M>0$ such that the inequality

$$
\left|\int_{s_{1}}^{s_{2}} f\left(y_{s}, s\right) \mathrm{d} g(s)\right| \leq M\left[g\left(s_{2}\right)-g\left(s_{1}\right)\right]
$$

holds for every $y \in O$ and every $s_{2}, s_{1} \in\left[t_{0}, \infty\right)$.

$\left(H_{3}\right)$ There exists a constant $L>0$ such that the inequality

$$
\left|\int_{s_{1}}^{s_{2}}\left[f\left(y_{s}, s\right)-f\left(z_{s}, s\right)\right] \mathrm{d} g(s)\right| \leq L \int_{s_{1}}^{s_{2}}\left\|y_{s}-z_{s}\right\|_{\infty} \mathrm{d} g(s)
$$

holds for every $y, z \in O$ and every $s_{1}, s_{2} \in\left[t_{0}, \infty\right)$.

We assume that $f(0, t)=0$ for every $t \in\left[t_{0}, \infty\right)$ so that $y \equiv 0$ is a solution of (8.1.1).

We recall the classical definitions of Lyapunov stability, uniform (Lyapunov) stability and uniform asymptotic stability of the trivial solution of (8.1.1). The reader may want to consult [57], for instance.

Definition 8.1. The trivial solution of system (8.1.1) is called Lyapunov stable, if for every $\varepsilon>0$ and $\gamma \in \mathbb{R}$, there exists $\delta=\delta(\varepsilon, \gamma)>0$ such that if $\phi \in P$ and $\bar{y}:[\gamma, v] \rightarrow \mathbb{R}^{n}$, 
with $[\gamma, v] \subset\left[t_{0},+\infty\right)$ and $[\gamma, v] \ni t_{0}$, is a solution of (8.1.1) such that $\bar{y}_{\gamma}=\phi$ and

$$
\|\phi\|_{\infty}<\delta
$$

then

$$
\left\|\bar{y}_{t}(\gamma, \phi)\right\|_{\infty}<\varepsilon, \quad t \in[\gamma, v]
$$

Definition 8.2. The trivial solution of system (8.1.1) is called uniformly stable, if the number $\delta$ in Definition 8.1 is independent of $\gamma$.

Definition 8.3. The solution $y \equiv 0$ of (8.1.1) is called uniformly asymptotically stable, if it is uniformly stable and if there exists $\delta_{0}>0$ such that for every $\varepsilon>0$, there exists $T=T\left(\varepsilon, \delta_{0}\right) \geq 0$ such that if $\phi \in P$ and $\bar{y}:[\gamma, v] \rightarrow \mathbb{R}^{n}$, with $[\gamma, v] \subset\left[t_{0},+\infty\right)$, is a solution of (8.1.1) such that $\bar{y}_{\gamma}=\phi$ and

$$
\|\phi\|_{\infty}<\delta_{0}
$$

then

$$
\left\|\bar{y}_{t}(\gamma, \phi)\right\|_{\infty}<\varepsilon, \quad t \in[\gamma, v] \cap[\gamma+T,+\infty) .
$$

Now, we consider the perturbed measure FDE given by

$$
D y=f\left(y_{t}, t\right) D g+p(t) D u, \quad t \geq t_{0}
$$

whose integral form is given by

$$
y(t)=y\left(t_{0}\right)+\int_{t_{0}}^{t} f\left(y_{s}, s\right) \mathrm{d} g(s)+\int_{t_{0}}^{t} p(s) \mathrm{d} u(s), \quad t \geq t_{0}
$$

where the integrals are in the sense of Kurzweil-Henstock-Stieltjes. We consider that the functions $g, u:\left[t_{0},+\infty\right) \rightarrow \mathbb{R}$ are nondecreasing and left-continuous and the conditions $\left(H_{1}\right),\left(H_{2}\right)$ and $\left(H_{3}\right)$ are satisfied.

Now, we assume that the function $p:\left[t_{0}, \infty\right) \rightarrow \mathbb{R}^{n}$ satisfies the following conditions:

$\left(H_{4}\right)$ The integral $\int_{t_{0}}^{t} p(s) \mathrm{d} u(s)$ exists for every $t \in\left[t_{0}, \infty\right)$;

$\left(H_{5}\right)$ There exists a constant $K>0$ such that

$$
\left|\int_{s_{1}}^{s_{2}} p(s) \mathrm{d} u(s)\right| \leq K\left[u\left(s_{2}\right)-u\left(s_{1}\right)\right]
$$


for all $s_{1}, s_{2} \in\left[t_{0}, \infty\right)$.

Definition 8.4. The solution $y \equiv 0$ of (8.1.1) is said to be integrally stable, if for every $\varepsilon>0$, there is $\delta=\delta(\varepsilon)>0$ such that if $\psi \in P$ with $\|\psi\|_{\infty}<\delta$ and

$$
\sup _{t \in[\gamma, v]}\left|\int_{\gamma}^{t} p(s) \mathrm{d} u(s)\right|<\delta,
$$

where $t_{0} \leq \gamma \leq v<+\infty$, then

$$
\left|\bar{y}_{t}(\gamma, \psi)\right|<\varepsilon, \text { for every } t \in[\gamma, v] \text {, }
$$

where $\bar{y}(t ; \gamma, \psi)$ is a solution of the perturbed equation (8.1.3) with $y_{\gamma}=\psi$.

Definition 8.5. The solution $y \equiv 0$ of (8.1.1) is called integrally attracting, if there is $\widetilde{\delta}>0$ and for every $\varepsilon>0$, there exist $T=T(\varepsilon) \geq 0$ and $\rho=\rho(\varepsilon)>0$ such that if

$$
\|\psi\|_{\infty}<\widetilde{\delta} \quad \text { and } \quad \sup _{t \in[\gamma, v]}\left|\int_{\gamma}^{t} p(s) \mathrm{d} u(s)\right|<\rho,
$$

where $t_{0} \leq \gamma \leq v<+\infty$, then

$$
\left|\bar{y}_{t}(\gamma, \psi)\right|<\varepsilon \text { for all } t \geq \gamma+T, t \in[\gamma, v]
$$

where $\bar{y}(t ; \gamma, \psi)$ is a solution of equation (8.1.3) with $y_{\gamma}=\psi$.

Definition 8.6. The solution $y \equiv 0$ of (8.1.1) is called integrally asymptotically stable, if it is integrally stable and integrally attracting.

\subsection{Measure FDEs regarded as generalized ODEs}

In this section, we recall the correspondence between generalized ODEs and measure FDEs presented in Chapter 6 and we specialize this correspondence for measure FDEs with perturbations.

Suppose the function $f: P \times\left[t_{0},+\infty\right) \rightarrow \mathbb{R}^{n}$ satisfies conditions $\left(H_{1}\right),\left(H_{2}\right)$ and $\left(H_{3}\right)$ and $p:\left[t_{0},+\infty\right) \rightarrow \mathbb{R}^{n}$ satisfies conditions $\left(H_{4}\right)$ and $\left(H_{5}\right)$. Moreover, assume that the functions $u, g:\left[t_{0},+\infty\right) \rightarrow \mathbb{R}$ are nondecreasing and left-continuous. 
For $y \in O$ and $t \in\left[t_{0},+\infty\right)$, define the following function

$$
F(y, t)(\vartheta)=\left\{\begin{array}{l}
0, \quad t_{0}-r \leq \vartheta \leq t_{0}, \\
\int_{t_{0}}^{\vartheta} f\left(y_{s}, s\right) \mathrm{d} g(s), \quad t_{0} \leq \vartheta \leq t<+\infty \\
\int_{t_{0}}^{t} f\left(y_{s}, s\right) \mathrm{d} g(s), \quad t \leq \vartheta<+\infty
\end{array}\right.
$$

and for $t \in\left[t_{0},+\infty\right)$, define the following function

$$
P(t)(\vartheta)=\left\{\begin{array}{l}
0, \quad t_{0}-r \leq \vartheta \leq t_{0} \\
\int_{t_{0}}^{\vartheta} p(s) \mathrm{d} u(s), \quad t_{0} \leq \vartheta \leq t<+\infty \\
\int_{t_{0}}^{t} p(s) \mathrm{d} u(s), \quad t \leq \vartheta<+\infty .
\end{array}\right.
$$

Then,

$$
G(y, t)=F(y, t)+P(t)
$$

defines an element $G(y, t)$ of $G\left(\left[t_{0}-r,+\infty\right), \mathbb{R}^{n}\right)$ and $G(y, t)(\vartheta) \in \mathbb{R}^{n}$ is the value of $G(y, t)$ at a point $\vartheta \in\left[t_{0}-r,+\infty\right)$, that is,

$$
G: O \times\left[t_{0},+\infty\right) \rightarrow G\left(\left[t_{0}-r,+\infty\right), \mathbb{R}^{n}\right)
$$

Consider the following generalized ODE

$$
\frac{d x}{d \tau}=D G(x, t)
$$

where the function $G$ is given by (8.2.3).

Let $h_{1}, h_{2}:\left[t_{0},+\infty\right) \rightarrow \mathbb{R}$ be defined by

$$
h_{1}(t)=(M+L)\left[g(t)-g\left(t_{0}\right)\right] \text { and } h_{2}(t)=K\left[u(t)-u\left(t_{0}\right)\right], \quad t \in\left[t_{0},+\infty\right) \text {. }
$$

Then, let $h:\left[t_{0},+\infty\right) \rightarrow \mathbb{R}$ be defined by

$$
h(t)=h_{1}(t)+h_{2}(t), \quad t \in\left[t_{0},+\infty\right) .
$$

By the definition of $h$, it is clear that $h$ is left-continuous and nondecreasing. 
If the function $f: P \times\left[t_{0}, \infty\right) \rightarrow \mathbb{R}^{n}$ satisfies conditions $\left(H_{1}\right),\left(H_{2}\right)$ and $\left(H_{3}\right)$ and $p:\left[t_{0}, \infty\right) \rightarrow \mathbb{R}^{n}$ satisfies conditions $\left(H_{4}\right)$ and $\left(H_{5}\right)$, it is not difficult to prove that the function $G$ given by (8.2.3) belongs to the class $\mathcal{F}(\Omega, h)$, where $\Omega=O \times\left[t_{0},+\infty\right)$. For more details of this fact, see [38].

Finally, we state the correspondence between generalized ODEs and measure functional differential equations with perturbations. We omit the proof, since it is similar to the proofs of Theorems 4.5 and 4.6.

Theorem 8.7. The following assertions hold:

(i) Let $O \subset G\left(\left[t_{0}-r, t_{0}+\sigma\right], \mathbb{R}^{n}\right)$ with the prolongation property, $S=\left\{x_{t} ; x \in O, t \in\right.$ $\left.\left[t_{0}, t_{0}+\sigma\right]\right\}, \phi \in S$. Assume that $g:\left[t_{0}, t_{0}+\sigma\right] \rightarrow \mathbb{R}$ and $u:\left[t_{0}, t_{0}+\sigma\right] \rightarrow \mathbb{R}$ are nondecreasing and left-continuous functions, $f: S \times\left[t_{0}, t_{0}+\sigma\right] \rightarrow \mathbb{R}^{n}$ satisfies conditions $\left(H_{1}\right),\left(H_{2}\right),\left(H_{3}\right)$ and $p:\left[t_{0}, t_{0}+\sigma\right] \rightarrow \mathbb{R}^{n}$ satisfies conditions $\left(H_{4}\right)$ and $\left(H_{5}\right)$. Let $y:\left[t_{0}-r, t_{0}+\sigma\right] \rightarrow O$ be a solution of the measure functional differential equation

$$
D y=f\left(y_{t}, t\right) D g+p(t) D u, \quad t \in\left[t_{0}, t_{0}+\sigma\right]
$$

For every $t \in\left[t_{0}-r, t_{0}+\sigma\right]$, let

$$
x(t)(\vartheta)= \begin{cases}y(\vartheta), & \vartheta \in\left[t_{0}-r, t\right] \\ y(t), & \vartheta \in\left[t, t_{0}+\sigma\right] .\end{cases}
$$

Then the function $x:\left[t_{0}, t_{0}+\sigma\right] \rightarrow O$ is a solution of the generalized ordinary differential equation

$$
\frac{d x}{d \tau}=D G(x, t)
$$

with $G$ given by (8.2.3) and initial condition

$$
x\left(t_{0}\right)(\vartheta)=\left\{\begin{array}{l}
\phi\left(\vartheta-t_{0}\right), \text { for } \vartheta \in\left[t_{0}-r, t_{0}\right], \\
x\left(t_{0}\right)\left(t_{0}\right), \text { for } t_{0} \leq \vartheta<t_{0}+\sigma .
\end{array}\right.
$$

(ii) Reciprocally, let $G$ be given by (8.2.3). If $x:\left[t_{0}, t_{0}+\sigma\right] \rightarrow O$ is a solution of the generalized ordinary differential equation

$$
\frac{d x}{d \tau}=D G(x, t)
$$


with initial condition

$$
x\left(t_{0}\right)(\vartheta)=\left\{\begin{array}{l}
\phi\left(\vartheta-t_{0}\right), t_{0}-r \leq \vartheta \leq t_{0}, \\
x\left(t_{0}\right)\left(t_{0}\right), t_{0} \leq \vartheta<t_{0}+\sigma .
\end{array}\right.
$$

Then the function $y:\left[t_{0}-r, t_{0}+\sigma\right] \rightarrow O$ defined by

$$
y(\vartheta)=\left\{\begin{array}{l}
x\left(t_{0}\right)(\vartheta), t_{0}-r \leq \vartheta \leq t_{0} \\
x(\vartheta)(\vartheta), \quad t_{0} \leq \vartheta<t_{0}+\sigma
\end{array}\right.
$$

is a solution of the measure functional differential equation

$$
D y=f\left(y_{t}, t\right) D g+p(t) D u, \quad t \in\left[t_{0}, t_{0}+\sigma\right]
$$

\subsection{Stability for generalized ODEs}

Let $X$ be a Banach space and $B_{c}=\{y \in X ;\|y\|<c\}$ with $c>0$. Define $\Omega=$ $B_{c} \times\left[t_{0}, \infty\right)$. Suppose $F: \Omega \rightarrow X$ belongs to $\mathcal{F}\left(\Omega, h_{1}\right)$, where $h_{1}:\left[t_{0}, \infty\right) \rightarrow \mathbb{R}$ is a nondecreasing and left-continuous function, and satisfies $F(0, t)-F(0, s)=0$, for $t, s \in\left[t_{0},+\infty\right)$. Then, for every $[\gamma, v] \subset\left[t_{0},+\infty\right)$, we have

$$
\int_{\gamma}^{v} D F(0, t)=F(0, v)-F(0, \gamma)=0
$$

which implies that $x \equiv 0$ is a solution on $\left[t_{0}, \infty\right)$ of the generalized ODE

$$
\frac{d x}{d \tau}=D F(x, t)
$$

Recall that if $\bar{x}:[\gamma, v] \rightarrow X$ is a solution of (8.3.1), then the following assertions hold:

(a) $\bar{x}$ is of bounded variation on $[\gamma, v]$;

(b) $\bar{x}(s)=\bar{x}(\gamma)+\int_{\gamma}^{s} D F(\bar{x}(\tau), t)$, for $s \in[\gamma, v]$, by definition.

Now, we present some new concepts concerning stability of the trivial solution of generalized ODEs.

Definition 8.8. The trivial solution $x \equiv 0$ of (8.3.1) is called regularly stable, if for every $\varepsilon>0$, there exists $\delta=\delta(\varepsilon)>0$ such that if $\bar{x}:[\gamma, v] \rightarrow B_{c}$, with $t_{0} \leq \gamma<v<+\infty$, is a 
regulated function which satisfies

$$
\|\bar{x}(\gamma)\|<\delta \quad \text { and } \quad \sup _{s \in[\gamma, v]}\left\|\bar{x}(s)-\bar{x}(\gamma)-\int_{\gamma}^{s} D F(\bar{x}(\tau), t)\right\|<\delta,
$$

then

$$
\|\bar{x}(t)\|<\varepsilon, \quad t \in[\gamma, v]
$$

Definition 8.9. The trivial solution $x \equiv 0$ of (8.3.1) is called regularly attracting if there exists $\delta_{0}>0$ and for every $\varepsilon>0$, there exist $T=T(\varepsilon) \geq 0$ and $\rho=\rho(\varepsilon)>0$ such that if $\bar{x}:[\gamma, v] \rightarrow B_{c}$, with $t_{0} \leq \gamma<v<+\infty$, is a regulated function satisfying

$$
\|\bar{x}(\gamma)\|<\delta_{0} \quad \text { and } \sup _{s \in[\gamma, v]}\left\|\bar{x}(s)-\bar{x}(\gamma)-\int_{\gamma}^{s} D F(\bar{x}(\tau), t)\right\|<\rho,
$$

then

$$
\|\bar{x}(t)\|<\varepsilon, \quad \text { for } t \in[\gamma, v] \cap[\gamma+T,+\infty) \text { and } \gamma \geq t_{0} .
$$

Definition 8.10. The trivial solution $x \equiv 0$ of (8.3.1) is called regularly asymptotically stable, if it is regularly stable and regularly attracting.

Besides the generalized ODE (8.3.1), we consider the perturbed generalized ODE

$$
\frac{d x}{d \tau}=D[F(x, t)+P(t)]
$$

where $F: O \times\left[t_{0}, \infty\right) \rightarrow G\left(\left[t_{0}-r, \infty\right), \mathbb{R}^{n}\right)$ and $P:\left[t_{0}, \infty\right) \rightarrow G\left(\left[t_{0}-r, \infty\right), \mathbb{R}^{n}\right)$ which are given by (8.2.1) and (8.2.2).

Now, consider the following definitions involving the perturbed generalized ODEs.

Definition 8.11. The trivial solution $x \equiv 0$ of (8.3.1) is called regularly stable with respect to perturbations, if for every $\varepsilon>0$, there exists $\delta=\delta(\varepsilon)>0$ such that, if $\left\|x_{0}\right\|<\delta$ and $P \in G^{-}([\gamma, v], X)$ with

$$
\sup _{s \in[\gamma, v]}\|P(s)-P(\gamma)\|<\delta
$$

then

$$
\left\|\bar{x}\left(t ; \gamma, x_{0}\right)\right\|<\varepsilon, \text { for every } t \in[\gamma, v]
$$

where $\bar{x}\left(t ; \gamma, x_{0}\right)$ is a solution of the perturbed generalized ODE (8.3.2), with $\bar{x}\left(\gamma ; \gamma, x_{0}\right)=$ $x_{0}$ and $[\gamma, v] \subset\left[t_{0},+\infty\right)$.

Definition 8.12. The trivial solution $x \equiv 0$ of (8.3.1) is called regularly attracting with respect to perturbations, if there exists $\widetilde{\delta}>0$ and for every $\varepsilon>0$, there exist $T=T(\varepsilon) \geq 0$ 
and $\rho=\rho(\varepsilon)>0$ such that, if

$$
\left\|x_{0}\right\|<\widetilde{\delta} \quad \text { and } \quad \sup _{s \in[\gamma, v]}\|P(s)-P(\gamma)\|<\rho
$$

with $P \in G^{-}([\gamma, v], X)$, then

$$
\left\|\bar{x}\left(t ; \gamma, x_{0}\right)\right\|<\varepsilon, \text { for all } t \geq \gamma+T, t \in[\gamma, v]
$$

where $\bar{x}\left(t ; \gamma, x_{0}\right)$ is a solution of the perturbed generalized ODE (8.3.2) with $\bar{x}\left(\gamma ; \gamma, x_{0}\right)=$ $x_{0}$ and $[\gamma, v] \subset\left[t_{0},+\infty\right)$.

Definition 8.13. The trivial solution $x \equiv 0$ of (8.3.1) is called regularly asymptotically stable with respect to perturbations, if it is both stable and attracting with respect to perturbations.

The following result shows us how the previous concepts of stability can be related. It is an original result which is contained in [38].

Proposition 8.14. The following statements hold.

(i) The trivial solution $x \equiv 0$ of (8.3.1) is regularly stable, if and only if, it is regularly stable with respect to perturbations.

(ii) The trivial solution $x \equiv 0$ of (8.3.1) is regularly attracting, if and only if, it is regularly attracting with respect to perturbations.

(iii) The trivial solution $x \equiv 0$ of (8.3.1) is regularly asymptotically stable, if and only if, it is regularly asymptotically stable with respect to perturbations.

Proof. Let us prove (i). Assume that the trivial solution $x \equiv 0$ of (8.3.1) is regularly stable. Let $\varepsilon>0$ and the quantity $\delta=\delta(\varepsilon)>0$ be given according to Definition 8.11. Also, let $x(t)=x\left(t ; \gamma, x_{0}\right)$ be a solution of the perturbed generalized ODE (8.3.2) on $[\gamma, v]$. Then, by the definition, we obtain, for $s \in[\gamma, v]$,

$$
x(s)-x(\gamma)=\int_{\gamma}^{s} D F(x(\tau), t)+P(s)-P(\gamma) .
$$

Also, suppose $\|x(\gamma)\|=\left\|x\left(\gamma ; \gamma, x_{0}\right)\right\|<\delta$ and $\sup _{s \in[\gamma, v]}\|P(s)-P(\gamma)\|<\delta$. It follows from (8.3.3) that

$$
\sup _{s \in[\gamma, v]}\left\|x(s)-x(\gamma)-\int_{\gamma}^{s} D F(x(\tau), t)\right\| \leq \sup _{s \in[\gamma, v]}\|P(s)-P(\gamma)\|<\delta .
$$


Then, the regularly stability implies

$$
\|x(t)\|=\left\|x\left(t, \gamma, x_{0}\right)\right\|<\varepsilon, \quad \text { for } t \in[\gamma, v]
$$

and the trivial solution of (8.3.1) is regularly stable with respect to perturbations.

Reciprocally, if the trivial solution $x \equiv 0$ of (8.3.1) is regularly stable with respect to perturbations, let $\bar{x}:[\gamma, v] \rightarrow B_{c}, t_{0} \leq \gamma<v<+\infty$, be a regulated function on $[\gamma, v]$ such that

$$
\|\bar{x}(\gamma)\|<\delta \quad \text { and } \quad \sup _{s \in[\gamma, v]}\left\|\bar{x}(s)-\bar{x}(\gamma)-\int_{\gamma}^{s} D F(\bar{x}(\tau), t)\right\|<\delta,
$$

where $\delta>0$ corresponds to some $\varepsilon>0$ from Definition 8.11.

For $s \in[\gamma, v]$, define $P(s)=P(\gamma)+\bar{x}(s)-\bar{x}(\gamma)-\int_{\gamma}^{s} D F(\bar{x}(\tau), t)$. Then, for all $s_{1}, s_{2} \in[\gamma, v]$, we have

$$
\bar{x}\left(s_{2}\right)-\bar{x}\left(s_{1}\right)=\int_{s_{1}}^{s_{2}} D F(\bar{x}(\tau), t)+P\left(s_{2}\right)-P\left(s_{1}\right)
$$

which implies that $\bar{x}$ is a solution of (8.3.2) on $[\gamma, v]$. Moreover,

$$
\sup _{s \in[\gamma, v]}\|P(s)-P(\gamma)\|=\sup _{s \in[\gamma, v]}\left\|\bar{x}(s)-\bar{x}(\gamma)-\int_{\gamma}^{s} D F(\bar{x}(\tau), t)\right\|<\delta .
$$

Thus, the regular stability with respect to perturbations implies $\|\bar{x}(t)\|=\left\|\bar{x}\left(t ; \gamma, x_{0}\right)\right\|<$ $\varepsilon$, for all $t \in[\gamma, v]$. Then it follows that the trivial solution $x \equiv 0$ of (8.3.1) is regularly stable.

Now, assume that the trivial solution $x \equiv 0$ of (8.3.1) is regularly attracting. Then there exists $\widetilde{\delta}>0$ and for every $\varepsilon>0$, there exist $T=T(\varepsilon) \geq 0$ and $\rho=\rho(\varepsilon)>0$ such that if $\bar{x}:[\gamma, v] \rightarrow B_{c}, t_{0} \leq \gamma<v<+\infty$, is a regulated function such that $\|\bar{x}(\gamma)\|<\widetilde{\delta}$ and

$$
\sup _{s \in[\gamma, v]}\left\|\bar{x}(s)-\bar{x}(\gamma)-\int_{\gamma}^{s} D F(\bar{x}(\tau), t)\right\|<\rho,
$$

then

$$
\|\bar{x}(t)\|<\varepsilon, \quad t \in[\gamma, v] \cap[\gamma+T,+\infty), \gamma \geq t_{0} .
$$

Denote the solution of the perturbed generalized ODE (8.3.2) satisfying $x\left(\gamma ; \gamma, x_{0}\right)=$ $x_{0}$ by $x(t)=x\left(t ; \gamma, x_{0}\right)$. Suppose there exists $\widetilde{\delta}>0$ and for every $\varepsilon>0$, there exists 
$\rho=\rho(\varepsilon)>0$ such that $\left\|x_{0}\right\|<\widetilde{\delta}$ and

$$
\sup _{s \in[\gamma, v]}\|P(s)-P(\gamma)\|<\rho
$$

Moreover, suppose $P \in G^{-}([\gamma, v], X)$. Then $\left\|x_{0}\right\|=\|x(\gamma)\|<\widetilde{\delta}$ and, by the definition of a solution of equation (8.3.2), we obtain

$$
\sup _{s \in[\gamma, v]}\left\|x(s)-x(\gamma)-\int_{\gamma}^{s} D F(x(\tau), t)\right\|=\sup _{s \in[\gamma, v]}\|P(s)-P(\gamma)\|<\rho .
$$

Hence, since $x$ is regularly attracting, we have

$$
\left\|x\left(t, \gamma, x_{0}\right)\right\|=\|x(t)\|<\varepsilon, \text { for all } t \geq \gamma+T, t \in[\gamma, v]
$$

that is, the trivial solution $x \equiv 0$ of $(8.3 .1)$ is regularly attracting with respect to perturbations.

Reciprocally, we assume that the trivial solution $x \equiv 0$ of (8.3.1) is regularly attracting with respect to perturbations. Let $\bar{x}:[\gamma, v] \rightarrow B_{c}, t_{0} \leq \gamma<v<+\infty$, be a regulated on $[\gamma, v]$ satisfying

$$
\|\bar{x}(\gamma)\|<\widetilde{\delta} \text { and } \sup _{s \in[\gamma, v]}\left\|\bar{x}(s)-\bar{x}(\gamma)-\int_{\gamma}^{s} D F(\bar{x}(\tau), t)\right\|<\rho .
$$

Again, for $s \in[\gamma, v]$, define $P(s)=P(\gamma)+\bar{x}(s)-\bar{x}(\gamma)-\int_{\gamma}^{s} D F(\bar{x}(\tau), t)$. Then $\bar{x}(t)$ is a solution of (8.3.2) on $[\gamma, v]$. Thus,

$$
\sup _{s \in[\gamma, v]}\|P(s)-P(\gamma)\|<\rho
$$

Therefore, Definition 8.12 yields

$$
\|\bar{x}(t)\|<\varepsilon, \quad t \in[\gamma, v] \cap[\gamma+T,+\infty), \gamma \geq t_{0} .
$$

which implies the regular attractivity of the trivial solution of (8.3.1).

Item (iii) follows from (i) and (ii) and the proof is complete. 


\subsection{Relations between stability concepts for measure FDEs and generalized ODEs}

In this section, we present some results which establish relations between stability concepts for generalized ODEs and the corresponding stability concepts for measure FDEs. In order to obtain these results, we use the correspondence between these types of equations described by Theorem 8.7 .

Let $O \subset G\left(\left[t_{0}-r,+\infty\right), \mathbb{R}^{n}\right)$ with the prolongation property and $P=\left\{y_{t}, y \in O, t \in\right.$ $\left.\left[t_{0}, \infty\right)\right\} \subset G\left([-r, 0], \mathbb{R}^{n}\right)$. Consider the measure FDE

$$
D x=f\left(x_{t}, t\right) D g,
$$

where $f: P \times\left[t_{0},+\infty\right) \rightarrow \mathbb{R}^{n}$ satisfies conditions $\left(H_{1}\right),\left(H_{2}\right)$ and $\left(H_{3}\right)$ and $g:\left[t_{0},+\infty\right] \rightarrow \mathbb{R}$ is a nondecreasing and left-continuous function. Also, assume that $D x$ and $D g$ are the distributional derivatives with respect to $x$ and $g$ in the sense of L. Schwartz and $f(0, t)=0$ for every $t \in\left[t_{0},+\infty\right)$, which implies that $y \equiv 0$ is a solution of (8.4.1).

Consider the function

$$
F: O \times\left[t_{0},+\infty\right) \rightarrow G\left(\left[t_{0}-r,+\infty\right), \mathbb{R}^{n}\right)
$$

defined by

$$
F(y, t)(\vartheta)=\left\{\begin{array}{l}
0, \quad t_{0}-r \leq \vartheta \leq t_{0}, \\
\int_{t_{0}}^{\vartheta} f\left(y_{s}, s\right) \mathrm{d} g(s), \quad t_{0} \leq \vartheta \leq t<+\infty, \\
\int_{t_{0}}^{t} f\left(y_{s}, s\right) \mathrm{d} g(s), \quad t \leq \vartheta<+\infty .
\end{array}\right.
$$

Since $f(0, t)=0$, for every $t \in\left[t_{0}, \infty\right), F(0, t)=0$, for all $t \in\left[t_{0},+\infty\right)$, which implies that $x \equiv 0$ is a solution of the generalized differential equation

$$
\frac{d x}{d \tau}=D F(x, t)
$$

on $\left[t_{0},+\infty\right)$.

Further, we consider the perturbed measure FDE

$$
D y=f\left(y_{t}, t\right) D g+p(t) D u,
$$


where $p:\left[t_{0}, \infty\right) \rightarrow \mathbb{R}^{n}$ satisfies conditions $\left(H_{4}\right)$ and $\left(H_{5}\right)$ and $u:\left[t_{0}, \infty\right) \rightarrow \mathbb{R}$ is a nondecreasing and left-continuous function. Moreover, $D u$ is the distributional derivatives in the sense of L. Schwartz with respect to $u$. By Theorem 8.7, it is possible to associate an initial value problem for equation (8.4.4) with initial value problem for the following perturbed generalized ODE

$$
\frac{d x}{d \tau}=D G(x, t)=D[F(x, t)+P(t)]
$$

where $F: O \times\left[t_{0},+\infty\right) \rightarrow G^{-}\left(\left[t_{0}-r,+\infty\right), \mathbb{R}^{n}\right)$ and $P:\left[t_{0},+\infty\right) \rightarrow G^{-}\left(\left[t_{0}-r,+\infty\right), \mathbb{R}^{n}\right)$ are given by (8.4.2) and

$$
P(t)(\vartheta)=\left\{\begin{array}{l}
0, \quad t_{0}-r \leq \vartheta \leq t_{0} \\
\int_{t_{0}}^{\vartheta} p(s) \mathrm{d} u(s), \quad t_{0} \leq \vartheta \leq t<+\infty \\
\int_{t_{0}}^{t} p(s) \mathrm{d} u(s), \quad t \leq \vartheta<+\infty .
\end{array}\right.
$$

We are now able to present a result which relates the concepts of regularly stability and regularly attractivity of the trivial solution of measure FDEs and the corresponding concepts for generalized ODEs. Such result is new. See [38].

Theorem 8.15. Suppose the function $f: P \times\left[t_{0}, \infty\right) \rightarrow \mathbb{R}^{n}$ satisfies conditions $\left(H_{1}\right)$, $\left(H_{2}\right)$ and $\left(H_{3}\right)$. Assume that the functions $g, u:\left[t_{0}, \infty\right) \rightarrow \mathbb{R}$ are nondecreasing and leftcontinuous. Also, the function $p:\left[t_{0}, \infty\right) \rightarrow \mathbb{R}^{n}$ satisfies conditions $\left(H_{4}\right)$ and $\left(H_{5}\right)$. Then the following statements hold:

(i) The trivial solution $y \equiv 0$ of (8.4.1) is integrally stable, if and only if, the trivial solution $x \equiv 0$ of $(8.4 .3)$ is regularly stable.

(ii) If the trivial solution $x \equiv 0$ of (8.4.3) is regularly attracting, then the trivial solution $y \equiv 0$ of (8.4.1) is integrally attracting.

(iii) If the trivial solution $x \equiv 0$ of (8.4.3) is regularly asymptotically stable, then the trivial solution $y \equiv 0$ of (8.4.1) is integrally asymptotically stable.

Proof. We start by proving (i). Suppose the trivial solution of (8.4.1) is integrally stable. Then, given $\varepsilon>0$, there exists $\delta=\delta(\varepsilon)>0$ such that if $\phi \in P$ with $\|\phi\|_{\infty}<\delta$ and

$$
\sup _{t \in[\gamma, v]}\left|\int_{\gamma}^{t} p(s) \mathrm{d} u(s)\right|<\delta,
$$


then

$$
\left|\bar{y}_{t}(\gamma, \phi)\right|<\frac{\varepsilon}{2}, \quad t \in[\gamma, v]
$$

where $\bar{y}(t ; \gamma, \phi)$ is a solution of (8.4.4) such that $\bar{y}_{\gamma}=\phi$.

We will prove that the trivial solution of generalized equation (8.4.3), with $F$ given by (8.2.1), is regularly stable with respect to perturbations, then the result will follow by Proposition 8.14 .

Let $x\left(t ; \gamma, x_{0}\right)$ be a solution of the perturbed generalized ODE (8.4.5) with the initial condition $x\left(\gamma ; \gamma, x_{0}\right)=x_{0}$, where $F$ and $P$ are given by (8.2.1) and (8.2.2), respectively. Let $\varepsilon>0$ and suppose there exists $\delta=\delta(\varepsilon)>0$ such that $\delta<\varepsilon$ and, moreover, assume that

$$
\left\|x_{0}\right\|_{\infty}<\delta \text { and } \sup _{t \in[\gamma, v]}\|P(t)-P(\gamma)\|<\delta,
$$

where $x_{0} \in G^{-}\left(\left[t_{0}-r, \infty\right), \mathbb{R}^{n}\right)$ and $P \in G^{-}\left([\gamma, v], \mathbb{R}^{n}\right)$.

We have $\|x(\gamma)\|=\left\|x\left(\gamma ; \gamma, x_{0}\right)\right\|=\left\|x_{0}\right\|<\delta$ which means that $\sup _{\theta \in[\gamma-r,+\infty)}|x(\gamma)(\theta)|<$ $\delta$ and, therefore, $\sup _{\theta \in[\gamma-r, \gamma]}|\phi(\theta-\gamma)|<\delta / 2$, by the definition of initial condition of equation (8.4.5). Thus,

$$
\|\phi\|_{\infty}<\delta / 2
$$

Since $x$ is a solution of the perturbed generalized ODE on $[\gamma, v]$, we have

$$
x\left(u_{2}\right)-x\left(u_{1}\right)=\int_{u_{1}}^{u_{2}} D F(x(\tau), s)+P\left(u_{2}\right)-P\left(u_{1}\right),
$$

for every $u_{1}, u_{2} \in[\gamma, v]$.

Therefore,

$$
\sup _{t \in[\gamma, v]}\left\|x(t)-x(\gamma)-\int_{\gamma}^{t} D F(x(\tau), s)\right\|=\sup _{t \in[\gamma, v]}\|P(t)-P(\gamma)\|<\delta / 2 .
$$


Then, it follows that

$$
\begin{aligned}
\|P(t)-P(\gamma)\| & =\sup _{\vartheta \in[\gamma-r, t]}|P(t)(\vartheta)-P(\gamma)(\vartheta)| \\
& \geq \sup _{\vartheta \in[\gamma, t]}|P(t)(\vartheta)-P(\gamma)(\vartheta)| \\
& =\sup _{\vartheta \in[\gamma, t]}\left|\int_{\gamma}^{\vartheta} p(s) \mathrm{d} u(s)\right| \\
& \geq\left|\int_{\gamma}^{t} p(s) \mathrm{d} u(s)\right| .
\end{aligned}
$$

Then, we get

$$
\sup _{t \in[\gamma, v]}\left|\int_{\gamma}^{t} p(s) \mathrm{d} u(s)\right| \leq \frac{\delta}{2} .
$$

Thus, by the integral stability of the trivial solution of (8.4.1), $|y(t)|<\varepsilon / 2$, for all $t \in[\gamma, v]$.

Finally, for $t \in[\gamma, v]$, we have

$$
\|x(t)\|_{\infty}=\sup _{\theta \in[\gamma-r,+\infty)}|x(t)(\theta)|=\sup _{\theta \in[\gamma-r, t]}|y(\theta)|=\left\|y_{s}\right\|_{\infty}<\varepsilon
$$

for $s \in[\gamma, t]$, since $\delta<\varepsilon$ and we have the sufficiency of item (i).

Now, using (i) from Proposition 8.14, we assume that the trivial solution of (8.4.3) is regularly stable with respect to perturbations. Thus, given $\varepsilon>0$, let $\delta=\delta(\varepsilon)>0$ be the quantity from Definition 8.11.

Let $y(t ; \gamma, \phi)$ be a solution of the perturbed FDE (8.4.4). Suppose $\phi \in P$ with $\|\phi\|_{\infty}<$ $\delta$ and $\sup _{t \in[\gamma, v]}\left|\int_{\gamma}^{t} p(s) \mathrm{d} u(s)\right|<\delta / 2$. We want to prove that $y \equiv 0$ is integrally stable, that is, $|y(t ; \gamma, \phi)|<\varepsilon, t \in[\gamma, v]$.

Let $x\left(t ; \gamma, x_{0}\right)$ be the solution of the perturbed generalized ODE (8.4.5) with $F$ given by (8.4.2) and $P$ given by (8.4.6), that is, $x$ is the solution corresponding to $y$ obtained according to Theorem 8.7 .

By the definition of the function $P$ and from the fact that $\sup _{t \in[\gamma, v]}\left|\int_{\gamma}^{t} p(s) \mathrm{d} u(s)\right|<$ $\delta / 2$, we obtain

$$
\sup _{t \in[\gamma, v]}\|P(t)-P(\gamma)\|<\delta / 2 .
$$

Thus, from the regular stability with respect to perturbations of the trivial solution of 
(8.4.5), we obtain $\|x(t)\|<\varepsilon$, which implies

$$
\sup _{\theta \in[\gamma-r, v]}|x(t)(\theta)|<\varepsilon, \quad t \in[\gamma, v] .
$$

Therefore, the relations in Theorem 8.7 imply

$$
\sup _{\theta \in[\gamma-r, t]}|y(\theta)|<\varepsilon, \quad t \in[\gamma, v] .
$$

In particular,

$$
\sup _{\theta \in[\gamma, v]}|y(\theta)| \leq \sup _{\theta \in[\gamma-r, v]}|y(\theta)|<\varepsilon,
$$

and the desired result follows.

Now, we will prove (ii). Suppose the trivial solution of generalized ODE (8.4.3) is regularly attracting with respect to perturbations. Then, there exists $\widetilde{\delta}>0$ and given $\varepsilon>0$, there exist $T=T(\varepsilon) \geq 0$ and $\rho=\rho(\varepsilon)>0$ satisfying the conditions from Definition 8.12 .

Let $y(t ; \gamma, \phi)$ be a solution of the perturbed measure FDE (8.4.4). Suppose $\|\phi\|_{\infty}<\widetilde{\delta}$ and $\sup _{t \in[\gamma, v]}\left|\int_{\gamma}^{t} p(s) \mathrm{d} u(s)\right|<\rho$. Then, it follows that

$$
\sup _{t \in[\gamma, v]}\|P(t)-P(\gamma)\|<\rho
$$

By Theorem 8.7, it follows that $\left\|x_{0}\right\|_{\infty}=\|\phi\|_{\infty}<\widetilde{\delta}$. Therefore, the regularly attractivity with respect to perturbations of the trivial solution of (8.4.3) implies

$$
\|x(t)\|_{\infty}=\left\|x\left(t ; \gamma, x_{0}\right)\right\|_{\infty}<\varepsilon, \quad t \geq \gamma+T, t \in[\gamma, v] .
$$

Therefore, for $t \geq \gamma+T, T>r$ and $t \in[\gamma, v]$, we have by Theorem 8.7,

$$
|y(t)|=|y(t ; \gamma, \phi)|=|x(t)(t)| \leq\|x(t)\|_{\infty}<\varepsilon,
$$

and we have the desired result.

Note that assertion (iii) follows directly from (i), (ii) and from Proposition 8.14. 


\subsection{Stability for impulsive measure FDEs}

In this section, we introduce some new definitions concerning stability of the trivial solution of impulsive measure functional differential equations and we present a result which relates these concepts with the corresponding ones for measure FDEs, using the correspondence between the equations presented in Chapter 4. Also, as a consequence, we present a result which relates these stability concepts to the corresponding ones for generalized ODEs.

Throughout this section, consider $O \subset G\left(\left[t_{0}-r,+\infty\right), \mathbb{R}^{n}\right)$ with the prolongation property and $P=\left\{y_{t} ; y \in O, t \in\left[t_{0}, \infty\right)\right\} \subset G\left([-r, 0], \mathbb{R}^{n}\right)$. Consider that the function $g:\left[t_{0},+\infty\right) \rightarrow \mathbb{R}$ is nondecreasing.

We assume the following conditions concerning the function $f: P \times\left[t_{0},+\infty\right) \rightarrow \mathbb{R}^{n}$ :

$\left(H_{1}\right)$ The integral $\int_{t_{0}}^{t} f\left(y_{s}, s\right) \mathrm{d} g(s)$ exists for every $y \in O$ and every $t \in\left[t_{0}, \infty\right)$.

$\left(H_{2}\right)$ There exists a constant $M>0$ such that the inequality

$$
\left|\int_{u}^{v} f\left(y_{s}, s\right) \mathrm{d} g(s)\right| \leq M[g(v)-g(u)]
$$

holds for every $y \in O$ and every $u, v \in\left[t_{0}, \infty\right)$.

$\left(H_{3}\right)$ There exists a constant $L>0$ such that the inequality

$$
\left|\int_{u}^{v}\left[f\left(y_{s}, s\right)-f\left(z_{s}, s\right)\right] \mathrm{d} g(s)\right| \leq L \int_{u}^{v}\left\|y_{s}-z_{s}\right\|_{\infty} \mathrm{d} g(s)
$$

holds for every $y, z \in O$ and every $u, v \in\left[t_{0}, \infty\right)$.

Let $B \subset \mathbb{R}^{n}$ be open. We consider the following conditions on the impulse operators $I_{k}: \mathbb{R}^{n} \rightarrow \mathbb{R}^{n}, k \in\{1,2, \ldots, m\}:$

$\left(H_{6}\right)$ There exists a constant $K_{1}>0$ such that

$$
\left|I_{k}(x)\right| \leq K_{1}
$$

for every $k \in\{1, \ldots, m\}$ and $x \in B$. 
$\left(H_{7}\right)$ There exists a constant $K_{2}>0$ such that

$$
\left|I_{k}(x)-I_{k}(y)\right| \leq K_{2}|x-y|
$$

for every $k \in\{1, \ldots, m\}$ and $x, y \in B$.

In what follows, we introduce some new concepts of stability of the trivial solution for impulsive measure FDEs with perturbations. Let us start by considering an impulsive equation

$$
x(t)=x\left(t_{0}\right)+\int_{t_{0}}^{t} f\left(x_{s}, s\right) \mathrm{d} g(s)+\sum_{t_{0} \leq t_{k}<t} I_{k}\left(x\left(t_{k}\right)\right), \quad t \in\left[t_{0}, \infty\right)
$$

and its perturbed impulsive equation

$$
x(t)=x\left(t_{0}\right)+\int_{t_{0}}^{t} f\left(x_{s}, s\right) \mathrm{d} g(s)+\int_{t_{0}}^{t} p(s) \mathrm{d} u(s)+\sum_{t_{0} \leq t_{k}<t} I_{k}\left(x\left(t_{k}\right)\right), \quad t \in\left[t_{0}, \infty\right) .
$$

Definition 8.16. The trivial solution $y \equiv 0$ of (8.5.1) is said to be integrally stable, if for every $\varepsilon>0$ there is a $\delta=\delta(\varepsilon)>0$ such that if $\phi \in P$ with $\|\phi\|_{\infty}<\delta$ and

$$
\sup _{t \in[\gamma, v]}\left|\int_{\gamma}^{t} p(s) \mathrm{d} u(s)\right|<\delta,
$$

then

$$
\left|\bar{y}_{t}(\gamma, \phi)\right|<\varepsilon, \text { for every } t \in[\gamma, v] \text {, }
$$

where $\bar{y}(t ; \gamma, \phi)$ is a solution of the perturbed impulsive equation (8.5.2) satisfying $\bar{y}_{\gamma}=\phi$.

Definition 8.17. The trivial solution $y \equiv 0$ of (8.5.1) is called integrally attracting, if there is $\widetilde{\delta}>0$ and for every $\varepsilon>0$, there exist $T=T(\varepsilon) \geq 0$ and $\rho=\rho(\varepsilon)>0$ such that if

$$
\|\phi\|_{\infty}<\widetilde{\delta} \quad \text { and } \quad \sup _{t \in[\gamma, v]}\left|\int_{\gamma}^{t} p(s) \mathrm{d} u(s)\right|<\rho
$$

then

$$
\left|\bar{y}_{t}(\gamma, \phi)\right|<\varepsilon, \text { for all } t \geq \gamma+T, t \in[\gamma, v]
$$

where $\bar{y}(t ; \gamma, \phi)$ is a solution of the perturbed impulsive equation (8.5.2) satisfying $\bar{y}_{\gamma}=\phi$. Definition 8.18. The trivial solution $y \equiv 0$ of (8.5.1) is called integrally asymptotically stable, if it is integrally stable and integrally attracting.

The next result describes the relations between these new concepts of stability for 
impulsive measure FDEs and the corresponding ones for measure FDEs. Such result is new.

Theorem 8.19. Let $f: P \times[\gamma,+\infty) \rightarrow \mathbb{R}^{n}$ be a function satisfying $\left(H_{1}\right)$ and $p:$ $\left[t_{0},+\infty\right) \rightarrow \mathbb{R}^{n}$ be a function satisfying $\left(H_{4}\right)$. Also, consider that the functions $u, g$ : $\left[t_{0}, \infty\right) \rightarrow \mathbb{R}$ are nondecreasing and left-continuous at every point and continuous at $\left\{t_{k}\right\}_{k \in \mathbb{N}}$, where $t_{k}, k \in \mathbb{N} \backslash\{0\}$, are the moments of impulses. Moreover, let $I_{k}: \mathbb{R}^{n} \rightarrow \mathbb{R}^{n}$, $k \in \mathbb{N}$, be the impulses operators. Then, the following statements hold.

(i) The trivial solution $y \equiv 0$ of (8.4.1) is integrally stable, if and only if, the trivial solution $y \equiv 0$ of (8.5.1) is integrally stable.

(ii) The trivial solution $y \equiv 0$ of (8.4.1) is integrally attracting, if and only if, the trivial solution $y \equiv 0$ of (8.5.1) is integrally attracting.

(iii) The trivial solution $y \equiv 0$ of (8.4.1) is integrally asymptotically stable, if and only if, the trivial solution $y \equiv 0$ of (8.5.1) is integrally asymptotically stable.

Proof. At first, define the functions $\tilde{u}, \tilde{g}:\left[t_{0}, \infty\right) \rightarrow \mathbb{R}$ by

$$
\tilde{u}(t)=\left\{\begin{array}{l}
u(t), \quad t \in\left[t_{0}, t_{1}\right] \\
u(t)+c_{k}, \quad t \in\left(t_{k}, t_{k+1}\right] \text { for some } k \in \mathbb{N}
\end{array}\right.
$$

and

$$
\tilde{g}(t)=\left\{\begin{array}{l}
g(t), \quad t \in\left[t_{0}, t_{1}\right] \\
g(t)+d_{k}, \quad t \in\left(t_{k}, t_{k+1}\right] \text { for some } k \in \mathbb{N}
\end{array}\right.
$$

where the sequences $\left\{c_{k}\right\}_{k=1}^{\infty}$ and $\left\{d_{k}\right\}_{k=1}^{\infty}$ are chosen in such way that $\Delta^{+} \tilde{u}\left(t_{k}\right)=1$ and $\Delta^{+} \tilde{g}\left(t_{k}\right)=1$ for every $k \in \mathbb{N}$.

It follows directly from the definitions (8.5.3) and (8.5.4) that the functions $\tilde{u}$ and $\tilde{g}$ are nondecreasing and left continuous.

Also, we define the following functions

$$
\tilde{f}(y, t)=\left\{\begin{array}{l}
f(y, t), \quad t \in\left[t_{0}, \infty\right) \backslash\left\{t_{k}\right\}_{k=1}^{\infty} \\
I_{k}(y(0)), \quad t=t_{k} \text { for some } k \in \mathbb{N}
\end{array}\right.
$$

and

$$
\tilde{p}(t)=\left\{\begin{array}{l}
p(t), \quad t \in\left[t_{0}, \infty\right) \backslash\left\{t_{k}\right\}_{k=1}^{\infty} \\
0, \quad t=t_{k} \text { for some } k \in \mathbb{N}
\end{array}\right.
$$


By the equalities (8.5.3), (8.5.4), (8.5.5) and (8.5.6) and by the hypotheses, we obtain, using Theorem 4.16 , that $\bar{y}$ is a solution of

$$
\bar{y}(t)=\bar{y}\left(t_{0}\right)+\int_{t_{0}}^{t} f\left(\bar{y}_{s}, s\right) \mathrm{d} g(s)+\int_{t_{0}}^{t} p(s) \mathrm{d} u(s)+\sum_{t_{0} \leq t_{k}<t} I_{k}\left(\bar{y}\left(t_{k}\right)\right), \quad t \in\left[t_{0}, \infty\right),
$$

if and only if, $\bar{y}$ is also a solution of

$$
\bar{y}(t)=\bar{y}\left(t_{0}\right)+\int_{t_{0}}^{t} \tilde{f}\left(\bar{y}_{s}, s\right) \mathrm{d} \tilde{g}(s)+\int_{t_{0}}^{t} \tilde{p}(s) \mathrm{d} \tilde{u}(s), \quad t \in\left[t_{0}, \infty\right) .
$$

Moreover, by the equalities (8.5.4) and (8.5.5) and by hypotheses, we obtain, using Theorem 4.16, that $y$ is a solution of

$$
y(t)=y\left(t_{0}\right)+\int_{t_{0}}^{t} f\left(y_{s}, s\right) \mathrm{d} g(s)+\sum_{t_{0} \leq t_{k}<t} I_{k}\left(y\left(t_{k}\right)\right), \quad t \in\left[t_{0}, \infty\right),
$$

if and only if, $y$ is a solution of

$$
y(t)=y\left(t_{0}\right)+\int_{t_{0}}^{t} \tilde{f}\left(y_{s}, s\right) \mathrm{d} \tilde{g}(s), \quad t \in\left[t_{0}, \infty\right) .
$$

Let us prove item (i). Suppose the solution of (8.4.1) is integrally stable and, moreover, for every $\varepsilon>0$ there is $\delta=\delta(\varepsilon)>0$ such that $\phi \in P$,

$$
\|\phi\|_{\infty}<\delta \text { and } \sup _{t \in[\gamma, v]}\left|\int_{\gamma}^{t} p(s) \mathrm{d} u(s)\right|<\delta
$$

Then, it is clear from the definitions of the functions $\tilde{p}$ and $\tilde{u}$ that

$$
\sup _{t \in[\gamma, v]}\left|\int_{\gamma}^{t} \tilde{p}(s) \mathrm{d} \tilde{u}(s)\right|<\delta .
$$

Thus, since the solution of (8.4.1) is integrally stable, we obtain

$$
\left|\bar{y}_{t}(\gamma, \phi)\right|<\varepsilon, \text { for every } t \in[\gamma, v] \text {, }
$$

where $\bar{y}(t ; \gamma, \phi)$ is a solution of impulsive equation with perturbation (8.5.8) satisfying $\bar{y}_{\gamma}=\phi$. By Theorem 4.16, $\bar{y}(t ; \gamma, \phi)$ is also a solution of (8.5.7) satisfying $\bar{y}_{\gamma}=\phi$.

Furthermore, since (8.5.11) is fulfilled, it follows from the definition that the trivial 
solution $y \equiv 0$ of (8.5.1) is integrally stable.

Reciprocally, suppose the trivial solution $y \equiv 0$ of (8.5.1) is integrally stable and for every $\varepsilon>0$, there is $\delta=\delta(\varepsilon)>0$ such that $\phi \in P$,

$$
\|\phi\|_{\infty}<\delta \text { and } \sup _{t \in[\gamma, v]}\left|\int_{\gamma}^{t} \tilde{p}(s) \mathrm{d} \tilde{u}(s)\right|<\delta .
$$

Again, by the definition of functions $\tilde{p}$ and $\tilde{u}$ given by (8.5.6) and (8.5.3), respectively, it is clear that

$$
\sup _{t \in[\gamma, v]}\left|\int_{\gamma}^{t} p(s) \mathrm{d} u(s)\right|<\delta .
$$

Thus, it follows immediately from the definition that

$$
\left|\bar{y}_{t}(\gamma, \phi)\right|<\varepsilon, \text { for every } t \in[\gamma, v]
$$

where $\bar{y}(t ; \gamma, \phi)$ is a solution of the perturbed impulsive equation (8.5.7) satisfying $\bar{y}_{\gamma}=\phi$.

By the correspondence between the solutions of initial value problems for (8.5.7) and (8.5.8) (see Theorem 4.16), $\bar{y}(t ; \gamma, \phi)$ is also a solution of (8.5.8) satisfying $\bar{y}_{\gamma}=\phi$. Thus, by the definition, the trivial solution of (8.4.1) is integrally stable and item (i) follows.

Now, consider the item (ii). Suppose the trivial solution $y \equiv 0$ of (8.4.1) is integrally attracting and assume that there is $\widetilde{\delta}>0$ and for every $\varepsilon>0$, there exist $T=T(\varepsilon) \geq 0$ and $\rho=\rho(\varepsilon)>0$ such that

$$
\|\phi\|_{\infty}<\widetilde{\delta} \quad \text { and } \quad \sup _{t \in[\gamma, v]}\left|\int_{\gamma}^{t} \tilde{p}(s) \mathrm{d} \tilde{u}(s)\right|<\rho .
$$

Using the definition of the functions $\tilde{p}$ and $\tilde{u}$, we obtain

$$
\sup _{t \in[\gamma, v]}\left|\int_{\gamma}^{t} p(s) \mathrm{d} u(s)\right|<\rho,
$$

which implies, by hypothesis, that

$$
\left|\bar{y}_{t}(\gamma, \phi)\right|<\varepsilon, \text { for all } t \geq \gamma+T, t \in[\gamma, v]
$$

where $\bar{y}(t ; \gamma, \phi)$ is a solution of the perturbed equation (8.5.8), satisfying $\bar{y}_{\gamma}=\phi$. Then, using the correspondence between the solution of (8.5.8) and the solution of (8.5.7), described in Theorem $4.16, \bar{y}(t ; \gamma, \phi)$ is a solution of (8.5.7) satisfying $\bar{y}_{\gamma}=\phi$ and therefore, 
by definition, it follows that the solution $y \equiv 0$ of (8.5.1) is integrally attracting.

Reciprocally, suppose the trivial solution $y \equiv 0$ of (8.5.1) is integrally attracting and assume that there is $\widetilde{\delta}>0$ and for every $\varepsilon>0$, there exist $T=T(\varepsilon) \geq 0$ and $\rho=\rho(\varepsilon)>0$ such that

$$
\|\phi\|_{\infty}<\widetilde{\delta} \quad \text { and } \quad \sup _{t \in[\gamma, v]}\left|\int_{\gamma}^{t} p(s) \mathrm{d} u(s)\right|<\rho .
$$

Then, by the definition of the functions $\tilde{p}$ and $\tilde{u}$, we have

$$
\sup _{t \in[\gamma, v]}\left|\int_{\gamma}^{t} \tilde{p}(s) \mathrm{d} \tilde{u}(s)\right|<\rho,
$$

which implies, by hypothesis,

$$
\left|\bar{y}_{t}(\gamma, \phi)\right|<\varepsilon, \text { for all } t \geq \gamma+T, t \in[\gamma, v]
$$

where $\bar{y}(t ; \gamma, \phi)$ is a solution of the perturbed impulsive equation (8.5.7), satisfying $\bar{y}_{\gamma}=\phi$. The result follows immediately using the correspondence between the solution of $(8.5 .8)$ and the solution of (8.5.7) and by (8.5.13).

Item (iii) follows immediately by items (i) and (ii).

Combining the previous theorem, Theorems 4.16, 8.15 and Lemma 4.18, we obtain immediately the next result which shows some relations between the stability concepts for impulsive measure FDEs and the corresponding stability concepts for generalized ODEs. Such result is original. See [38].

Corollary 8.20. Suppose the functions $u, g:\left[t_{0}, \infty\right) \rightarrow \mathbb{R}$ are nondecreasing, leftcontinuous at every point and continuous at $\left\{t_{k}\right\}_{k \in \mathbb{N}}$, where $t_{k}, k \in \mathbb{N}$, are the moments of impulses. Also, suppose the function $f: P \times[\gamma, \infty) \rightarrow \mathbb{R}^{n}$ satisfies conditions $\left(H_{1}\right),\left(H_{2}\right)$ and $\left(H_{3}\right)$ and the function $p:\left[t_{0}, \infty\right) \rightarrow \mathbb{R}^{n}$ satisfies conditions $\left(H_{4}\right)$ and $\left(H_{5}\right)$. Moreover, suppose, for each $k \in \mathbb{N}$, the impulses operators $I_{k}: \mathbb{R}^{n} \rightarrow \mathbb{R}^{n}$ satisfy conditions $\left(H_{6}\right)$ and $\left(H_{7}\right)$ with the same constants for every $k \in \mathbb{N}$. Then the following statements hold:

(i) The trivial solution $z \equiv 0$ of (8.5.1) is integrally stable, if and only if, the trivial solution $x \equiv 0$ of (8.4.3) is regularly stable.

(ii) If the trivial solution $x \equiv 0$ of (8.4.3) is regularly attracting, then the trivial solution $z \equiv 0$ of (8.5.1) is integrally attracting. 
(iii) If the trivial solution $x \equiv 0$ of (8.4.3) is regularly asymptotically stable, then the trivial solution $z \equiv 0$ of (8.5.1) is integrally asymptotically stable.

\subsection{Stability for impulsive functional dynamic equations on time scales}

In this section, we introduce some new definitions concerning stability of the trivial solution of impulsive functional dynamic equations on time scales. Also, we prove one result which relates the new stability concepts for impulsive functional dynamic equations on time scales and the corresponding concepts for impulsive measure FDEs. As an immediate consequence, we can relate stability concepts for impulsive functional dynamic equations on time scales and stability concepts for generalized ODEs.

Let $\mathbb{T}$ be a time scale and $t_{0} \in \mathbb{T}$. Consider the impulsive functional dynamic system on time scales

$$
x(t)=x\left(t_{0}\right)+\int_{t_{0}}^{t} f\left(x_{s}, s\right) \Delta s+\sum_{t_{0} \leq t_{k}<t} I_{k}\left(x\left(t_{k}\right)\right), \quad t \in\left[t_{0}, \infty\right)_{\mathbb{T}}
$$

and the perturbed impulsive functional dynamic system on time scales:

$$
x(t)=x\left(t_{0}\right)+\int_{t_{0}}^{t} f\left(x_{s}, s\right) \Delta s+\int_{t_{0}}^{t} p(s) \Delta s+\sum_{t_{0} \leq t_{k}<t} I_{k}\left(x\left(t_{k}\right)\right), \quad t \in\left[t_{0}, \infty\right)_{\mathbb{T}},
$$

where the moments of impulses $t_{k} \in \mathbb{T}$ are right-dense points.

In the sequel, we introduce new concepts concerning stability of the trivial solution of (8.6.1).

Definition 8.21. The trivial solution $y \equiv 0$ of (8.6.1) is said to be integrally stable, if for every $\varepsilon>0$, there is $\delta=\delta(\varepsilon)>0$ such that if $\phi:[\gamma-r, \gamma]_{\mathbb{T}} \rightarrow \mathbb{R}^{n}$ with $\|\phi\|_{\infty}<\delta$ and

$$
\sup _{t \in[\gamma, v]_{\mathbb{T}}}\left|\int_{\gamma}^{t} p(s) \Delta s\right|<\delta
$$

where $t_{0} \leq \gamma<v<\infty, \quad \gamma, v \in \mathbb{T}$, then

$$
\left|\bar{y}_{t}(\gamma, \phi)\right|<\varepsilon, \text { for every } t \in[\gamma, v]_{\mathbb{T}},
$$


where $\bar{y}(t ; \gamma, \phi)$ is a solution of the perturbed impulsive equation on time scales (8.6.2) satisfying $\bar{y}(t)=\phi(t)$, for $t \in[\gamma-r, \gamma]_{\mathbb{T}}$.

Definition 8.22. The trivial solution $y \equiv 0$ of (8.6.1) is called integrally attracting, if there is $\widetilde{\delta}>0$ and for every $\varepsilon>0$, there exist $T=T(\varepsilon) \geq 0$ and $\rho=\rho(\varepsilon)>0$ such that if

$$
\|\phi\|_{\infty}<\widetilde{\delta} \quad \text { and } \quad \sup _{t \in[\gamma, v]_{\mathbb{T}}}\left|\int_{\gamma}^{t} p(s) \Delta s\right|<\rho,
$$

where $t_{0} \leq \gamma<v<\infty, \gamma, v \in \mathbb{T}$, then

$$
\left|\bar{y}_{t}(\gamma, \phi)\right|<\varepsilon, \quad \text { for all } t \geq \gamma+T, t \in[\gamma, v]_{\mathbb{T}}
$$

where $\bar{y}(t ; \gamma, \phi)$ is a solution of the perturbed impulsive equation on time scales (8.6.2) satisfying $\bar{y}(t)=\phi(t)$, for $t \in[\gamma-r, \gamma]_{\mathbb{T}}$.

Definition 8.23. The trivial solution $y \equiv 0$ of (8.6.1) is called integrally asymptotically stable, if it is integrally stable and integrally attracting.

Theorem 8.24. Let $t_{k} \in \mathbb{T}$, for each $k=0,1,2, \ldots$ and also, let the moments of impulses be right-dense points. Also, assume that for every $y \in G=G\left(\left[t_{0}-r, t_{0}+\eta\right]_{\mathbb{T}}, B\right)$, where $B \subset \mathbb{R}^{n}$ open, the function $t \mapsto f\left(y_{t}^{*}, t\right)$ is Kurzweil-Henstock delta-integrable on $\left[t_{0}, t_{0}+\right.$ $\eta]_{\mathbb{T}}$. Moreover, suppose the function $t \mapsto p(t)$ is Kurzweil-Henstock delta-integrable on $\left[t_{0}, t_{0}+\eta\right]_{\mathbb{T}}$. Define the functions $g(s)=s^{*}$ and $h(s)=s^{*}$ for every $s \in\left[t_{0}, t_{0}+\eta\right]$. Then the following statements hold:

(i) The trivial solution $y^{*} \equiv 0$ of (8.5.1) is integrally stable, if and only if, the trivial solution $y \equiv 0$ of (8.6.1) is integrally stable.

(ii) The trivial solution $y^{*} \equiv 0$ of (8.5.1) is integrally attracting, if and only if, the trivial solution $y \equiv 0$ of (8.6.1) is integrally attracting.

(iii) The trivial solution $y^{*} \equiv 0$ of (8.5.1) is integrally asymptotically stable, if and only if, the trivial solution $y \equiv 0$ of (8.6.1) is integrally asymptotically stable.

Proof. At first, let us prove item (i). Suppose the trivial solution $y^{*} \equiv 0$ of (8.5.1) is integrally stable. Also, assume that for every $\varepsilon>0$, there is $\delta=\delta(\varepsilon)>0$ such that $\phi:[\gamma-r, \gamma] \rightarrow \mathbb{R}^{n}$ with $\|\phi\|_{\infty}<\delta$ and

$$
\sup _{t \in[\gamma, v]_{\mathbb{T}}}\left|\int_{\gamma}^{t} p(s) \Delta s\right|<\delta
$$


where $t_{0} \leq \gamma<v<\infty$, with $\gamma, v \in \mathbb{T}$. By definition, we obtain

$$
\|\phi\|_{\infty}=\sup _{t \in[\gamma-r, \gamma]_{\mathbb{T}}}|\phi(t)|<\delta
$$

Also, since $\gamma \in \mathbb{T}$, then, by the definition, for every $t \in[\gamma-r, \gamma]$, we have $t^{*} \in[\gamma-r, \gamma]_{\mathbb{T}}$. Then, combining this fact and the definition of extension of $\phi$, we obtain

$$
\left\|\phi_{\gamma}^{*}\right\|_{\infty}<\delta
$$

By Theorem 4.12, we have

$$
\int_{\gamma}^{t} p(s) \mathrm{d} u(s)=\int_{\gamma}^{t} p^{*}(s) \mathrm{d} u(s)
$$

for every $t \in[\gamma, v]$. On the other hand, if we define

$$
F(t)=\int_{\gamma}^{t} p^{*}(s) \mathrm{d} u(s), t \in[\gamma, v]
$$

then, by Lemma 4.21, we have

$$
F(t)=\int_{\gamma}^{t} p^{*}(s) \mathrm{d} u(s)=\int_{\gamma}^{t^{*}} p^{*}(s) \mathrm{d} u(s) .
$$

Also, since $t^{*} \in \mathbb{T}$, it follows from Theorem 4.22 that the last integral coincides with $\int_{\gamma}^{t} p(s) \Delta s$, which means that $\sup _{t \in[\gamma, v]}\|F(t)\|$ must be the same as $\sup _{t \in[\gamma, v]_{\mathbb{T}}}\left|\int_{\gamma}^{t} p(s) \Delta s\right|$. Thus, inequality (8.6.3) implies that

$$
\sup _{t \in[\gamma, v]}\left|\int_{\gamma}^{t} p(s) \mathrm{d} u(s)\right|<\delta
$$

Now, since $y^{*} \equiv 0$ is integrally stable, by definition and by the above equations, we obtain

$$
\left|\bar{y}_{t}^{*}\left(\gamma, \phi_{\gamma}^{*}\right)\right|<\varepsilon, \text { for every } t \in[\gamma, v]
$$

where $\bar{y}^{*}\left(t ; \gamma, \phi_{\gamma}^{*}\right)$ is a solution of the perturbed equation

$$
\bar{y}^{*}(t)=\bar{y}^{*}(\gamma)+\int_{\gamma}^{t} f\left(\bar{y}_{s}^{*}, s\right) \mathrm{d} g(s)+\int_{\gamma}^{t} p(s) \mathrm{d} u(s)+\sum_{\substack{k \in\{1, \ldots, m\}, t_{k}<t}} I_{k}\left(\bar{y}^{*}\left(t_{k}\right)\right),
$$


such that $\bar{y}_{\gamma}=\phi_{\gamma}^{*}$. Then, we obtain

$$
\left|\bar{y}_{t}(\gamma, \phi)\right|<\varepsilon, \text { for every } t \in[\gamma, v]_{\mathbb{T}}
$$

where $\bar{y}(t ; \gamma, \phi)$ is a solution of the perturbed functional dynamic equation with impulses

$$
\bar{y}(t)=\bar{y}(\gamma)+\int_{\gamma}^{t} f\left(\bar{y}_{s}, s\right) \Delta s+\int_{\gamma}^{t} p(s) \Delta s+\sum_{\substack{k \in\{1, \ldots, m\}, t_{k}<t}} I_{k}\left(\bar{y}\left(t_{k}\right)\right),
$$

such that $\bar{y}(t)=\phi(t)$, for $t \in[\gamma-r, \gamma]_{\mathbb{T}}$, by Theorem 4.23. Thus, by the definition, it follows that the solution $y \equiv 0$ of (8.6.1) is integrally stable.

Reciprocally, suppose the solution $y \equiv 0$ of (8.6.1) is integrally stable. Also, assume that for every $\varepsilon>0$, there is $\delta=\delta(\varepsilon)>0$ such that $\phi_{\gamma}^{*} \in P$ with $\left\|\phi_{\gamma}^{*}\right\|_{\infty}<\delta$ and

$$
\sup _{t \in[\gamma, v]}\left|\int_{\gamma}^{t} p(s) \mathrm{d} u(s)\right|<\delta \text {. }
$$

Thus, it follows immediately that

$$
\|\phi\|<\delta
$$

since $\phi$ is a restriction of the function $\phi_{\gamma}^{*}$. Again, by Theorem 4.12, we obtain

$$
\int_{\gamma}^{t} p(s) \mathrm{d} u(s)=\int_{\gamma}^{t} p^{*}(s) \mathrm{d} u(s)
$$

for every $t \in[\gamma, v]$. Moreover, by Lemma 4.21, we have

$$
\int_{\gamma}^{t} p^{*}(s) \mathrm{d} u(s)=\int_{\gamma}^{t^{*}} p^{*}(s) \mathrm{d} u(s)
$$

and using the fact that $t^{*} \in \mathbb{T}$, we obtain, by Theorem 4.22 and by inequality (8.6.4),

$$
\sup _{t \in[\gamma, v]_{\mathbb{T}}}\left|\int_{\gamma}^{t} p(s) \Delta s\right|<\delta
$$

Since $y \equiv 0$ is integrally stable, by definition, we have

$$
\left|\bar{y}_{t}(\gamma, \phi)\right|<\varepsilon, \text { for every } t \in[\gamma, v]_{\mathbb{T}}
$$


where $\bar{y}(t ; \gamma, \phi)$ is a solution of the impulsive perturbed dynamic equation

$$
\bar{y}(t)=\bar{y}(\gamma)+\int_{\gamma}^{t} f\left(\bar{y}_{s}, s\right) \Delta s+\int_{\gamma}^{t} p(s) \Delta s+\sum_{\substack{k \in\{1, \ldots, m\}, t_{k}<t}} I_{k}\left(\bar{y}\left(t_{k}\right)\right),
$$

satisfying $\bar{y}(t)=\phi(t)$, for $t \in[\gamma-r, \gamma]_{\mathbb{T}}$ Then, by Theorem 4.23 and by the definition of $\bar{y}^{*}$, we obtain

$$
\left|\bar{y}_{t}^{*}\left(\gamma, \phi_{\gamma}^{*}\right)\right|<\varepsilon, \text { for every } t \in[\gamma, v],
$$

since $\gamma, v \in \mathbb{T}$, where $\bar{y}^{*}\left(t ; \gamma, \phi_{\gamma}^{*}\right)$ is a solution of the perturbed equation

$$
\bar{y}^{*}(t)=\bar{y}^{*}(\gamma)+\int_{\gamma}^{t} f\left(\bar{y}_{s}^{*}, s^{*}\right) \mathrm{d} g(s)+\int_{\gamma}^{t} p(s) \mathrm{d} u(s)+\sum_{\substack{k \in\{1, \ldots, m\}, t_{k}<t}} I_{k}\left(\bar{y}^{*}\left(t_{k}\right)\right),
$$

such that $\bar{y}_{\gamma}^{*}=\phi_{\gamma}^{*}$ for $t \in[-r, 0]$. Then, by the definition, it follows that the solution $y^{*} \equiv 0$ of (8.5.1) is integrally stable.

Suppose the solution $y^{*} \equiv 0$ of (8.5.1) is integrally attracting and assume that there is a $\widetilde{\delta}>0$ and for every $\varepsilon>0$, there exist $T=T(\varepsilon) \geq 0$ and $\rho=\rho(\varepsilon)>0$ such that $\|\phi\|<\widetilde{\delta}$ and

$$
\sup _{t \in\left[\gamma, t_{1}\right]_{\mathbb{T}}}\left|\int_{\gamma}^{t} p(s) \Delta s\right|<\rho .
$$

Then, using the same arguments as before, we obtain

$$
\sup _{t \in[\gamma, v]}\left|\int_{\gamma}^{t} p(s) \mathrm{d} u(s)\right|<\rho \quad \text { and } \quad\left\|\phi_{\gamma}^{*}\right\|<\widetilde{\delta} .
$$

Since $y^{*} \equiv 0$ is regularly attracting, then

$$
\left|\bar{y}_{t}^{*}\left(\gamma, \phi_{\gamma}^{*}\right)\right|<\varepsilon, \text { for all } t \geq \gamma+T, t \in[\gamma, v]
$$

where $\bar{y}^{*}\left(t ; \gamma, \phi_{\gamma}^{*}\right)$ is a solution of the impulsive measure perturbed differential equation

$$
\bar{y}^{*}(t)=\bar{y}^{*}(\gamma)+\int_{\gamma}^{t} f\left(\bar{y}_{s}^{*}, s\right) \mathrm{d} g(s)+\int_{\gamma}^{t} p(s) \mathrm{d} h(s)+\sum_{\substack{k \in\{1, \ldots, m\}, t_{k}<t}} I_{k}\left(\bar{y}^{*}\left(t_{k}\right)\right),
$$

satisfying $\bar{y}_{\gamma}^{*}=\phi_{\gamma}^{*}$ for $t \in[-r, 0]$. Then, since $\bar{y}$ is a restriction of $\bar{y}^{*}$, we obtain

$$
|\bar{y}(t ; \gamma, \phi)|<\varepsilon, \text { for all } t \geq \gamma+T, t \in[\gamma, v]_{\mathbb{T}},
$$


where $\bar{y}(t ; \gamma, \phi)$ is a solution of the perturbed dynamic equation with impulses

$$
\bar{y}(t)=\bar{y}(\gamma)+\int_{\gamma}^{t} f\left(\bar{y}_{s}, s\right) \Delta s+\int_{\gamma}^{t} p(s) \Delta s+\sum_{\substack{k \in\{1, \ldots, m\}, t_{k}<t}} I_{k}\left(\bar{y}\left(t_{k}\right)\right),
$$

such that $\bar{y}(t)=\phi(t)$, for $t \in[\gamma-r, \gamma]_{\mathbb{T}}$, by Theorem 4.23. Thus, by the definition, it follows that the solution $y \equiv 0$ of (8.6.1) is integrally attracting.

Reciprocally, suppose the solution $y \equiv 0$ of (8.6.1) is integrally attracting and assume that there is $\widetilde{\delta}>0$ and for every $\varepsilon>0$, there exist $T=T(\varepsilon) \geq 0$ and $\rho=\rho(\varepsilon)>0$ such that if $\left\|\phi_{\gamma}^{*}\right\|_{\infty}<\widetilde{\delta}, \phi_{\gamma}^{*} \in P$ and

$$
\sup _{t \in[\gamma, v]}\left|\int_{\gamma}^{t} p(s) \mathrm{d} u(s)\right|<\rho
$$

where $t_{0} \leq \gamma<v<\infty$ with $\gamma, v \in \mathbb{T}$. Also, by the same argument as before, we have

$$
\sup _{t \in[\gamma, v]_{\mathbb{T}}}\left|\int_{\gamma}^{t} p(s) \Delta s\right|<\rho
$$

Moreover, since $\phi$ is a restriction of $\phi_{\gamma}^{*}$, we obtain that

$$
\|\phi\|_{\infty}<\widetilde{\delta}
$$

Since $y \equiv 0$ is integrally attracting, then, by definition and above inequalities, we obtain

$$
\left|\bar{y}_{t}(\gamma, \phi)\right|<\varepsilon, \text { for all } t \geq \gamma+T, t \in[\gamma, v]_{\mathbb{T}}
$$

where $\bar{y}(t ; \gamma, \phi)$ is a solution of the perturbed functional dynamic equation with impulses

$$
\bar{y}(t)=\bar{y}(\gamma)+\int_{\gamma}^{t} f\left(\bar{y}_{s}, s\right) \Delta s+\int_{\gamma}^{t} p(s) \Delta s+\sum_{\substack{k \in\{1, \ldots, m\}, t_{k}<t}} I_{k}\left(\bar{y}\left(t_{k}\right)\right),
$$

satisfying $\bar{y}(t)=\phi(t)$, for $t \in[\gamma-r, \gamma]_{\mathbb{T}}$. Then, using the same argument as before, we obtain

$$
\left|\bar{y}_{t}^{*}\left(\gamma, \phi_{\gamma}^{*}\right)\right|<\varepsilon, \text { for all } t \geq \gamma+T, t \in[\gamma, v]
$$

where $\bar{y}^{*}\left(t ; \gamma, \phi_{\gamma}^{*}\right)$ is a solution of the impulsive measure perturbed functional differential 
equation

$$
\bar{y}^{*}(t)=\bar{y}^{*}(\gamma)+\int_{\gamma}^{t} f\left(\bar{y}_{s}^{*}, s\right) \mathrm{d} g(s)+\int_{\gamma}^{t} p(s) \mathrm{d} u(s)+\sum_{\substack{k \in\{1, \ldots, m\}, t_{k}<t}} I_{k}\left(\bar{y}^{*}\left(t_{k}\right)\right),
$$

satisfying $\bar{y}_{\gamma}^{*}(t)=\phi_{\gamma}^{*}(t)$, for $t \in[-r, 0]_{\mathbb{T}}$, by Theorem 4.23. Thus, by the definition, it follows that the solution $y^{*} \equiv 0$ of (8.5.1) is integrally attracting.

The item (iii) follows immediately combining items (i) and (ii).

Combining the previous theorem, Theorem 4.23, Corollary 8.20 and Lemma 4.24, we obtain immediately the next result which shows some relations between the stability concepts for impulsive functional dynamic equations on time scales and the corresponding ones for generalized ODEs.

Corollary 8.25. Suppose the function $f: P \times\left[t_{0}, t_{0}+\eta\right]_{\mathbb{T}} \rightarrow \mathbb{R}^{n}$ satisfies the following conditions:

1. The integral $\int_{t_{0}}^{t_{0}+\eta} f\left(y_{t}, t\right) \Delta t$ exists for every $y \in O$.

2. There exists a constant $M>0$ such that

$$
\left|\int_{u_{1}}^{u_{2}} f\left(y_{t}, t\right) \Delta t\right| \leq M\left(u_{2}-u_{1}\right)
$$

for every $y \in O$ and $u_{1}, u_{2} \in\left[t_{0}, t_{0}+\eta\right]_{\mathbb{T}}, u_{1} \leq u_{2}$.

3. Assume there exists a constant $L>0$ such that

$$
\left|\int_{u_{1}}^{u_{2}}\left(f\left(y_{t}, t\right)-f\left(z_{t}, t\right)\right) \Delta t\right| \leq L \int_{u_{1}}^{u_{2}}\left\|y_{t}-z_{t}\right\|_{\infty} \Delta t
$$

for every $y, z \in O$ and $u_{1}, u_{2} \in\left[t_{0}, t_{0}+\eta\right]_{\mathbb{T}}, u_{1} \leq u_{2}$.

Also, suppose the function $p:\left[t_{0}, t_{0}+\eta\right]_{\mathbb{T}} \rightarrow \mathbb{R}^{n}$ satisfies the following conditions:

1. The integral $\int_{t_{0}}^{t_{0}+\eta} p(t) \Delta t$ exists.

2. There exists a constant $M_{1}>0$ such that

$$
\left|\int_{u_{1}}^{u_{2}} p(t) \Delta t\right| \leq M_{1}\left(u_{2}-u_{1}\right)
$$

for every $u_{1}, u_{2} \in\left[t_{0}, t_{0}+\eta\right]_{\mathbb{T}}, u_{1} \leq u_{2}$. 
Further, let $t_{0} \in \mathbb{T}$ and assume that for every $y \in G\left(\left[t_{0}-r, t_{0}+\eta\right]_{\mathbb{T}}, B\right)$, where $B \subset \mathbb{R}^{n}$ open, the function $t \mapsto f\left(y_{t}^{*}, t\right)$ is Kurzweil-Henstock delta-integrable on $\left[t_{0}, t_{0}+\eta\right]_{\mathbb{T}}$. Moreover, suppose the function $t \mapsto p(t)$ is Kurzweil-Henstock delta-integrable on $\left[t_{0}, t_{0}+\eta\right]_{\mathbb{T}}$. Define the functions $g(s)=s^{*}$ and $h(s)=s^{*}$ for every $s \in\left[t_{0}, t_{0}+\eta\right]$. Suppose, for each $k$, the impulses operators $I_{k}: \mathbb{R}^{n} \rightarrow \mathbb{R}^{n}$ satisfy the conditions

$\left(H_{6}\right)$ There exists a constant $K_{1}>0$ such that

$$
\left|I_{k}(x)\right| \leq K_{1}
$$

for every $k \in\{1, \ldots, m\}$ and $x \in B$.

$\left(H_{7}\right)$ There exists a constant $K_{2}>0$ such that

$$
\left|I_{k}(x)-I_{k}(y)\right| \leq K_{2}|x-y|
$$

for every $k \in\{1, \ldots, m\}$ and $x, y \in B$.

Then, the following statements hold:

(i) The trivial solution $y \equiv 0$ of (8.6.1) is integrally stable, if and only if, the trivial solution $x \equiv 0$ of (8.4.3) is regularly stable.

(ii) If the trivial solution $x \equiv 0$ of (8.4.3) is regularly attracting, then the trivial solution $y \equiv 0$ of (8.6.1) is integrally attracting.

(iii) If the trivial solution $x \equiv 0$ of (8.4.3) is regularly asymptotically stable, then the trivial solution $y \equiv 0$ of (8.6.1) is integrally asymptotically stable. 


\section{- \\ Open and developing problems}

As it was explained before, our goal in the present work was to develop the theory of impulsive functional dynamic equations on time scales. In order to do it, we started by establishing a correspondence between the solutions of an impulsive functional dynamic equation on time scales and the solutions of an impulsive measure functional differential equation and, also, a correspondence between those and the solutions of a generalized ordinary differential equation.

These correspondences allowed us to obtain several interesting and new results for all these equations just using results for generalized ordinary differential equations. In this way, these correspondences facilitate the obtainment of good results for impulsive functional dynamic equations on time scales and impulsive measure functional differential equations, since the framework of abstract generalized ordinary differential equations is much simpler to deal with than any of the above specific setting of differential equations.

Thus, in this direction, we were able to prove several results for impulsive functional dynamic equations on time scales and impulsive measure functional differential equations, such as local existence and uniqueness, continuous dependence of solutions on the initial data, periodic and non-periodic averaging principles and, also, stability results. These results improve the literature concerning these equations, since they have been studied recently and the literature concerning them is still scarce. See, for instance, [19] and [37].

Although we have obtained several new results for impulsive functional dynamic equations on time scales and impulsive measure functional differential equations, the research in this direction is far beyond being finished and complete. For instance, we mention 
Converse Lyapunov theorems for these equations. One idea to obtain these results is to improve the known results concerning Lyapunov theorems for generalized ordinary differential equations (see [2], for instance), using the stability concepts for these equations introduced in Chapter 8, and then, to translate them to impulsive functional dynamic equations on time scales and to measure functional differential equations with impulses, using the correspondences, obtaining good results for them. This work is being developed by M. Federson, J. G. Mesquita and E. Toon in [39].

Another open problem is to prove results concerning periodic and almost periodic solutions of impulsive measure functional differential equations and impulsive functional dynamic equations on time scales. The idea, in this case, would be to prove results on periodic and almost periodic solutions for generalized differential equations, and then, to translate them to impulsive functional dynamic equations on time scales and to measure functional differential equations with impulses, using the correspondences.

We can mention, also, one more developing problem whose goal is to establish a correspondence between generalized ordinary differential equations and neutral measure functional differential equations in order to obtain interesting and new results for these last ones. This is motivated because while the theory concerning neutral functional differential equations is extensive (see [11],[22], [55], [57], [58], [59], for instance), the literature concerning neutral measure functional differential equations is really scarce. As a matter of fact, we do not know any result in this direction yet. We point out that these last equations generalize neutral functional differential equations. See [57], for instance. In this direction, M. Federson, M. Frasson, J. G. Mesquita and P. Tacuri are developing some results. See [33].

Furthermore, we can continue the development of the previous work, by obtaining a correspondence between neutral functional dynamic equations on time scales and neutral measure functional differential equations. Using such correspondence, it will be possible to establish an indirect correspondence between neutral functional dynamic equations on time scales and generalized differential equations, using [33], and in this way, it will be possible to develop several new results for these equations, just by translating known results from generalized ordinary differential equations. This is an interesting area to explore and there are several results to be developed, since neutral functional dynamic equations on time scales are really new in the literature. See [23], [47] and [91], for instance.

In conclusion, the theory of generalized ordinary differential equations is a powerful tool to develop results for several types of equations, since generalized ordinary differential 
equations encompass most of them. See [3], [36], [40], [100], for instance. Also, it turns out that the framework of abstract generalized ordinary differential equations is simpler to deal with than any of the above specific setting of differential equations.

On the other hand, there are other types of equations which are not related to generalized ordinary differential equations yet. We can mention as examples partial differential equations, stochastic equations and, also, fractional differential equations. The figure below illustrates some inclusions.

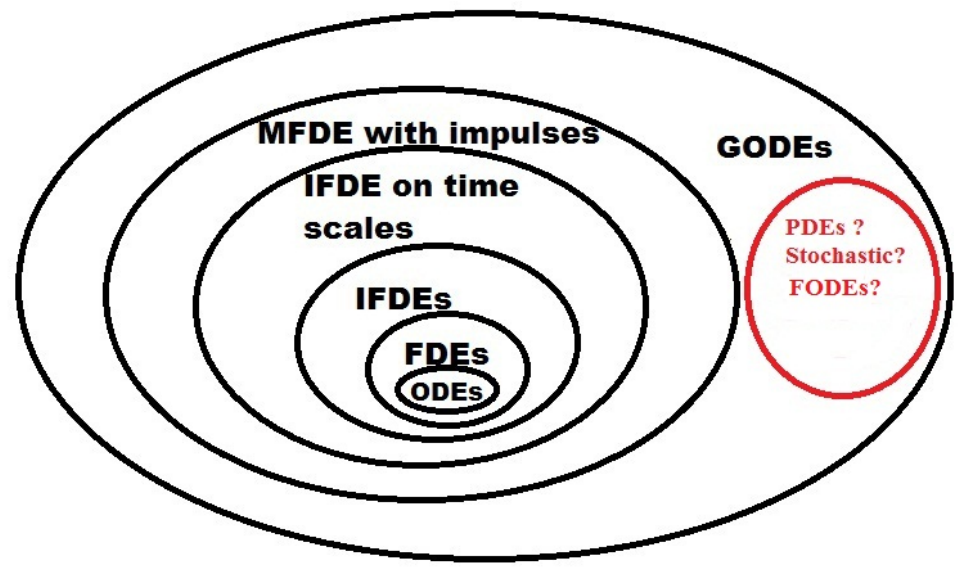

These questions certainly offer several opportunities to develop future and interesting projects, since by using the relations between generalized ordinary differential equations and other types of equations, one is able to reduce a given problem by establishing it in the setting of a result for generalized ordinary differential equations and then to translate the results to other types of equations. 



\section{Bibliography}

[1] Ladislav Adamec, A note on continuous dependence of solutions of dynamic equations on time scales, J. Difference Equ. Appl., 17 (2011), no 5, 647-656.

[2] S. M. Afonso, E. M. Bonotto, M. Federson, L. P. Gimenes, Boundedness of solutions of functional differential equations with variable impulses via generalized ordinary differential equations, Mathematische Nachrichten, v. 285, p. 545-561, 2012.

[3] S. M. Afonso, E. Bonotto, M. Federson, L. P. Gimenes, Stability of functional differential equations with variable impulsive perturbations via generalized ordinary differential equations, Bulletin des Sciences Mathématiques, (Paris 1885), to appear.

[4] S. M. Afonso, E. M. Bonotto, M. Federson, Š Schwabik, Discontinuous local semiflows for Kurzweil equations leading to LaSalle's Invariance Principle for differential systems with impulses at variable times, Journal of Differential Equations, vol. 250 (2011), 2969-3001.

[5] Z. Artstein, Averaging of time-varying differential equations revisited. J. Differential Equations 243 (2007), no. 2, 146-167.

[6] Ferhan M. Atici, Daniel C. Biles, Alex Lebedinsky, An application of time scales to economics, Mathematical and Computer Modelling, 43, 718-726 (2006).

[7] D. D. Bainov, S. D. Milusheva, Application of the averaging method for functional differential equations with impulses. J. Math. Anal. Appl. 95 (1983), 85-105.

[8] D. D. Bainov, S. D. Milusheva, Justification of the averaging method for a system of functional differential equations with variable structure and impulses, Appl. Math. and Optimization, 16 (1987), 19-36. 
[9] D. Bainov, S. D. Milusheva, Justification of the averaging method for a class of functional differential equations with impulses, J. London Math. Soc. 25(2) (1982), 309-331.

[10] S. S. Bellale, C. B. Dhage, Existence theorems for perturbed abstract measure differential equations, Nonlinear Anal. 71 (2009), no. 12, e319-e328.

[11] R. Bellman and K. Cooke, Differential-difference equations, Academic Press, New York, 1963.

[12] M. Benchohra, J. Henderson, S. K. Ntouyas, and A. Ouahab, Impulsive functional dynamic equations on time scales, Dynamic Systems and Applications, 15 (2006), $43-52$.

[13] M. Benchohra, J. Henderson, S. K. Ntouyas, and A. Ouahab, On First Order Impulsive Dynamic Equations on Time Scales, Journal of Difference Equations and Applications, vol. 10, no. 6 (2004), 541-548.

[14] N. N. Bogolyubov, N. N. Krylov, New Methods in Linear Mechanics, Kiev, GTTs, 1934 (Russian).

[15] N. N. Bogolyubov, A. Mitropolskii, Assymptotic Methods in the Theory of Non-linear Oscillations, Moscou, Nauka, 1963 (Russian).

[16] M. Bohner, M. Federson, J. G. Mesquita, Continuous dependence for impulsive functional dynamic equations involving variable time scales, submitted.

[17] M. Bohner and A. Peterson, Dynamic Equations on Time Scales: An Introduction with Applications, Birkhäuser, Boston, 2001.

[18] M. Bohner and A. Peterson, Advances in Dynamic Equations on Time Scales, Birkhäuser, Boston, 2003.

[19] M. H. Chang, P. T. Liu, Maximum principles for control systems described by measure functional differential equations, J. Optimization Theory Appl. 15 (1975), 517531.

[20] Y. Chang, W. Li, Existence results for impulsive dynamic equations on time scales with nonlocal initial conditions, Mathematical and Computer Modelling, 43 (2006), $377-384$. 
[21] D. N. Chate, B. C. Dhage, S. K. Ntouyas, Abstract measure differential equations, Dynam. Systems Appl., 13 (2004), no. 1, 105-117.

[22] M. A. Cruz, J. K. Hale, Asymptotic behavior of neutral functional differential equations, Arch. Rational Mech. Anal., Vol. 34, (1969), 331-353.

[23] J. Dai, Y. Li, T. Zhang, Positive periodic solutions for nonautonomous impulsive neutral functional differential systems with time-varying delays on time scales, Electron. J. Differential Equations, 2010, No. 34, 10 pp.

[24] P. C. Das, R. R. Sharma, Existence and stability of measure differential equations, Czech. Math. Journal, 22 (97) (1972), 145-158.

[25] S. G. Deo and S. R. Joshi, On abstract measure delay differential equations, An. Stiint. Univ. Al. I. Cuza Iasi, N. Ser., Sect. Ia 26, 327-335 (1980).

[26] S. G. Deo and S. G. Pandit, Differential systems involving impulses, Lecture Notes in Mathematics, vol. 954, Springer-Verlag, Berlin, 1982.

[27] B. C. Dhage, On abstract measure integro-differential equations, J. Math. Phy. Sci. 20 (1986), 367-380.

[28] B. C. Dhage, On system of abstract measure integro-differential inequalities and applications, Bull. Inst. Math. Acad. Sinica, 18 (1989), 65-75.

[29] B. C. Dhage, Mixed monotonicity theorems for a system of abstract measure delay integro-differential equations, An. Stint. Univ. "Al. I. Cuza" Iasi, XLII (1996), 355366.

[30] B. C. Dhage, R. John Graef, On stability of abstract measure delay integrodifferential equations, Dynam. Systems Appl. 19 (2010), no. 2, 323-334.

[31] Norah Esty and Stefan Hilger, Convergence of time scales under the Fell topology, J. Difference Equ. Appl., 15:10, (2009) 1011-1020.

[32] M. Federson, D. Fraňková, J. G. Mesquita, Compactness of $G([a, b], X)$ and continuous dependence results for various types of equations, submitted.

[33] M. Federson, M. Frasson, J. G. Mesquita, P. Tacuri, Neutral measure functional differential equations, pre-print. 
[34] M. Federson, J. G. Mesquita, Non-periodic averaging principles for measure functional differential equations and functional dynamic equations on time scales involving impulses, submitted.

[35] M. Federson, J. G. Mesquita, Averaging for functional retarded differential equations, J. Math. Anal. Appl., v. 382, p. 77-85, 2011.

[36] M. Federson, J. G. Mesquita, A. Slavík, Measure functional differential equations and functional dynamic equations on time scales, J. Diff. Equations, 252 (2012), 38163847.

[37] M. Federson, J. G. Mesquita, A. Slavík, Basic results for functional differential and dynamic equations involving impulses, Mathematische Nachrichten, to appear.

[38] M. Federson, J. G. Mesquita, E. Toon, Stability results for measure functional differential equations and functional dynamic equations on time scales involving impulses, submitted.

[39] M. Federson, J. G. Mesquita, E. Toon, Lyapunov theorems for measure functional differential equations and functional dynamic equations on time scales involving impulses, in preparation.

[40] M. Federson, Š. Schwabik, Generalized ODE approach to impulsive retarded functional differential equations, Differential and Integral Equations, 19 (11), (2006), 1201-1234.

[41] M. Federson; Š. Schwabik, A new approach to impulsive retarded differential equations: stability results, Functional Differential Equations, 16(4) (2009), 583-607.

[42] M. Federson, Š. Schwabik, Stability for retarded functional differential equations, Ukrainian Math J., 60 (1), 2008, 107-126.

[43] M. Federson, P. Táboas, Topological dynamics of retarded functional differential equations, J. Differential Equations 195, no. 2 (2003), 313-331.

[44] V. I. Fodčuk, The method of averaging for differential difference equations of the neutral type, Ukrainian Math. J., 20 (1968), 203-209.

[45] D. Fraňková, Continuous dependence on a parameter of solutions of generalized differential equations, Časopis pro pěstováni matematiky, Vol. 114 (1989), No. 3, 230-261.

[46] D. Fraňková, Regulated functions, Mathematica Bohemica, No. 1, 20-59, 116 (1991). 
[47] C. Gao, T. Li, S. Tang, E. Thandapani, Oscillation theorems for second-order neutral functional dynamic equations on time scales, Electron. J. Differential Equations, 2011, No. 101, 9 pp.

[48] J. B. Godoy, Método da média para Equações diferenciais funcionais retardadas impulsivas via equações diferenciais generalizadas, Dissertação de Mestrado, ICMCUSP, 2009.

[49] R. A. Gordon, The Integrals of Lebesgue, Denjoy, Perron, and Henstock, American Mathematical Society, 1994.

[50] J. R. Graef, A. Ouahab, Nonresonance Impulsive Functional Dynamic Equations on Times Scales, International Journal of Applied Mathematical Sciences, vol. 2, no. 1 (2005), 65-80.

[51] I. Györi, Numerical approximation of the solutions of delay differential equations on an infinite interval using piecewise constant arguments, Comput. Math. Appl., 28 (1994), no 1-3, 81-92.

[52] A. Halanay, Differential Equations: Stability, Oscillations, Time Lags. Academic Press, New York, 1966.

[53] A. Halanay, On the method of averaging for differential equations with retarded argument, J. Math. Anal. Appl. 14 (1966), 70-76.

[54] J. K. Hale, Averaging methods for differential equations with retarded arguments with a small parameter, J. Differential Equations, 2 (1966), 57-73.

[55] J. K. Hale, A class of neutral equations with the fixed point property, Proc. Nat. Acad. Sci. U.S.A., Vol. 67, (1970), 136-137.

[56] J. K. Hale; S. M. Verduyn Lunel, Averaging in infinite dimensions, J. Integral Equations Appl. 2(4) (1990), 463-494.

[57] J. K. Hale, S. M. V. Lunel, Introduction to Functional Differential Equations, Springer-Verlag, New York, 1993.

[58] J. K. Hale, K. R. Meyer, A class of functional equations of neutral type, Memoirs of the American Mathematical Society, No. 76, 1967.

[59] D. Henry, Linear autonomous neutral functional differential equations, J. Differential Equations., 15, (1974), 106-128. 
[60] R. Henstock, Lectures on the Theory of Integration, World Scientific, Singapore, 1988.

[61] S. Hilger, Analysis on measure chains - a unified approach to continuous and discrete calculus, Results Math. 18, 18-56 (1990).

[62] S. Hilger, Ein Maßkettenkalkül mit Anwendung auf Zentrumsmanningfaltigkeiten, PhD thesis, Universität Würzburg, 1988.

[63] J. Hoffacker, C. C. Tisdell, Stability and Instability for dynamic equations on time scales, Computers and Mathematics with Applications, 2005.

[64] C. S. Hönig, Volterra Stieltjes Integral Equations, North Holland Publ. Comp., Amsterdam, 1975.

[65] C. S. Hönig, As integrais de gauge (de Kurzweil, de Henstock e de McShane), Minicurso do 37o Seminário Brasileiro de Análise, 1993, p. 1 - 60, São Paulo.

[66] A. Huseynov, Riesz representation theorem on time scales, Mathematical and Computer Modelling (2011), doi:10.1016/j.mcm.2011.10.055.

[67] C. Imaz, Z. Vorel, Generalized ordinary differential equations in Banach spaces and applications to functional equations, Bol. Soc. Mat. Mexicana, 11 (1966), 47-59.

[68] S. R. Joshi, A system of abstract measure delay differential equations, J. Math. Phys. Sci. 13 (1979), 497-506.

[69] S. R. Joshi, G. R. Shendge, Abstract measure differential inequalities and applications, Acta Math. Hung., 41 (1983), 53-54.

[70] B. Karpuz, Existence and uniqueness of solutions to systems of delay dynamic equations on time scales, Int. J. Math. Comput., vol. 10, no. M11 (2011), 48-58.

[71] V. Kolmanovskii and A. Myshkis, Applied Theory of Functional Differential Equations, Kluwer Academic Publishers, Vol 85, 1992.

[72] J. Kurzweil, Generalized ordinary differential equations and continuous dependence on a parameter, Czech. Math. J., 7 (82), (1957), 418-448.

[73] J. Kurzweil, Generalized ordinary differential equations, Czechoslovak Math. J. 8(83) (1958), 360-388.

[74] M. Lakrib, Time averaging for functional differential equations, J. Appl. Math. 1, (2003), 1-16. 
[75] M. Lakrib, T. Sari, Averaging results for functional differential equations, Siberian Math. J. 45(2), (2004), 311-320.

[76] Bonita A. Lawrence and Ralph W. Oberste-Vorth, Solutions of dynamic equations varying time scales, Difference equations, special functions and orthogonal polynomials, 452-461, World Sci. Publ., Hackensack, NJ, 2007.

[77] B. Lehman, The influence of delays when averaging slow and fast oscillating systems: overview. Special issue on analysis and design of delay and propagation systems. IMA J. Math. Control Inform. 19(1-2), (2002), 201 - 215.

[78] B. Lehman; S. P. Weibel, Averaging theory for delay difference equations with timevarying delays. SIAM J. Appl. Math. 59(4), (1999), 1487 - 1506.

[79] B. Lehman, S. P. Weibel, Fundamental theorems of averaging for functionaldifferential equations, J. Differential Equations 152(1), (1999), 160 - 190.

[80] B. Lehman, S. P. Weibel, Averaging theory for functional differential equations, Proc. 37th IEEE Conference on Decision and Control, USA, (1998), 1352 - 1357.

[81] X. Liu, W. Wang, J. Wu, Delay dynamic equations on time scales, Appl. Anal. 89 (2010), no. 8, $1241-1249$.

[82] G. N. Medvedev, Asymptotic solutions of some systems of differential equations with deviating argument, Dokl. Akad. Nauk, 9 (1968), 85-87.

[83] G. N. Medvedev; B. I. Morgunov, V. M. Volosov, Application of the averaging method to the solution of certain systems of differential equations with deviating argument. (Russian) Vestnik Moskov. Univ. Ser. III Fiz. Astronom 1965(6) (1965), 89-91.

[84] J. G. Mesquita, A. Slavík, Periodic averaging theorems for various types of equations, J. Math. Anal. Appl., 387 (2012), 862-877.

[85] A. B. Mingarelli, Volterra-Stieltjes Integral Equations and Generalized Ordinary Equations Expressions, Springer-Verlag, Berlin Heidelberg, New York, Tokyo, 1983.

[86] G. A. Monteiro and M. Tvrdý, Generalized linear differential equations in a Banach space: Continuous dependence on a parameter, Discrete Contin. Dyn. Syst., 2012 (to appear).

[87] D. Mozyrska, E. Pawluszewicz, D. F. M. Torres, The Riemann-Stieltjes integral on time scales, Aust. J. Math. Anal. Appl. 7 (2010), 1-14. 
[88] F. Oliva, Z. Vorel, Functional equations and generalized ordinary differential equations, Bol. Soc. Mat. Mexicana, 11 (1966), 40-46.

[89] N. Onuchic, Equações diferenciais com retardamento, Biblioteca de autores e publicações brasileiras de matemática, ICMC - Universidade de São Paulo, São Carlos, 1971.

[90] A. Peterson, B. Thompson, Henstock-Kurzweil delta and nabla integrals, J. Math. Anal. Appl., 323 (2006), 162- 178.

[91] S. H. Saker, Oscillation criteria for second-order quasilinear neutral functional dynamic equation on time scales, Neliniini-Koliv 13, (2010), no 3, 379-399, translation in Nonlinear Oscil. (N. Y.) 13 (2011), no 3, 407-428.

[92] J. A. Sanders and F. Verhulst, Averaging methods in Nonlinear Dynamical Systems, Applied Mathematical Sciences 59, Springer-Verlag, 1983

[93] J. A. Sanders, F. Verhulst, and J. Murdock, Averaging Methods in Nonlinear Dynamical Systems (2nd edition), Springer, New York, 2007.

[94] Š. Schwabik, Generalized Ordinary Differential Equations, Series in Real Anal., vol. 5, World Scientific, Singapore, 1992.

[95] ك̌. Schwabik, Variational stability for generalized ordinary differential equations, $\check{C} a$ sopis Pěst. Mat. 109(4), (1984), 389-420.

[96] R. R. Sharma, An abstract measure differential equation, Proc. Amer. Math. Soc. 32 (1972), 503-510.

[97] R. R. Sharma, A measure differential inequality with applications, Proc. Amer. Math. Soc. 48 (1975), 87-97.

[98] A. Slavík, Dynamic equations on time scales and generalized ordinary differential equations, J. Math. Anal. Appl. 385 (2012), 534-550.

[99] A. Slavík, Averaging dynamic equations on time scales, J. Math. Anal. and Appl., 388, 996-1012, 2012.

[100] A. Slavík, Measure functional differential equations with infinite delay, submitted.

[101] V. Strygin, The averaging principle for equations with hereditary. Ukrainian Math. J., 22(4), (1971), 430-439. 
[102] F. Verhulst, Nonlinear Differential Equations and Dynamical Systems (2nd edition), Springer, 2000. 


\section{Index}

Averaging Principles, 131

Backward jump operator, 34

Banach space, 17, 105

Conditions

Boundedness, 79

Carathéodory, 79, 87

Lipschitz, 79, 87

Correspondence, 59

Delta integral, 44

Lower Darboux, 42

Upper Darboux, 42

Delta-derivative, 37, 38

Delta-gauge, 48

Delta-integral

Darboux, 42, 43

Indefinite, 46

Kurzweil-Henstock, 48, 82, 84

Riemann, 42, 43

Delta-sum

Riemann, 42

Derivatives

distributional, 53, 75

Equiregulatedness, 17

Fell

topology, 126
Forward jump operator, 34

Function

bounded, 43, 47

bounded variation, 10, 28, 32

constant, 43

continuous, 39, 47

Darboux delta-integrable, 43

delta-differentiable, 37, 39, 47

delta-integrable, 42,47

gauge, 24

graininess, 35

Hilger-differentiable, 37

Kurzweil integrable, 24

Kurzweil-Henstock delta-integrable, 49

left-continuous, 68, 75

monotone, 47

nondecreasing, 15, 17, 30, 135

rd-continuous, 41, 44, 69, 72

regulated, $9,15,16,28,29,41,47,55$, 68,76

Riemann delta-integrable, 43

right-continuous, 68

step, 10, 28

Functional differential equations, 52

Functional dynamic equation on time scales, $67,72,91,98,105,114$

gauge, 62 
Generalized ordinary differential equation, Non-periodic averaging, 132, 141, 143, 145, 30, 31, 54, 59, 62, 91, 97, 105, 109, 113

Gronwall inequality, 29, 142, 162

Hausdorff

metric, 122, 123

topology, 123, 126

Hilger derivative, 37

Impulsive dynamic equation on time scales, 85

Impulsive functional dynamic equation on time scales, 82, 86, 87, 91, 105, 118, $122,123,128$

Impulsive measure FDE, 75, 77, 82, 86, 87, $91,100,105,116$

Integral

indefinite, 27

Kurzweil, 23, 24

Kurzweil-Henstock, 24, 85, 87

Kurzweil-Henstock-Stieltjes, 24, 69, 76, $82,84,135$

Integrally

asymptotically stable, 176, 190, 195, 196, 202

attracting, 176, 190, 194, 196, 202

stable, 176, 190, 194-196, 202

Lemma

Cousin, 25

Cousin for delta-gauge, 48

Saks-Henstock, 25

Lower Darboux delta-sum, 41

Lyapunov estable, 174

Measure differential equation, 24

Measure functional differential equation, 53, Stability theorem, 181, 185, 191, 196, 201

59, 63, 67, 77, 91, 105, 124, $127 \quad$ System, 23

Point

dense, 34

isolated, 34

left-dense, 34, 69

left-scattered, 34, 69

right-dense, 34, 39, 47, 69

right-scattered, 34, 39, 69

Prolongation property, 56, 59, 62, 95, 115

Regularly

asymptotically stable, 180, 195, 202

asymptotically stable with respect to perturbations, 181

attracting, 180, 194, 202

attracting with respect to perturbations, 180

stable, 179, 194, 202

stable with respect to perturbations, 180

Sequence

equiregulated, 21

Set

equicontinuous, 16

equiregulated, 10-12, 14, 16, 17, 20

relatively compact, 14, 16, 17

Solution

generalized ordinary differential equation, 30,32 
Tagged interval, 24

Tagged partition, 48

Theorem

Arzelá-Ascoli, 14, 16

Arzelá-Ascoli for Banach space, 17

Calculus Fundamental, Part I, 47

Calculus Fundamental, Part II, 47

Continuous dependence involving variable time scales, 122, 128

Continuous dependence on parameters, 105, 108, 112, 114, 116, 118

Existence and Uniqueness, 91, 95, 98, 100, 102

Hake-type, 26

Moore-Osgood, 20, 113

Time scale interval, 72, 86, 88, 102, 114, 118

Time scales, 33, 34

Uniform

convergence, 114, 121-124, 126, 127

uniformly asymptotically stable, 175

uniformly stable, 175

Upper Darboux delta-sum, 41

Variable time scales, 122 